\title{
Generalized mutual information metrics for affine brain image registration: proposal and evaluation
} Métricas de informação mútua generalizada para registro affine de imagens cerebrais: proposta e avaliação

Vinicius Pavanelli Vianna

Tese apresentada à Faculdade de Filosofia, Ciências e Letras de Ribeirão Preto da USP, como parte das exigências para a obtenção do título de Doutor em Ciências, obtido no Programa de Pós-Graduação em Física Aplicada à Medicina e Biologia.

RIBEIRÃO PRETO - SP 



\section{Generalized mutual information metrics for affine brain image registration: proposal and evaluation}

\section{Métricas de informação mútua generalizada para registro affine de imagens cerebrais: proposta e avaliação}

\section{Vinicius Pavanelli Vianna}

Tese apresentada à Faculdade de Filosofia, Ciências e Letras de Ribeirão Preto da USP, como parte das exigências para a obtenção do título de Doutor em Ciências, obtido no Programa de Pós-Graduação em Física Aplicada à Medicina e Biologia.

Orientador: Prof. Dr. Luiz Otavio Murta Junior 
Vinicius Pavanelli Vianna

Generalized mutual information metrics for affine brain image registration: proposal and evaluation

Métricas de informação mútua generalizada para registro affine de imagens cerebrais: proposta e avaliação / Vinicius Pavanelli Vianna. - RIBEIRÃO PRETO - SP, 2021-

165p. : il. (algumas color.) ; $30 \mathrm{~cm}$.

Supervisor: Prof. Dr. Luiz Otavio Murta Junior

Tese (Doutorado) - Versão Corrigida - UNIVERSIDADE DE SÃO PAULO

FACULDADE DE FILOSOFIA, CIÊNCIAS E LETRAS DE RIBEIRÃO PRETO

PROGRAMA DE PÓS-GRADUAÇÃO EM FÍSICA APLICADA À MEDICINA

E BIOLOGIA, 2021.

1. Informação Mútua. 2. Corregistro de Imagens Médicas. 2. Entropia. I. Orientador. II. Universidade de São Paulo. III. Faculdade de Filosofia, Ciências e Letras de Ribeirão Preto. IV. Título 


\title{
Generalized mutual information metrics for affine brain image registration: proposal and evaluation \\ Métricas de informação mútua generalizada para registro affine de imagens cerebrais: proposta e avaliação
}

\begin{abstract}
Tese apresentada à Faculdade de Filosofia, Ciências e Letras de Ribeirão Preto da USP, como parte das exigências para a obtenção do título de Doutor em Ciências, obtido no Programa de Pós-Graduação em Física Aplicada à Medicina e Biologia.
\end{abstract}

Trabalho aprovado. RIBEIRÃO PRETO - SP, 26 de outubro de 2021:

Prof. Dr. Luiz Otavio Murta Junior

Orientador

Prof. Dr. Constantino Tsallis

Doutor e Pesquisador do Departamento de

Física Teórica do Centro Brasileiro de

Pesquisas Físicas

Profa. Dra. Renata Ferranti Leoni

Professora Doutora do Departamento de

Física da Faculdade de Filosofia, Ciências e Letras de Ribeirão Preto - USP

Prof. Dr. Sérgio Shiguemi Furuie

Professor Titular do Departamento de Engenharia de Telecomunicações e Controle da Escola Politécnica - USP

RIBEIRÃO PRETO - SP 

Dedicated to my mother and the strong women of her family: Sheila, Maria Helena and Ignez Pavanelli. 



\section{Acknowledgements}

I would like to thank my supervisor, Dr. Luiz Otavio Murta Jr., for his guidance and wisdom through our research, and for introducing all the notions that made this thesis possible as the nonextensitivity, fractal dimensions, and generalized entropies. Dr. Murta's resilience made the research of Tsallis in medical images registration possible.

Thanks to the FAMB, DCM, and the department of physics at University of São Paulo, Ribeirão Preto, for the support and classes that allowed my academic growth, and all the staff that with their support help us students.

Thanks to Mr. Janderson Bispo Moreira, who had the patience to taught a 13 years old adolescent the art of programming. 



\section{Abstract}

Background and Objective: Image registration is a key operation in medical image processing, allowing a plethora of applications. Mutual information (MI) is consolidated as a robust similarity metric often used for medical image registration. Although MI provides a robust registration, it usually fails when the transform needed to register an image is too big due to MI local maxima traps.

Methods: In this paper, we propose and evaluate the Generalized MI (GMI), using Tsallis entropy as an affine registration metric with additive and non-additive equations. We assessed the metrics for separable affine transforms and exhaustively evaluated the GMI output signal seeking the maximum registration range through simulated gradient descent and a Monte Carlo simulation of real registrations with translated images.

Results: GMI metrics showed smoother isosurfaces that better lead the algorithm to the solution. Results show significantly prolonged registration ranges, without local maxima in the metric space, evaluated through a range of $150 \mathrm{~mm}$ for translations, $360^{\circ}$ for rotations, $[0.5,2]$ for scaling, and $[-1,1]$ for skewness, with a success rate of $99.99 \%, 88.20 \%, 99.99 \%$, and $99.99 \%$, respectively, in simulated gradient descent. We also obtained $99.75 \%$ success in the Monte Carlo simulation of 2,000 translation registrations with 1,113 double randomized subjects, using T1 and T2 brain magnetic resonance imaging (MRI).

Conclusions: The findings argue toward the reliability of GMI for long-range registrations. MI registration with Tsallis entropy requires specific entropic indexes $(q)$ per transformation parameter and also a proper selection of additive and non-additive equation depending on the transformation type. Also, with parallel computing and more computational power, a better analysis of the signal present on images, without simplifications like voxel sampling or histogram binning, provides a more efficient and robust MI registration, as the Monte Carlo simulation shows.

Keywords: Mutual Information, Tsallis Entropy, Medical imaging, Image registration, Brain images 



\section{Resumo}

Teoria e objetivo: Registro de imagens é uma operação fundamental no processamento de imagens médicas, permitindo uma variedade de aplicações. Informação Mútua (em inglês, Mutual Information - MI) é consolidada como uma métrica de similaridade robusta muito utilizada no registro de imagens médicas. Embora a MI forneça um registro robusto, ela usualmente falha quando a transformação necessária para registrar uma imagem é muito grande, por causa de armadilhas causadas por máximos locais.

Métodos: Nesta tese, eu proponho e avalio a Informação Mútua Generalizada (em inglês, Generalized MI - GMI), usando a entropia de Tsallis, como uma métrica de registro affine com equações aditivas e não-aditivas. Avaliamos as metricas em transformações affine separadamente e avaliamos exaustivamente o sinal gerado pela GMI procurando pela maior faixa de captura através de um gradiente descendente simulado e de uma simulação de registros reais de imagens transladadas usando Monte Carlo.

Resultados: As métricas GMI mostraram isosuperfícies mais suaves que guiam de forma melhor o algoritmo de registro para a solução. Os resultados mostraram faixas de registros significantemente prolongadas, sem máximos locais no espaço das métricas, avaliadas em uma faixa de $150 \mathrm{~mm}$ para as translações, $360^{\circ}$ para as rotações, [ 0.5, 2 ] para a escala e $[-1,1]$ para o cisalhamento, com uma taxa de sucesso de 99,99\%, 88,20\%, 99,99\%, e $99,99 \%$, respectivamente, no gradiente descendente simulado. Também obtive 99,75\% de sucesso na simulação de Monte Carlo de 2.000 registros de translações com 1.113 pacientes, duplamente randomizados, usando imagens cerebrais por ressonância magnética T1 e T2.

Conclusões: Os achados apontam para uma confiabilidade da GMI para registro de longoalcance. Registros usando MI com a entropia de Tsallis requerem índices entrópicos $(q)$ específicos para cada parâmetro da transformação, e também uma seleção adequada das equações aditivas e não-aditivas, dependendo do tipo de transformação sendo feito. Também, com a computação paralela e um maior poder computacional, uma análise melhor do sinal presente nas imagens, sem simplificações como amostragem de voxels ou discretização (binning) do histograma, provêem um registro com MI mais eficiente e robusto, conforme a simulação de Monte Carlo mostrou.

Palavras-chave: Informação Mútua, Imagens Médicas, Registro Imagens, Imagens do Cérebro 



\section{List of Figures}

Figure 1 - View from the Window at Le Gras 1826 or 1827 . . . . . . . . . . . . . 29

Figure 2 - "Hand mit Ringen" . . . . . . . . . . . . . . . . . . . . . . 29

Figure 3 - Computed tomography images . . . . . . . . . . . . . . . . . . . 29

Figure 4 - Magnetic Resonance images . . . . . . . . . . . . . . . . . . 30

Figure 5 - Lenna . . . . . . . . . . . . . . . . . . . 31

Figure 6 - MRI Dual 3D view . . . . . . . . . . . . . . . 32

Figure 7 - Volume information from the 3D Slicer software . . . . . . . . . 33

Figure 8 - Decomposition of a probabilities branch . . . . . . . . . . . 35

Figure 9 - MRI registration example . . . . . . . . . . . . . . . . . 40

Figure 10 - Two examples of registration from the INPE website . . . . . . . . . . 41

Figure 11 - Image registration block diagram . . . . . . . . . . . . . . . . . 42

Figure 12 - Head fixation device . . . . . . . . . . . . . . . . . . 43

Figure 13 - Linear interpolation of a function . . . . . . . . . . . . . 51

Figure 14 - Bilinear interpolation of a function. . . . . . . . . . . . . . . 52

Figure 15 - Effects of geometric transformation and interpolation on an image . . . 56

Figure 16 - Fractal shape of the Lake Mead, USA . . . . . . . . . . . . . . . . . . 59

Figure 17 - Mandelbrot fractals . . . . . . . . . . . . . . . . . . . . 61

Figure 18 - Human brain bisected in the sagittal plane . . . . . . . . . . . . . 71

Figure 19 - Brain Fractal Dimensions in relation to age . . . . . . . . . . . . . . 72

Figure 20 - BrainWeb Slice number 100 . . . . . . . . . . . . . . . . . . 73

Figure 21 - Example of the ParaView filters . . . . . . . . . . . . . . 78

Figure 22 - MI results from 2017 with translations on the $x$ axis . . . . . . . . . 82

Figure 23 - MI results from 2017 with translations on $y$ axis . . . . . . . . . . . . 83

Figure 24 - MI vs. Translation on $x$ axis . . . . . . . . . . . . . . . . . . . 84

Figure 25 - MI vs. Translation on $y$ axis . . . . . . . . . . . . . . . . 85

Figure 26 - MI function changes with 2D translation of an 3D image . . . . . . 87

Figure 27 - MI function changes with 2D rotation of an 3D image . . . . . . . . . 89

Figure 28 - 3D analysis introduction . . . . . . . . . . . . . . . . 92

Figure 29 - Translation Metric's 3D isosurfaces . . . . . . . . . . . . . . 93

Figure 30 - 3D analysis Comparison between ITK Mattes and Shannon . . . . . . 94

Figure 31 - 3D analysis Comparison between Shannon and Tsallis $(q=1.5)$. . . 95

Figure 32 - 3D isosurfaces using rotation with versors/quaternions . . . . . . . 97

Figure 33 - 3D analysis Comparison between ITK Mattes and Shannon for rotation with degrees. . . . . . . . . . . . . . . . . . . . . . . 98

Figure $34-3 \mathrm{D}$ analysis Comparison between Shannon and Tsallis $(q=1.3,4$ bits binning). . . . . . . . . . . . . . . . . . . 99 
Figure 35 - 3D isosurfaces using degrees rotation with no histogram binning. . . . . 101

Figure 36 - 3D isosurfaces using degrees rotation with histogram binning. . . . . . 101

Figure 37 - 3D isosurfaces using scaling transformations. . . . . . . . . . . . 103

Figure 38 - 3D isosurfaces using skewness transformations. . . . . . . . . . . . 103

Figure 39 - Tsallis' translation registration capacity as functions of the entropic index $q \ldots \ldots \ldots \ldots$. . . . . . . . . . . . . . . . . . . . . . . . . . .

Figure 40 - Tsallis' rotation registration capacity as functions of the entropic index $q 107$

Figure 41 - Tsallis' scaling registration capacity as functions of the entropic index $q 108$

Figure 42 - Tsallis' skewness registration capacity as functions of the entropic index $q 109$

Figure 43 - Metrics heatmaps . . . . . . . . . . . . . . . . . . . . . . . . . 110

Figure 44 - Monte Carlo registration essays for Mattes . . . . . . . . . . . . . . . 115

Figure 45 - Monte Carlo registration essays for UNC MIDAS . . . . . . . . . . . . 116

Figure 46 - Monte Carlo registration essays for Randomized test . . . . . . . . . . 117

Figure 47 - Monte Carlo registration essays as parallel coordinates . . . . . . . . 118

Figure 48 - Metrics registration capacity with different histogram binning settings . 123

Figure 49 - Metrics registration capacity with different histogram binning settings and colorbar limited . . . . . . . . . . . . . . . . . . . 124

Figure 50 - Example of affine registration using the meta-metric . . . . . . . . . . 125

Figure 51 - Monte Carlo results for translation with same image as fixed and moving, using the HCP 172635_3T_T1w_MPR1.nii . . . . . . . . . . . . . 151

Figure 52 - Monte Carlo results for translation with same image as fixed and moving, using the NAMIC N2_T1.nrrd . . . . . . . . . . . . . . . . . 154

Figure 53 - Monte Carlo results for translation with same image as fixed and moving,

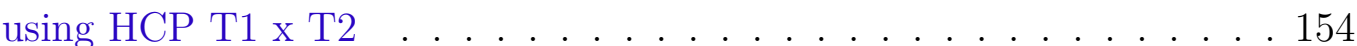

Figure 54 - Monte Carlo results for translation with same image as fixed and moving, only Mattes . . . . . . . . . . . . . . . . . . . . 155

Figure 55 - Monte Carlo results for translation with same image as fixed and moving, only Shannon . . . . . . . . . . . . . . . . . . . . . . 155

Figure 56 - Monte Carlo results for translation with same image as fixed and moving, only Tsallis . . . . . . . . . . . . . . . . . . . . 156

Figure 57 - Monte Carlo results stripplot for translation with different methods on the lines and the column loss is the final distance for the registration trials, $\log$ scale on y-axis . . . . . . . . . . . . . . . 157

Figure 58 - Monte Carlo results stripplot for translation with different methods on the lines and the column loss is the final distance for the registration trials, linear scale on y-axis

Figure 59 - Monte Carlo results stripplot combined with statistical point plot for translation with different methods on the lines and the column loss is the final distance for the registration trials, log scale on y-axis . . . . . 159 
Figure 60 - Monte Carlo results stripplot combined with statistical point plot for translation with different methods on the lines and the column loss is the final distance for the registration trials, linear scale on y-axis . . . 160 



\section{List of Tables}

Table 1 - Registration scenarios using Monte Carlo simulation. . . . . . . . . . . 79

Table 2 - Metrics registration percentage as function of the entropic index $q$, limited in the range $q \leq 1$. (A. means additive, N. means non-additive) . . . . . 111

Table 3 - Metrics registration percentage as function of the entropic index $q$, limited in the range $q \geq 1$. (A. mean additive, N. means non-additive) . . . . 112

Table 4 - Monte Carlo results, means and deviations, in mm. Acceptable distance percentage is the percentage of registrations that ended within this distance from the gold standard. . . . . . . . . . . . . . . . . . 114

Table 5 - Proposed meta-metric settings for affine registration. . . . . . . . . 123

Table 6 - Metrics registration percentage as function of the entropic index $q$, limited in the range $q \leq 1$, with 12 bits histogram binning. (A. means additive, N. means non-additive) . . . . . . . . . . . . . . . 136

Table 7 - Metrics registration percentage as function of the entropic index $q$, limited in the range $q \geq 1$, with 12 bits histogram binning. (A. means additive, N. means non-additive) . . . . . . . . . . . . . . . . 137

Table 8 - Metrics registration percentage as function of the entropic index $q$, limited in the range $q \leq 1$, with 10 bits histogram binning. (A. means additive, N. means non-additive) . . . . . . . . . . . . . . . . 138

Table 9 - Metrics registration percentage as function of the entropic index $q$, limited in the range $q \geq 1$, with 10 bits histogram binning. (A. means additive, N. means non-additive) . . . . . . . . . . . . . . .

Table 10 - Metrics registration percentage as function of the entropic index $q$, limited in the range $q \leq 1$, with 8 bits histogram binning. (A. means additive, N. means non-additive) . . . . . . . . . . . . . . . . 140

Table 11 - Metrics registration percentage as function of the entropic index $q$, limited in the range $q \geq 1$, with 8 bits histogram binning. (A. means additive, N. means non-additive) . . . . . . . . . . . . . . . . . . 141

Table 12 - Metrics registration percentage as function of the entropic index $q$, limited in the range $q \leq 1$, with 6 bits histogram binning. (A. means additive, N. means non-additive) . . . . . . . . . . . . . . . . . . . 142

Table 13 - Metrics registration percentage as function of the entropic index $q$, limited in the range $q \geq 1$, with 6 bits histogram binning. (A. means additive, N. means non-additive) . . . . . . . . . . . . . . . . 143

Table 14 - Metrics registration percentage as function of the entropic index $q$, limited in the range $q \leq 1$, with 4 bits histogram binning. (A. means additive, N. means non-additive) . . . . . . . . . . . . . . . . . . 144 
Table 15 - Metrics registration percentage as function of the entropic index $q$, limited in the range $q \geq 1$, with 4 bits histogram binning. (A. means additive, N. means non-additive) . . . . . . . . . . . . . . . . 145

Table 16 - Metrics registration percentage as function of the entropic index $q$, limited in the range $q \leq 1$, with 2 bits histogram binning. (A. means additive, N. means non-additive) . . . . . . . . . . . . . . 146

Table 17 - Metrics registration percentage as function of the entropic index $q$, limited in the range $q \geq 1$, with 2 bits histogram binning. (A. means additive, N. means non-additive) . . . . . . . . . . . . . . . . 147

Table 18 - Metrics registration percentage as function of the entropic index $q$, limited in the range $q \leq 1$, with 1 bit histogram binning. (A. means additive, $\mathrm{N}$.

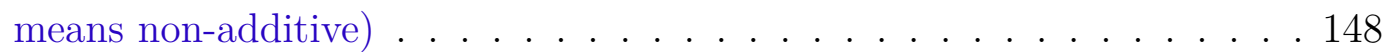

Table 19 - Metrics registration percentage as function of the entropic index $q$, limited in the range $q \leq 1$, with 1 bit histogram binning. (A. means additive, $\mathrm{N}$. means non-additive) . . . . . . . . . . . . . . . 149

Table 20 - Monte Carlo results for translation with same image as fixed and moving, using the HCP 172635_3T_T1w_MPR1.nii . . . . . . . . . . . . 152

Table 21 - Monte Carlo results for translation with same image as fixed and moving, using the NAMIC N2_T1.nrrd image . . . . . . . . . . . . . . 153

Table 22 - Monte Carlo results for translation with same image as fixed and moving,

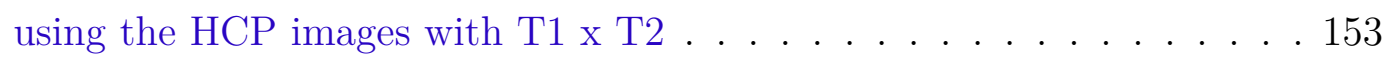

Table 23 - All Monte Carlo results collected . . . . . . . . . . . . . . . . . . 161 


\section{List of abbreviations and acronyms}

\begin{tabular}{|c|c|}
\hline $1 \mathrm{D}$ & One-dimensional \\
\hline $2 \mathrm{D}$ & Two-dimensional \\
\hline $3 \mathrm{D}$ & Three-dimensional \\
\hline $\mathrm{BCE}$ & Before Common Era, i.e., before Christ \\
\hline BG & Boltzmann-Gibbs \\
\hline BrainWeb & BrainWeb: Simulated Brain Database \\
\hline $\mathrm{CT}$ & Computed Tomography \\
\hline DXA & Dual energy X-ray absorptiometry, previously DEXA \\
\hline EMI & Enhanced mutual information \\
\hline EMMA & $\begin{array}{l}\text { Empirical entropy manipulation and analysis, from (VIOLA; WELLS, } \\
\text { 1997) }\end{array}$ \\
\hline GMI & Generalized mutual information \\
\hline $\mathrm{HCP}$ & Human Connectome Project \\
\hline INPE & $\begin{array}{l}\text { National Institute for Space Research in Brazil (Instituto Nacional de } \\
\text { Pesquisas Espaciais) }\end{array}$ \\
\hline ITK & Insight toolkit \\
\hline MI & Mutual information \\
\hline MRI & Magnetic resonance imaging \\
\hline NAMIC & National Association of Mutual Insurance Companies \\
\hline NMI & Normalized mutual information \\
\hline $\mathrm{PCA}$ & Principal component analysis \\
\hline PET & Position emission tomography \\
\hline $\mathrm{PNG}$ & Portable network graphic \\
\hline $\mathrm{RF}$ & Radio frequency \\
\hline
\end{tabular}


RGBA Red, Green, Blue, Alpha, i.e., a color pixel format

RGB Red, Green, Blue, i.e., a color pixel format

RMI Regional mutual information

ROI Region of interest

SEMI Spatially encoded mutual information

SPECT Single photon emission computed tomography

TRE Target registration errors

Tsallis/Rényi A. Tsallis/Rényi additive equation

Tsallis/Rényi N. Tsallis/Rényi non-additive equation

UNC The University of North Carolina at Chapel Hill

VTI Visualization toolkit Image

VTK Visualization Toolkit 


\section{List of symbols}

\begin{tabular}{|c|c|}
\hline$D$ & fractal dimension \\
\hline$F_{m}(\cdot)$ & metric function \\
\hline$H$ & Shannon entropy \\
\hline$H(\cdot, \cdot)$ & Shannon joint entropy \\
\hline$H(\cdot \mid \cdot)$ & Shannon conditional entropy \\
\hline$H_{\alpha}(\cdot)$ & Rényi entropy function \\
\hline$I(\cdot)$ & mutual information \\
\hline$K$ & Shannon entropy constant, normally $K=-1$ \\
\hline$R$ & rate of transmission \\
\hline$R_{x}(\gamma)$ & rotation matrix for $x$ axis, i.e., pitch \\
\hline$R_{y}(\beta)$ & rotation matrix for $y$ axis, i.e., yaw \\
\hline$R_{z}(\alpha)$ & rotation matrix for $z$ axis, i.e., roll \\
\hline$S_{q}(\cdot), H_{q}(\cdot)$ & Tsallis entropy function \\
\hline$S_{x}$ & scaling parameter of $x$ axis \\
\hline$S_{y}$ & scaling parameter of $y$ axis \\
\hline$S_{z}$ & scaling parameter of $z$ axis \\
\hline$T$ & set of transformation parameters \\
\hline$T(\cdot)$ & geometric transformation function \\
\hline$\hat{T}$ & final transformation parameters, i.e., solution parameters \\
\hline$\phi$ & rotation angle \\
\hline$\psi$ & rotation angle \\
\hline$\theta$ & rotation angle \\
\hline$\hat{\mathbf{i}}$ & unity vector for $x$ axis \\
\hline
\end{tabular}




$\begin{array}{ll}\hat{\mathbf{j}} & \text { unity vector for } y \text { axis } \\ \hat{\mathbf{k}} & \text { unity vector for } z \text { axis } \\ k_{x y} & \text { skewness parameters of } x \text { and } y \text { axes } \\ k_{x z} & \text { skewness parameters of } x \text { and } z \text { axes } \\ k_{y x} & \text { skewness parameters of } y \text { and } x \text { axes } \\ k_{y z} & \text { skewness parameters of } y \text { and } z \text { axes } \\ k_{z y} & \text { skewness parameters of } z \text { and } y \text { axes } \\ k_{z z} & \text { skewness parameters of } z \text { axis } \\ p_{i} & \text { probability of event } i \text { occurence } \\ q & \text { Tsallis entropic index } \\ t_{x} & \text { transformation parameters of } x \text { axis } \\ t_{y} & \text { transformation parameters of } y \text { axis } \\ t_{z} & \text { transformation parameters of } z \text { axis } \\ u(\cdot) & \text { fixed image function } \\ & \end{array}$




\section{Contents}

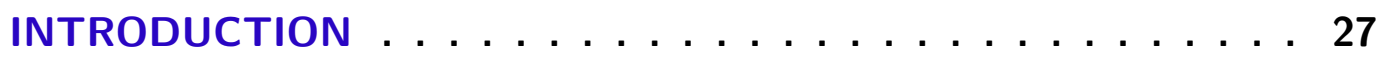

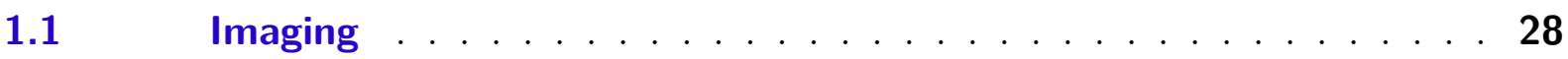

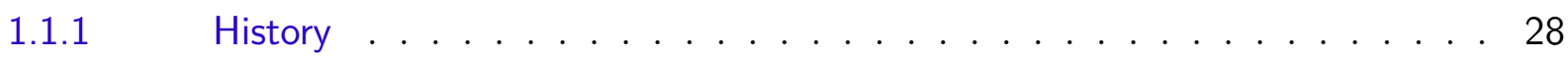

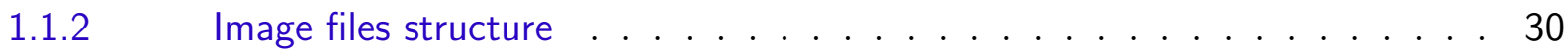

1.2 Shannon - A Mathematical Theory of Communication . . . . . . . 34

1.2.1 The $H$ measurement . . . . . . . . . . . . . . . . . . . . 34

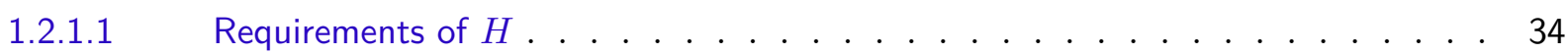

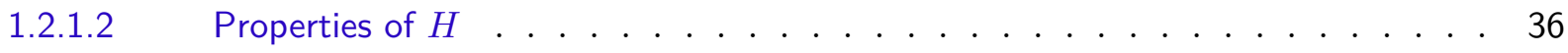

1.2.2 Practical equations on Shannon Entropy . . . . . . . . . . . . . 37

$1.2 .3 \quad$ Mutual Information . . . . . . . . . . . . . . . . . . 38

$1.3 \quad$ Image Registration . . . . . . . . . . . . . . . . 40

1.3.1 Registration Algorithm . . . . . . . . . . . . . . . . 41

1.3.2 History of Medical Image Registration . . . . . . . . . . . . 42

$1.4 \quad$ Geometric Transformation . . . . . . . . . . . . . . . . . 45

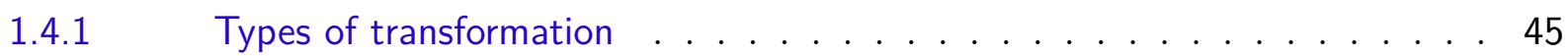

1.4 .2 Translation . . . . . . . . . . . . . . . 46

1.4.3 Matrix Transformation . . . . . . . . . . . . . . . 46

1.4.4 Rotation Matrices . . . . . . . . . . . . . . . . . . 46

1.4.5 Rotation using roll, yaw, and pitch . . . . . . . . . . . . 48

$1.4 .6 \quad$ Scaling Matrix . . . . . . . . . . . . . . . . . . . . . . 48

1.4 .7 Skewness Matrix . . . . . . . . . . . . . . . . . . . . . . . . 49

$1.4 .8 \quad$ Affine Matrix . . . . . . . . . . . . . . . . . . 49

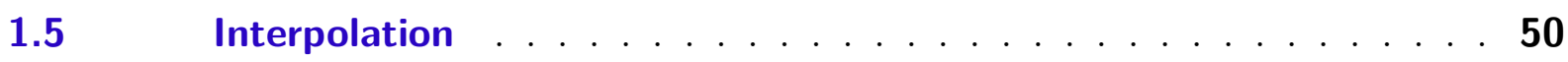

$1.5 .1 \quad$ Nearest Neighbor . . . . . . . . . . . . . . . . . . . 50

1.5 .2 Linear interpolation . . . . . . . . . . . . . . . 50

1.5.2.1 Bilinear Interpolation . . . . . . . . . . . . . . . . 52

$1.6 \quad$ Image Registration with Mutual Information . . . . . . . . 54

1.6.1 Shannon Entropy Calculations for Images . . . . . . . . . . . . 54

1.6.2 Mutual Information for Registration . . . . . . . . . . . . 54

1.6.2.1 Mathematical Analysis . . . . . . . . . . . . . . . . 54

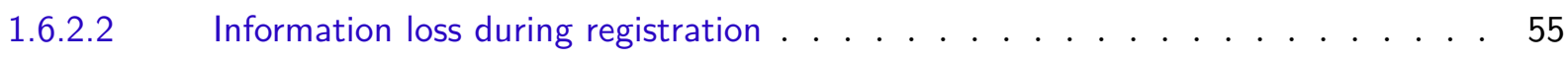

$1.7 \quad$ Classical $\mathrm{Ml}$ registration papers $\ldots \ldots \ldots \ldots$

1.7.1 Ml derived works in literature . . . . . . . . . . . . 57

$1.8 \quad$ Fractal Dimensions . . . . . . . . . . . . . . . . 59

$1.9 \quad$ Generalized Entropies . . . . . . . . . . . . . 63 
$1.9 .1 \quad$ Tsallis . . . . . . . . . . . . . . . . . . 63

1.9.1.1 Tsallis q-logarithm and q-exponential . . . . . . . . . . . . . . . 64

1.9.1.2 Tsallis Mutual Information . . . . . . . . . . . . . . . . 65

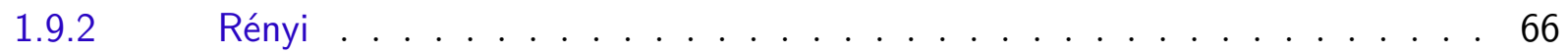

1.10 Nonextensivity . . . . . . . . . . . . . . . . . 66

$1.10 .1 \quad$ Physical properties . . . . . . . . . . . . . . . 66

$1.10 .2 \quad$ Nonextensitivy . . . . . . . . . . . . . . . 66

$1.10 .3 \quad$ Non-Additivity . . . . . . . . . . . . . . . . . 67

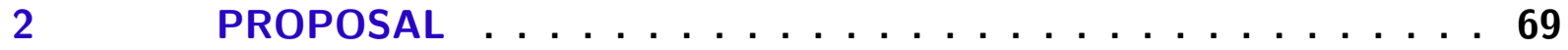

$2.1 \quad$ Hypothesis . . . . . . . . . . . . . . . . 69

2.2 Rationale . . . . . . . . . . . . . . . . . . . 69

2.2.1 Brain Fractal Geometry . . . . . . . . . . . . . . . . . . . . 69

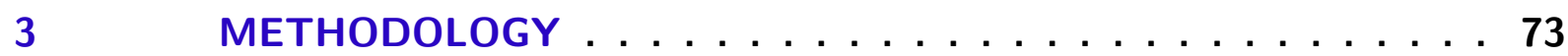

3.12 2D images analysis using 1D transformations . . . . . . . . 73

$3.2 \quad 3 \mathrm{D}$ images analysis using 2D transformations . . . . . . . . . 74

3.3 3D images analysis using 3D transformations . . . . . . . . 75

3.3.1 Metric image visualization . . . . . . . . . . . . . . . . 75

3.3.2 ParaView Analysis . . . . . . . . . . . . . . . . . . . 76

3.3.3 Naive registration capacity . . . . . . . . . . . . . . . . . 77

$3.4 \quad$ Monte Carlo Simulation . . . . . . . . . . . . . . . . . . 79

$4 \quad$ RESULTS AND DISCUSSION . . . . . . . . . . . . 81

$4.12 \mathrm{D}$ images with 1D analysis (2017) . . . . . . . . . 81

$4.2 \quad 3 \mathrm{D}$ analysis with $2 \mathrm{D}$ translations and rotations ........ 86

$4.2 .1 \quad$ Translation . . . . . . . . . . . . . . . . . . 86

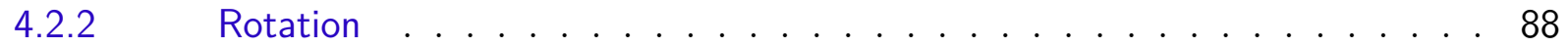

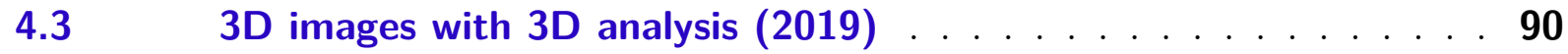

$4.3 .1 \quad$ Introduction . . . . . . . . . . . . . . . . . . 90

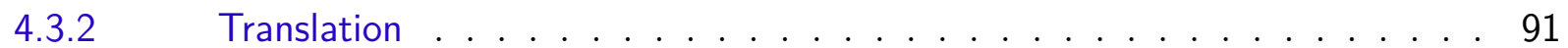

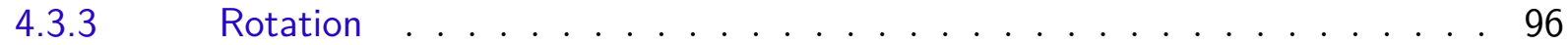

4.3.3.1 Versors . . . . . . . . . . . . . . . . . . 96

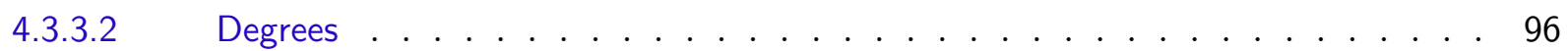

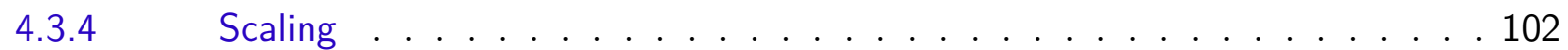

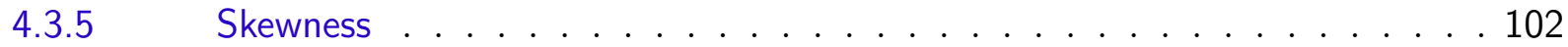

$4.4 \quad$ Registration capacity . . . . . . . . . . . . . . . . 104

$4.5 \quad$ Monte Carlo Results . . . . . . . . . . . . . . . . . . . . . . . 113

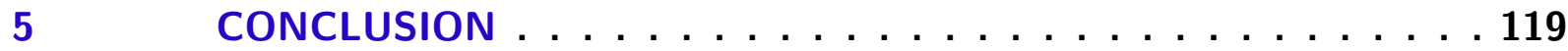

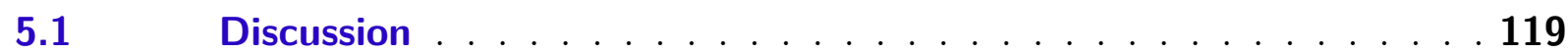




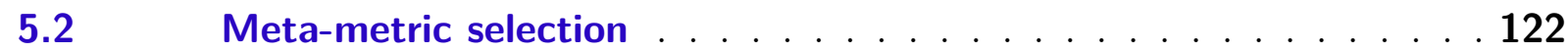

5.2.1 Affine registration example . . . . . . . . . . . . . . . . 124

$5.3 \quad$ Conclusion . . . . . . . . . . . . . . . . . . 124

$5.3 .1 \quad$ Future work . . . . . . . . . . . . . . . 125

BIBLIOGRAPHY .................... 127

APPENDIX A - FULL DATA COLLECTED . . . . . . . . . . . 135

A.1 Full data collected from 3D images and 3D transformations . . . 135

APPENDIX B - MONTE CARLO . . . . . . . . . . . . . 151

B.1 Monte Carlo Results . . . . . . . . . . . . . . . . . . . . 151

B.1.1 Same image - High Quality . . . . . . . . . . . . . . . 151

B.1.2 Same image - Low Quality . . . . . . . . . . . . . . . . . . . 152

B.1.3 Same patient - T1 x T2 - High Resolution . . . . . . . . . . 152

B.1.4 Monte Carlo Randomized . . . . . . . . . . . . . . . . . 153 



\section{Introduction}

The question I formulate in this thesis is:

\section{"Can the use of generalized entropies on mutual information metrics improve the affine registration of brain images?"}

The core of this question is generalized entropy. How different is the generalized entropy perception of the physical space to the classical Boltzmann-Gibbs-Shannon entropy, designated by just Shannon in this thesis? This thesis's physical space is also constrained by the mutual information of medical brain T1 and T2 magnetic resonance imaging (MRI).

The introduction (chapter 1 ) is organized to provide an understanding of the processes involved in the image registration, beginning with the imaging (sec. 1.1), a process which the physical entity, e.g., a human head, is converted into an image, which contains information about the original physical properties. This information is normally analyzed using Shannon entropy (sec. 1.2) to drive an image registration algorithm (sec. 1.3) which will transform (sec. 1.4) and interpolate (sec. 1.5) the moving image as good as possible to match a fixed image, in order to register both images.

The most common registration algorithm used in medicine is mutual information (sec. 1.6), with vast literature about its performance and how to improve it (sec. 1.7).

This thesis proposes to improve the image registration by replacing the Shannon entropy with a generalized entropy (sec. 1.9). The difference between generalized and Shannon entropy, besides the math, is that generalized entropies are non-extensive (sec. 1.10). They consider that entropy also depends on long-range interaction in the physical system and not only on basic modeling as mass or volume.

Also, the mathematics of generalized entropies and nonextensive systems connect to fractal dimensions (sec. 1.8). As our brain possesses a fractal geometry (sec. 2.2.1), generalized entropies should be best suited to register brain images.

Inspiration is taken from Lord Kelvin's famous quote: "If you cannot measure it, you cannot improve it.", to answer the question proposed (chapter 2).

Measurement of our metrics in several planned experiments (chapter 3) provides results (chapter 4) on how the generalized metric changes our metrics.

Finally, I discuss the results to conclude (chapter 5) the best settings and ensemble of metrics to register brain images based on our measurements. 


\subsection{Imaging}

Imaging is the transformation of information between domains, e.g., physical properties from a scene are transformed to visual information, e.g., a gray level representing bone density.

\subsubsection{History}

Photography is the process of drawing with light, with the word being the junction of the Greek words phōto's and graphé. Its first appearances in human history, in the form of Camera Obscura, may occur in prehistory in Neolithic structures. However, a written record first appears in the Chinese text Mozi, dated to the 4th century before common era (BCE) (CAMERA..., 2021).

As humans evolved, Schulze discovered, in experiments published in 1719, (BIBLIOTHECAE..., 1718) that silver nitrate reacts with light and could draw letters on bottles using stencils, but ephemeral as further exposure to light, or simply shaking the bottle, would remove the effect. However, it was only in the mid-1820s that Joseph Nicéphore Niépce succeeded in non-ephemeral imaging using a technique called heliography (NICéPHORE..., 2021). It is also from Niépce, the oldest surviving photography of a real-world scene (Fig. 1).

Imaging techniques for medical purposes started with Wilhelm Conrad Röntgen's discovery of x-rays in 1895 (Fig. 2), creating the science field now called Radiography, using the prefix from the Latin word Radius, meaning a ray, and the Greek word graphé. Radiography evolved to different techniques, where the method used by Röntgen is related to what is now called Projectional Radiography, or just plain Radiography. More advanced techniques were created from Radiography, like Computed Tomography (CT) (CORMACK, 1963; HOUNSFIELD, 2014), capable of 3D imaging (Fig. 3), and Dual-energy X-ray absorptiometry (DXA) (WAHNER et al., 1988), capable of bone densitometry and used primarily for osteoporosis tests.

Magnetic Resonance Imaging was created by (LAUTERBUR, 1973) and further improved by (MANSFIELD; GRANNELL, 1975), both share the Nobel Prize in Physiology or Medicine 2003 due to this discovery. The technique uses magnetic fields and radiofrequency $(\mathrm{RF})$ signals to polarize the spin of specific atomic nuclei. This polarization produces another RF signal that can be detected and analyzed to produce an image of the atomic nuclei distribution (HOULT; BHAKAR, 1997). An example of an MRI can be seen in Fig. 4.

Other techniques used in medical imaging consist of ultrasound, endoscopy, elastography, tactile imaging, thermography, medical photography, nuclear medicine functional imaging techniques as positron emission tomography (PET) and single-photon emission 
Figure 1 - View from the Window at Le Gras 1826 or 1827

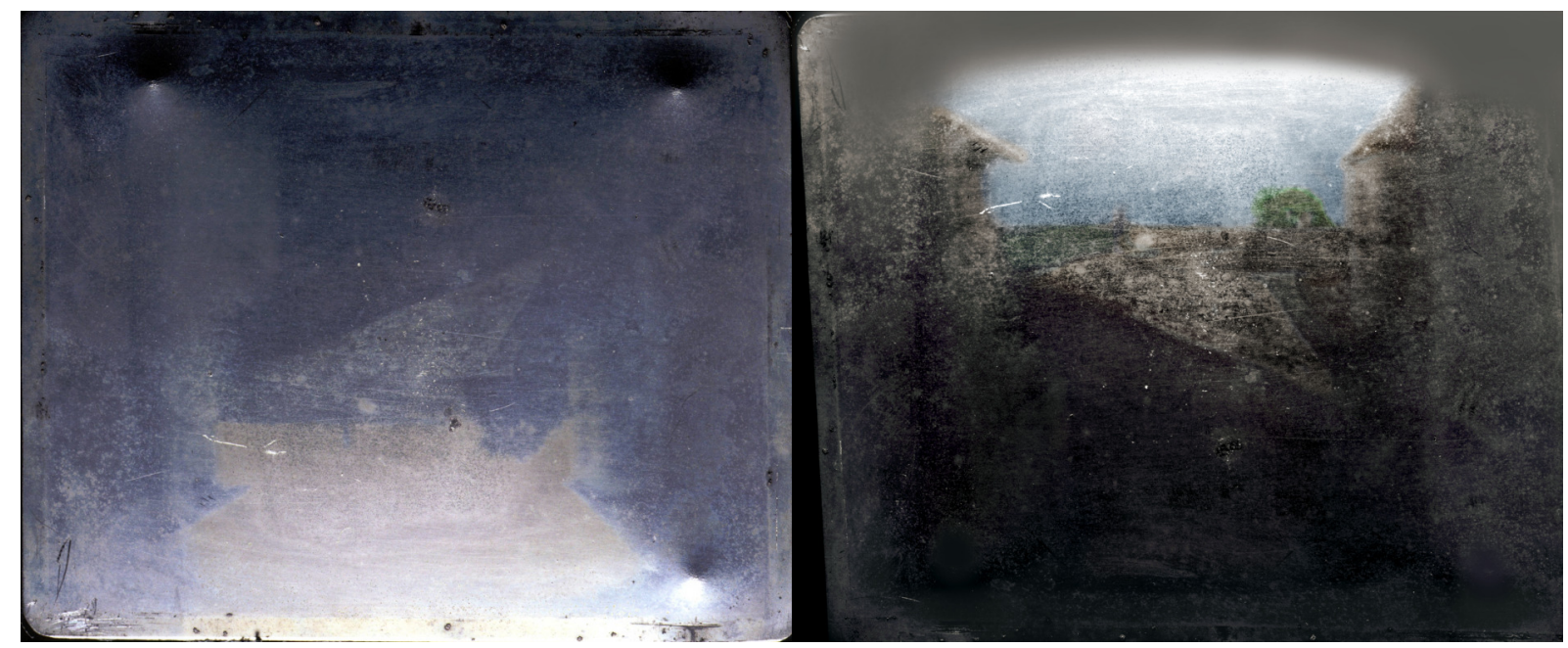

This is the oldest surviving photograph; Original plate (left), colorized reoriented enhancement (right). Source: Jonnychiwa - Own work, CC BY-SA 4.0, <https://commons.wikimedia.org/w/ index.php?curid $=88887615>$.

Figure 2 - "Hand mit Ringen"

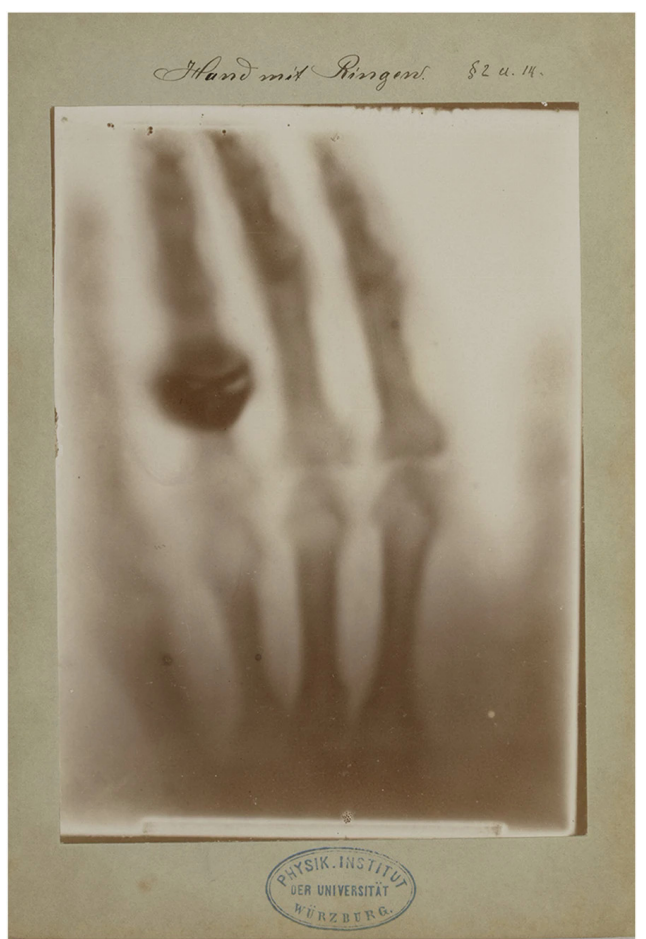

The first radiography, taken by Wilhelm Conrad Röntgen on December 22nd, 1895, showing the hand of his wife, Mrs. Anna Bertha Ludwig. Source: (ZONNEVELD, 2020).
Figure 3 - Computed tomography images

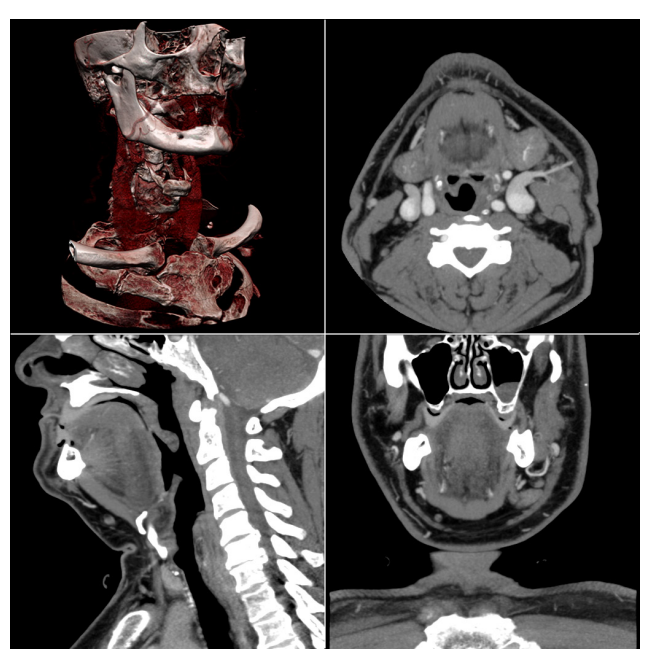

Images generated from computed tomography, including a 3D rendered image at the upper left. Source: en:User:ChumpusRex - CC BY-SA 3.0, <https:// commons. wikimedia. org/w/index.php?curid=6026473> . 
Figure 4 - Magnetic Resonance images

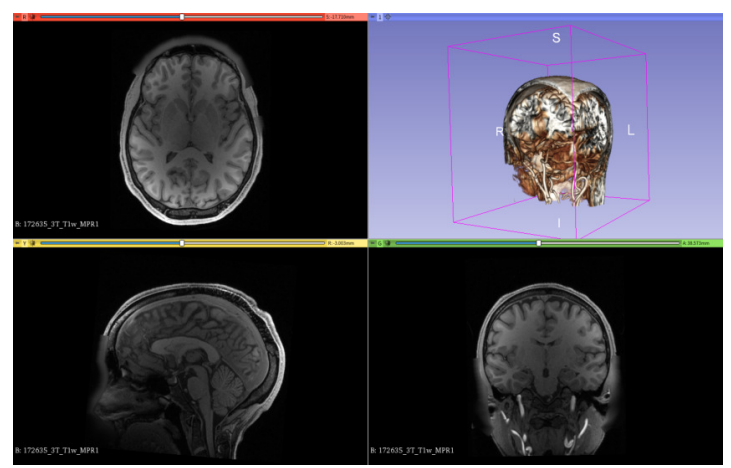

Images generated from Magnetic Resonance using the 3D Slicer software, using data from the Human Connectome Project (HCP) Dataset. Source: author.

computed tomography (SPECT). (MEDICAL..., 2017)

The central aspect of Imaging, and Medical Imaging, that are important to the present work is that those techniques provide ways to transform physical properties into information, which will be analyzed in the following sections. It is essential not to forget that all the information in an image, including a medical one, comes from physical property and brings physical relations present to the scene being imaged to the information stored in the image.

\subsubsection{Image files structure}

To be processed by a computer, images generated by the techniques presented need to be converted to the digital domain, e.g., a computer file. The conversion process is technique-dependent and can vary from simple digital scanning, very similar to the one used to scan a paper photograph, to more complex transformation, like the MRI conversion from the k-space, i.e., frequency components of a discrete Fourier space transform, to gray levels in a spatial grid.

In the digital domain, for our purposes, image data are structured in a bitmap, a collection of single elements called pixels, in the case of 2D images, or voxels, in the case of 3D images. Image formats for vectorial data are used to store geometric shapes, but those vectorial images are generally not used to store information generated by imaging techniques. Some annotations made on the images, like regions of interest (ROI), can be stored in a vectorial format attached to the original bitmap with instructions on aligning both formats.

Fig. 5 shows the classical "Lenna" image, used in the academy for image processing research, and also shows a zoom of the image containing Lenna's eye with a grid delimitating the pixels elements. In a typical image, one can see that we cannot distinguish one pixel from another since they are tiny, giving the illusion of a complete detailed image. The 
Figure 5 - Lenna
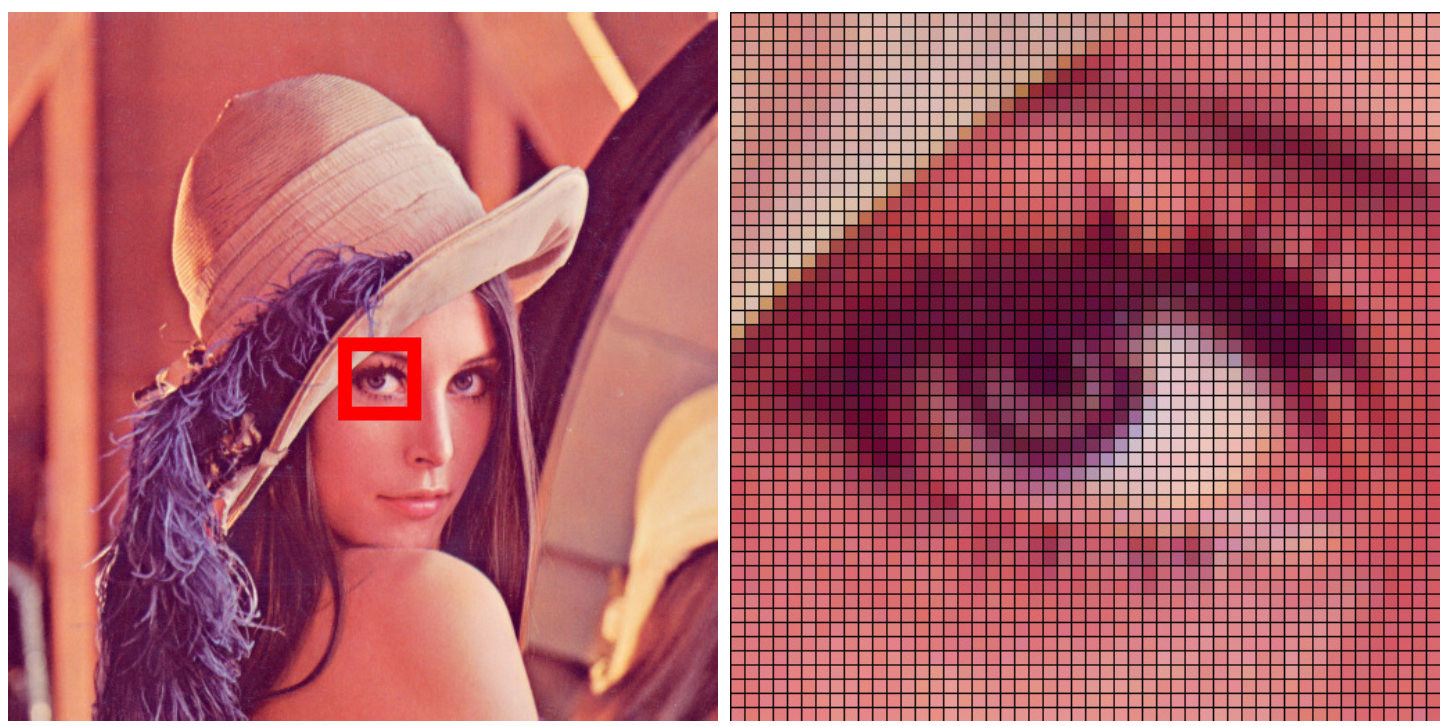

Classical image of Lenna overly used in the academy; By Original full portrait: "Playmate of the Month". Playboy Magazine. November 1972, photographed by Dwight Hooker. Source: USC-SIPI image database, Fair use, <https://en.wikipedia.org/w/index.php?curid=20658476>.

bitmap term comes from the effect shown in the (b) figure, where we can see the bitmap of multiple pixels, each with its color value, making the full image.

Depending on the image used, we can have a single channel for gray levels, or multiple channels for color images, i.e., RGB for red, green, and blue channels. Also, modern images allow a fourth channel called the "alpha" channel that controls the pixels' opacity, or transparency, in the bitmap.

When we look at the Lenna.png file, containing the image in Fig. 5, we can see something like this:

Lenna.png: PNG image data, 512 x 512, 8-bit/color RGBA, non-interlaced

The above command output means that we are analyzing the Lenna.png file. It is a PNG image data file containing $512 \times 512$ pixels in a bitmap with each pixel having 8 bits per color channel, with four channels: RGBA (Red, Green, Blue, and Alpha), and stored in a non-interlaced way.

The concept of a pixel extends, in a 3D image, to a voxel. A pixel consists of a rectangular region in the bitmap, while the voxel consists of a rectangular prism or cuboid. It can be seen as a simple extrusion adding depth to a pixel to form a 3D shape called a voxel. The 3D images can be seen as multiple stacked layers of 2D images.

Fig. 6 shows two 3D renders of one image from the HCP dataset, used in sec. 3.3, with the left view being under-sampled in a 3:1 ratio, the bottom slice views are also undersampled, showing the pixels from the images, the 3D render on the right uses cubic 
Figure 6 - MRI Dual 3D view

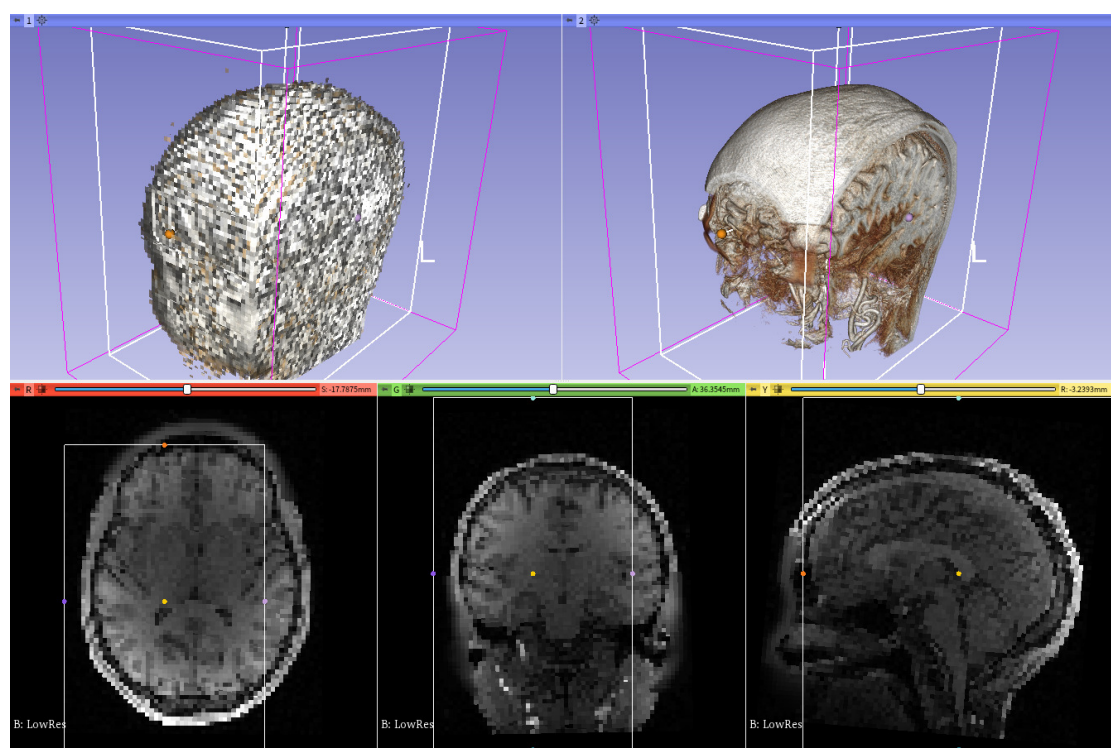

Dual 3D view, with the left one being under-sampled in a 3:1 ratio to better show the voxels and the right enriched with interpolation and rendered with material and opacity. Images generated from Magnetic Resonance using the 3D Slicer software, using data from the HCP Dataset. Source: author.

interpolation and was rendered with material and opacity options to see the inner details better.

The bitmaps presented can have different proportions, i.e., relations between the dimensions like width and height, forming a non-square but rectangular shape for the pixels. In medical images, we call this spacing, and it translates to the dimensions of a pixel or voxel in the image. When all dimensions are the same on an image, it will have a square pixel or a cuboid voxel. This image is called isotropic or isospaced. In some situations, like in MRI, we can have a greater resolution in width and height than the depth resolution.

Fig. 7 shows the detail of an image file from the HCP dataset, with dimensions of $256 \times 320 \times 320$ and spacing of $0.7 \times 0.7 \times 0.7(\mathrm{~mm})$, this means that we have 320 layers of $256 \times 3202 \mathrm{D}$ images, and we also have an isotropic image where the voxel is a cube with a size of $0.7 \mathrm{~mm}$. There are other details shown, like the Origin point of the image in the space and a direction matrix. Those details are mostly found only in medical images and provide details on how to align this image on a physical space, e.g., where our image starts and the direction that we have to construct our voxels to perfectly align the image on the physical space. 
Figure 7 - Volume information from the 3D Slicer software

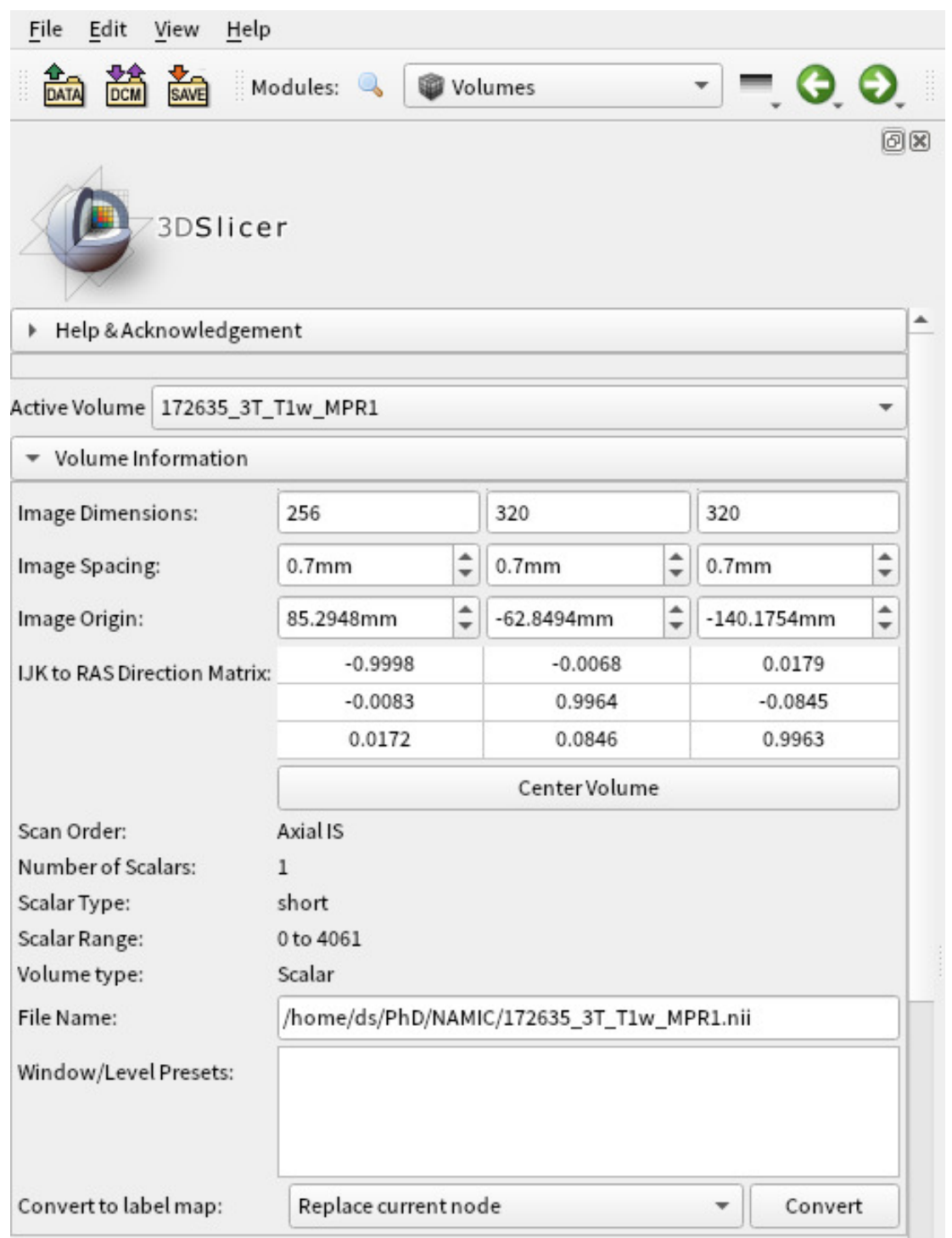

Volume information showing image dimensions and spacing details. Images generated from Magnetic Resonance using the 3D Slicer software, using data from the HCP Dataset. Source: author. 


\title{
1.2 Shannon - A Mathematical Theory of Communication
}

Claude Elwood Shannon wrote in 1948 the work "A Mathematical Theory of Communication" (SHANNON; WEAVER, 1963), giving birth to the field now called information theory and further allowed Communications and Information Technologies.

\begin{abstract}
According to an anonymous obituary in The Times newspaper on 12 March 2001, Claude Shannon was

[a] playful genius who invented the bit, separated the medium from the message, and laid the foundations for all digital communications.... [He] single-handedly laid down the general rules of modern information theory, creating the mathematical foundations for a technical revolution. Without his clarity of thought and sustained ability to work his way through intractable problems, such advances as e-mail and the World Wide Web would not have been possible.
\end{abstract}

The great breakthroughs in understanding which heralded the convergence of computing and communications. To colleagues in the corridors at the Massachusetts Institute of Technology who used to warn each other about the unsteady advance of Shannon on his unicycle, it may have seemed improbable that he could remain serious for long enough to do any important work. Yet the unicycle was characteristic of his quirky thought processes, and became the topsy-turvy symbol of unorthodox progress towards unexpected theoretical insights.

The ability to make astonishing leaps beyond the intellects of his colleagues (all the more remarkable at MIT, the forcing house of technological theory) had to be acknowledged as genius, and Claude Shannon was recognized as a giant throughout the industry.

(JAMES, 2009)

\subsubsection{The $H$ measurement}

In his book, Shannon start by defining, in his words, "a quantity which will measure, in some sense, how much information is "produced" by such a process, or better, at what rate information is produced". Furthermore, to formulate a function to measure this information, he defines:

Suppose we have a set of possible events whose probabilities of occurrence are $p_{1}, p_{2}, \ldots, p_{n}$. These probabilities are known but that is all we know concerning which event will occur. Can we find a measure of how much "choice" is involved in the selection of the event or of how uncertain we are of the outcome? If there is such a measure, say $H\left(p_{1}, p_{2}, \ldots, p_{n}\right)$, it is reasonable to require of it the following properties:

(SHANNON; WEAVER, 1963)

\subsubsection{Requirements of $H$}

The requirements on $H$ that Shannon defines are the following:

1. $H$ should be continuous in the $p_{i}$ space. 
Figure 8 - Decomposition of a probabilities branch

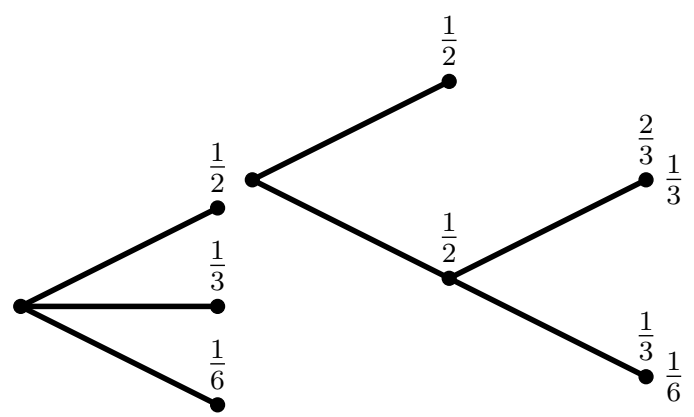

Decomposition of a probabilities branch should not change the entropy. Source: adapted from (SHANNON; WEAVER, 1963) by the author.

2. If all the $p_{i}$ are equal, $p_{i}=\frac{1}{n}$, then $H$ should be a monotonic increasing function of $n$. With equally likely events there is more choice, or uncertainty, when there are more possible events.

3. If a choice is broken down into two successive choices, the original $H$ should be the weighted sum of the individual values of $H$. The meaning of this is illustrated in fig. 8. At the left we have three possibilities $p_{1}=\frac{1}{2}, p_{2}=\frac{1}{3}, p_{3}=\frac{1}{6}$. On the right we first choose between two possibilities each with probability $\frac{1}{2}$, and if the second occurs make another choice with probabilities $\frac{2}{3}, \frac{1}{3}$. The final results have the same probabilities as before. We require, in this special case, that

$$
H\left(\frac{1}{2}, \frac{1}{3}, \frac{1}{6}\right)=H\left(\frac{1}{2}, \frac{1}{2}\right)+\frac{1}{2} H\left(\frac{2}{3}, \frac{1}{3}\right)
$$

The coefficient $\frac{1}{2}$ is because this second choice only occurs half the time.

The only $H$ that satisfies the three conditions imposed by Shannon is of the form:

$$
H=-K \sum_{i=1}^{n} p_{i} \log p_{i}
$$

where $\mathrm{K}$ is a positive constant.

Later, Shannon explain:

Quantities of the form $H=\sum p_{i} \log p_{i}$ (the constant $K$ merely amounts to a choice of a unit of measure) play a central role in information theory as measures of information, choice and uncertainty. The form of $H$ will be recognized as that of entropy as defined in certain formulations of statistical mechanics where $p_{i}$ is the probability of a system being in cell $i$ of its phase space. $H$ is then, for example, the $H$ in Boltzmann's famous $H$ theorem. We shall call $H=\sum p_{i} \log p_{i}$ the entropy of the set of probabilities $p_{1}, \ldots, p_{N}$ If $x$ is a chance variable we will write $H(x)$ for its entropy; thus $x$ is not an argument of a function but a label for a number, to differentiate it from $H(y)$ say, the entropy of the chance variable $y$.

(SHANNON; WEAVER, 1963, p. 11) 


\subsubsection{Properties of $H$}

The properties of the function $H$ are the following, as defined by (SHANNON; WEAVER, 1963):

1. $H=0$ if, and only if, all the $p_{i}$ but one are zero, this one having the value unity. Thus, only when we are certain of the outcome does $H$ vanish. Otherwise, this $H$ is positive.

2. For a given $n, H$ is a maximum and equal to $\log n$ when all the $p_{i}$ are equal (i.e., $\frac{1}{n}$ ). This is also intuitively the most uncertain situation.

3. Suppose there are two events, $x$ and $y$, in question with $m$ possibilities for the first and $n$ for the second. Let $p(i, j)$ be the probability of the joint occurrence of $i$ for the first and $j$ for the second. The entropy of the joint event is

$$
H(x, y)=-\sum_{i, j} p(i, j) \log p(i, j)
$$

while

$$
H(x)=-\sum_{i, j} p(i, j) \log \sum_{j} p(i, j) \quad H(y)=-\sum_{i, j} p(i, j) \log \sum_{i} p(i, j)
$$

It is easily shown that

$$
H(x, y) \leq H(x)+H(y)
$$

with equality only if the events are independent (i.e, $p(i, j)=p(i) p(j))$. The uncertainty of a joint event is less than or equal to the sum of the individual uncertainties.

4. Any change toward equalization of the probabilities $p_{1}, p_{2}, \ldots, p_{n}$ increases $H$. Thus, if $p_{1}<p_{2}$, and we increase $p_{1}$, decreasing $p_{2}$ an equal amount, so that $p_{1}$ and $p_{2}$ are more nearly equal, then $H$ increases. More generally, if we perform any "averaging" operation on the $p_{i}$ of the form

$$
p_{i}^{\prime}=\sum_{j} a_{i j} p_{j}
$$

where $\sum_{i} a_{i j}=\sum_{j} a_{i j}=1$, and all $a_{i j} \leq 0$, then $H$ increases (except in the special case where this transformation amounts to no more than a permutation of the $p_{j}$ with $H$ of course remaining the same).

5. Suppose there are two chance events $x$ and $y$ as in 3, not necessarily independent. For any particular value $i$ that $x$ can assume, there is a conditional probability $p_{i}(j)$ that $y$ has the value $j$. This is given by

$$
p_{i}(j)=\frac{p(i, j)}{\sum_{j} p(i, j)}
$$


We define the conditional entropy of $y, H_{x}(y)$ as the average of the entropy of $y$ for each value of $x$, weighted according to the probability of getting that particular $x$. That is

$$
H_{x}(y)=-\sum_{i, j} p(i, j) \log p_{i}(j)
$$

This quantity measures how uncertain we are of $y$ on the average when we know $x$. Substituting the value of $p_{i}(j)$ we obtain

$$
\begin{aligned}
H_{x}(y) & =-\sum_{i, j} p(i, j) \log p(i, j)+\sum_{i, j} p(i, j) \log \sum_{j} p(i, j) \\
& =H(x, y)-H(x)
\end{aligned}
$$

or

$$
H(x, y)=H(x)+H_{x}(y)
$$

The uncertainty (or entropy) of the joint event $x, y$ is the uncertainty of $x$ plus the uncertainty of $y$ when $x$ is known.

6. From 3 and 5 we have

$$
H(x)+H(y) \geq H(x, y)=H(x)+H_{x}(y)
$$

Hence

$$
H(y) \geq H_{x}(y)
$$

The uncertainty of $y$ is never increased by the knowledge of $x$. It will be decreased unless $x$ and $y$ are independent events, in which case it is not changed.

Those properties, forming the base of information theory, will be important when studying generalized entropies and conditions to apply approximations on mutual information.

\subsubsection{Practical equations on Shannon Entropy}

In our modern computer society, the Shannon entropy is defined using $K=1$ and using the logarithm base 2 function, i.e., $\log _{2}(\cdot)$, providing the following base equations:

$$
\begin{aligned}
H(x) & \equiv-\sum p(x) \log p(x) \\
H(x, y) & \equiv-\sum p(x, y) \log p(x, y) \\
H(x \mid y) & \equiv-\sum p(x) \sum p(x \mid y) \log p(x \mid y)
\end{aligned}
$$

Where $H(x \mid y)$ and $p(x \mid y)$ are conditional entropies and probabilities, with a different notation from the one used by Shannon, where $H(x \mid y)=H_{y}(x)$ and $p(x \mid y)=p_{y}(x)$. 


\title{
1.2.3 Mutual Information
}

The original idea of MI is in Shannon studies of noisy channels, as he questions how to define the actual information being transmitted if we have errors due to noise:

\begin{abstract}
Suppose there are two possible symbols 0 and 1, and we are transmitting at a rate of 1000 symbols per second with probabilities $p_{o}=p_{1}=\frac{1}{2}$. Thus, our source is producing information at the rate of 1000 bits per second. During transmission the noise introduces errors so that, on the average, 1 in 100 is received incorrectly (a 0 as 1 , or 1 as 0 ). What is the rate of transmission of information? Certainly less than 1000 bits per second since about $1 \%$ of the received symbols are incorrect. Our first impulse might be to say the rate is 990 bits per second, merely subtracting the expected number of errors. This is not satisfactory since it fails to take into account the recipient's lack of knowledge of where the errors occur. We may carry it to an extreme case and suppose the noise so great that the received symbols are entirely independent of the transmitted symbols. The probability of receiving 1 is $\frac{1}{2}$ whatever was transmitted and similarly for 0 . Then about half of the received symbols are correct due to chance alone, and we would be giving the system credit for transmitting 500 bits per second while actually no information is being transmitted at all. Equally "good" transmission would be obtained by dispensing with the channel entirely and fipping a coin at the receiving point. (SHANNON; WEAVER, 1963, p. 20)
\end{abstract}

Finally, Shannon defines all the forms for the rate of transmission $R$ as:

$$
\begin{aligned}
R & =H(x)-H_{y}(x) \\
& =H(y)-H_{x}(y) \\
& =H(x)+H(y)-H(x, y)
\end{aligned}
$$

explaining:

\begin{abstract}
The first defining expression has already been interpreted as the amount of information sent less the uncertainty of what was sent. The second measures the amount received less the part of this which is due to noise. The third is the sum of the two amounts less the joint entropy and therefore in a sense is the number of bits per second common to the two. Thus, all three expressions have a certain intuitive significance.
\end{abstract}

As we call it today, this formulation and the concept of the common information between two sources sharing a channel evolved to the Mutual Information. To understand the relation, we can look at how (PAPOULIS, 1991) defines MI:

\footnotetext{
Mutual Information The function $I(\mathfrak{A}, \mathfrak{B})=H(\mathfrak{A})+H(\mathfrak{B})-H(\mathfrak{A} \cdot \mathfrak{B})$ $i$ called the mutual information of the partitions $\mathfrak{A}$ and $\mathfrak{B}$. From (15-49) it follows that $I(\mathfrak{A}, \mathfrak{B})=H(\mathfrak{A})-H(\mathfrak{A} \mid \mathfrak{B})=H(\mathfrak{B})-H(\mathfrak{B} \mid \mathfrak{A})$ Clearly $[$ see $(15-51)] I(\mathfrak{A}, \mathfrak{B}) \geq 0$ As we shall presently see, $I(\mathfrak{A}, \mathfrak{B})$ can be interpreted as the "information about $\mathfrak{A}$ contined in $\mathfrak{B}$ " and it equals the "information about $\mathfrak{B}$ contained in $\mathfrak{A} . "$

(PAPOULIS, 1991, p. 552)
} 
In the present work, we will use this concept from Papoulis, defining $I(\cdot)$, similar to $R(\cdot)$, as:

$$
I(x, y)=H(x)+H(y)-H(x, y)
$$

Also, we urge the reader to keep in mind this notion where " $I(x, y)$ can be interpreted as the information about $x$ contained in $y$, and it equals the information about $y$ contained in $x$ " (PAPOULIS, 1991). Hence, MI is the information shared mutually between two sources. It implies that having the information from a single source, $x$ implies having a part of the $y$ information, contained in $x$, which can be measured by $I(x, y)$. 
Figure 9 - MRI registration example
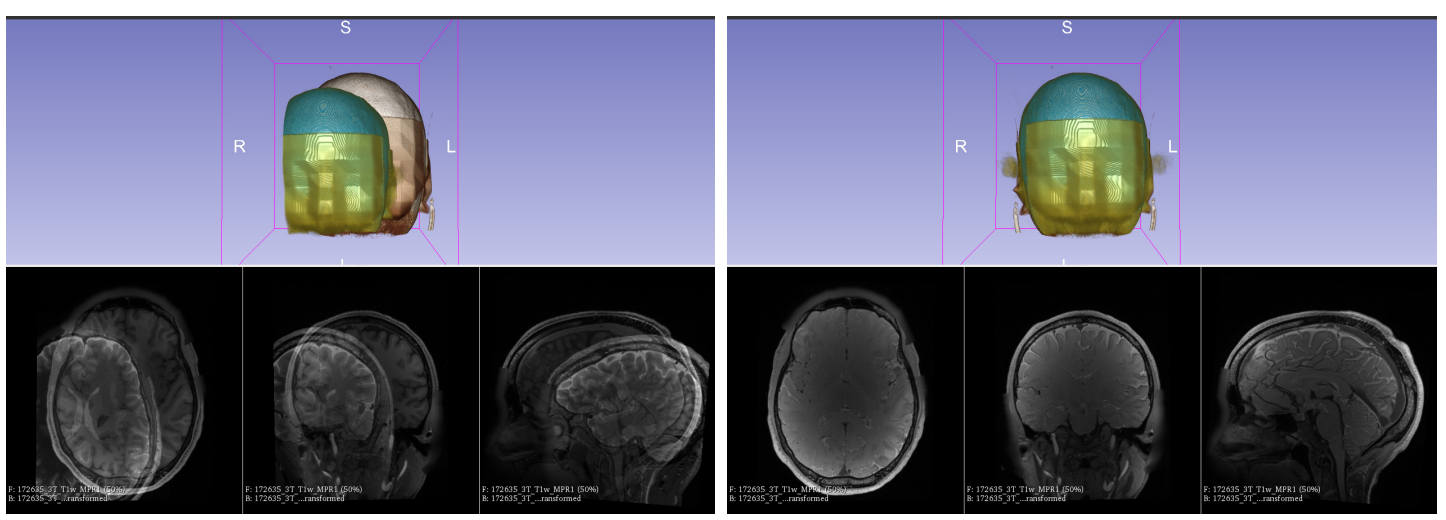

MRI registration using a single image and simulating another image by translating the first one. Source: author.

\title{
1.3 Image Registration
}

Image registration is the process of aligning images in the physical space, e.g., to combine multiple satellite images and provide a wider image, or, in our case, to align two medical images in order to enrich the information in a single image with the information from the other image.

From the Wikipedia entry:

\begin{abstract}
Image registration is the process of transforming different sets of data into one coordinate system. Data may be multiple photographs, data from different sensors, times, depths, or viewpoints. It is used in computer vision, medical imaging, military automatic target recognition, and compiling and analyzing images and data from satellites. Registration is necessary in order to be able to compare or integrate the data obtained from these different measurements.

(IMAGE..., 2021)
\end{abstract}

A single image is a set of information, as seen in sec. 1.1.2, providing a digital representation of a subset of the physical space. Registering two images combines the information provided by both images generating a new information source, e.g., a new image. The combination of information depends on what the registration aims to achieve and what information each image provides.

Registration must be done on the physical space, as the transformation parameters are related to physical operations. There is a relation to pixel, or voxel, coordinates and physical points in the space, given by the image spacing, center, and direction vector. Interpolation is then used to transport information from the digital space to the physical space, using multiple pixels to return an approximation of the image's value in a given physical coordinate.

Fig. 9 shows an example of medical image registration. The sources are two MRIs where the second is just translated to offset a few millimeters. When the software registers 
Figure 10 - Two examples of registration from the INPE website
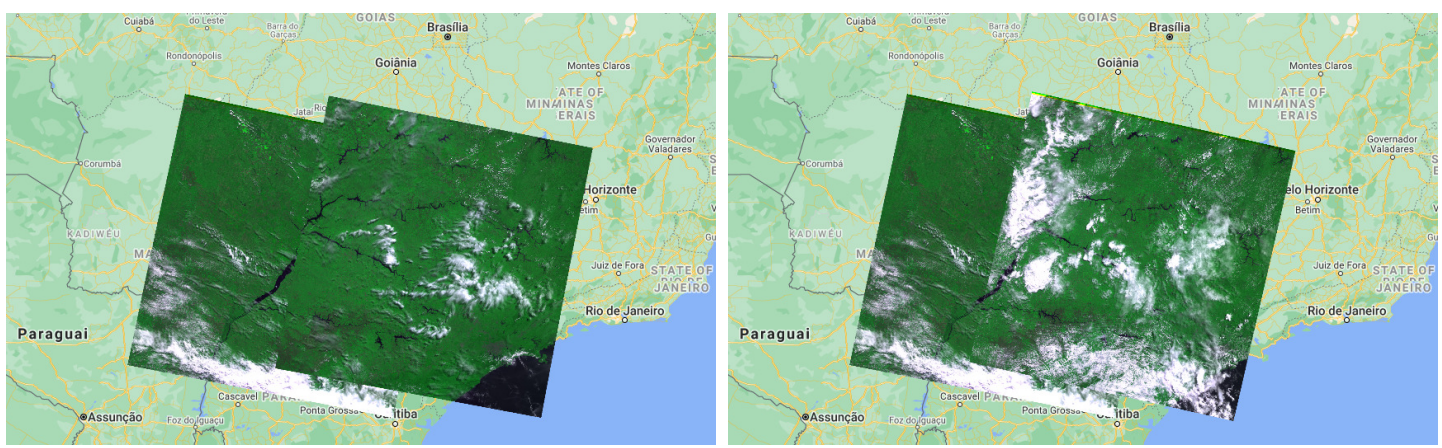

Two examples of registration from the INPE website, with the left being a bad one and the right a good one, the wrong registration can be seen on the river path breaking on the left one. Source: (C) 2019 - Catálogo do INPE < http://www2.dgi.inpe.br/catalogo/explore>

the images, it will align this offset, with both images perfectly overlapping and looking like a single image.

Fig. 10 shows a different situation, very common for image registration, where the images overlap is small (in Fig. 9 we have a complete overlap), extracted from INPE catalog website $^{1}$. In this situation, the overlap region is on the borders only. The registration generates a wider image than the originals, combining information from both sources to create a "virtual" image. In the left image, the registration was poor, and the river is broken where the images join. On the right, we can see a good registration where the river is continuous, but some clouds' artifacts are present only on one image, as they are from different moments in time.

\subsubsection{Registration Algorithm}

The registration process can be viewed as a combination of the four components, as defined by (BROWN, 1992):

Feature Space extracts information from both images.

Search Space defines the space that contains the solution for our registration.

Search Strategy defines how we will find the solution.

Similarity Metric provides a signal of how good our proposed solution is.

We register two images simultaneously, with one of them being called the fixed image. It is our reference or target image. The other image is called the moving image. It is the image we will align to the fixed one. First, the registration algorithm generates a 
Figure 11 - Image registration block diagram

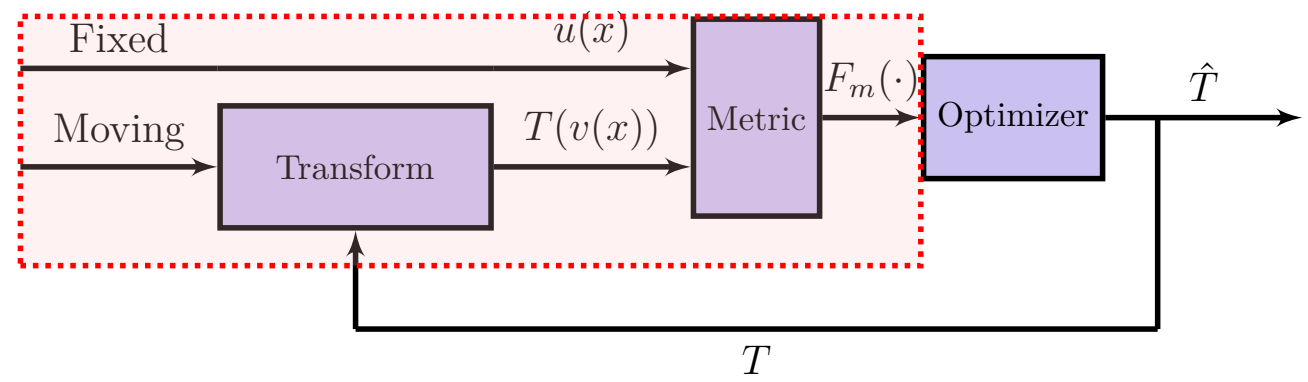

Source: author.

search space with all values of geometric transformations it will test. Then, using a search strategy, it sweeps this search space, checking at each step the similarity metric. When the similarity metric signals the alignment of fixed and moving images, the geometric transformation set is returned as the solution to register the images.

Mathematically, we try to solve the following equation, from (VIOLA; WELLS, 1997), to register images:

$$
\hat{T}=\underset{T}{\arg \max } F_{m}(u(x), v(T(x))
$$

Where $x$ is the registration points coordinates, $u(\cdot)$ and $v(\cdot)$ are the fixed and moving image functions, $T(\cdot)$ is the geometric transformation used to align the images, $F_{m}(\cdot)$ is the similarity metric used, also called cost function, $\underset{T}{\arg \max }$ is the argument that maximizes the metric, and $\hat{T}$ is the solution.

The process can also be viewed as a block diagram, shown in fig. 11, where we have both fixed and moving images entering the block, with the moving image passing a Transform block, generating a transformed image called $T(v(x))$. Both signals, $u(x)$ and $T(v(x))$, enter the Metric block, which analyzes those images for the similarity between them and returns a value, $F_{m}(\cdot)$. The Optimizer analyzes the $F_{m}(\cdot)$ signal and, using a search strategy, produces a new set of transformation parameters $T$. This new set $T$ will change the Transform output, $T(v(x))$, changing the Metric signal, $F_{m}(\cdot)$, creating a whole new iteration step. The process continues until the Optimizer decides the similarity signal is good enough and finishes providing a final set of transformation parameters, called $\hat{T}$, equals the last $T$ set generated.

\subsubsection{History of Medical Image Registration}

With the introduction of positron emission tomography (PET), new opportunities for medical diagnostic arise. By merging information from PET and CT, a better 
Figure 12 - Head fixation device
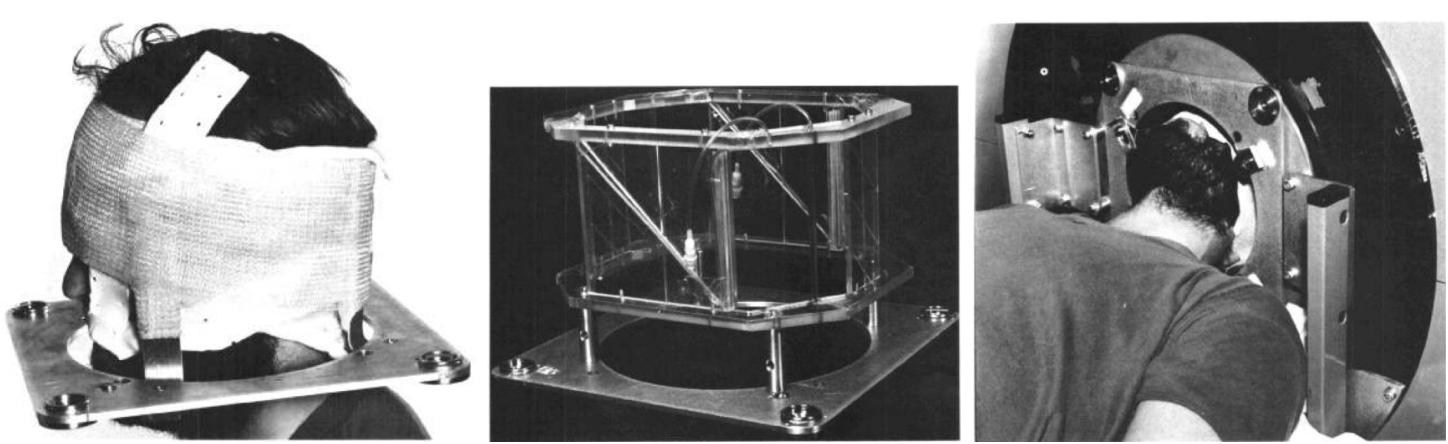

Source: (BERGSTRöM et al., 1981).

understanding of medical situations was possible:

With the introduction of positron emission tomography (PET), the potential of mapping functional changes in the brain was created. Because of the new type of information obtainable with PET, methods have been designed for stereotactic PET investigations and for the transfer of spatial information between $C T$ and PET.

(BERGSTRöM et al., 1981)

The approach proposed by (BERGSTRöM et al., 1981) was a head fixation device, shown in fig. 12, where the subject head was fixated, either by a glass fiber reusable mold or by aluminum bars, a procedure that takes about $30 \mathrm{~min}$. This head fixation apparatus was then mechanically attached to the medical scanners at the time, one for CT and the other for PET, providing a reference measurement between the two images and giving the parameters for the geometric transformation between the two images.

(MAGUIRE et al., 1986) proposed a correlation method for CT-PET registration, using landmarks manually marked on 2D slices of both images, they calculate a crosscorrelation coefficient used as a similarity metric. The work also remarks about the utilization of newly developed three-dimensional viewers to mark the landmarks in the 3D space. As noted by the authors, "Ideally, the choosing of the landmarks should be automated, but this is somewhat in the future for radionuclide type images" (MAGUIRE et al., 1986).

(PELIZZARI et al., 1989) proposed a more automated similarity metric by using computer matching surfaces:

This report describes a method of image correlation in three dimensions based on computer matching of surfaces but which does not suffer from the limitations mentioned above. Essentially, the surface of an anatomical structure visible in all scans of interest (most commonly the external surface of the head, including tissue) is used to define a patient-specific coordinate reference.

(PELIZZARI et al., 1989) 
The limitations cited by (PELIZZARI et al., 1989) were external markers, structures physically attached to the patient head that could be viewed on the images and acted as support elements for manual or automatic registration, and frames, as proposed by (BERGSTRöM et al., 1981).

(WOODS; CHERRY; MAZZIOTTA, 1992) proposed a method using statistical properties of images as a similarity metric:

The alignment techniques is based on the idealized assumption that if two
image sets are accurately aligned, then the value of any voxel in one image
set is related to the value of the corresponding voxel in the other image set
by a single multiplicative factor, R. If the image sets are misaligned, this
multiplicative factor is no longer constant but varies from voxel to voxel
throughout the image. The alignment algorithm systematically moves the
two image sets relative to one another until this voxel-to-voxel variation
is minimized.
(WOODS; CHERRY; MAZZIOTTA, 1992, 621)

(WOODS; CHERRY; MAZZIOTTA, 1992) algorithm is very interesting as it samples the voxels to calculate the statistical properties, starting sampling at an interval of 81 voxels, but as the registration progress the interval changes to $27,9,3$, and finally it calculates all voxels, succeeding to register rotations up to $40^{\circ}$ and translations up to 10 $\mathrm{mm}$. 


\title{
1.4 Geometric Transformation
}

Geometric Transformation is a function that maps some geometric structure into another structure. A good explanation is provided in the Wikipedia article about it:

\begin{abstract}
"A geometric transformation is a function whose domain and range are sets of points. Most often, the domain and range of a geometric transformation are both $\mathbb{R}^{2}$ or both $\mathbb{R}^{3}$. Often geometric transformations are required to be $1-1$ functions so that they have inverses." (GEOMETRIC..., 2021)
\end{abstract}

Generally, in image processing, geometric transformations are used to transform coordinates between two spaces, in our case, between the fixed and moving image spaces.

As seen on sec. 1.1.2, an image consists of a set of pixels, or voxels, arranged in a rectangular grid. In this way, each pixel or voxel has its position in space. When a figure is geometrically transformed, all pixels' positions are swept and filled with the value on the transformed position.

Let say we want to use a geometric transform $T(\cdot)$ to transform a $2 \mathrm{D}$ image, we have:

$$
\forall i, j: y(i, j)=x(T(i, j))
$$

where $x(\cdot)$ is the original image; $i$ and $j$ are coordinates on the 2D space; $x(i, j)$ and $y(i, j)$ represent the pixel of the image at coordinates $i, j ; T(\cdot)$ is a geometric transformation; and $y(\cdot)$ is the transformed image. This equation means we sweep all pixels available in the image $y$, and fill them with values from the image $x$ but with the position transformed.

\subsubsection{Types of transformation}

Geometric transformations can be divided into three groups:

Rigid does not change the object shape. Instead, it moves the object in the space performing translations and rotations along the axes. Formally it can be defined as a transformation that will not change the distance between any two points in the object, so its rigid name.

Affine changes the object's shape but preserves points, straight lines, and planes. It extends the former transformation by providing skewness and scaling transformations in the object.

Elastic transformations deform the object shape, not preserving geometric relations like points or straight lines. It is usually performed using $b$-splines grids that vary in number and complexity. 


\subsubsection{Translation}

To apply a geometric translation transformation, we add the translation offset vector to our point coordinates vector:

$$
\left[\begin{array}{l}
x_{1} \\
y_{1} \\
z_{1}
\end{array}\right]=\underbrace{\left[\begin{array}{l}
x_{0} \\
y_{0} \\
z_{0}
\end{array}\right]}_{P_{\text {point }}}+\underbrace{\left[\begin{array}{c}
t_{x} \\
t_{y} \\
t_{z}
\end{array}\right]}_{O_{\text {offset }}}
$$

\subsubsection{Matrix Transformation}

The transformations cited in sec. 1.4, except for the Elastic group, can all be made using a simple Transformation Matrix and an Offset Vector ${ }^{2}$. The identity transform can be defined as:

$$
\left[\begin{array}{l}
x_{1} \\
y_{1} \\
z_{1}
\end{array}\right]=\underbrace{\left[\begin{array}{lll}
1 & 0 & 0 \\
0 & 1 & 0 \\
0 & 0 & 1
\end{array}\right]}_{M_{\text {identity }}} \times \underbrace{\left[\begin{array}{l}
x_{0} \\
y_{0} \\
z_{0}
\end{array}\right]}_{P_{\text {point }}}+\underbrace{\left[\begin{array}{l}
0 \\
0 \\
0
\end{array}\right]}_{O_{\text {offset }}}
$$

\subsubsection{Rotation Matrices}

It's common to use Rotation Matrices to rotate an image within a Euclidean Space, this is the method used in the ITK library in the Matrix transformation ${ }^{3}$.

The most basic transformation is a simple rotation of an angle $\theta$ about one of the axes of the coordinate system ( $x$ axis in the example):

$$
R_{x}(\theta)=\left[\begin{array}{ccc}
1 & 0 & 0 \\
0 & \cos \theta & -\sin \theta \\
0 & \sin \theta & \cos \theta
\end{array}\right]
$$

So in this situation, if we want to map a point $P$ of our source image, represented as a vector, into a new point of the destination image, we perform the multiplication of

$\overline{2}$ There is an option to use a single matrix with an extra dimension to accommodate the offset vector on it, the usage depends on the efficiency of the system used.

3 All these functions are listed in the inheritance diagrams of the itk: : MatrixOffsetTransformBase class at < https://itk.org/Doxygen/html/classitk_1_1MatrixOffsetTransformBase.html> 
the matrix defined at eq. 1.7 and the point:

$$
\begin{aligned}
R_{x}(\theta) \times P & =\left[\begin{array}{ccc}
1 & 0 & 0 \\
0 & \cos \theta & -\sin \theta \\
0 & \sin \theta & \cos \theta
\end{array}\right] \times\left[\begin{array}{l}
x \\
y \\
z
\end{array}\right] \\
& =\left[\begin{array}{c}
x \\
y \cos \theta-z \sin \theta \\
y \sin \theta+z \cos \theta
\end{array}\right]=\left[\begin{array}{l}
x^{\prime} \\
y^{\prime} \\
z^{\prime}
\end{array}\right]
\end{aligned}
$$

Multiple rotations can be made using associative law. For example, if we like to rotate a point about the axis $x$ and later the axis $y$ we can use the $R_{y}(\phi)$ matrix and associate the operations generating the new $P^{\prime}$ point:

$$
\begin{gathered}
R_{y}(\phi)=\left[\begin{array}{ccc}
\cos \phi & 0 & \sin \phi \\
0 & 1 & 0 \\
-\sin \phi & 0 & \cos \phi
\end{array}\right] \\
P^{\prime}=R_{x}(\theta) \times R_{y}(\phi) \times P
\end{gathered}
$$

Using the associative property of matrix multiplication, you can compute the eq. 1.9 in two ways:

$$
\begin{aligned}
P^{\prime} & =R_{x}(\theta) \times\left[R_{y}(\phi) \times P\right] \\
P^{\prime} & =\left[R_{x}(\theta) \times R_{y}(\phi)\right] \times P
\end{aligned}
$$

The first form in eq. 1.10 will rotate the point $P$ about the $Y$ axis first and then about the $X$ axis later. The more interesting second form will produce another rotation matrix, combining the $R_{x}$ and $R_{y}$ matrices, and then rotate this point $P$ about this new combined axis.

This second form is a consequence of the Euler's Rotation Theorem: "In whatever way a sphere is turned about its center, it is always possible to assign a diameter, whose direction in the translated state agrees with that of the initial state." (EULER, 1776), more information can also be found at the Wikipedia page on this topic (EULER, ).

Since we need to transform multiple points or all points in the image when dealing with images, it makes sense to combine all transformations in a unique matrix and then apply this matrix to all points, saving a lot of unnecessary computation steps. 
In the case of the Eq. 1.9 we have:

$$
\begin{aligned}
R_{\text {sum }}(\theta, \phi)=R_{x}(\theta) \times R_{y}(\phi) & =\left[\begin{array}{ccc}
1 & 0 & 0 \\
0 & \cos \theta & -\sin \theta \\
0 & \sin \theta & \cos \theta
\end{array}\right] \times\left[\begin{array}{ccc}
\cos \phi & 0 & \sin \phi \\
0 & 1 & 0 \\
-\sin \phi & 0 & \cos \phi
\end{array}\right] \\
R_{\text {sum }}(\theta, \phi) & =\left[\begin{array}{ccc}
\cos \phi & 0 & \sin \phi \\
\sin \theta \sin \phi & \cos \theta & -\sin \theta \cos \phi \\
-\cos \theta \sin \phi & \sin \theta & \cos \theta \cos \phi
\end{array}\right]
\end{aligned}
$$

The final rotation matrix is the rotation matrix $R_{z}(\psi)$ over the $z$ axis:

$$
R_{z}(\psi)=\left[\begin{array}{ccc}
\cos \psi & -\sin \psi & 0 \\
\sin \psi & \cos \psi & 0 \\
0 & 0 & 1
\end{array}\right]
$$

\subsubsection{Rotation using roll, yaw, and pitch}

To create a geometric transformation using the roll, yaw, and pitch coordinates, we use:

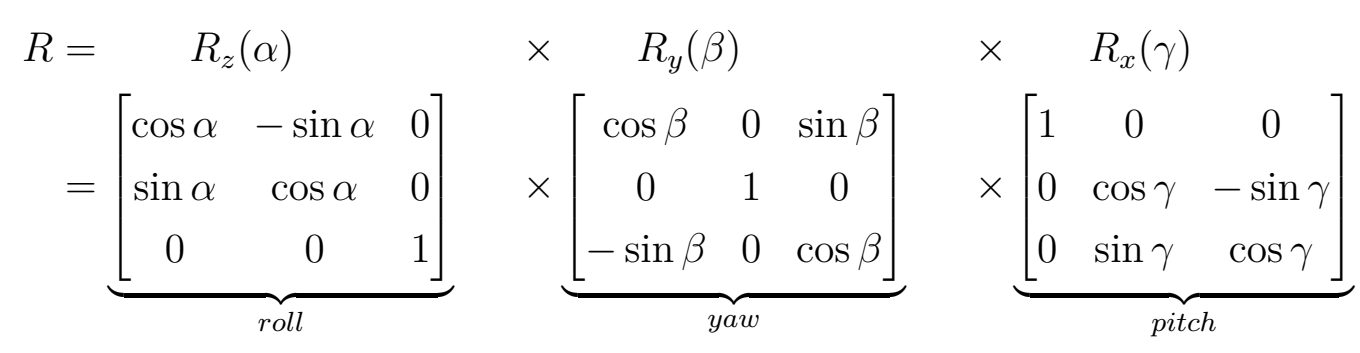

$$
R=\left[\begin{array}{ccc}
\cos \alpha \cos \beta & \cos \alpha \sin \beta \sin \gamma-\sin \alpha \cos \gamma & \cos \alpha \sin \beta \cos \gamma+\sin \alpha \sin \gamma \\
\sin \alpha \cos \beta & \sin \alpha \sin \beta \sin \gamma+\cos \alpha \cos \gamma & \sin \alpha \sin \beta \cos \gamma-\cos \alpha \sin \gamma \\
-\sin \beta & \cos \beta \sin \gamma & \cos \beta \cos \gamma
\end{array}\right]
$$

\subsubsection{Scaling Matrix}

To apply a geometric scaling transformation, we use the following matrix:

$$
\left[\begin{array}{l}
x_{1} \\
y_{1} \\
z_{1}
\end{array}\right]=\underbrace{\left[\begin{array}{ccc}
S_{x} & 0 & 0 \\
0 & S_{y} & 0 \\
0 & 0 & S_{z}
\end{array}\right]}_{M_{\text {scaling }}} \times \underbrace{\left[\begin{array}{c}
x_{0} \\
y_{0} \\
z_{0}
\end{array}\right]}_{P_{\text {point }}}
$$




\subsubsection{Skewness Matrix}

To apply a geometric skewness transformation, we use the following matrix:

$$
\left[\begin{array}{l}
x_{1} \\
y_{1} \\
z_{1}
\end{array}\right]=\underbrace{\left[\begin{array}{ccc}
1 & k_{y x} & k_{z z} \\
k_{x y} & 1 & k_{z y} \\
k_{x z} & k_{y z} & 1
\end{array}\right]}_{M_{\text {skewness }}} \times \underbrace{\left[\begin{array}{c}
x_{0} \\
y_{0} \\
z_{0}
\end{array}\right]}_{P_{\text {point }}}
$$

\subsubsection{Affine Matrix}

To fully develop an affine matrix for the geometric transformation, we can multiply the defined matrices and generate a single matrix.

The ITK library provides a transformation using versors, itk: :ScaleSkewVersor 3DTransform that seems to be more efficient. This algorithm also uses the concept of a quaternion (QUATERNIONS..., 2021).

With a set of parameters defined as:

$$
P(\underbrace{\theta, \phi, \psi}_{\text {rotation }}, \underbrace{t_{x}, t_{y}, t_{z}}_{\text {translation }}, \underbrace{s_{x}, s_{y}, s_{z}}_{\text {scaling }}, \underbrace{k_{y z}, k_{z z}, k_{x y}, k_{z y}, k_{x z}, k_{y z}}_{\text {skewness }})
$$

We need $\|(\theta, \phi, \psi)\| \leq 1$, if not we adjust with:

$$
\begin{aligned}
\epsilon & \leftarrow 1 \times 10^{-10} \\
n_{\text {factor }} & =\|(\theta, \phi, \psi)\| \times(1+\epsilon) \\
\theta & \leftarrow \theta / n_{\text {factor }} \\
\phi & \leftarrow \phi / n_{\text {factor }} \\
\psi & \leftarrow \psi / n_{\text {factor }} \\
\rho & \leftarrow \sqrt{\left(1-\|(\theta, \phi, \psi)\|^{2}\right)}
\end{aligned}
$$

With those auxiliary values set we can construct our affine matrix:

$$
M=\left[\begin{array}{ccc}
1-2\left(\phi^{2}+\psi^{2}\right)+\left(s_{x}-1\right) & 2(\theta \phi-\psi \rho)+k_{y z} & 2(\theta \psi+\phi \rho)+k_{z z} \\
2(\theta \phi+\psi \rho)+k_{x y} & 1-2\left(\theta^{2}+\psi^{2}\right)+\left(s_{y}-1\right) & 2(\phi \psi-\theta \rho)+k_{z y} \\
2(\theta \psi-\phi \rho)+k_{x z} & 2(\phi \rho+\theta \rho)+k_{y z} & 1-2\left(\theta^{2}+\phi^{2}\right)+\left(s_{z}-1\right)
\end{array}\right]
$$

Giving the final expression for calculating the full transformation:

$$
\underbrace{\left[\begin{array}{l}
x_{1} \\
y_{1} \\
z_{1}
\end{array}\right]}_{\text {transformed point }}=\underbrace{M}_{\text {affine matrix }} \times \underbrace{\left[\begin{array}{c}
x_{0} \\
y_{0} \\
z_{0}
\end{array}\right]}_{\text {original point }}+\underbrace{\left[\begin{array}{c}
t_{x} \\
t_{y} \\
t_{z}
\end{array}\right]}_{\text {translation }}
$$




\subsection{Interpolation}

When we use a geometric transformation, we start from a defined and existing point in the image, but the transformation does not always return an existing point. To better understand this, suppose a $2 \mathrm{D}$ image consisting of a square grid of 4 pixels, i.e., a dimension of $4 \times 4$ and isotropic images with a $1 \mathrm{~mm}$ spacing. Then we want a geometric translation of $0.3 \mathrm{~mm}$ on the $x$ axis. We will have the following:

$$
\underbrace{\left[\begin{array}{llll}
(0,0) & (0,1) & (0,2) & (0,3) \\
(1,0) & (1,1) & (1,2) & (1,3) \\
(2,0) & (2,1) & (2,2) & (2,3) \\
(3,0) & (3,1) & (3,2) & (3,3)
\end{array}\right]}_{\text {original coordinates }} \rightarrow \underbrace{\left[\begin{array}{lllll}
(0.3,0) & (0.3,1) & (0.3,2) & (0.3,3) \\
(1.3,0) & (1.3,1) & (1.3,2) & (1.3,3) \\
(2.3,0) & (2.3,1) & (2.3,2) & (2.3,3) \\
(3.3,0) & (3.3,1) & (3.3,2) & (3.3,3)
\end{array}\right]}_{\text {transformed coordinates }}
$$

The first pixel in the transformed image is filled with the original image value in position $(0.3,0)$, the second pixel with the position $(0.3,1)$, and so on. The problem with this situation is that we do not have a pixel at position $(0.3,0)$, so what value should be used to fill?

\subsubsection{Nearest Neighbor}

The simplest solution to the problem is the nearest neighbor interpolation. We use the closest pixel to the desired position or, as the name says, the nearest neighbor from the desired position.

In this example, using the position $(0.3,0)$, we have 4 possible neighbors: $(0,0)$, $(0,1),(1,0)$, and $(1,1)$. Calculating the distance between those 4 points, we finally have the nearest neighbor as $(0,0)$ and use the value at this position to fill.

The nearest neighbor is pretty simple to code and can be simplified to a simple rounding operation in some situations. Also, it has the advantage of not creating new values in the gray levels set, only copying values already on the image. However, the drawback of the algorithm is the low quality of the final image. This example shows that after calculating the nearest neighbor, our $0.3 \mathrm{~mm}$ translation did not affect the image. However, if we apply a transformation of $0.51 \mathrm{~mm}$, we would effectively translate $1 \mathrm{~mm}$.

\subsubsection{Linear interpolation}

A better algorithm and still very lightweight to code is linear interpolation, where we use the weighted average of the neighbors to calculate the fill value. The weight used in the average is the distance from the desired position to the neighbor. 
Figure 13 - Linear interpolation of a function

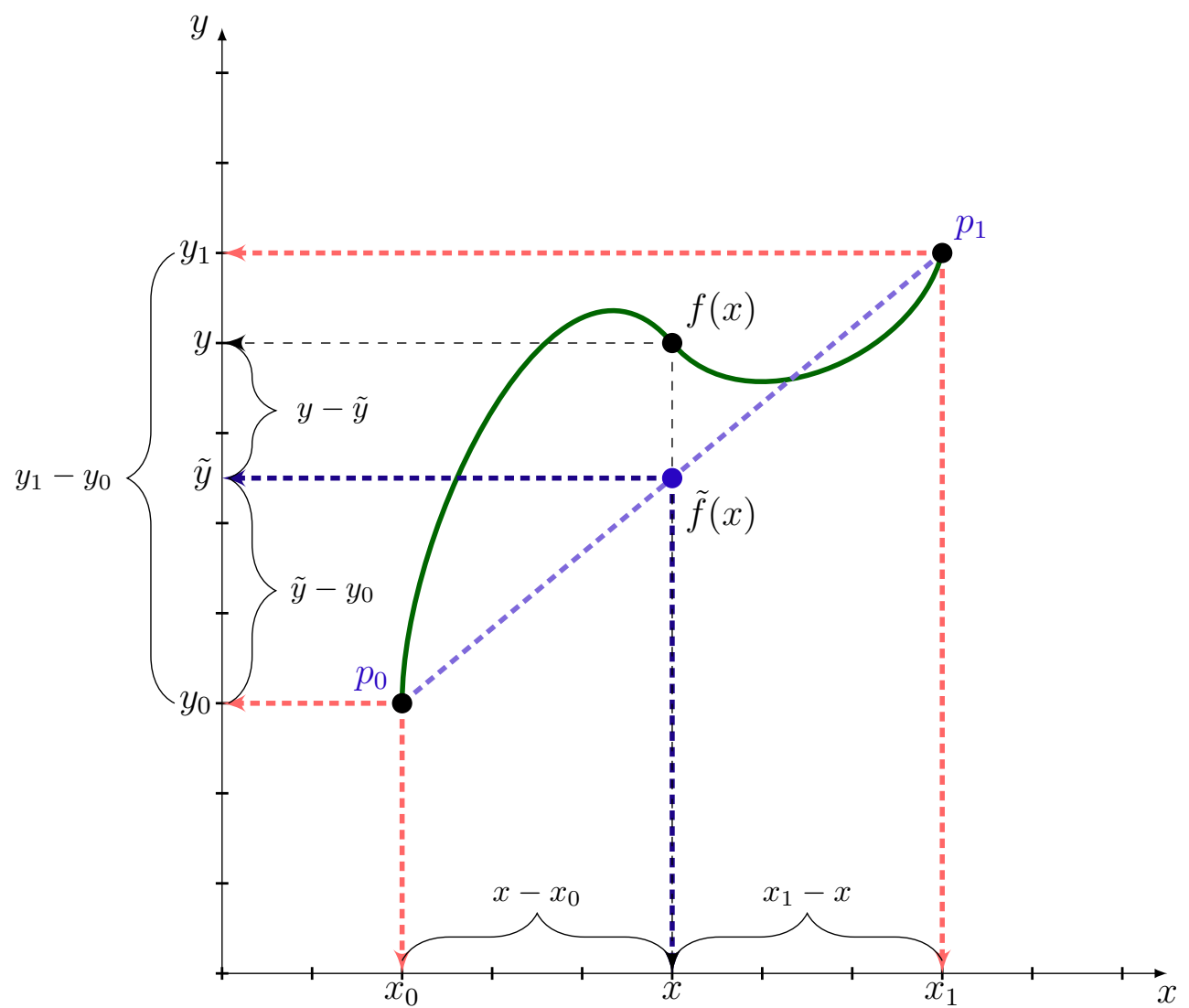

Linear interpolation of a function $f(x)$ (dark green) with a straight line $\tilde{f}$ (dashed blue) returning an approximation of $y$ called $\tilde{y}$. The error of the approximation is $y-\tilde{y}$. Source: author.

We start analyzing the problem of 1D linear interpolation, using fig. 13 we will call the $x$ axis the position of pixels, the $y$ axis as the pixel value, i.e., gray level, $(x, y)$ is our desired point with two neighbors, $p_{0}$ and $p_{1}$, so we have the following relation for the straight blue line:

$$
\frac{\tilde{y}-y_{0}}{x-x_{0}}=\frac{y_{1}-y_{0}}{x_{1}-x_{0}}
$$

Solving the equation for $\tilde{y}$ gives:

$$
\tilde{y}=y_{0}+\left(x-x_{0}\right) \frac{y_{1}-y_{0}}{x_{1}-x_{0}}=\frac{y_{0}\left(x_{1}-x\right)+y_{1}\left(x-x_{0}\right)}{x_{1}-x_{0}}
$$

We can also view the interpolation as the weighted average:

$$
\tilde{y}=y_{0}\left(1-\frac{x-x_{0}}{x_{1}-x_{0}}\right)+y_{1}\left(1-\frac{x_{1}-x}{x_{1}-x_{0}}\right)=y_{0}\left(\frac{x_{1}-x}{x_{1}-x_{0}}\right)+y_{1}\left(\frac{x-x_{0}}{x_{1}-x_{0}}\right)
$$


Figure 14 - Bilinear interpolation of a function.

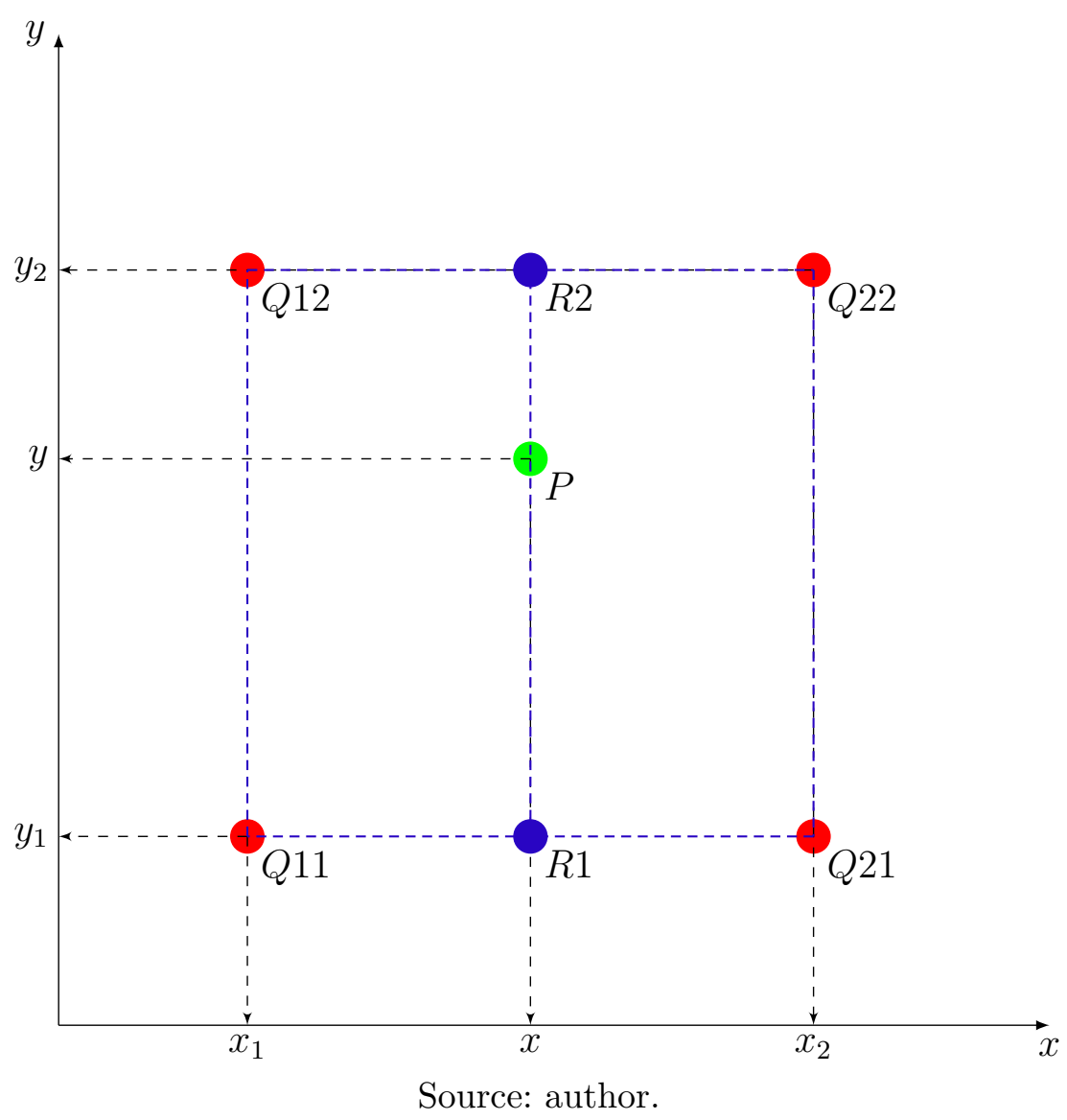

\subsubsection{Bilinear Interpolation}

The linear interpolation in a 2D space is called bilinear interpolation, and it can be viewed as a succession of two $1 \mathrm{D}$ interpolations stages. Using fig. 14, the first stage is to determine the two $1 \mathrm{D}$ interpolations solving points $R_{1}$ and $R_{2}$, and noting that now $y$ is an axis and the notation for the pixels value is $f(x, y)$ (BILINEAR. ., 2021):

$$
\begin{aligned}
& f\left(R_{1}\right)=f\left(x, y_{1}\right) \approx \frac{x_{2}-x}{x_{2}-x_{1}} f\left(Q_{11}\right)+\frac{x-x_{1}}{x_{2}-x_{1}} f\left(Q_{21}\right), \\
& f\left(R_{2}\right)=f\left(x, y_{2}\right) \approx \frac{x_{2}-x}{x_{2}-x_{1}} f\left(Q_{12}\right)+\frac{x-x_{1}}{x_{2}-x_{1}} f\left(Q_{22}\right) .
\end{aligned}
$$

Now, with those values for $R_{1}$ and $R_{2}$, we do another $1 \mathrm{D}$ interpolation to find $f(P)$ :

$$
f(x, y) \approx \frac{y_{2}-y}{y_{2}-y_{1}} f\left(R_{1}\right)+\frac{y-y_{1}}{y_{2}-y_{1}} f\left(R_{2}\right)
$$

Inserting the expressions for $f\left(x, y_{1}\right)$ and $f\left(x, y_{2}\right)$ into the above equation, we have a final formula for the 2D interpolation in a single step (BILINEAR..., 2021): 


$$
\begin{aligned}
f(x, y) & \approx \frac{y_{2}-y}{y_{2}-y_{1}} f\left(x, y_{1}\right)+\frac{y-y_{1}}{y_{2}-y_{1}} f\left(x, y_{2}\right) \\
& =\frac{y_{2}-y}{y_{2}-y_{1}}\left(\frac{x_{2}-x}{x_{2}-x_{1}} f\left(Q_{11}\right)+\frac{x-x_{1}}{x_{2}-x_{1}} f\left(Q_{21}\right)\right)+\frac{y-y_{1}}{y_{2}-y_{1}}\left(\frac{x_{2}-x}{x_{2}-x_{1}} f\left(Q_{12}\right)+\frac{x-x_{1}}{x_{2}-x_{1}} f\left(Q_{22}\right)\right) \\
& =\frac{\left(f\left(Q_{11}\right)\left(x_{2}-x\right)\left(y_{2}-y\right)+f\left(Q_{21}\right)\left(x-x_{1}\right)\left(y_{2}-y\right)+f\left(Q_{12}\right)\left(x_{2}-x\right)\left(y-y_{1}\right)+f\left(Q_{22}\right)\left(x-x_{1}\right)\left(y-y_{1}\right)\right)}{\left(x_{2}-x_{1}\right)\left(y_{2}-y_{1}\right)} \\
& =\frac{1}{\left(x_{2}-x_{1}\right)\left(y_{2}-y_{1}\right)}\left[\begin{array}{ll}
x_{2}-x & x-x_{1}
\end{array}\right]\left[\begin{array}{ll}
f\left(Q_{11}\right) & f\left(Q_{12}\right) \\
f\left(Q_{21}\right) & f\left(Q_{22}\right)
\end{array}\right]\left[\begin{array}{l}
y_{2}-y \\
y-y_{1}
\end{array}\right] .
\end{aligned}
$$




\subsection{Image Registration with Mutual Information}

Mutual Information, defined in sec. 1.2.3, can be used as a similarity metric in image registration.

Using the third form of eq. 1.3 with the notation $I(\cdot)$ for the MI, we have:

$$
I(x, y)=H(x)+H(y)-H(x, y)
$$

\subsubsection{Shannon Entropy Calculations for Images}

Using the images defined in sec. 1.1.2, we define the Shannon properties directly from the pixels or voxels:

$$
H(x)=\sum_{i=0}^{i_{\max }} p(i) \times \log p(i)
$$

Where $x$ is the information source related to the image, $i$ is the gray level intensity, ranging from 0 to $i_{\max }$, and $p(i)$ is the probability of the gray level $i$ in the image.

And the equation:

$$
H(x, y)=\sum_{i=0}^{i_{\max }} \sum_{j=0}^{j_{\max }} p(i, j) \times \log p(i, j)
$$

Where $x$ and $y$ are image information sources, $i$ is the gray level related to $x$, from 0 to $i_{\text {max }}, j$ is the gray level related to $y$, from 0 to $j_{\text {max }}, p(i, j)$ is the probability of a gray level $i$ on $x$ and $j$ on $y$ at the same position.

\subsubsection{Mutual Information for Registration}

Using the entropy functions defined in images properties, we have:

$$
I(x, y)=\underbrace{\sum_{i=0}^{i_{\max }} p(i) \times \log p(i)}_{H(x)}+\underbrace{\sum_{i=0}^{i_{\max }} p(i) \times \log p(i)}_{H(y)}-\underbrace{\sum_{i=0}^{i_{\max }} \sum_{j=0}^{j_{\max }} p(i, j) \times \log p(i, j)}_{H(x, y)}
$$

\subsubsection{Mathematical Analysis}

In theory, when we align an image, the information on any image used should not change. Thus, the fixed image remains the same, and the transformation used in the moving image is a reversible process, i.e., $v(x)=T^{-1}(T(v(x)))$, and do not change 
the image information. In this way, only the third part of eq. 1.19 should change, as the alignment change between $x$ and $y$ only changes $p(i, j)$ values.

We can analyze the partial derivative of the MI, $I(\cdot)$, concerning changes in the transformation parameters $T$, using the conditions that $p(i)$ and $p(j)$ do not change during the registration:

$$
\begin{aligned}
\frac{\partial I(x, y)}{\partial T} & =\frac{\partial H(x)}{\partial T}+\frac{\partial H(y)}{\partial T}-\frac{\partial H(x, y)}{\partial T} \\
& =\frac{\partial \sum_{i} p(i) \log p(i)}{\partial T}+\frac{\partial \sum_{j} p(j) \log p(j)}{\partial T}-\frac{\partial \sum_{i, j} p(i, j) \log p(i, j)}{\partial T} \\
& \approx \frac{\frac{\partial \sum_{i} p(i) \log p(i)}{\partial T}+0}{0}+\frac{\partial \sum_{j} p(j) \log p(j)}{\partial T}-\frac{\partial \sum_{i, j} p(i, j) \log p(i, j)}{\partial T}
\end{aligned}
$$

Giving an approximation for the Mutual Information derivative, that can be used under some conditions:

$$
\frac{\partial I(x, y)}{\partial T} \approx-\frac{\partial \sum_{i, j} p(i, j) \log p(i, j)}{\partial T}
$$

\subsubsection{Information loss during registration}

Eq. 1.24 is valid as long as $\frac{\partial H(x)}{\partial T}=0$ and $\frac{\partial H(y)}{\partial T}=0$. The first condition on $H(x)$ is pretty straight, as the fixed image does not change with $T$ 's changes, it's derivative should be zero.

The second condition, on $H(y)$, is more delicate. In theory, the geometric transformation is reversible. Making a reversible transformation over a source $y$ should not change the information quantity related to it. We are not creating or destroying information, just transforming, in a reversible way.

In practice, those geometric transformations occur on the digital domain, where we have limitations to possible voxel values, i.e., gray level values. The gray levels are quantized in a binary notation, either an integer or floating-point format, that cannot represent all values presented on the physical domain, a more analog one. Another limitation is that we have a finite set of voxels in an image, giving a finite resolution. The voxel grid distortion caused by this finite set on the transformed shape impacts the geometric transformation.

Fig. 15 help us understand the problem of digital domain, fig. 15a is a $50 \times 50$ pixel image, lets imagine it represents an area of $50 \times 50 \mathrm{~mm}^{2}$, where each pixel is a square of $1 \times 1 \mathrm{~mm}$. If we scale this image in a transformation $50 \times 50 \mathrm{~mm}^{2} \rightarrow 49 \times 49 \mathrm{~mm}^{2}$, it will be a reversible operation, and we can just transform back using a $49 \times 49 \mathrm{~mm}^{2} \rightarrow 50 \times 50 \mathrm{~mm}^{2}$ 
Figure 15 - Effects of geometric transformation and interpolation on an image
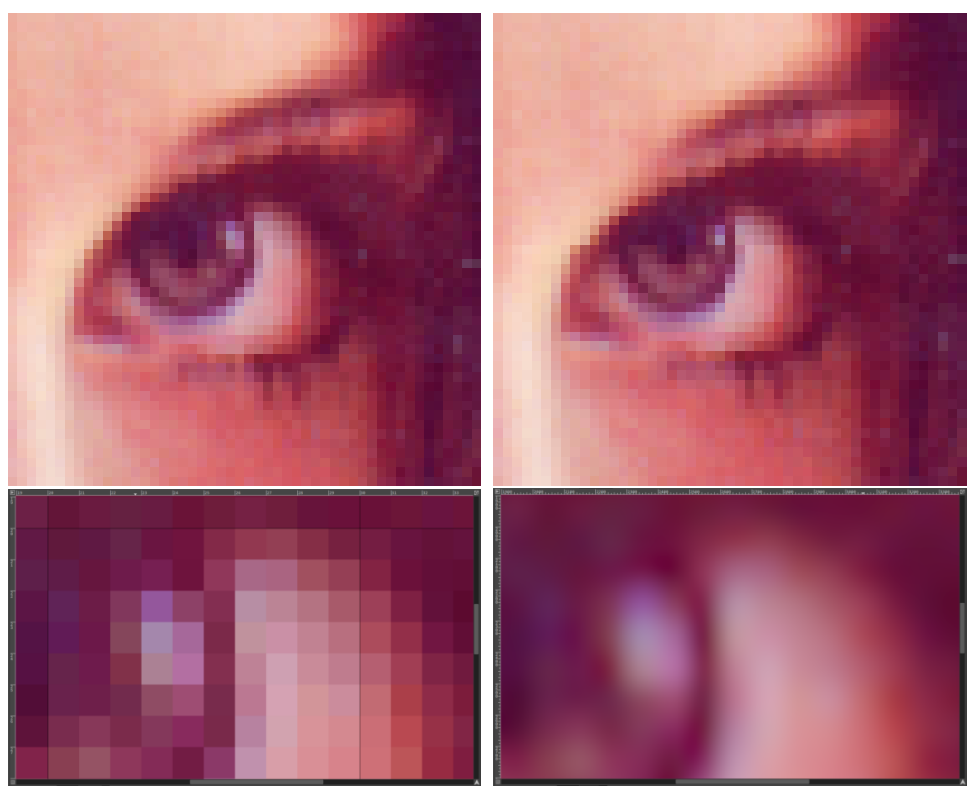

Effects of geometric transformation and interpolation on an image: (a) Lenna eye $(50 \times 50 \mathrm{px}$ detail of original image) (b) Lenna eye restored to 50px after reduction to 49px (c) detail of eye (d) after increase to $50000 \times 50000$ px with bicubic interpolation. Source: author.

transformation. But the grid used to represent our image is set as squares of $1 \times 1 \mathrm{~mm}^{2}$, saving this representation with $49 \times 49$ pixels, as shown in fig. $15 \mathrm{~b}$, lost information in a irreversible way, the information lost is obvious in the eye's iris.

Interpolation adds artifacts, i.e., artificial information not present in the original image. An example of the artifact is in fig. $15 \mathrm{c}-\mathrm{d}$, with (d) being an $\times 1000$ upscaled version of (c) using bicubic interpolation. This example may be seen good as a visualization helper. It smooths the pixel's values making a more believable reality to our brain. Also, it introduces many pixel values not present on the original image, changing the histogram function, from where the $p(\cdot)$ 's values come.

This means that, while in the mathematical domain we have $T(x) T^{-1}(x)=I(x)$, meaning that transforming and using the transform inverse right after it gives us an identity function, i.e., $I(x)$, in the digital domain this relation may not be true. The function $T(x)$ only maps points in the physical space, and to use this in images we need to map those points to the pixels or voxels in the image, a finite set.

\subsection{Classical $\mathrm{MI}$ registration papers}

Although not technically Mutual Information, (COLLIGNON et al., 1995b) was the first, as far as we know, to use the joint entropy as a similarity metric. As seen on eq. 1.19 and sec. 1.6.2 the significant contribution to changes in MI is the third term, $H(x, y)$, as this is the term that changes when we change the transformation parameters 
$T$.

A list of published works on MI follows, with a summary of the capture range results from each work:

(VIOLA; WELLS, 1997) proposed the first MI algorithm, named EMMA, evaluating it on 2D MRI slices. EMMA registration was successful in 50 randomly generated translations up to 32 pixels, rotations of $28^{\circ}$, and scaling up to $20 \%$.

(WELLS et al., 1996) obtained a success rate varying from $100 \%$ for $10 \mathrm{~mm}$ translations and $10^{\circ}$ rotations, dropping to $90 \%$ for $20 \mathrm{~mm}$ and $20^{\circ}, 68 \%$ for $20 \mathrm{~mm}$ and $68^{\circ}$ and finally $41 \%$ for $100 \mathrm{~mm}$ and $20^{\circ}$, concerning MR-CT registration.

(COLLIGNON et al., 1995a) showed similar results, i.e., up to $25 \mathrm{~mm}$ translations, and $11^{\circ}$ rotations, recovered fully automatically.

(MAES et al., 1997) reported registrations for up to $10^{\circ}$ rotations, and $40 \mathrm{~mm}$ translations, by tuning the parameter optimization order.

\subsubsection{Ml derived works in literature}

(STUDHOLME; HILL; HAWKES, 1999) introduced the normalized MI (NMI) with success up to $30 \mathrm{~mm}$ and $30^{\circ}$ when using smaller fields of view.

(RUECKERT et al., 2000) proposed higher-order MI to use spatial information but without details of the capture range used in the experiments.

(PLUIM; MAINTZ; VIERGEVER, 2000) introduced combined MI and gradient information to add spatial information to MI, showing promising results for the $15 \mathrm{~mm}$ and $15^{\circ}$ transforms. Further improvements were achieved for MR-T1/PET registration, although those improvements did not reflect a median or maximum error in most applications.

(RUSSAKOFF et al., 2004) introduced the regional MI (RMI) in 2004 and compared results to (PLUIM; MAINTZ; VIERGEVER, 2000; RUECKERT et al., 2000), and classical MI. RMI showed to be more robust with a success rate above $85 \%$ in target registration errors (TRE) with transforms up to $15 \mathrm{~mm}$. The study approached RMI as a translation cost function, showing good results in a range up to $60 \mathrm{~mm}$, but with an abnormal asymmetrical shape.

(LOECKX et al., 2010) introduced conditional MI (cMI), which improves the MI results for nonrigid B-spline registration. However, the algorithm demands up to 37 times more computational time than conventional MI. 
(ZHUANG et al., 2011) introduced spatially encoded mutual information (SEMI) and tested it with free-form deformation improving the robustness in non-uniformity fields compared to NMI.

(CHEAH; SHANMUGAM; MANN, 2012) introduced a combination of intensity and gradient information (CMI) with excellent results for up to $60 \mathrm{~mm}$ and $60^{\circ}$.

(PRADHAN; PATRA, 2016) introduced enhanced mutual information (EMI) using ranges up to $10 \mathrm{~mm}$ and $20^{\circ}$ with better results than NMI and RMI. However, their measure as a cost function for translation shows a local maximum in the x-axis. 
Figure 16 - Fractal shape of the Lake Mead, USA

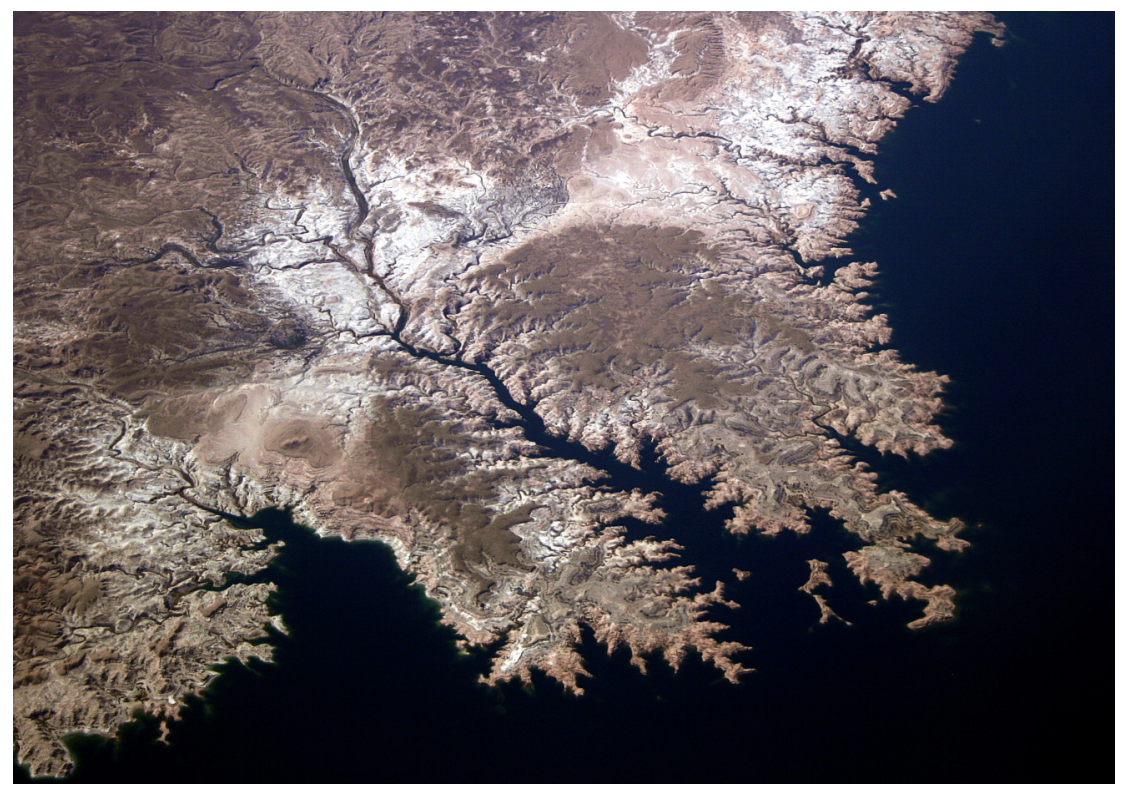

Source: @ Chris Moran (2010), available at <https://www.flickr.com/photos/buggs_moran/ $4516938146 />$.

\subsection{Fractal Dimensions}

Benoit Mandelbrot in 1967 wrote in his paper on fractal dimensions, "How Long Is the Coast of Britain? Statistical Self-Similarity and Fractional Dimension", the following abstract:

"Geographical curves are so involved in their detail that their lengths
are often infinite or, rather, undefinable. However, many are statistically
"self-similar", meaning that each portion can be considered a reduced-
scaling image of the whole. In that case, the degree of complication can
be described by a quantity D that has many properties of a "dimension",
though it is fractional; that is, it exceeds the value unity associated with
the ordinary, rectifiable, curves."
(MANDELBROT, 1967)

Fractal dimensions happen when there is a self-similarity in the object, where a part of the object is similar to the whole object in a statistical sense. An example of this is fig. 16, where the landmasses' divisions show a fractal property, with each division having its divisions.

The more detailed you try to measure a coastline as this, the more length you have. In the words of Hugo Steinhaus, cited in Mandelbrot's paper:

"The paradox of length. Length is a discontinuous functional. This means in plain words that we can trace in the vicinity of any rectifiable arc $A$ another arc $A^{\prime}$ whose length exceeds an arbitrary, previously prescribed limit, or even is infinite. This fact is something more than a mathematical curiosity: it has practical consequences. When measuring the left bank of the Vistula on a school map of Poland, we get a length which is appreciably smaller than that read on a map 1 : 200000 . (...) The same difficulty 
arises when measuring such objects as contours of leaves or perimeters of plane sections of trees: the result depends appreciably on the precision of the instruments employed. This paradox of length is not to be confused with the fact that every measurement of physical quantities, such as areas, volumes, masses or forces, is liable to errors (...) The left bank of the Vistula, when measured with increased precision would furnish lengths ten, hundred and even thousand times as great as the length read off the school map."

(STEINHAUS, 1954)

The self-similarity of a fractal geometry explains this situation, as fractal structures have self-similar structures as one goes deep into their details. Thus, the problem may look like simply a question of measurement precision. However, it is not how precise one can measure, other structures will emerge on the object that will demand a more precise measurement, and this process goes indefinitely, as can be seen in fig. 17 .

The name "fractal" is because the dimension $D$ of the object is not an integer number but a fractional number. The reasoning is as follows: imagine three simple euclidean geometry objects, a line, a square, and a cube. If we measure the line with a stick equal to the line, we need a single measurement. Changing the stick size to $\frac{1}{3}$ will force us to use 3 measurements to measure fully. In the case of a square, if we use smaller squares to measure it and change from a square with equal size to a square with $\frac{1}{3}$ of the size, we will need 9 squares to measure fully. For the cube, using a cube measurement with equal size and changing to another with $\frac{1}{3}$ will force us to make 27 measurements. Calling the number of measurements as $N$, the scaling factor as $\epsilon$ and the dimension as $D$ we have:

$$
N=\epsilon^{-D}
$$

from where we can derive a formulation for $D$ :

$$
D=-\frac{\log N}{\log \epsilon}
$$

In our example we have, for the line, square and cube:

$$
\begin{gathered}
D_{\text {line }}=-\frac{\log N}{\log \epsilon}=-\frac{\log 3}{\log \frac{1}{3}}=1 \\
D_{\text {square }}=-\frac{\log N}{\log \epsilon}=-\frac{\log 9}{\log \frac{1}{3}}=2 \\
D_{\text {cube }}=-\frac{\log N}{\log \epsilon}=-\frac{\log 27}{\log \frac{1}{3}}=3
\end{gathered}
$$

For Euclidean geometry, the dimensions are an integer, as expected. 
Figure 17 - Mandelbrot fractals

(a)

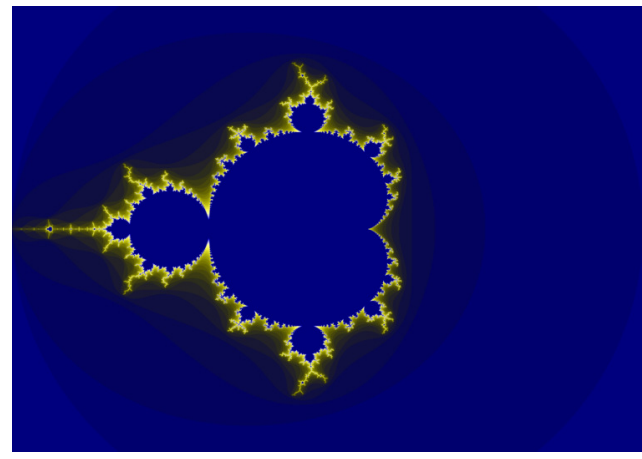

(c)

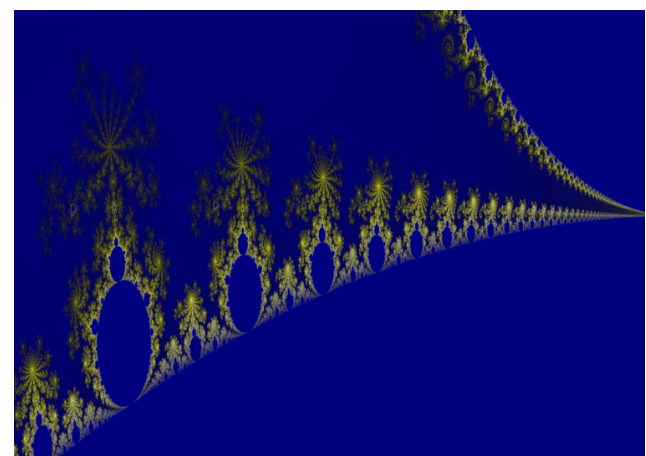

(e)

(b)

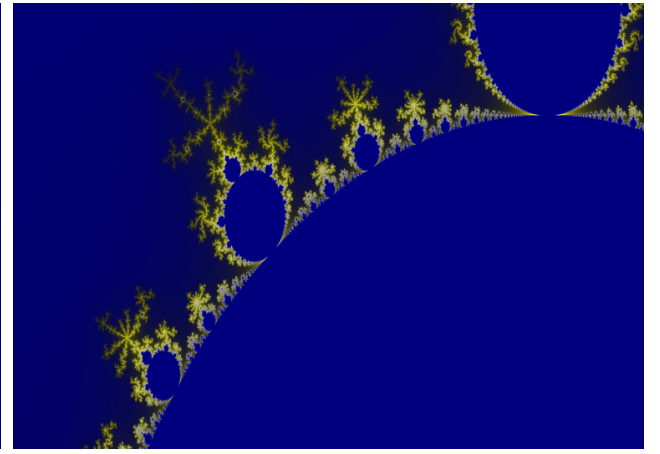

(d)

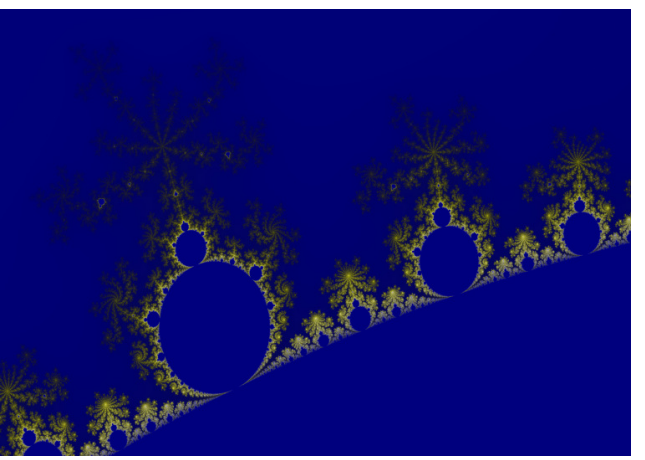

)

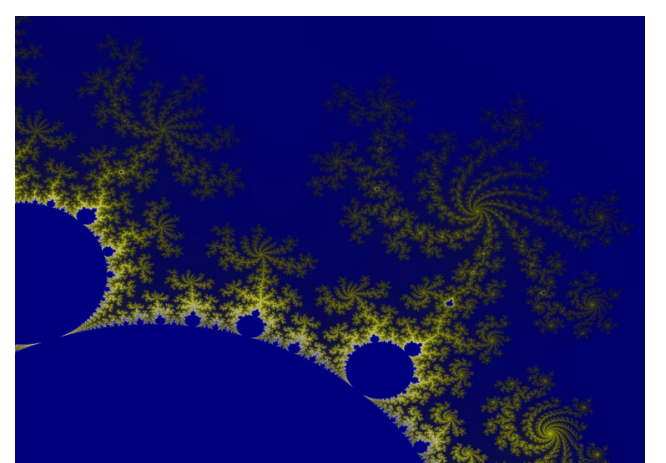

Mandelbrot fractals generated with the GIMP software, with various levels of zooming to show the fractal geometry property. Source: author. 
If we use a fractal structure, like the Koch curve, using the same method and reducing the measurement tool to $\frac{1}{3}$, we will have 4 measurements instead of the 3 in the euclidean case, giving now:

$$
D_{\text {Koch }}=-\frac{\log N}{\log \epsilon}=-\frac{\log 4}{\log \frac{1}{3}} \approx 1.2618595071429148 \ldots
$$

This means that the Koch curve has a fractal dimension, with $D \approx 1.2619$. 


\subsection{Generalized Entropies}

\subsubsection{Tsallis}

Constantino Tsallis defined his generalization of the Boltzmann-Gibbs (BG) entropy, or statistics, using concepts and structures from multi-fractals. Multi-fractals help understands some research areas where standard statistics are not well suited, e.g., nonlinear dynamical systems and long-range interaction systems. The Boltzmann-Gibbs (BG) entropy is the basis for thermal statistics and one of the pillars of modern science. However, BG statistics have some limitations and can be viewed as an approximation of nature under some conditions, in the words of Tsallis:

It seems that BG statistics satisfactorily describes nature if the effective microscopic interactions are short-ranges (i.e., close spatial connections) and the effective microscopic memory is short-ranges (i.e., close time connections) and the boundary conditions are non(multi)fractal. (TSALLIS, 1999)

To better suit those systems limited by BG statistics, Tsallis defined his statistics:

Multi fractal concepts and structures are quickly acquiring importance in many active areas of research (e.g., nonlinear dynamical systems, growth models, commensurate/incommensurate structures). This is due to their utility as well as to their elegance. Within this framework, the quantity that is normally scaling is $p_{i}^{q}$, where $p_{i}$ is the probability associated with an event and $q$ is any real number. I shall use this quantity to generalize the standard expression of the entropy $S$ in information theory, namely $S=-k \sum_{i=1}^{W} p_{i} \ln p_{i}$, where $W \in \mathbb{N}$ is the total number of possible (microscopic) configurations and $\left\{p_{i}\right\}$ are the associated probabilities. (TSALLIS, 1988)

Tsallis postulate the equation for his entropy:

$$
S_{q} \equiv k \frac{1-\sum_{i=1}^{W} p_{i}^{q}}{q-1}
$$

Where $q$ is the entropic index, $k$ is a conventional positive constant and $\sum_{i=1}^{W} p_{i}=1$. An alternative form of eq. 1.28, also defined in (TSALLIS, 1988) is:

$$
S_{q} \equiv \frac{k}{q-1} \sum_{i=1}^{W} p_{i}\left(1-p_{i}^{q-1}\right)
$$

Tsallis' entropy has a particular case where $q=1$, returning the BG statistics in this case: 


$$
\begin{aligned}
S_{1} \equiv \lim _{q \rightarrow 1} S_{q} & =\lim _{q \rightarrow 1} k \frac{1-\sum_{i=1}^{W} p_{i}^{q}}{q-1} \\
& =\lim _{q \rightarrow 1} \frac{k}{q-1} \sum_{i=1}^{W} p_{i}\left(1-p_{i}^{q-1}\right) \\
& =k \lim _{q \rightarrow 1} \frac{1-\sum_{i=1}^{W} p_{i} \exp \left[(q-1) \ln p_{i}\right]}{q-1} \\
& =-k \sum_{i=1}^{W} p_{i} \ln p_{i}
\end{aligned}
$$

The main difference between BG and Tsallis statistics lies in exponential functions in the core of Tsallis' eq. 1.28. Since we use normalized probabilities, $p_{i}$ will be in the range $0 \leq p_{i} \leq 1$ giving two possibilities, depending on $q$ (TSALLIS, 1999):

$$
\begin{array}{ll}
\text { for } q>1: & p_{i}^{q}<p_{i}, \\
\text { for } q<1: & p_{i}^{q}>p_{i},
\end{array}
$$

Where $q<1$ will privilege rare events, while $q>1$ privileges frequent events, allowing us to change the individual contribution of each event's possible value to the final entropy.

The $q$ parameters in the Tsallis' eq. 1.28, called the entropic index, also relate to the fractal dimension being analyzed. One of the first examples of this relation between $q$ and the fractal dimension can be found in (ALEMANY; ZANETTE, 1994), where studies on Lévy flights found a relation between the entropic index $q$ and the Lévy flight diffusion constant $\gamma$, where $q=\frac{3+\gamma}{1+\gamma}$ "fixes the generalized statistics compatible with the fractal dimension $\gamma$ " (ALEMANY; ZANETTE, 1994).

Tsallis, in his work on discrimination between two hypotheses, noted the need for a good entropic index choice: "It is expected that, for every specific use, better discrimination will be achieved with appropriate ranges of values of $q$ "(TSALLIS, 1998).

Tsallis defines the joint entropy for two independent systems, due to the pseudoadditivity, as: (TSALLIS, 1994a)

$$
H_{q}(x, y)=H_{q}(x)+H_{q}(y)+(1-q) H_{q}(x) H_{q}(y)
$$

\subsubsection{Tsallis q-logarithm and q-exponential}

(TSALLIS, 1994b) also defines two auxiliary functions to deal with his entropy: 


$$
\begin{aligned}
\ln _{q} x & \equiv \frac{x^{1-q}-1}{1-q} \\
e_{q}^{x} & \equiv[1+(1-q) x]_{+}^{\frac{1}{1-q}}
\end{aligned}
$$

where $[z]_{+}=\max \{z, 0\}$, and noting that:

$$
\begin{aligned}
\lim _{q \rightarrow 1} \ln _{q} x & =\ln x \\
\lim _{q \rightarrow 1} e_{q}^{x} & =e^{x} \\
\ln _{q} e_{q}^{x}=e_{q}^{\ln x}= & x
\end{aligned}
$$

Equations 1.33 and 1.34 can be used to write eq. 1.28 in an alternative form using the q-logarithm function:

$$
S_{q} \equiv-k \sum_{i} p_{i}^{q} \ln _{q} p(i)
$$

\subsubsection{Tsallis Mutual Information}

Tsallis MI is introdued in (BORLAND; PLASTINO; TSALLIS, 1998) and is typically used in the literature as:

$$
I_{q}(x, y)=H_{q}(x)+H_{q}(y)-H_{q}(x, y)+(1-q) H_{q}(x) H_{q}(y)
$$

First, as noted by (FURUICHI, 2006), one should not use the term MI with Tsallis entropy as "proper evidence of channel coding theorem for information has not ever been shown in the context of Tsallis statistics".

(YAMANO, 2001) develops the generalized mutual information (GMI) equation as:

$$
I_{q}(Y ; X)=\frac{H_{q}(X)+H_{q}(Y)-H_{q}(X, Y)+(q-1) H_{q}(X) H_{q}(Y)}{1+(q-1) H_{q}(X)}
$$

which have an extra component in the denominator regarding equation (1.36).

(SPARAVIGNA, 2015) shown that the Yamano's equation is not symmetrical, i.e., $I_{q}(Y ; X) \neq I_{q}(X ; Y)$, and proposed a new equation: ${ }^{4}$

$$
I_{q}(Y ; X)=\frac{H_{q}(X)+H_{q}(Y)-H_{q}(X, Y)+(1-q) H_{q}(X) H_{q}(Y)}{1+(1-q) \max \left\{H_{q}(X), H_{q}(Y)\right\}}
$$

using the maximum between the two entropies at denominator.

4 The factors $(1-q)$ and $(q-1)$ are used in Yamano and Sparavigna equations differently. They are reproduced here as found in the published papers. However, the Yamano equation may have misplace this factor. 


\subsubsection{Rényi}

Alfréd Rényi defined his generalization of the Shannon entropy, in his work (RéNYI, 1961), as:

$$
H_{\alpha}(X)=\frac{1}{1-\alpha} \log \left(\sum_{i=1}^{n} p_{i}^{\alpha}\right)
$$

noting that $\alpha>0$ and $\alpha \neq 1$ satisfies the properties mentioned in sec. 1.2.1.2.

Also, as with Tsallis's, Rényi's entropy has a special case where $\alpha=1$ return the Shannon's entropy:

$$
\lim _{\alpha \rightarrow 1} H_{\alpha}(X)=-\sum_{i=1}^{W} p(i) \log p(i)
$$

\subsection{Nonextensivity}

\subsubsection{Physical properties}

Physical materials and systems possess physical properties. Those properties can be divided into two categories:

Intensive are properties intrinsic to the material or system and do not depend on the quantity or size, e.g., density, color, magnetic permeability, etc.

Extensive are properties of materials or systems that depend on the size, or extent, e.g., mass, volume, entropy, etc.

So it follows that Extensive systems have properties, e.g., mass, volume, entropy, that extends, augments, increment, as the systems grow. In simple situations, this is a valid approximation of reality.

\subsubsection{Nonextensitivy}

Nonextensivity is inherent in systems where long-range interactions or spatio-temporal complexity are present.

(LYRA; TSALLIS, 1998)

Long-range interactions mean that we have forces acting between particles in the opposite regions of our system, so we cannot approximate a particle's state very well without knowledge of distant particles.

Spatio-temporal complexity means that the space and time in and around our system are complex. Furthermore, this relates to long-range, since to understand a part of our space and time, we need to consider information on a massive number of particles. 
When we have several particles acting, the conventional approach is to deduce a field. This deduction was made with the $E$ electrical field, $H$ for the magnetic field, $G$ for the gravitational field. They are models of space and time, in many cases considered even as constant to help solve a problem. This approximation works because, in most cases, the long-range interaction is weak and negligible, e.g., the gravitational field produced by a distant planet like Neptune on Earth may be negligible compared to Earth and Moon fields.

Physics and Engineering see the problem from the point of view of interactions. The question is if we need to consider the interaction to understand a problem or not, as many interactions come from forces with a power law, vanishing with distance:

$$
\begin{aligned}
F & =G \frac{m_{1} m_{2}}{r^{2}} & \text { Newton's Law } \\
F & =k_{e} \frac{\left|q_{1} q_{2}\right|}{r^{2}} & \text { Coulomb's Law } \\
B(r) & =\frac{\mu_{0}}{4 \pi} \int_{C} \frac{I d l \times \hat{r}^{\prime}}{\left|r^{\prime}\right|^{2}} & \text { Biot-Savart's Law }
\end{aligned}
$$

The statistics have the concept of independent events, where one event does not affect the probability of occurrence of other events. This is the main difference of Extensive and Non-extensive physics, or statistics. In Extensive we accept that one event will not affect others. They can superpose without affecting others so that we can study each event separately. Non-extensive means that all the events may interact or even all interact, forcing us to study all events and all possible interactions, to understand a system under Non-extensive physics.

\subsubsection{Non-Additivity}

This has a clear relation to additivity, as we can add the parts of an Extensive system to solve it, and several Extensive systems can be added and be studied as a single Extensive system. Tsallis explains it much better:

"The traditional functional $S_{B G}$ is said to be additive. Indeed, for a system composed of any two (probabilistically) independent subsystems, the entropy $S_{B G}$ of the sum coincides with the sum of the entropies. The entropy $S_{q}(q \neq 1)$ violates this property, and is therefore non-additive. As we see, entropic additivity depends, from its very definition, only on the functional form of the entropy in terms of probabilities. The situation is generically quite different for the thermodynamic concept of extensivity. An entropy of a system or of a subsystem is said extensive if, for a large number $N$ of its elements (probabilistically independent or not), the entropy is (asymptotically) proportional to $N$. Otherwise, it is nonextensive. 
This is to say, extensivity depends on both the mathematical form of the entropic functional and the correlations possibly existing within the elements of the system. Consequently, for a (sub)system whose elements are either independent or weakly correlated, the additive entropy $S_{B G}$ is extensive, whereas the non-additive entropy $S_{q}(q=1)$ is nonextensive. In contrast, however, for a (sub)system whose elements are generically strongly correlated, the additive entropy $S_{B G}$ can be nonextensive, whereas the non-additive entropy $S_{q}(q \neq 1)$ can be extensive for a special value of $q$. "

(TSALLIS, 2009, preface, ix)

Tsallis explains, beautifully, the power of his additive entropy, eq. 1.28. As $S_{B G}$ can only explain some systems as Non-extensive systems, i.e., the sum of the parts is not equal the total, applying $S_{q}$ with $q \neq 1$ may explain the system in an Extensive way, allowing us to explain the total system as a sum of it parts. Tsallis entropy can "view" a Non-Extensive system, in classical Boltzmann-Gibbs entropy, as an Extensive system.

This can be seen as Tsallis entropy can better model physics of some systems, those systems with long-range interaction, spatio-temporal complexity, and fractal dimensions. 


\title{
2 Proposal
}

\author{
"Bold ideas, unjustified \\ anticipations, and speculative \\ thought, are our only means for \\ interpreting nature: our only \\ organon, our only instrument, \\ for grasping her."
}

Karl Popper, The Logic of Scientific Discovery

\subsection{Hypothesis}

Our central hypothesis is defined as:

"The use of generalized entropies on mutual information metrics can improve affine registration of brain images."

The principle of falsifiability can be easily determined for this hypothesis as we can determine a set of experiments, e.g., registration experiments, to try to contradict the hypothesis statement.

The concept of verifiability is hard to comply with since we cannot experiment on all possible brain images. With that in mind, we will try to design a set of experiments to understand how and where generalized entropies improve the metric function in a registration process.

\subsection{Rationale}

Section 1.10.3 showed that Tsallis entropy could "view" some Non-Extensive systems as Extensive systems.

To answer if the system that consists of the registration process of a brain image is a Non-Extensive system, we need to analyze some properties of this system.

\subsubsection{Brain Fractal Geometry}

The brain evolved into a convoluted structure to overcome limitations in brain size. This convoluted structure has a fractal dimension. 
It was an early evolutionary advantage to have a bigger brain since the more brain mass, the more functions a species could perform. In this way, it could have better vision, smelling, or hearing, making the individual more apt to survive.

\footnotetext{
"principle of proper mass: The mass of neural tissue controlling a particular function is appropriate to the amount of information processing involved in performing the function."

(JERISON, 1973, c.1, pg.8)
}

(HOFMAN, 1991) provides a solid argument about the brain fractal geometry and explains a little about why it happens:

\begin{abstract}
"Among the most distinctive morphological features of mammalian brains is the evolutionary expansion of the cerebral cortex. A consequence of this evolutionary phenomenon is that the geometry of the brain, and especially its surface area, has changed notably since the late Cretaceous." "Allometric analyzes of the cortical surface area and brain volume reveal that among mammals two major groups can be distinguished: (1) species with smooth, non-convoluted brains where the cortical surface area increases as the two-thirds power of brain volume, and (2) species with convoluted brains, where the cortical morphology is described by more complex mathematical models. The size of cortex at which this transition from a lissencephalic to a gyrencephalic brain takes place, i.e., at which convolutions appear at the cortical surface, is at a surface area of ca. 10 $\mathrm{cm}^{2}$, corresponding to a brain volume of $3-4 \mathrm{~cm}^{3} . "$ (HOFMAN, 1991)
\end{abstract}

Large mammals, with brain volume above $4 \mathrm{~cm}^{3}$, have a convoluted brain, i.e., a gyrencephalic brain. The surface of those brains is not smooth but convoluted, folded in structures called gyros. The advantage of a larger surface area is that most cortex neurons reside in a thin layer near the brain's surface, in the tissue called gray matter, with only a few on the inner tissue called white matter, which consists mainly of axons that primarily transmit information. This convoluted cortex is shown in fig. 18, with a bisected view of the brain.

If we look at the brain as a computer, a very sophisticated and evolved one, but still a system that processes information, the central part of this processing is done in the neurons at gray matter, being transmitted to other neurons or parts of the body, by the white matter. So, it is logical to assume that the more gray matter it has, the more neurons, and the more raw processing capacity ${ }^{1}$ it may have.

There were several studies on brain fractal geometry, highlighting the following respective dimensions: (HOFMAN, 1991) found $D=2.70 \pm 0.07$, (MAJUMDAR; PRASAD, 1988 ) found $D=2.60 \pm 0.05$, (CHUANG; VALENTINO; HUANG, 1991) found $D=2.204$, (FREE et al., 1996) found $D=2.30 \pm 0.01$, (KISELEV; HAHN; AUER, 2003) found $D=2.80 \pm 0.05$.

1 Raw capacity don't necessarily means better, since how the neurons are organized play a role in this processing, and not only the number of neurons, so we will call it raw capacity. 
Figure 18 - Human brain bisected in the sagittal plane
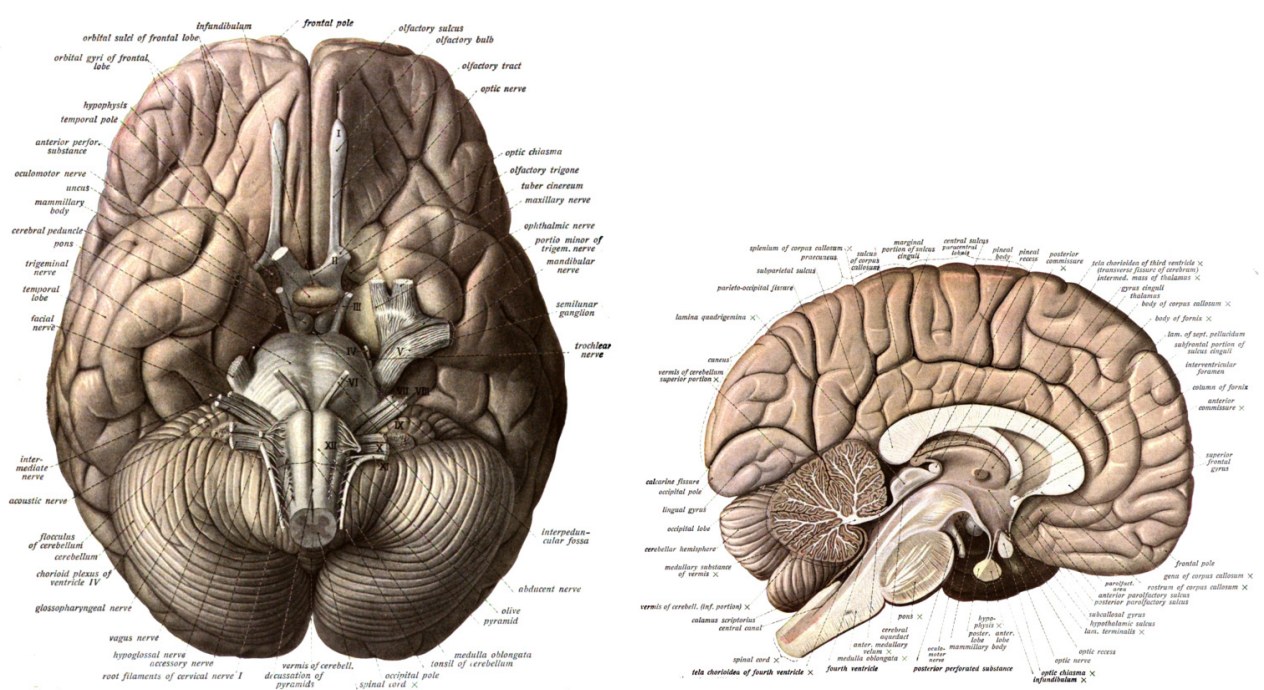

Source: Dr. Johannes Sobotta - Atlas and Text-book of Human Anatomy Volume III Vascular System, Lymphatic system, Nervous system and Sense Organs, Public Domain, <https:// commons.wikimedia.org/w/index.php?curid=29135452>.

Recently, (MARZI et al., 2020) published a study on brain fractal dimensions and how it changes with healthy aging (Fig. 19), where we can find some $D$ ranges, as $2.5<D<2.6$ for NKI2 dataset with ages from 6 to 18 years and $2.45<D<2.55$ for $20-90$ years.

The brain fractal dimension is well measured and defined in academic literature and can be viewed as an evolutionary step in creating more complex animals. 
Figure 19 - Brain Fractal Dimensions in relation to age

NKI2 dataset

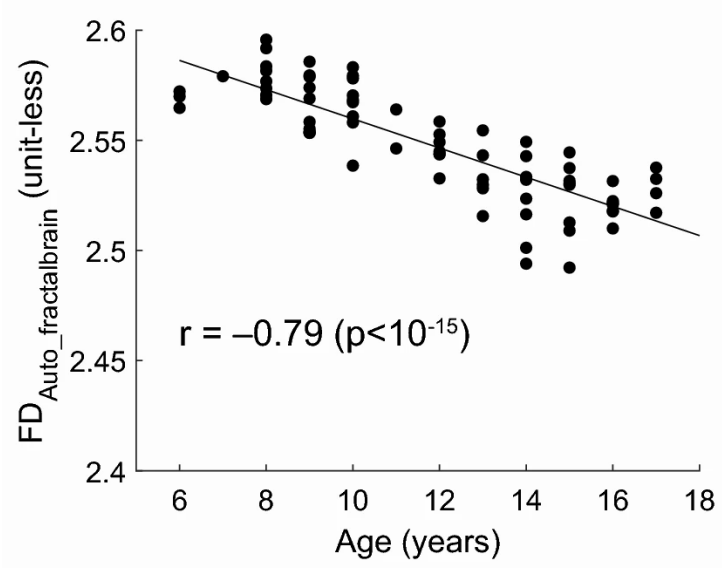

a

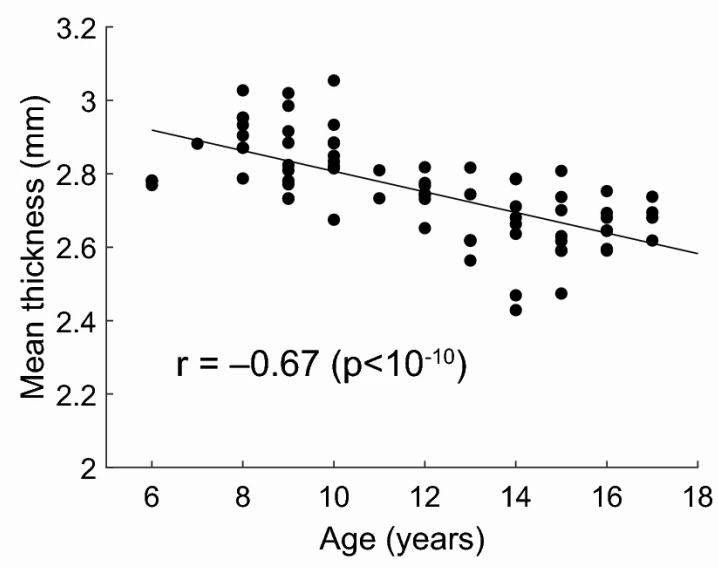

C

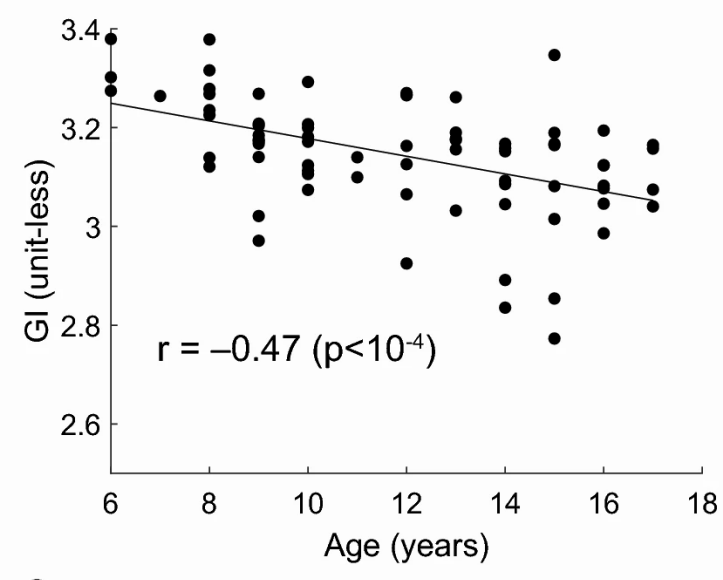

ICBM dataset

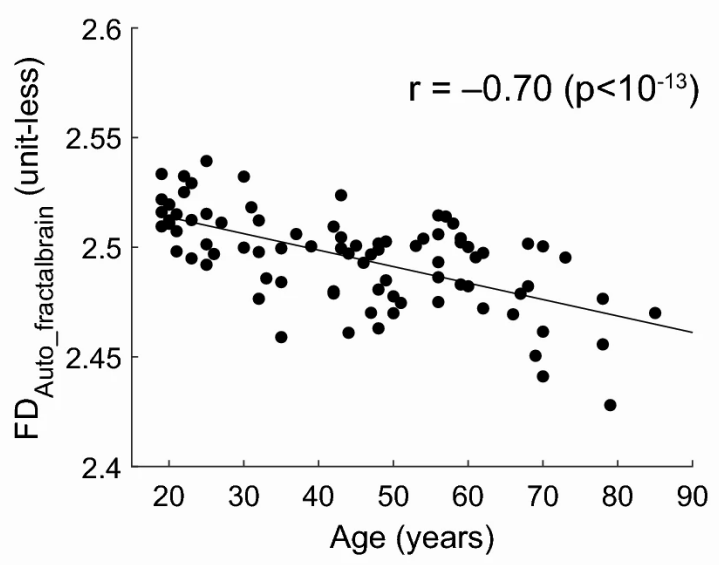

b

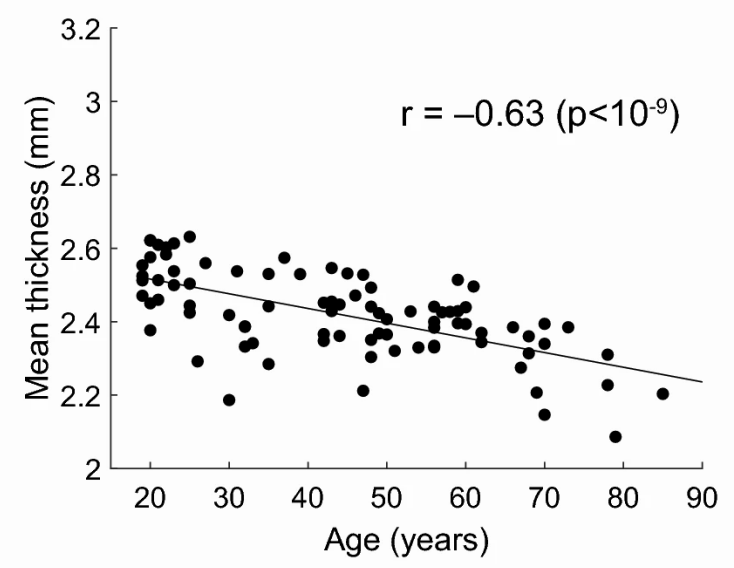

d

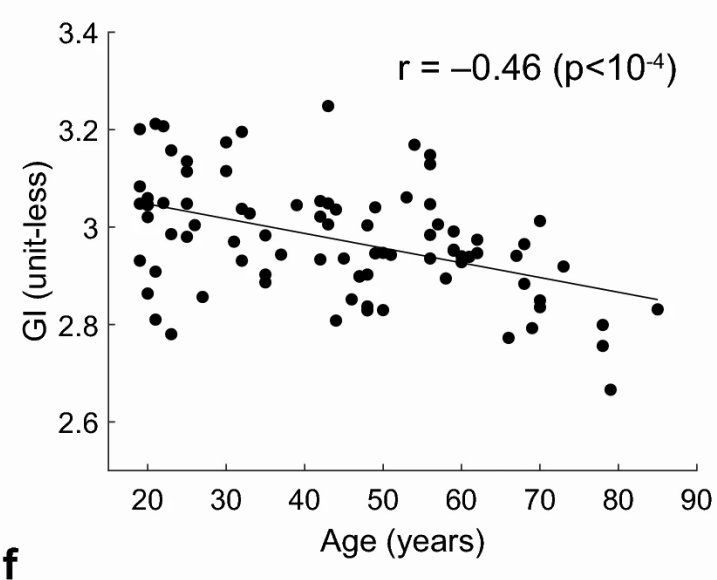

Source: () (MARZI et al., 2020). 


\section{Methodology}

\subsection{D images analysis using 1D transformations}

We designed the simplest experiment to help understand how the metrics change concerning the transformation, mainly because this was our first approach to MI metrics and registration.

The equations used to calculate the MI and entropies were copied from the literature:

$$
\begin{aligned}
H(x) & =\sum_{x} p(x) \times \ln p(x) \\
H(x, y) & =\sum_{x, y} p(x) \times p(x \mid y) \times \ln p(x \mid y) \\
H_{q}(x) & =\frac{1-\sum_{x} p(x)^{q}}{1-q} \\
H_{q}(x, y) & =\sum(p(x) * p(y))^{q} \times \ln _{q} p(x \mid y) \\
\ln _{q} x & =\frac{x^{1-q}-1}{1-q} \\
I_{q}(x, y) & =H_{q}(x)+H_{q}(y)-H_{q}(x, y)+(1-q) H_{q}(x) H_{q}(y)
\end{aligned}
$$

Figure 20 - BrainWeb Slice number 100

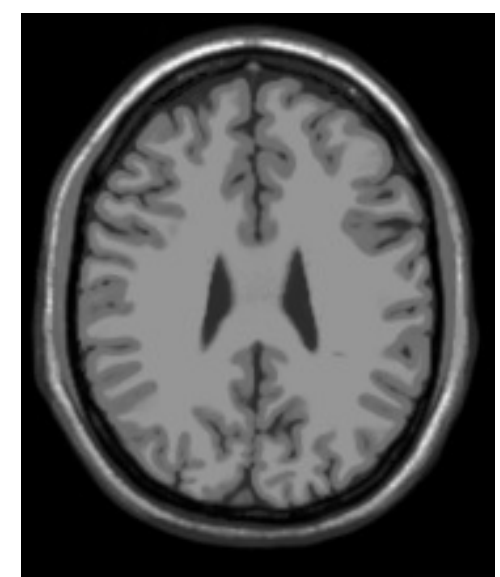

2D slice used for 1D transformation tests. Source: BrainWeb: Simulated Brain Database.

We used BrainWeb ${ }^{1}$ simulated MRI images in this test (KWAN; EVANS; PIKE, 1996; KWAN; EVANS; PIKE, 1999) due to the simplicity to achieve the images and the

1 Available at <https://brainweb.bic.mni.mcgill.ca/> 
quality provided as this image do not have any artifact or noise. It was selected, by visual inspection, a single slice of the MRI with a resolution of $181 \times 217$ pixels, shown in fig. 20 .

The main objective of this analysis is to understand MI metrics and how to interface with the ITK library to access image data, transform it and compare results.

Only translations were made on the $X$ and $Y$ axes, separately, over the range of $[-200,200] \mathrm{mm}$ in each axis.

Results are in sec. 4.1.

\subsection{D images analysis using 2D transformations}

Evolving the prior experiment (sec. 3.1) we start experiments with full 3D MRI images analyzing a 2D geometric transformation of translations and rotations. This experiment used the MI eq. 1.36, as the former experiment, but we calculated the joint entropy directly:

$$
\begin{aligned}
H(x) & =\sum_{x} p(x) \times \ln p(x) \\
H(x, y) & =\sum_{x, y} p(x, y) \times \ln p(x \mid y) \\
H_{q}(x) & =\frac{1-\sum_{x} p(x)^{q}}{1-q} \\
H_{q}(x, y) & =\frac{1-\sum_{x, y} p(x, y)^{q}}{1-q} \\
I_{q}(x, y) & =H_{q}(x)+H_{q}(y)-H_{q}(x, y)+(1-q) H_{q}(x) H_{q}(y)
\end{aligned}
$$

The images selected for these experiments were the N2_T1, N2_T2, and N4_T1, from the NAMIC Registration Library Case $19^{2}$, provided by the UNC Midas Database of healthy volunteers, with a size of $[176,256,176]$ and an isotropic spacing of $1 \mathrm{~mm}$. Those images belong to a tutorial on image registration using 3D Slicer. The images were considered a good basis for comparing the ITK Mattes used in 3D Slicer and our Shannon and Tsallis metrics implementation.

All probabilities from the transformation were precalculated and stored in an SQL database. Later this data was processed to calculate the entropies, and the final data was exported in a TSV/CSV format.

The translation was analyzed on the $X$ and $Y$ axes, jointly, over the range of $[-150,150] \mathrm{mm}$ and rotations on the $X$ and $Y$ axes, over the range of $\left[-180^{\circ}, 180^{\circ}\right]$.

Results are in sec. 4.2 . 


\subsection{D images analysis using 3D transformations}

The final analysis experiments are full 3D MRI images analyzed with the 3D geometric transformation of all affine components: translation, rotation, skewness, and scaling.

In these experiments we decided to test both MI equations, eq. 1.4 and eq. 1.36. The equations are the same as sec. 3.2, except for eq. 1.4 addition.

We used images from two databases, the Human Connectome Project (HCP) young adults (GLASSER et al., 2013; Van Essen et al., 2011), and the NAMIC Registration Library used in sec. 3.2. One can find the detailed description and the acquisition protocols at the HCP website $^{3}$. We selected only the MRI $3 \mathrm{~T}$ preprocessed structural images from the HCP data, initially using only the randomly chosen patients 172635 and 211821, and later using the entire HCP dataset for the Monte Carlo experiment (Sec. 3.4). The HCP dataset images have a size of $[256,320,320]$ and an isotropic spacing of $0.7 \mathrm{~mm}$.

Histogram binning is implemented by disabling the least significant bits of the images, in a similar way done by (COLLIGNON et al., 1995b). All images voxels were converted to 16 bits unsigned integers (uint16_t), and then applied a simple bitwise AND operator to the voxels and the binning bitmask. The notation used is for the number of bits left in the image, so when we use 6 bits binning histogram, we leave only 6 bits of information on both images and use a bitmask of 1111.1100.0000.0000 on the images. As a result, the joint histogram will have double bits than single images, i.e., $2 \times 6=12$ bits, resulting in 4,096 gray levels. We also first applied a normalization to spread the histogram to all the 16 bits levels, in order to improve the binning results: $x^{\prime}=2^{16} \times\left(x-x_{\min }\right) /\left(x_{\max }-x_{\min }\right)$, where $x^{\prime}$ is the normalized gray value, $x$ is the input value and $x_{\max }, x_{\min }$ are the maximum and minimum values in the entire image.

\subsubsection{Metric image visualization}

To understand the metrics reaction to geometric transforms we mapped sets of transform parameters into a 3D output space, with different mappings depending on the transformation, as follows:

Translations are mapped directly to a cube with a range of $[-150,150] \mathrm{mm}$ in all axes.

Rotations are mapped directly to a cube with a range of $[-1,1]$ in the case of versors, and $\left[-180^{\circ}, 180^{\circ}\right]$ in the case of degrees.

$3<$ http://protocols.humanconnectome.org/HCP/3T/imaging-protocols.html> 
Scalings are mapped to a cube with the mapping function:

$$
\begin{array}{ll}
s=\frac{1}{(1+x)} & \text { if } x<0 \\
s=1 & \text { if } x=0 \\
s=(1+x) & \text { if } x>0
\end{array}
$$

where $s$ is the real scaling and $x$ is the cube coordinate. This will map scalings in a range of $[0.5,2]$ to the cube $[-1,1]$ range.

Skewness are mapped directly to a cube with $[-1,1]$ range in the three axes.

This cube space was discretized using 51 points in each direction, i.e., a dimension of $51 \times 51 \times 51$.

Each voxel of this cube represents a single unique transformation parameter $T$, e.g., a translation of $[6,12,24] \mathrm{mm}$ in the axes $x, y$ and $z$, respectively, would be mapped to point $[26,27,29]$, and the point $[25,25,25]$ would be the center, i.e., $[0,0,0] \mathrm{mm}$ translation.

After defining the cube dimensions, and the transformation parameters $T$ for all cube voxels, we populate this cube with the metric signal for each parameter $T$, allowing us to inspect this cube information and compare the multiple metrics signals, i.e., comparing Mattes, Shannon, and Tsallis.

This 3D cube, with the metric's outputs, was saved into a VTI file, from VTK library, with each $q$ parameter having a separated dataset inside the image, sharing only the structured grid, i.e., spacing and the dimensions sizes. In this way, we have multiple 3D cube images, one for each $q$ parameter, in a single image file.

\subsubsection{ParaView Analysis}

ParaView is an open-source, multi-platform data analysis and visual-
ization application. ParaView users can quickly build visualizations to
analyze their data using qualitative and quantitative techniques. The
data exploration can be done interactively in 3D or programmatically
using ParaView's batch processing capabilities. ParaView was developed
to analyze extremely large datasets using distributed memory computing
resources. It can be run on supercomputers to analyze datasets of petas-
cale as well as on laptops for smaller data. ParaView is an application
framework as well as a turn-key application. (OVERVIEW...,)

The image registration process is based on the gradient descent, or steepest descent, algorithm. The optimizer starts in an unknown coordinate and travels around the steepest descent path to find a local minimum. This travel is done by evaluating the gradient of the function being optimized and following the way as opposed to the gradient. 
The gradient of a function points to the way that maximizes its growth. However, in our reality, the most common need is to minimize a function, e.g., minimize the cost or time needed to do a task, so most optimization algorithms are built to find the minimum point. That is the reason why we follow the way as opposed to the gradient.

ParaView possess a built-in gradient filter, named Gradient, that applied to the MI data yields our $\frac{\partial F_{m}}{\partial T}$ as a gradient vector field. The StreamTracer filter will plot this field joining vectors in lines. The most common usage of this filter is to plot the airflow in aerodynamics. However, any vector field can be visualized by this tool.

Another filter also used to visualize the metric space is the Contour field, which will create isosurfaces on an image where the function is equal to a certain level. The gradient is normal to isosurfaces, i.e., perpendicular, so the more spherical a surface is around a point, the more the gradient will directly point to it.

Fig. 21 shows examples, from our result section, of images generated by those filters. In this case, the function being analyzed is the Shannon metric concerning the translation transformation. The formal equation for our gradient is on eq. 3.1:

$$
\nabla F_{m}=\frac{\partial F_{m}}{\partial T_{x}} \hat{\mathbf{i}}+\frac{\partial F_{m}}{\partial T_{y}} \hat{\mathbf{j}}+\frac{\partial F_{m}}{\partial T_{z}} \hat{\mathbf{k}}
$$

Where $\nabla F_{m}$ is the gradient, composed by a partial derivative in each of the directions $\hat{\mathbf{i}}, \hat{\mathbf{j}}$, and $\hat{\mathbf{k}}, T_{x}$ is the $x$, or $\hat{\mathbf{i}}$ component of our translation parameter $T, T_{y}$ and $T_{z}$ are defined similar to $T_{x}$.

ParaView results are in sec. 4.3 .

\subsubsection{Naive registration capacity}

The registration capacity of the metric, under a gradient descent, or ascent, algorithm, can be naively deduced by a simple algorithm, represented in alg. 1. We start with our center point $I_{c}$ as a seed. For all neighbor voxels of our seeds, we test if they have a smaller value than the seed. If true, we add them to the seed set for the next iteration. Thus, all seeds are saved in our volume set $V$ as registrable voxels. To simulate a gradient ascent, the same algorithm is also done in the opposite direction by adding to the seed only neighbors with greater value than the seed in question.

The ratio between the number of voxels in the metric space and the number of registrable voxels gives an approximation of the registration capacity of the metric.

All registration capacity results are in sec. 4.4. 
Figure 21 - Example of the ParaView filters

(a) StreamTracer

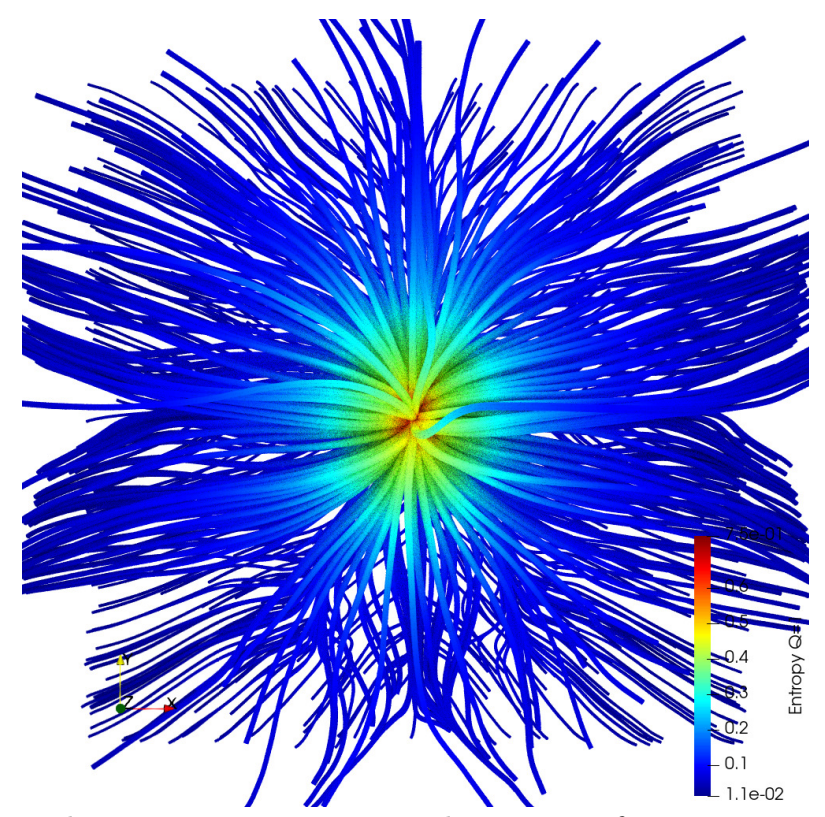

(b) Contour

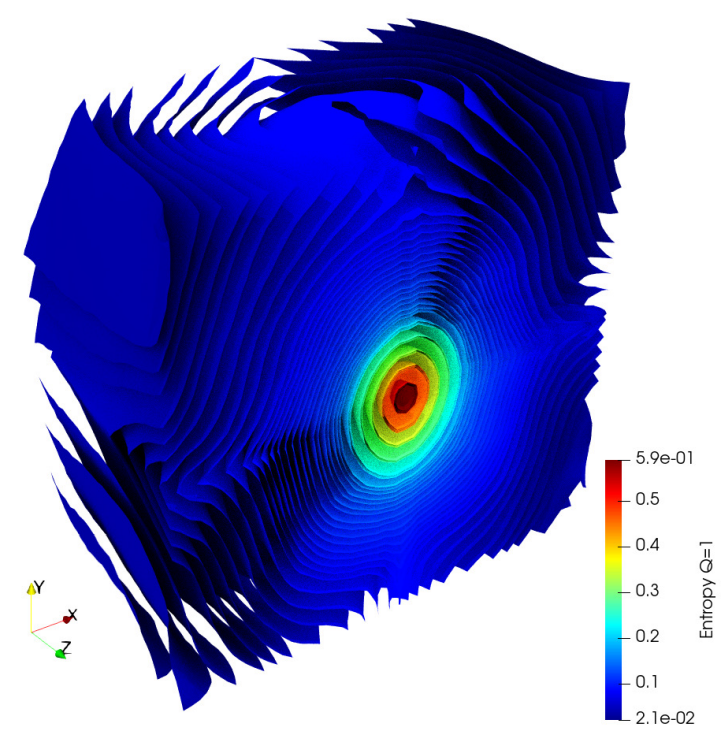

Filters StreamTracer and Contour from ParaView on Shannon metric in relation to translations. Source: author.

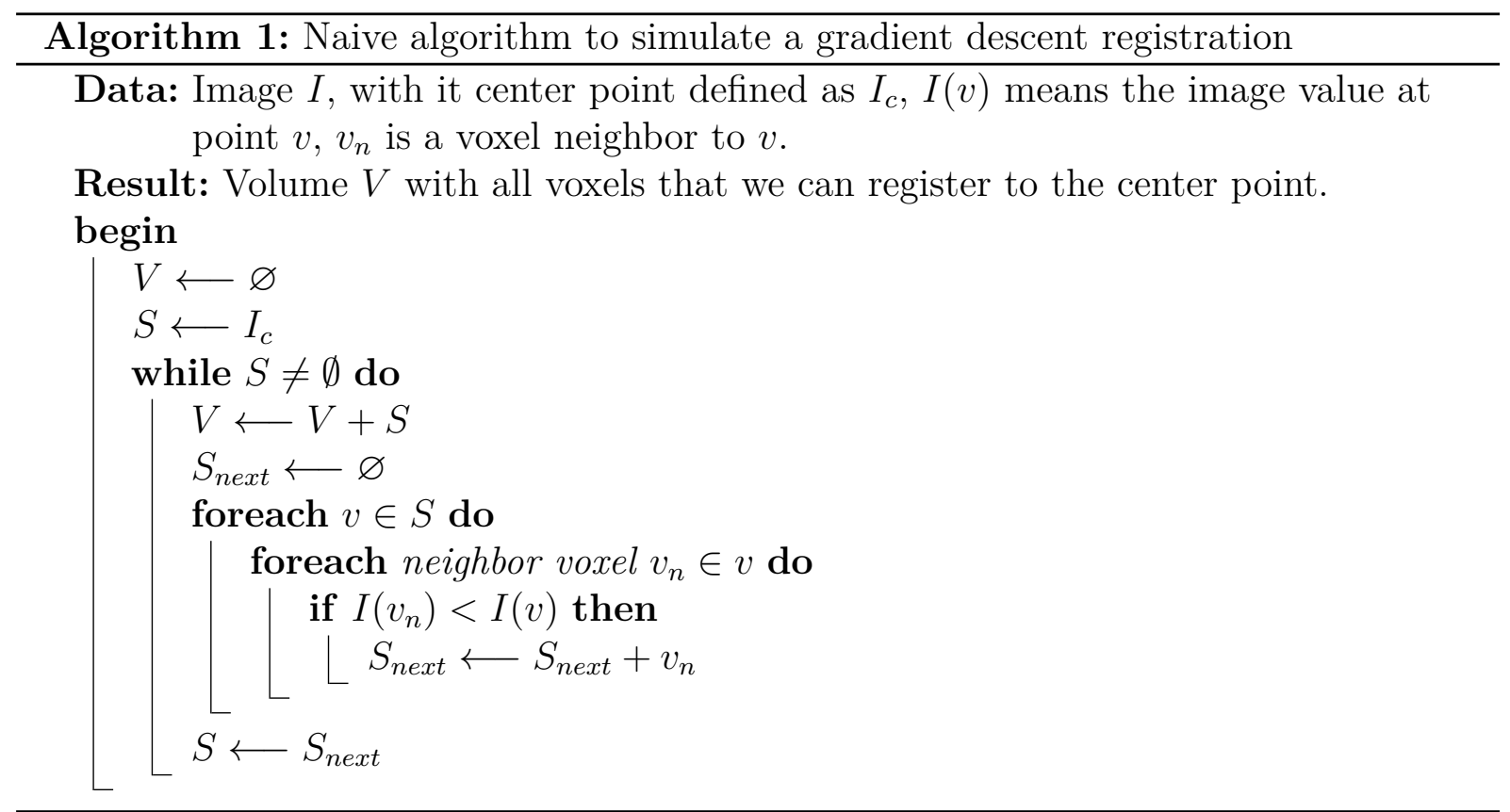


Table 1 - Registration scenarios using Monte Carlo simulation.

\begin{tabular}{lccl}
\hline Name & Fixed & Moving & Subject \\
\hline T1 & T1 & T1 & Same \\
T2 & T1 & T2 & Same \\
Randomized T1 & T1 & T1 & Randomized \\
Randomized T2 & T1 & T2 & Randomized \\
\hline
\end{tabular}

Source: author.

\subsection{Monte Carlo Simulation}

To further evaluate the metric performance on the translation transform, a series of Monte Carlo simulations were carried out by probing translation parameters using a normal distribution with a standard deviation of $50 \mathrm{~mm}$, giving a three-sigma region (99.7\% of the transforms) within $[-150 \mathrm{~mm}, 150 \mathrm{~mm}]$, in each one of the translation axes.

We simulated four scenarios for the image registrations, enumerated in table 1 , with the first two scenarios using a single subject image and the latter two scenarios using a set of double randomized subjects from the HCP dataset.

These simulations provide additional reliable assessments than the ones from sec. 3.3.3 as the randomized scenarios simulate inter-subject and inter-modality clinical registrations. An essay consists of 1,000 (or more) registration trials, logging each registration start parameters, end parameters, fixed and moving images used, the time spent registering, and a flag if the registration is within $5 \mathrm{~mm}$ of the center. Since the HCP images are rigidly registered, we used the center as a gold standard.

The simulations marked with the subject as "Same" have single randomization made on each iteration, drawing a subject from the list and using this subject to both fixed and moving images. The simulations with a "Randomized" subject have each fixed and moving image fully randomized. In this way, the same subject experiment deals with the metric capacity of registering an inter-subject registration, with intra-modality (T1 vs. T1) or inter-modality (T1 vs. T2). The randomized subject deals with the metric capacity of inter-subject registration.

Results are in sec. 4.5 . 



\section{Results and Discussion}

To better understand the properties of generalized entropies, like Tsallis' entropy, we will analyze the results collected over our research in this chapter. We initially used only the nonadditive equation since it was used in the academy but with no promising results. Later on, the additive equation, as initially developed by Shannon, is used, achieving better results on translation. Finally, by using the 3D isosurfaces, we better understand the metric space. However, neither the additive nor nonadditive versions of mutual information can, by themselves, solve the registration problem. To achieve the best metric space and registration possible, we need to use an ensemble of multiple metrics to combine both additive and nonadditive and histogram binning in rotation.

The initial results were preserved in this thesis to show the evolution of the research in the most transparent way possible, noting that they should not be followed to develop a metric algorithm as the later experiments and equations used provides a way better overall result.

\subsection{D images with 1D analysis (2017)}

The results in this section follow the definitions on sec. 3.1.

In all figures (fig. 22 and fig. 23) we have the Mattes' entropy plotted as a dashed black line, the colored solid lines are all Tsallis' entropy with only a different $q$ per line.

Those results are 1D plots, where the variable is the translation offset, in the $x$ or $y$ axis as noted, of an MRI 2D slice.

Fig. 22 illustrates the translation on $x$ axis, where fig. $22 \mathrm{a}$ is for $q \leq 1$ with translations in the range $[-200,200] \mathrm{mm}$. The Mattes capture region was approximated to $[-27,29] \mathrm{mm}$ and is shown in the red-shaded region. Fig. $22 \mathrm{~b}$ shows a zoom in the range $[-35,35] \mathrm{mm}$, where the details show a much wider capture range for Mattes in the red-shaded region, and the capture ranges for Tsallis is narrow. The only advantage for Tsallis here is the steeper gradient towards the center. For the $q \geq 1$ scenario (fig. 22(c-d)) the Tsallis capture range becomes too narrow and is not useful.

Possible use of Tsallis, as though at the time, was to use Tsallis in an inverted way, looking for its minimum, as in $q \geq 1$ we have a capture range wider than Mattes. However, this only works as a first stage registration as the minimum points are offset from the center, as can be seen on fig. $22 \mathrm{~d}$ as a yellow curve.

Translating on the $y$ axis (Fig. 23) gives a similar result to the $x$ axis, noting now 
Figure 22 - MI results from 2017 with translations on the $x$ axis

(a)

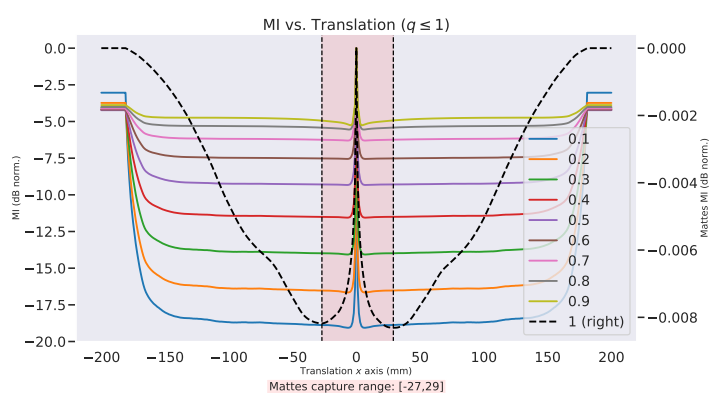

(c)

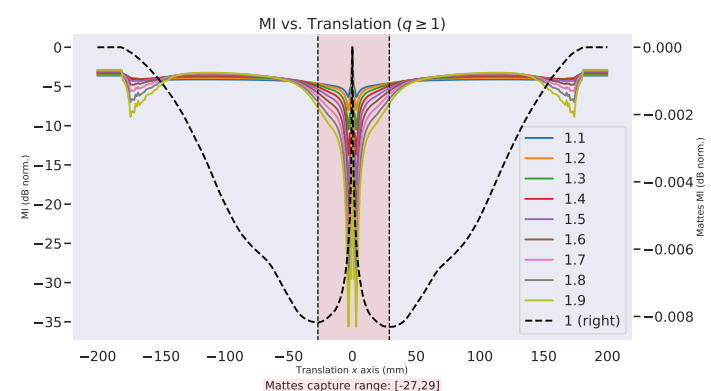

(b)

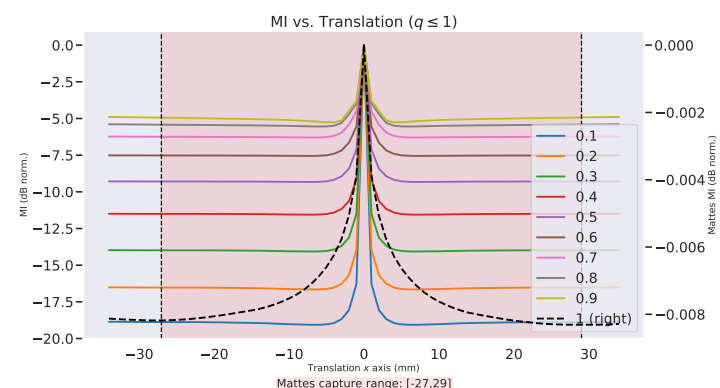

(d)

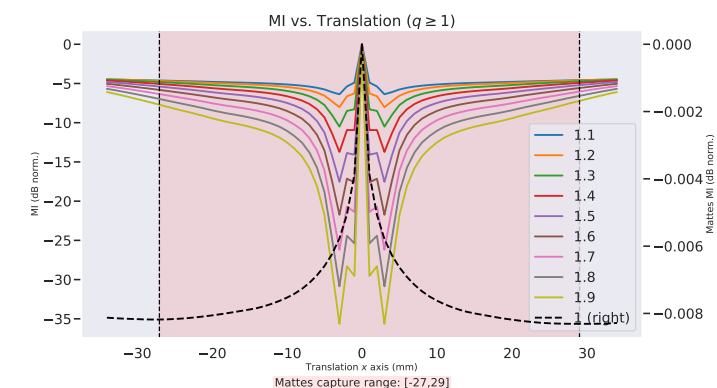

MI results from early research in 2017 with translations on the $x$ axis, Mattes metric dashed black with capture range in red-shaded region: (a) $q \leq 1$ (b) $q \leq 1$ zoom (c) $q \geq 1$ (d) $q \geq 1$ zoom. Source: author.

a more asymmetrical shape for the Mattes metric, the black dashed line on figs. 22(a,c), the capture regions were approximated to $[-31,34] \mathrm{mm}$ and were wider than the Tsallis regions.

The shape of each metric curve can be better compared, in a qualitative way, as shown in fig. 24 and fig. 25. The Tsallis MI seems to "distort" the Mattes metric (our $q=1$ metric) by either expanding the capture range $(q<1)$ or narrowing it $(q>1)$. The main problem is that the expansion on $q<1$ does not provide a good gradient. It has a very steep gradient around the center, but the gradient diminishes and changes the signal closing the capture range around it.

As an advantage, Tsallis have a symmetrical shape on $y$ axis translations, where Mattes shows the asymmetrical shape. This can be better seen in fig. 23 and fig. 25 . 
Figure 23 - MI results from 2017 with translations on $y$ axis

(a)

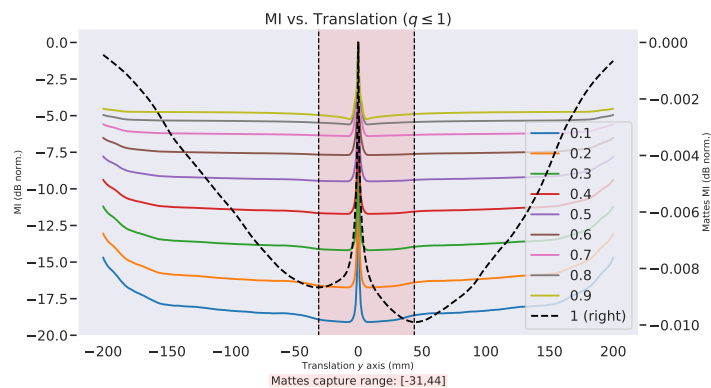

(c)

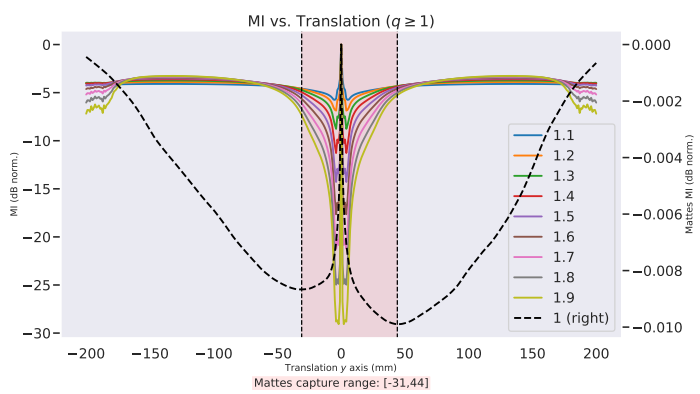

(b)

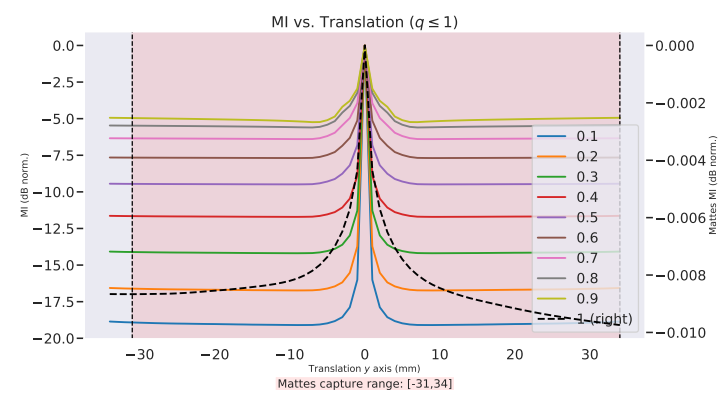

(d)

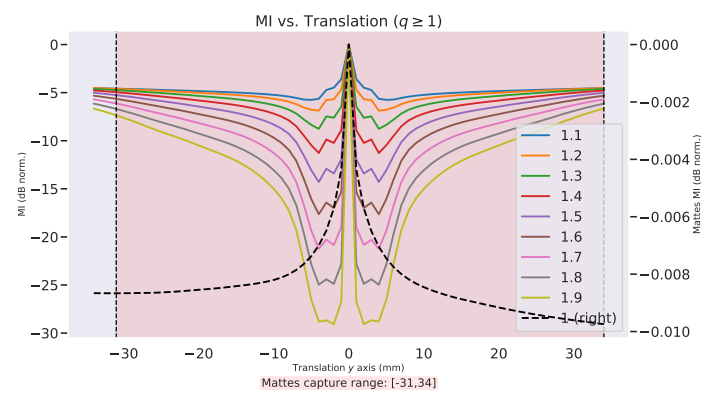

MI results from early research in 2017 with translations on $y$ axis, Mattes metric dashed black with capture range in the red-shaded region: (a) $q \leq 1$ (b) $q \leq 1$ zoom (c) $q \geq 1$ (d) $q \geq 1$ zoom. Source: author. 
Figure 24 - MI vs. Translation on $x$ axis

\section{MI vs. Translation on $x$ axis}
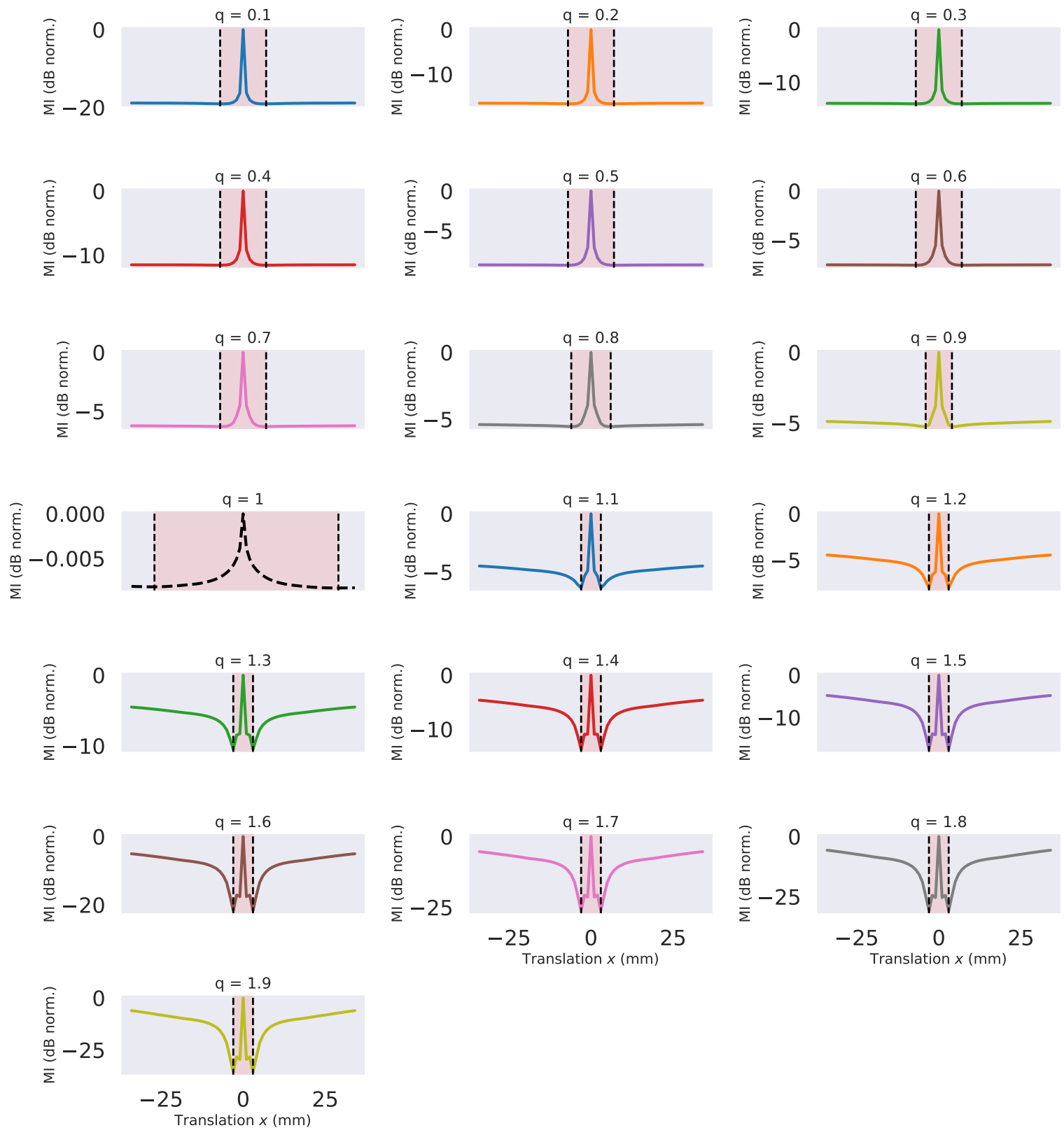

MI vs. Translation on $x$ axis, to compare metrics output curves qualitatively. Same plots from fig. 22, but each line separately. Source: author. 
Figure 25 - MI vs. Translation on $y$ axis

\section{MI vs. Translation on $y$ axis}
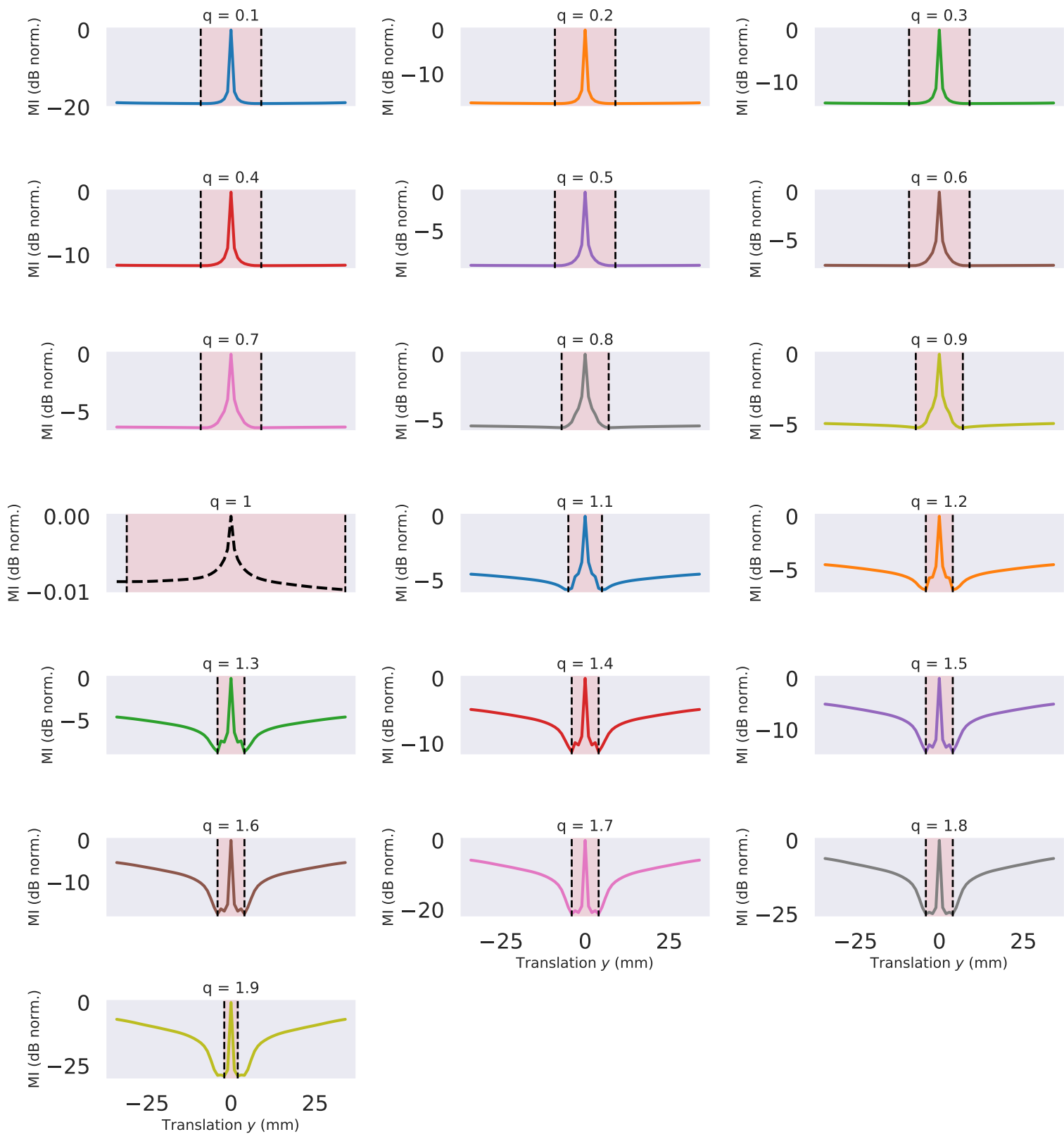

MI vs. Translation on $y$ axis, to compare metrics output curves qualitatively. Same plots from fig. 23, but each line separately. Source: author. 


\subsection{D analysis with 2D translations and rotations}

\subsubsection{Translation}

We start analyzing how our Tsallis metric changes very close to $q \rightarrow 1$, fig. $26 \mathrm{~d}$ shows the Mattes Metric $(q=1)$ and some extrema on the corners. Our capture range is contained in the central region. Those extrema will catch long-range registration.

If we go $q<1$, the extrema grows (fig. 26a-c), diminishing our capture range. As in the $1 \mathrm{D}$ analysis case, there is some argument if in the central region the metric gives a better gradient or not, but the fact remains that our capture range is narrow when $q<1$ in this experiment.

For the $q>1$ case, if we raise $q$ a bit over Shannon we have good results, as shown in fig. 26e-f for $1.04>q>1.15$, where we have an approximately full capture range, but as we go near the range's end we can see the gradient paths not pointing directly to the center (fig. 26e), but pointing to the diagonals were the extrema appears on Shannon. With just a little adjustment, going from $q=1.15$ to $q=1.2$ (fig. 26f-g) our extrema dettach from the center and any registrations near them will get caught, resulting in a very narrow capture range for $q=1.2$.

Raising the $q$ parameter on the $q>1$ range seems to create new extrema from the center that will eventually merge with the corners extrema and create a single capture range. As can be seen from the sequence in fig. 26(e-h). In the limit where $q \rightarrow 1$ we can think of the extrema converging to the center and moving away as $q>1$. The $q<1$ scenario provides a different effect, where the extrema from the corner grow as we lower $q$. Eventually, the extrema's region grows, but the center will remain, giving us a pseudocapture range, where we can use it to register an image. However, we will not get a perfect registration, only a near approximation. 
Figure 26 - MI function changes with 2D translation of an 3D image

(a)

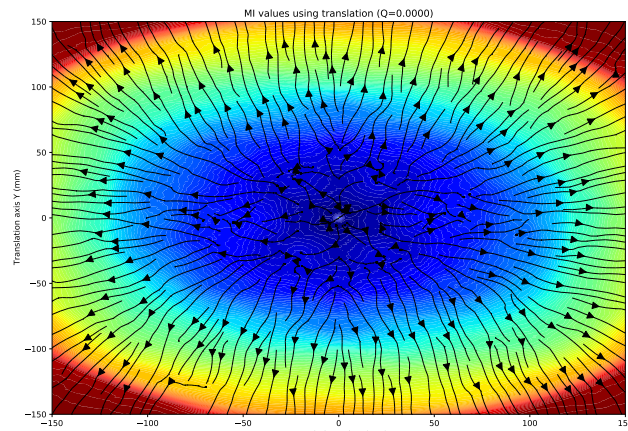

(c)

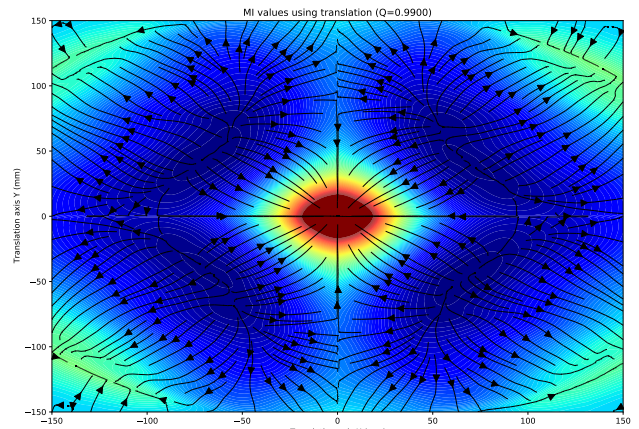

(e)

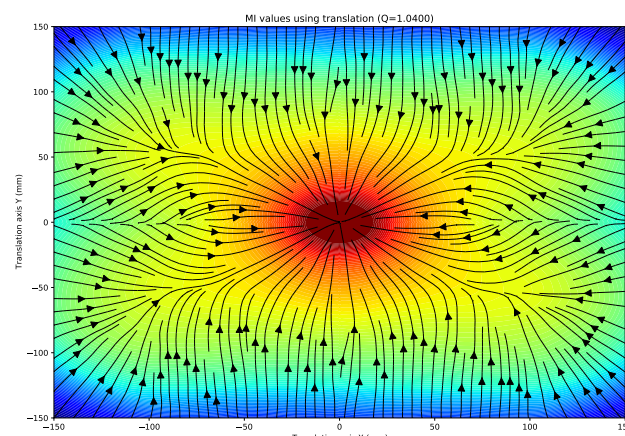

(g)

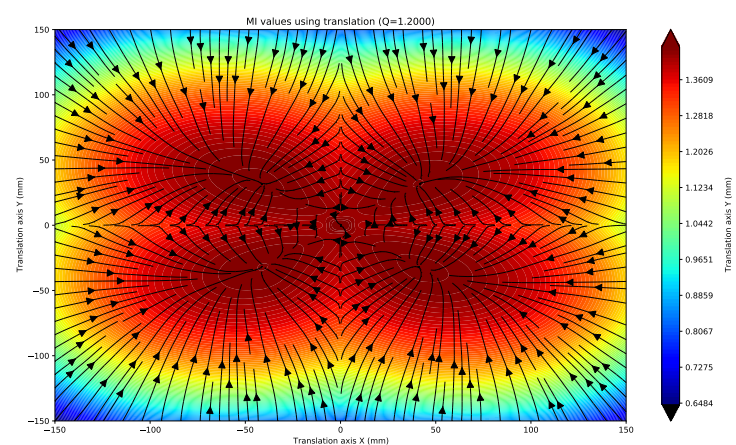

(b)

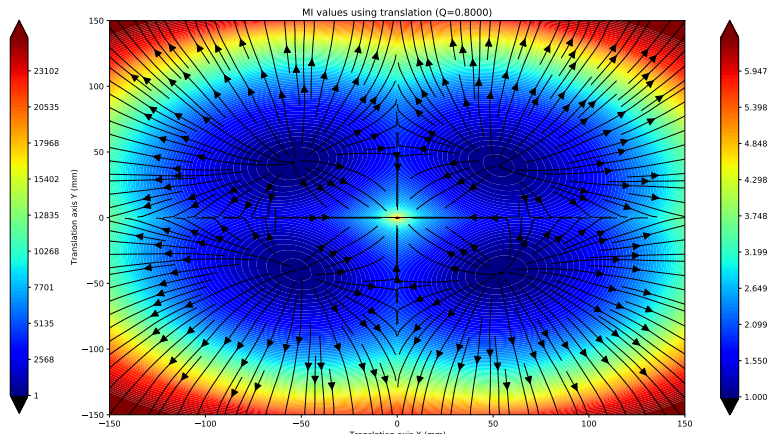

(d)

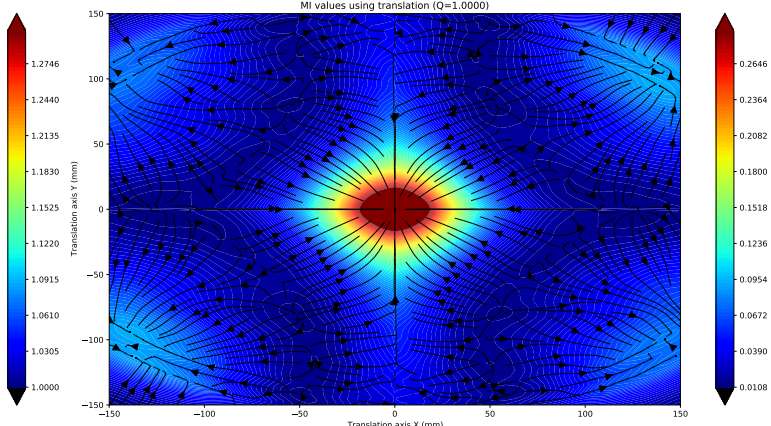

(f)

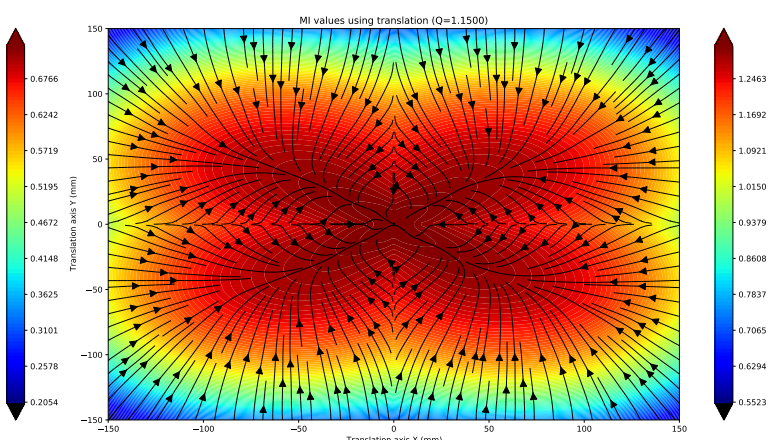

(h)

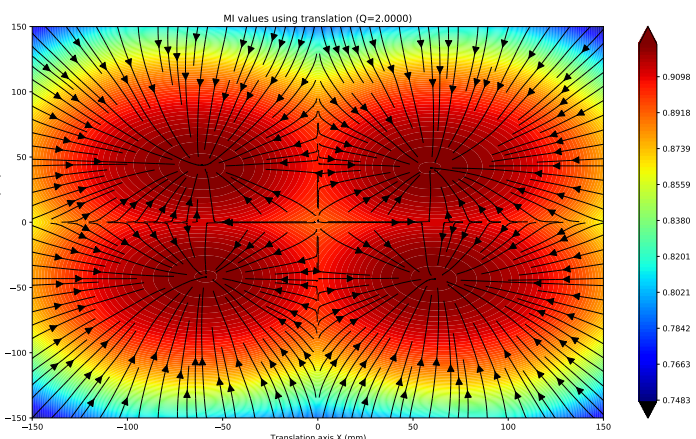

MI function changes with 2D translation of an 3D image: (a) $q=0$ (b) $q=0.8$ (c) $q=0.99$ (d) $q=1$ (e) $q=1.04$ (f) $q=1.15$ (g) $q=1.2$ (h) $q=2$. Source: author. 


\subsubsection{Rotation}

Rotation analysis showed similar results to translation, fig. 27d is the Mattes metric output with a big extremum in the northeast corner (dark red) and a small extremum in the southwest corner (light blue region). Also, other minor extrema appear, and the capture range is very limited in this scenario. It also should be noted that we have an unstable gradient field on the northwest and southeast corners of the space.

Raising to $q=1.01$ (Fig. 27e), using Tsallis entropy, but very close to the Shannon limit $(q \rightarrow 1)$, the regions on northwest and southeast corners gain some stability. Of course, the two extrema from Shannon still exists, but overall, we have a wider capture range, mainly from the stability gain. Raising more to $q=1.1$ (Fig. 27f), the extremum on the southwest region seems to merge with the center. Also, the northeast extremum still troubles registration. The main gain is on the Shannon unstable regions that are now very stable and provide a wider capture range. Raising more to $q=1.5$ (Fig. 27g), we have our Shannon extrema with two new extrema on the northwest and southeast region. Now all four quadrants of the figure have extrema and dominate the registration, narrowing our capture range. This effect seems to become worst for $q=2$ (Fig. 27h) and greater values.

Lowering the entropic index to $q=0.99$ (fig. 27c) changes the unstable regions into more stable regions but still converges to new extrema points making this stability not useful for our purposes. With $q=0.97$ (fig. 27b), we see a more stable gradient field with the southwest extremum moving closer to the center, narrowing our capture range, as for the translation when $q<1$, we start to see that the gradients are pointing outwards. In the extreme range in our experiment, $q=0.1$ (fig. 27a), we have a very narrow capture range. We can see the center light blue, meaning it has greater entropy than the surrounding space. However, the gradient field changes its signals near the center, which is very similar to the translation scenario. 
Figure 27 - MI function changes with 2D rotation of an 3D image

(a)

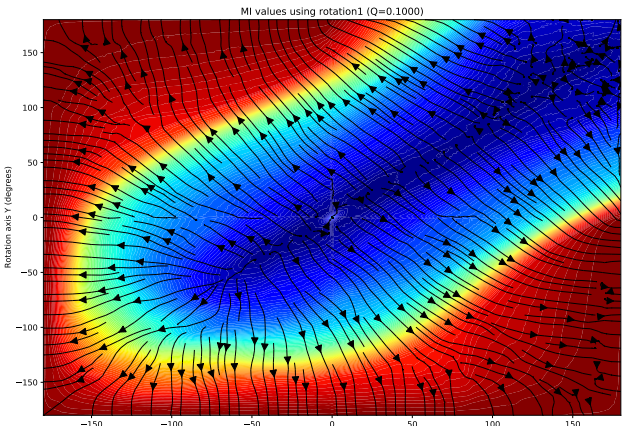

(c)

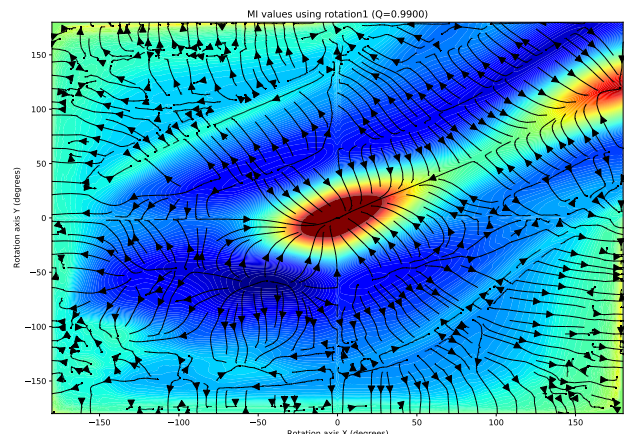

(e)

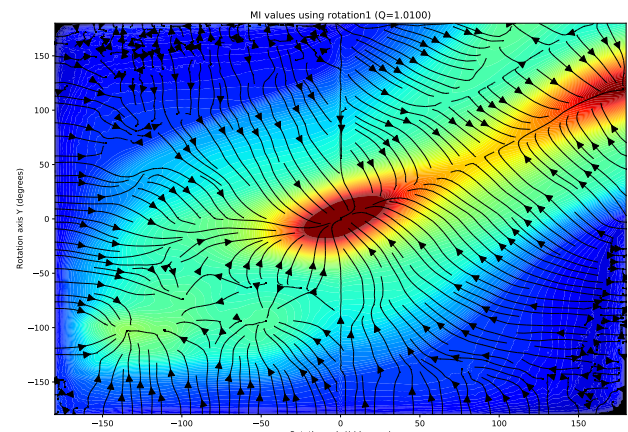

(g)

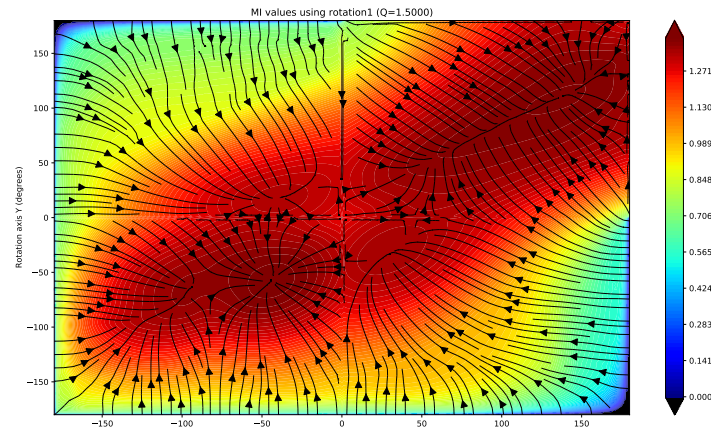

(b)

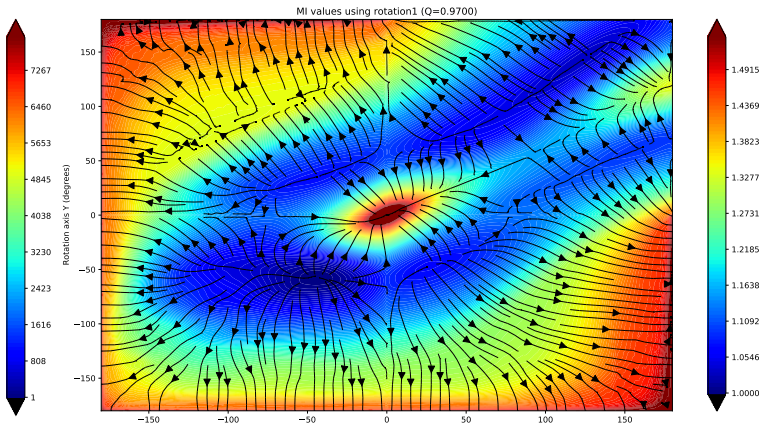

(d)

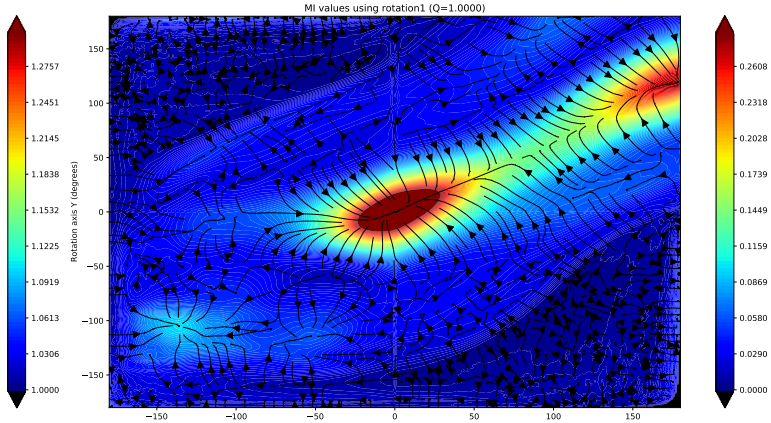

(f)

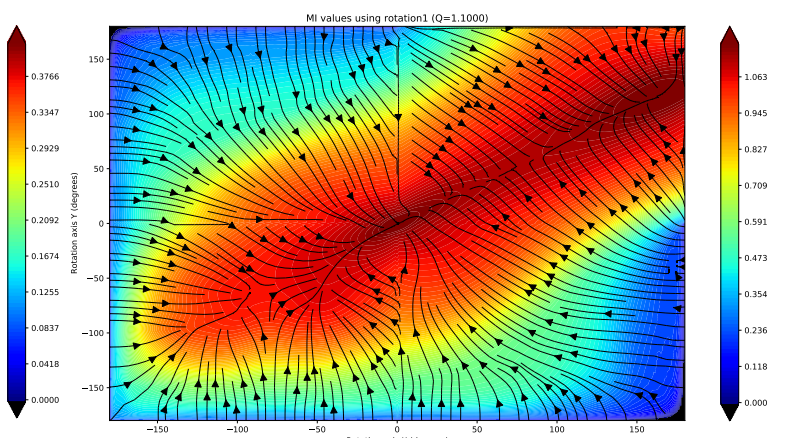

(h)

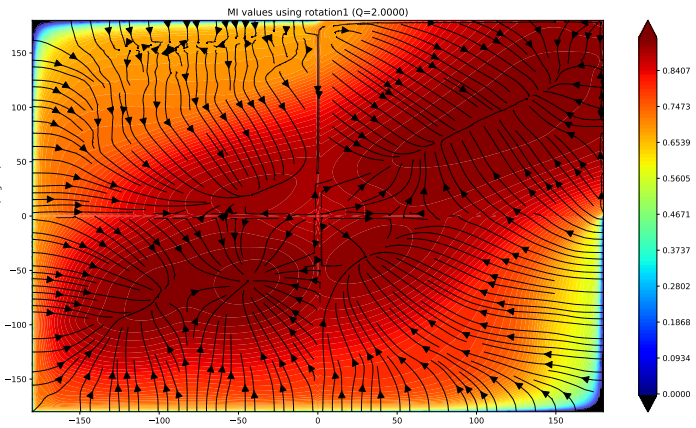

MI function changes with 2D rotation of an 3D image: (a) $q=0.1$ (b) $q=0.97$ (c) $q=0.99$ (d) $q=1$ (e) $q=1.1$ (f) $q=1.5$ (g) $q=2$. Source: author. 


\subsection{D images with 3D analysis (2019)}

\subsubsection{Introduction}

This section introduces the analysis of 3D metric spaces, studying how the metric changes on a specific transformation, e.g., translation or rotation, in all possible axis. This $3 \mathrm{D}$ analysis is a powerful tool since it shows the details of the metric space that other tools analyzing $1 \mathrm{D}$ or $2 \mathrm{D}$ spaces cannot explore.

For a better argument on the benefits of a complete 3D analysis, we created fig. 28, where (a) shows our proposed analysis tool with MI isosurfaces. Those isosurfaces are regions of our metric space where the MI has the same value. Thus, they are similar to $2 \mathrm{D}$ contour curves are used in topography to detail the altitude changes in a $2 \mathrm{D}$ space.

The 3D image shown in fig. 28(a) can provide much denser information on the metric. To understand this density, let us use the subfigure (a) to extract some 1D plots, like those used in sec. 4.1 and most papers in the literature. Fig. 28(b) shows the same image as (a) with more transparency. This transparency allows us to better see the minimum points inside the space, which will attract the registration optimizer. On (b), some cylindrical structures signal paths, i.e., lines, from where we extracted data to create subfigures (c-d). Those paths are along the main axes and the principal diagonals, with one on the diagonal where $z=0$, from $(-150,150,0) \mathrm{mm}$ to $(150,150,0) \mathrm{mm}$ and the other is the major diagonal, from $(-150,-150,-150) \mathrm{mm}$ to $(150,150,150) \mathrm{mm}$.

Fig. 28(c-d) shows the 1D plots from the cylindrical structures on (b), with the paths on main axes in dashed lines and the two diagonals in solid lines. This analysis shows an interesting behavior of ITK Mattes. The dashed lines along the axes are good registration paths, providing a broad capture range along those lines without local minimum. However, the diagonal paths have a narrow capture range, with some local minimum.

We hope this provides a solid argument for 3D analysis or at least using 2D analysis if $3 \mathrm{D}$ is impossible. Suppose we experiment only with 1D analysis and select only the axes lines. In that case, our experiment will show ITK Mattes as an excellent metric capable of registering all the space, a wrong statement, as we can see from (a). The line diagonals surely improve the 1D analysis, but it may be possible that our metric works on the axes and diagonals lines and have problems on other regions.

The problem with $1 \mathrm{D}$ analysis repeats itself with 2D analysis. We are only analyzing a fraction of our space. The complete 3D space analysis provides all possible translations, or rotations on the space, missing no local minima or maxima points that $1 \mathrm{D}$ and $2 \mathrm{D}$ analysis can miss.

The only drawback is that we can only analyze a 3D space, while our full affine metric has a $12 \mathrm{D}$ space: $3 \mathrm{D}$ for translation, 3D for rotations, 3D for scaling, and 3D 
for skewness. There seems no current way to overcome this "curse of dimensionality", as is called in literature, since a 3D analysis with 51 points in each dimension has $51 \times 51 \times 51=51^{3}=132,651$ points, while a $12 \mathrm{D}$ analysis with only 3 points in each dimension has $3^{12}=531,441$ points. The information from analyzing only 3 points in each dimension is inferior. Hopefully, we will be able to analyze a higher number of points in the future, and better visualization techniques will be implemented. For now, we are stuck on analyzing 3D spaces.

\subsubsection{Translation}

Fig. 30 compares the ITK Mattes and Shannon metrics. Fig. 30 (a-b) are the MI isosurfaces and Mattes implementation is very concentric around the center. However, as the distance from the center increases, the instability of the gradient field grows, new extrema emerge, and the surfaces become convoluted around those extrema. In comparison, Shannon in subfigure (b) shows smooth surfaces convoluted around the center.

The consequences of the extrema are shown in the gradient paths, shown in fig. 30 $(c-j)$, where the Mattes metric has gradient paths emerging from the bottom (c-d) to those extrema while Shannon has almost all bottom paths oriented to the center. Fig. 30 (g-h) provides a better understanding of the Mattes extrema, where $(\mathrm{g})$ is the view from the top $z$ axis and each corner has a pole with paths oriented to them, when we add the center paths to the image (shown in h), Mattes shows a narrow capture range, represented on fig. 30 (c-j) by the block of paths on the image center. In comparison to Mattes, Fig. 30 (i-j) shows the paths for Shannon where the center block covers all the space, fully connected, which translates to a wide capture range.

A comparison of Shannon and Tsallis, using the same eq. 1.4 for both metrics, is shown in fig. 31. In the MI isosurfaces, the most perceptible difference is the roundness of the surfaces: Shannon (a) has symmetry; however, the surfaces are far from spherical where Tsallis (b), with $q=1.5$, shows a more spherical shape, with more roundness.

For a better view of the MI metrics, we can use the gradient paths on fig. 31c-j where we can see, in the Shannon metric, that the bottom paths to the center $(c-d)$ do not follow a straight line. Instead, they show some turbulence in the MI space. On the other hand, in the Tsallis case (e-f), the bottom paths follow more direct lines to the center, near an ideal straight path. The top view of the metrics (g-j) also reflects this difference, with Shannon (g-h) showing turbulence and a tendency to group before reaching the center while Tsallis ( $i-j)$ show more direct lines that flow independently to the center.

Finally, we need to compare the Tsallis approach proposed here, using eq. 1.4, with the proposal on the literature, using eq. 1.36. The comparison is shown on fig. 29 with the Mattes (a), Shannon (b), Tsallis (c) as we described before, and the Tsallis non-additive 
Figure $28-3 \mathrm{D}$ analysis introduction

(a)

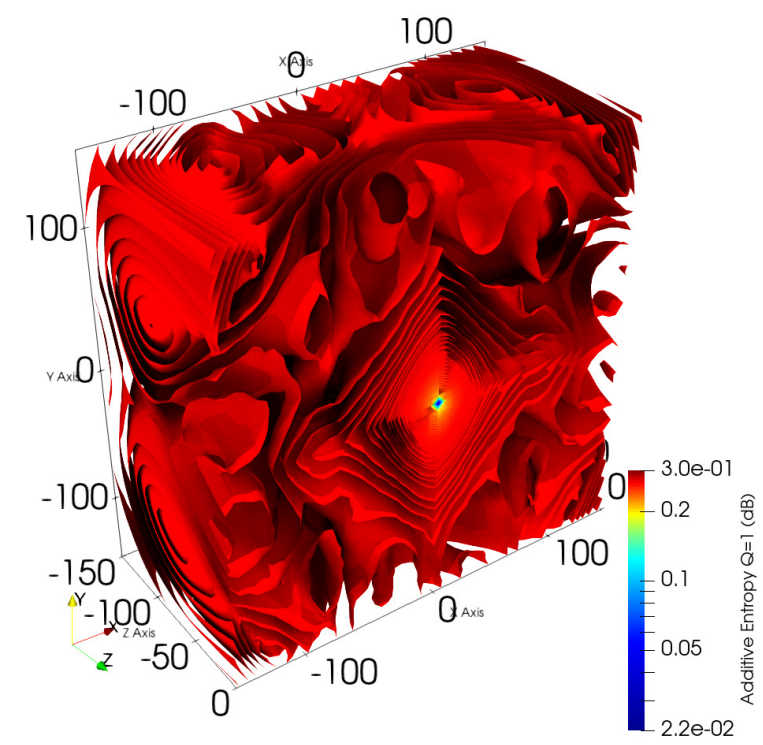

(c)

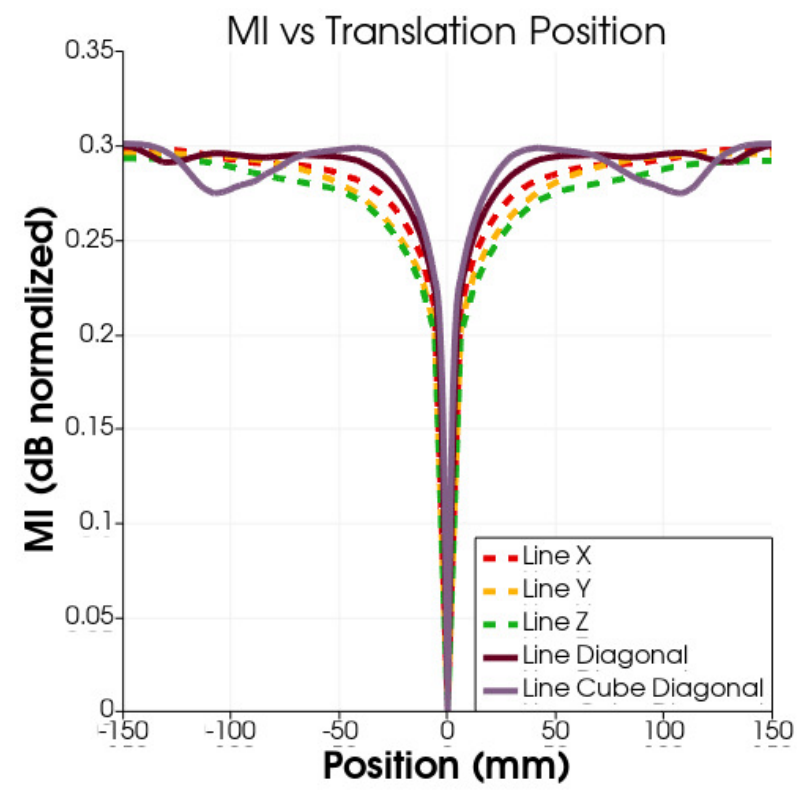

(b)

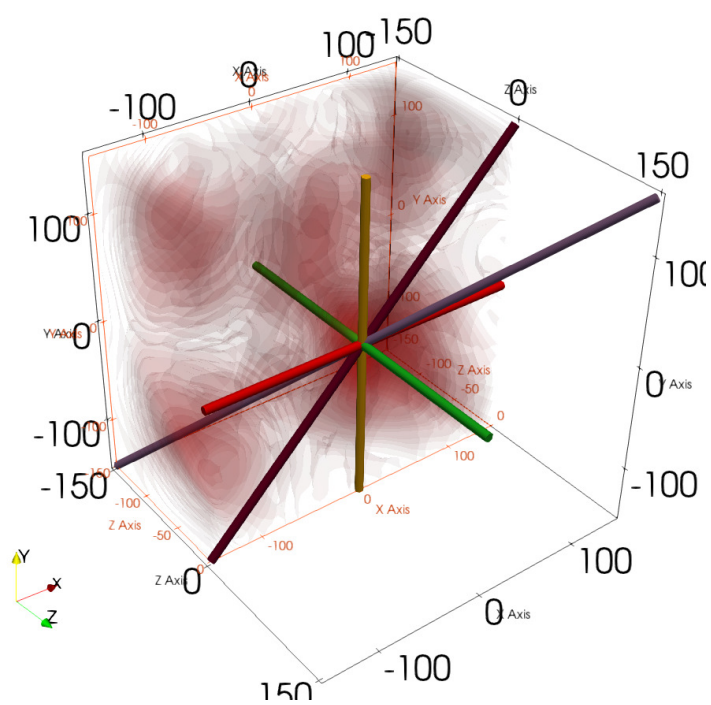

(d)

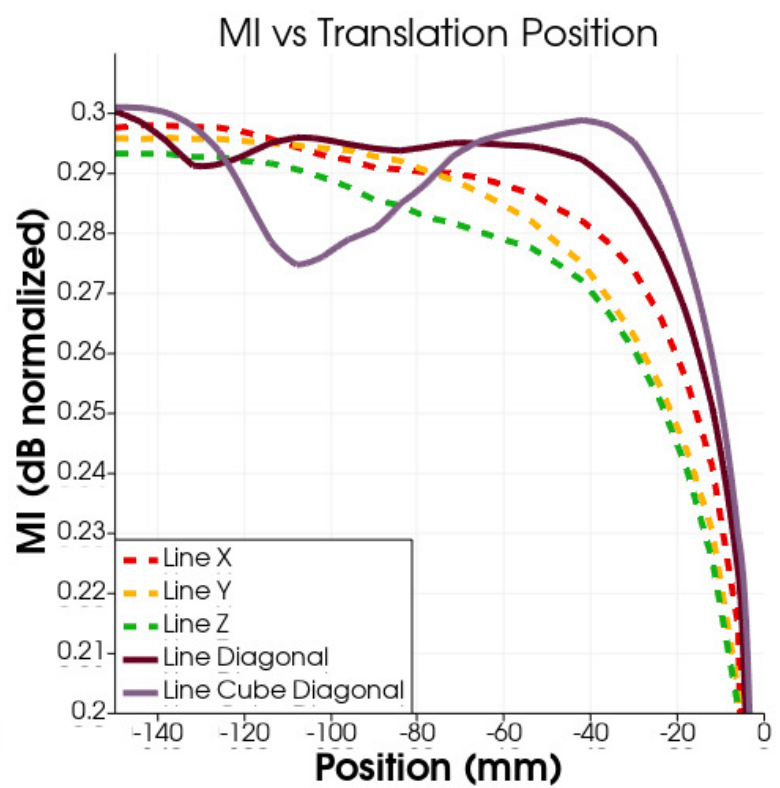

3D analysis introduction: (a) 3D isosurfaces for ITK Mattes (b) same with added transparency and line paths for the (c-d) 1D plots of MI values over a line. The colors of (b-d) are the same. Source: author. 
Figure 29 - Translation Metric's 3D isosurfaces

(a)

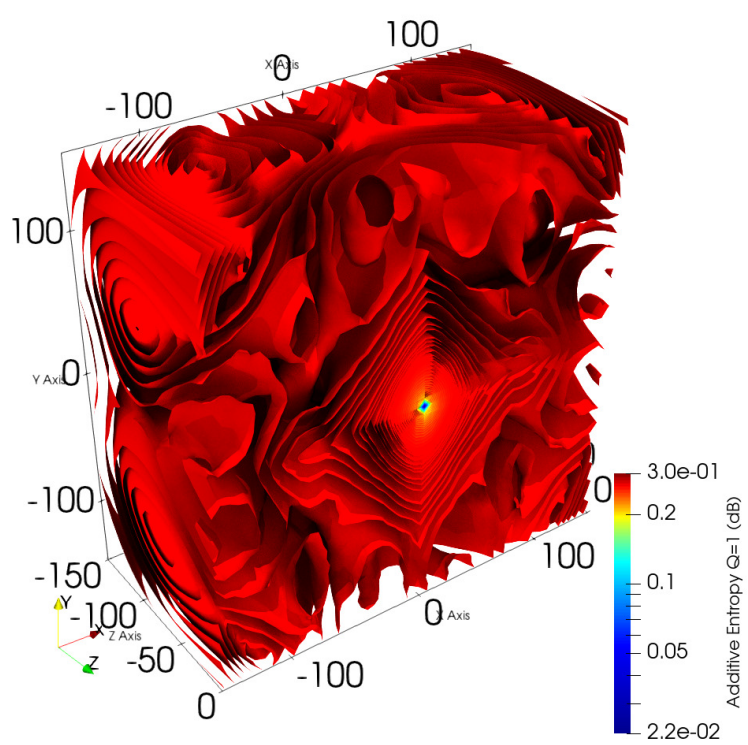

(c)

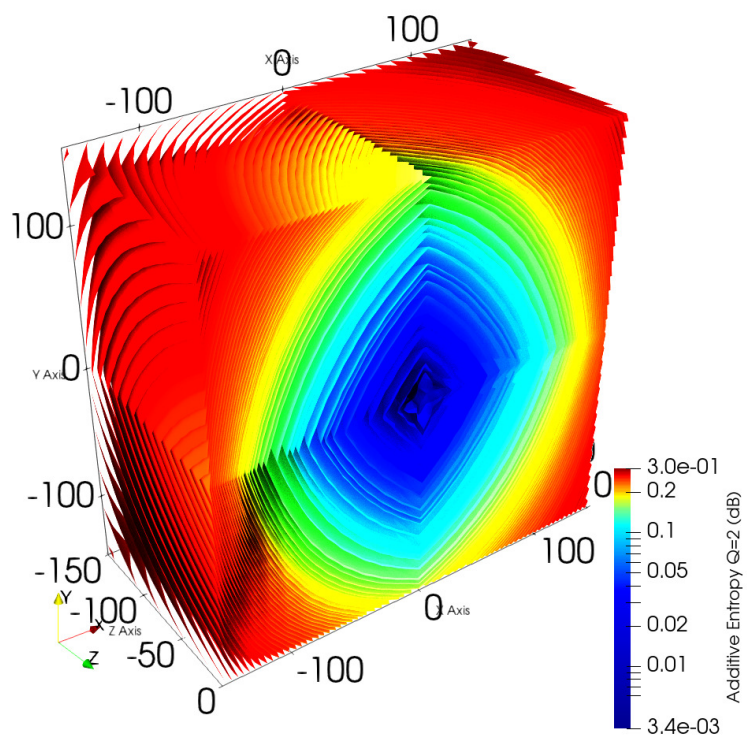

(b)

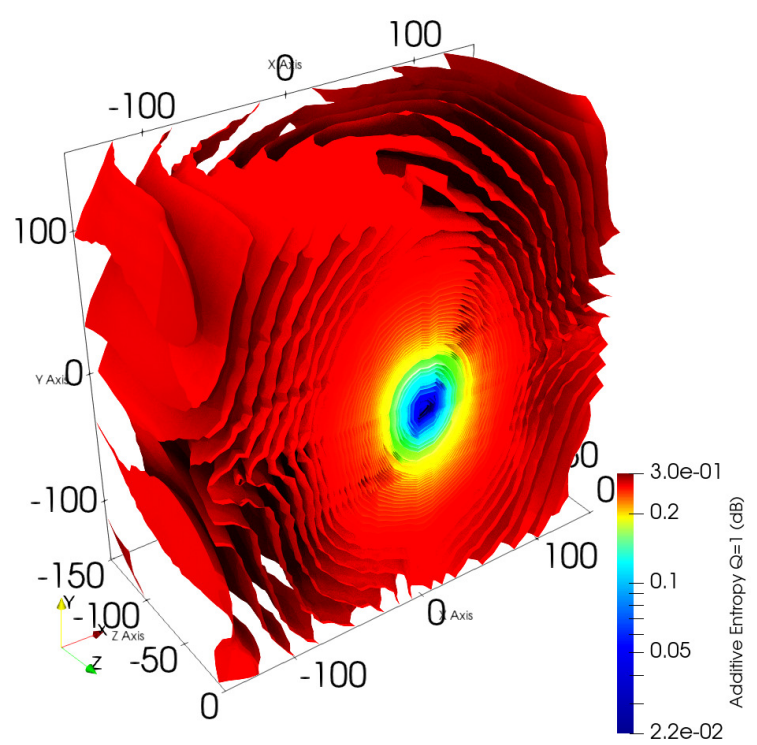

(d)

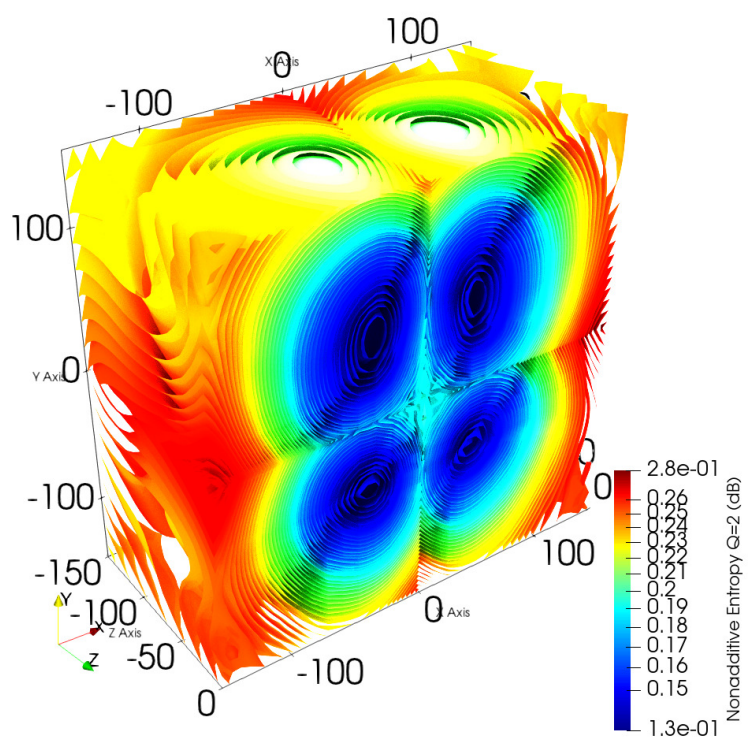

(a) Mattes (b) Shannon (c) Tsallis additive $(q=2)$ (d) Tsallis non-additive $(q=2)$. Source: author.

(d), that shows the MI isosurfaces for Tsallis metric when we apply eq. 1.36, with similar results to sec. 4.1 and sec. 4.2 , with four major extrema that narrow the capture range of this implementation to a tiny region. 
Figure $30-3 \mathrm{D}$ analysis Comparison between ITK Mattes and Shannon

(a)

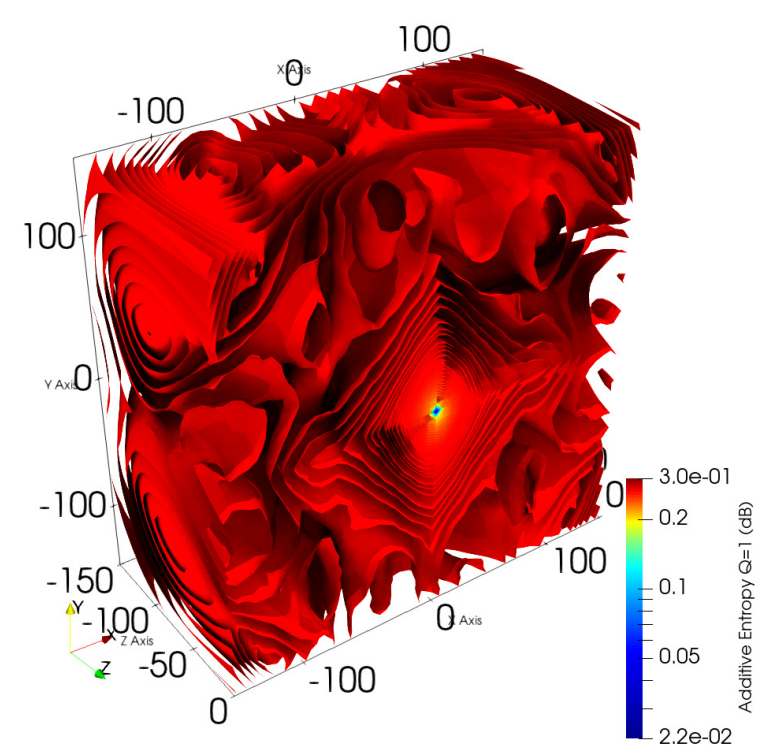

(c)

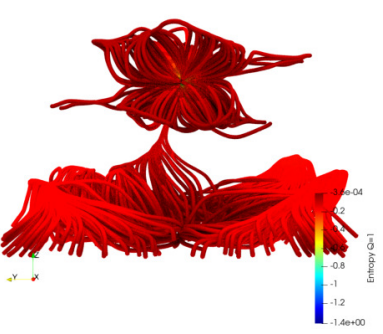

$(\mathrm{g})$

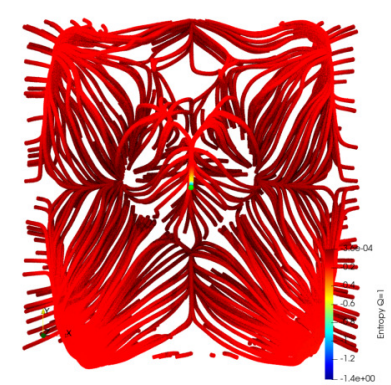

(d)

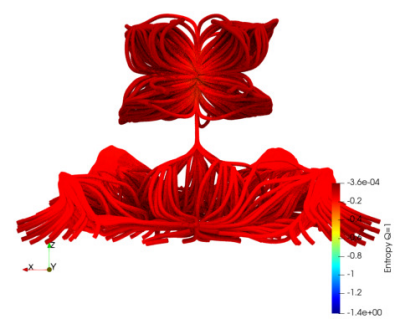

(h)

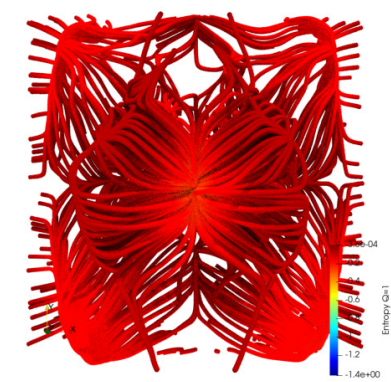

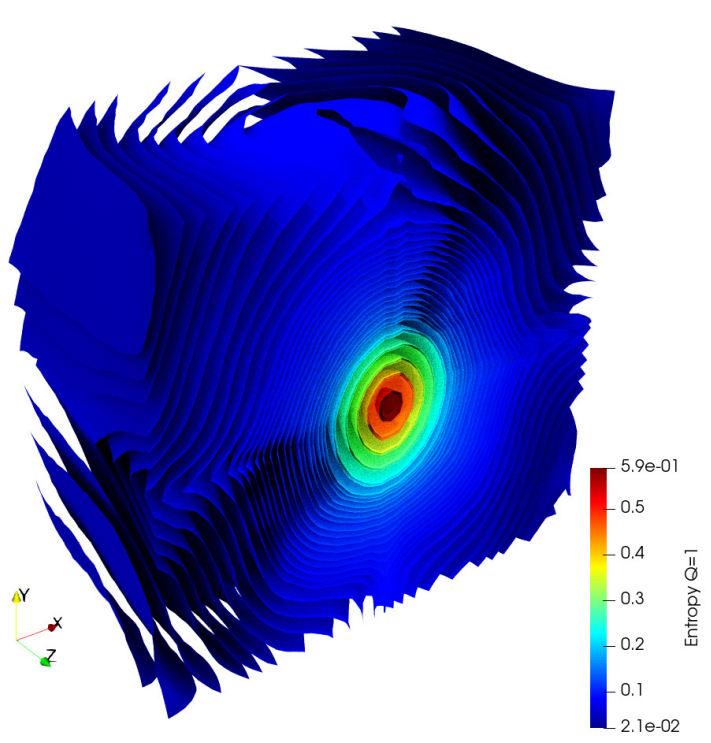

(e)

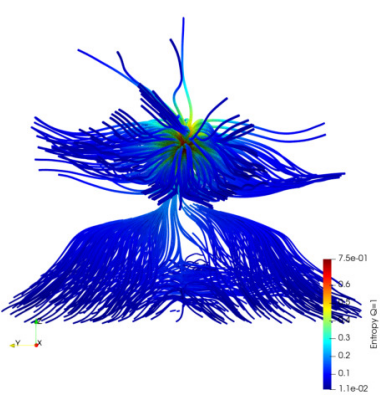

(i)

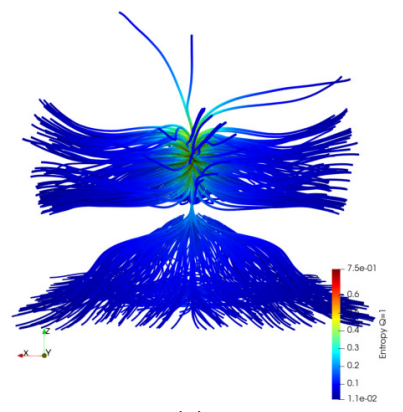

$(\mathrm{j})$

(b)
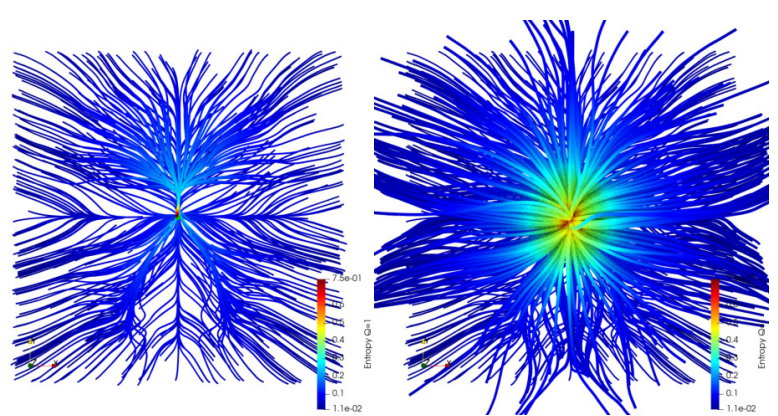

3D analysis Comparison between ITK Mattes and Shannon. Isosurfaces: (a) ITK Mattes (b) Shannon; Gradient Paths: horizontal views (c-d) Mattes (e-f) Shannon; Top views (d) Mattes (e) Mattes with center (f) Shannon (g) Shannon with center. Source: author. 
Figure $31-3 \mathrm{D}$ analysis Comparison between Shannon and Tsallis $(q=1.5)$

(a)

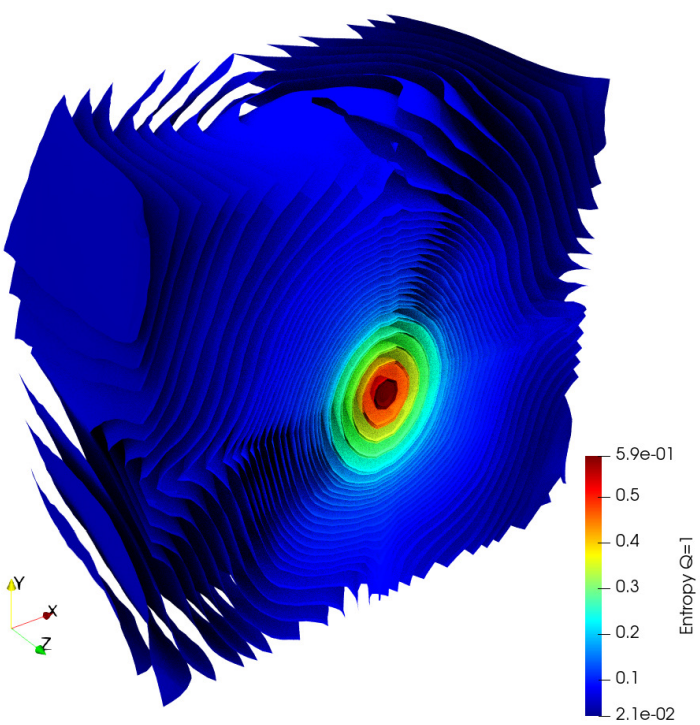

(c)

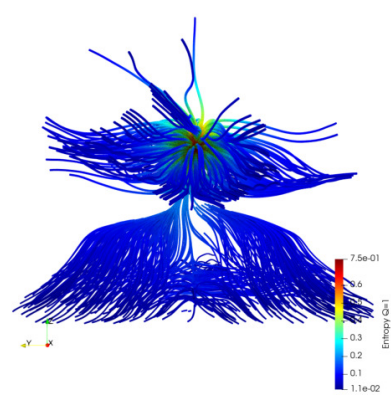

(g)

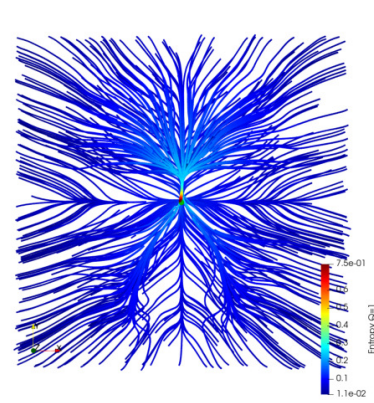

(d)

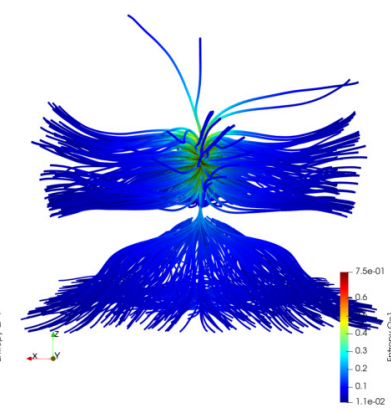

(h)

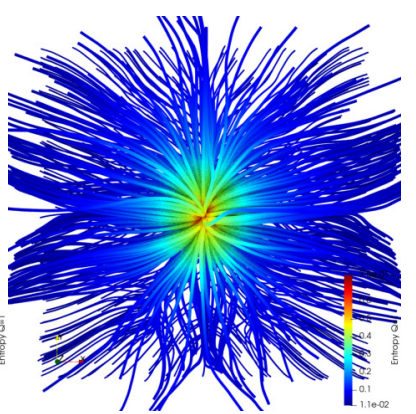

(b)

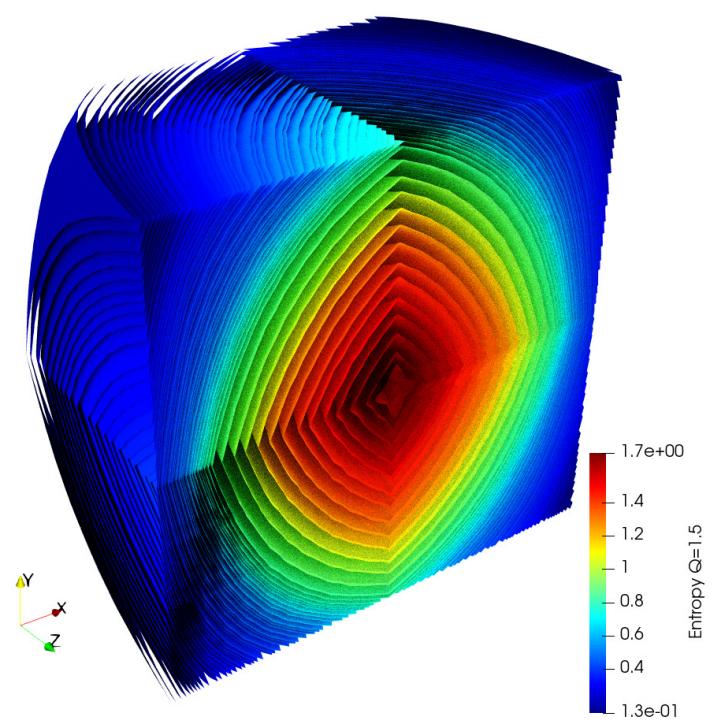

(e)

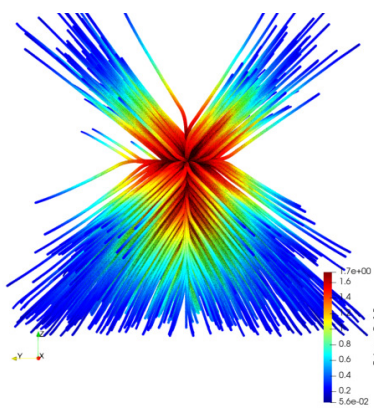

(i)

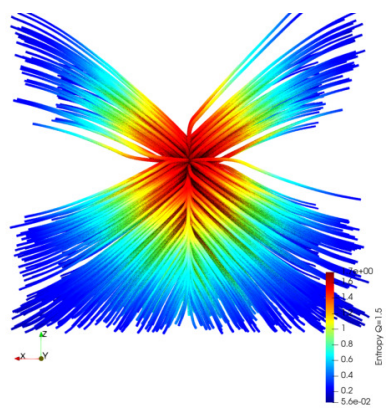

$(\mathbf{j})$
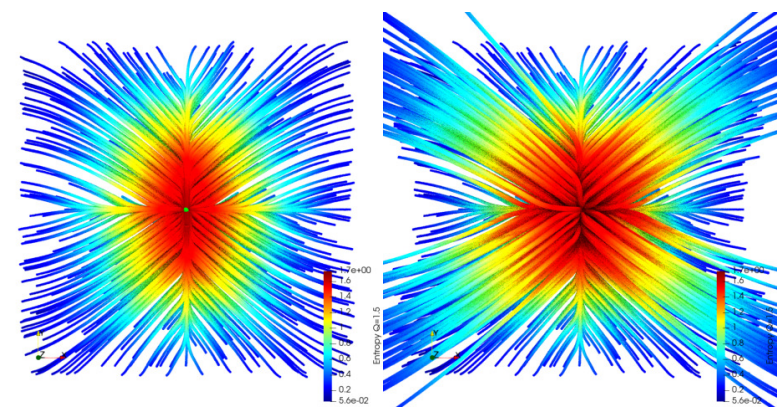

3D analysis Comparison between Shannon and Tsallis $(q=1.5)$. Isosurfaces: (a) Shannon (b) Tsallis; Gradient Paths: horizontal views (c-d) Shannon (e-f) Tsallis; Top views (d) Shannon (e) Shannon with center (f) Tsallis (g) Tsallis with center. Source: author. 


\subsubsection{Rotation}

\subsubsection{Versors}

Fig. 32(a-e) shows the isosurfaces for (a) ITK Mattes, (b) Shannon and (c-e) Tsallis metrics with the rotation transform using versors, in itkScaleSkewVersor3DTransform format. ITK Mattes shows a good capture range but non-spherical isosurfaces, whereas Shannon (b) presents some spherical symmetry and some extrema on the extremum of $x$, near the $y=0$ plane. Those Shannon's extrema seem to merge in (c) where the metric used is Tsallis with $q=2$, giving a narrower registration range and two strong extrema around the horizontal centerline, i.e., $y=z=0$ line, and smaller extrema on the vertical centerline, i.e., $x=z=0$ line. Tsallis with $q=0.5(\mathrm{~d})$ produces a narrower space, with a strong pole on the left horizontal centerline and a weak one on the right. The best result for Tsallis is using the entropic index of $q=1.05$, shown in (e), where all the horizontal extrema are absent, producing a wider capture range.

Fig. 32(f-h) are isosurfaces for Tsallis MI using histogram binning, allowing 6 bits of information. Shannon (f) shows some extrema on the diagonal corners of the space. In contrast, Tsallis additive with $q=2$ presents a broad capture range with some small extrema on the horizontal line, a result similar to (e) but more stable over the $q>1$ range. Using Tsallis non-additive with $q=2$, in (h), produces an almost perfect spherical symmetry and wider capture range in all studied cases.

\subsubsection{Degrees}

Fig. 33 shows results produced by using rotation in degrees instead of the versors used in the previous rotation results. ITK Mattes isosurfaces (a) show strong extrema on all corners that narrow the capture range of the metric. Furthermore, the surfaces' symmetry is present only for small angles $\left(\theta<45^{\circ}\right)$, showing more turbulence as the angles grow. Shannon (b) also has a similar result with the corner extrema and the turbulence as the angle grows. For the gradient paths in images $(\mathrm{c}-\mathrm{j})$, when visualized by the sides from axis $x$ or $y$, we can see for both ITK Mattes (c-d), and Shannon (e-f) cases the center block disconnected from the bottom paths. This disconnection represents that registrations starting on the bottom space will not register to our center. The main difference between ITK Mattes and Shannon is a more connected center block, showing a wider capture range for Shannon (e-f) than the narrower center block in ITK Mattes (c-d). The visualizations from the $z$ axis (g-j) are very similar, corroborating the affirmations for images (c-f).

Fig. 34 shows, in a similar way to fig. 33, a comparison between Shannon and Tsallis additive with $q=1.3$. The Tsallis isosurfaces (b) are farther from spherical shapes than the Shannon ones (a), with no significant visual difference in the spaces. For the gradient paths $(c-j)$, we can see a small improvement on the capture range, represented 
Figure $32-3 \mathrm{D}$ isosurfaces using rotation with versors/quaternions
(a) ITK Mattes
(b) Shannon
(c) Tsallis $\mathrm{Q}=2$
(d) Tsallis $\mathrm{Q}=0.5$
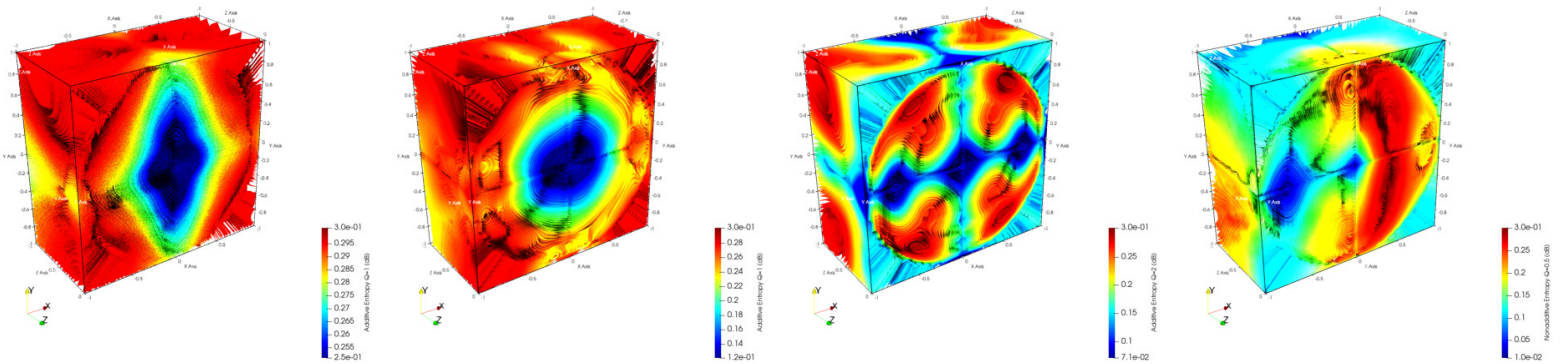

(e) Tsallis $\mathrm{Q}=1.05$

(f) Shannon 6 bits

(g) Tsallis Additive $\mathrm{Q}=26(\mathbf{h})$ Tsallis Nonadditive bits $\quad \mathrm{Q}=26$ bits
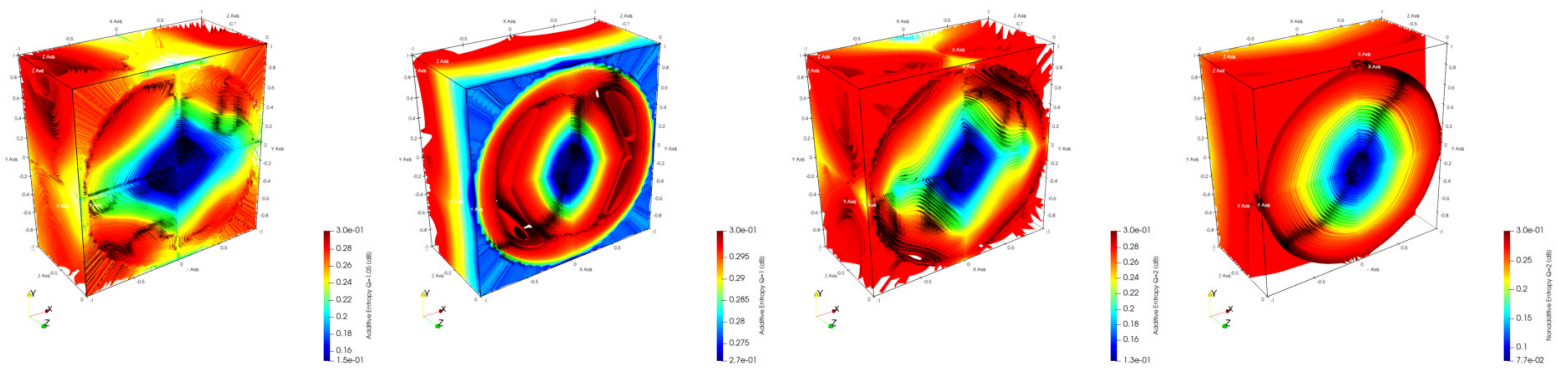

Source: author.

by the size of the central block, for the Tsallis metrics (e-f, i-j). However, visually the difference is negligible. 
Figure 33 - 3D analysis Comparison between ITK Mattes and Shannon for rotation with degrees.

(a)

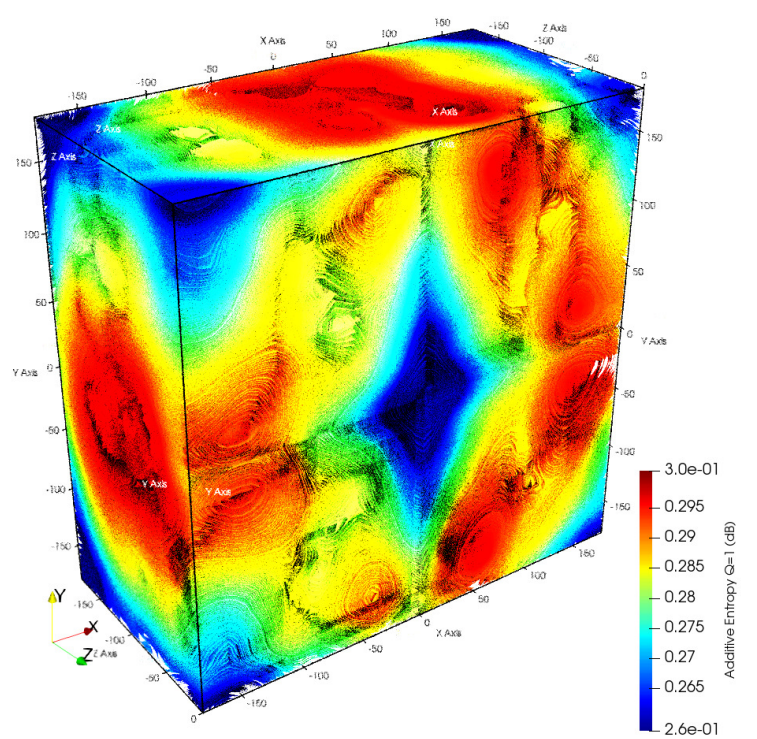

(c)

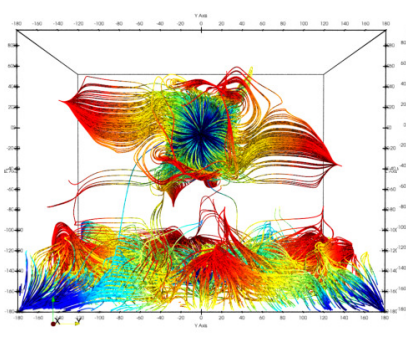

(g)

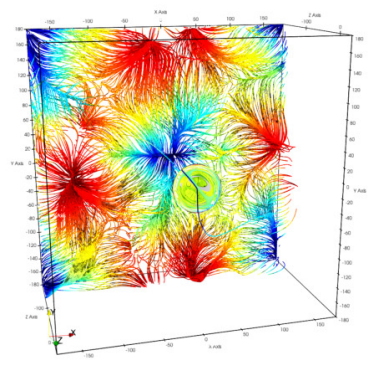

(d)

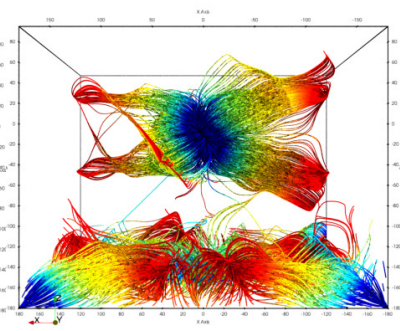

(h)

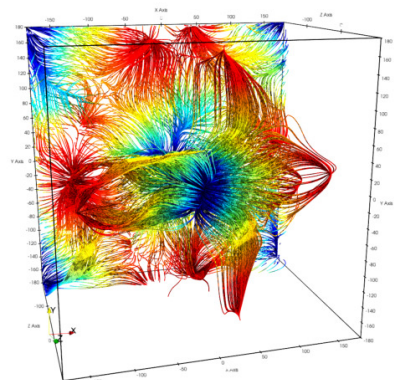

(b)

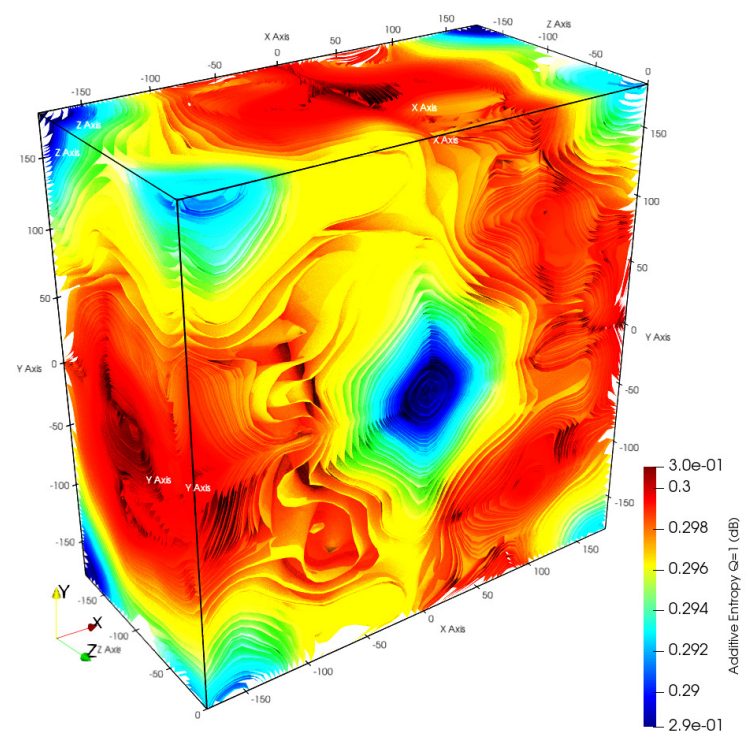

(e)

(f)

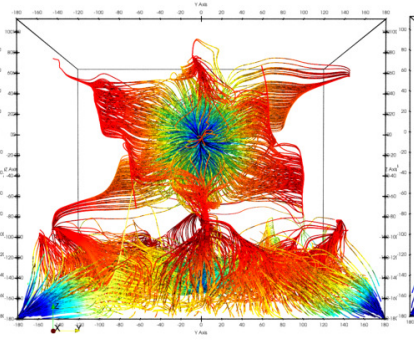

(i)

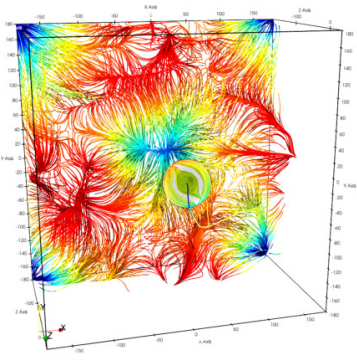

(j)

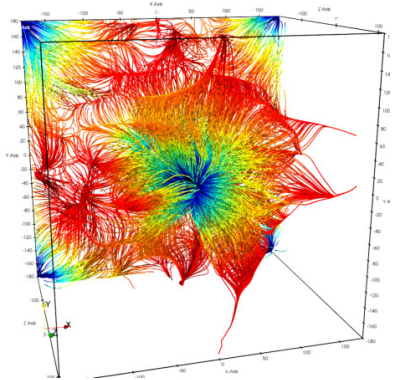

3D analysis Comparison between ITK Mattes and Shannon for rotation with degrees. Isosurfaces: (a) ITK Mattes (b) Shannon; Gradient Paths: horizontal views (c-d) Mattes (e-f) Shannon; Top views (d) Mattes (e) Mattes with center (f) Shannon (g) Shannon with center. Source: author. 
Figure $34-3 \mathrm{D}$ analysis Comparison between Shannon and Tsallis ( $q=1.3,4$ bits binning).

(a)

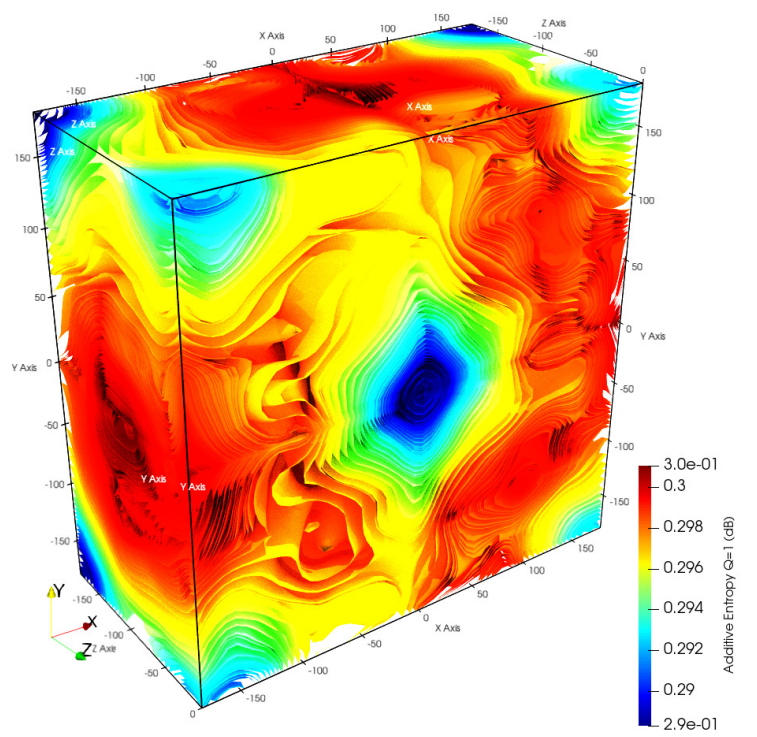

(c)

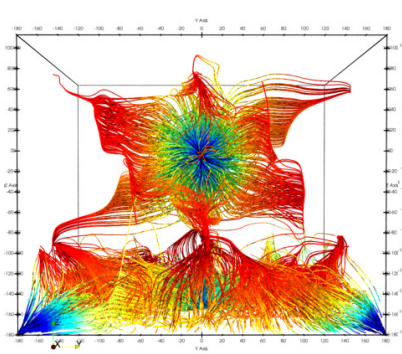

(g)

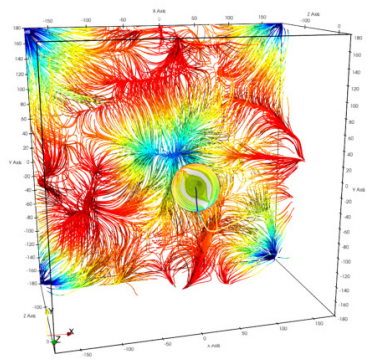

(d)

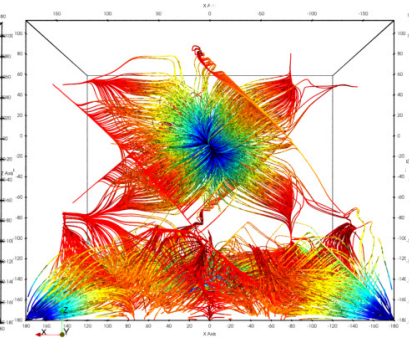

(h)

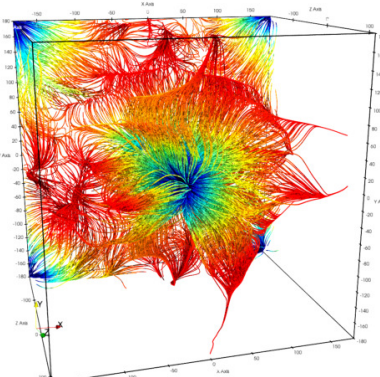

(b)

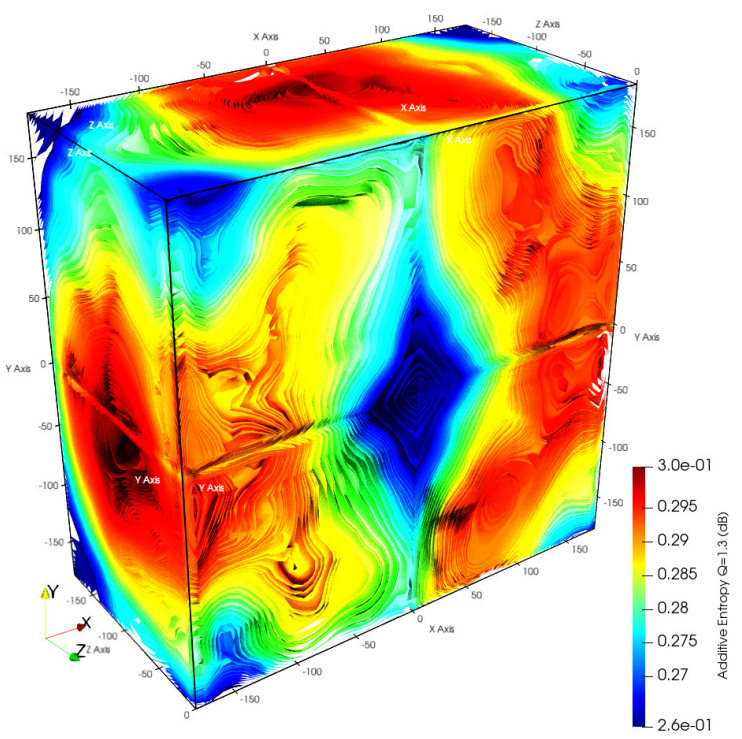

(e)

(f)

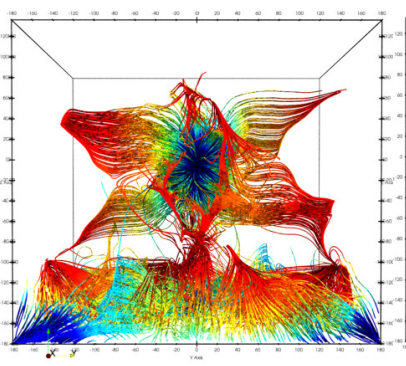

(i)

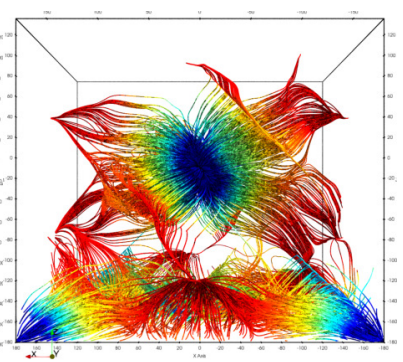

$(\mathbf{j})$
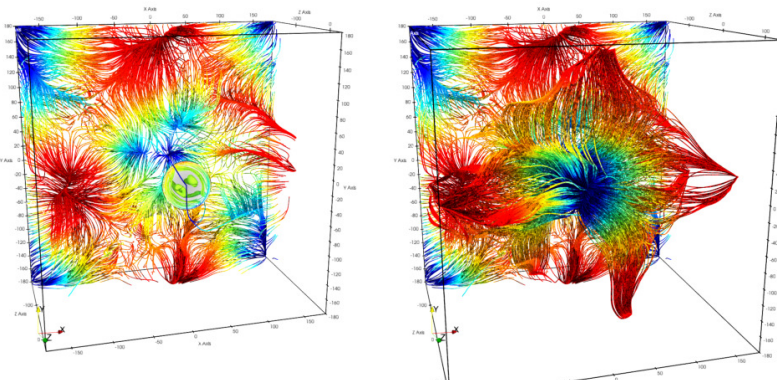

3D analysis Comparison between Shannon and Tsallis ( $q=1.3,4$ bits binning). Isosurfaces: (a) Shannon (b) Tsallis; Gradient Paths: horizontal views (c-d) Shannon (e-f) Tsallis; Top views (d) Shannon (e) Shannon with center (f) Tsallis (g) Tsallis with center. Source: author. 
Fig. 35 shows how the metric output space changes regarding changes in the entropic index $q$. The top row (a-e) are Tsallis non-additive metrics, and the bottom row (f-j) are Tsallis additive metrics, with both presenting a similar behavior. In the non-additive Tsallis row, lowering the entropic index, i.e., $q<1$, initially gives a better space and wider capture range, as $q=0.95$ shown in (b). However, as we go further, as $q=0.7$ in (c), we start to see some periodic behavior and some extrema emerging on $90^{\circ}$ and $-90^{\circ}$ regions, and a much narrower capture range than (b). Going the $q>1$ direction narrows the capture range, as in $q=1.05$ in $(\mathrm{d})$, furthermore, we have new extrema emerging, but now on $45^{\circ}$ and $-45^{\circ}$ regions, almost inverse than the result shown in (a).

The bottom row of fig. 35 are Tsallis additive metrics, with similar problems as non-additive, the extrema at $90^{\circ}$ now appear on $q>1$ and the $45^{\circ}$ extrema on $q<1$, inverting the situation from the top row.

Fig. 36 visualizes the effects of histogram binning on the metrics functions. On the top row is presented the Shannon metric with binning varying from 12 bits to 2 bits, noting that 12 bits is the resolution of the HCP images and means no histogram binning is made in practice. Going from 12 bits (a) to 6 bits (c), the turbulence on the isosurfaces diminishes. Going lower than 6 bits the turbulence grows again, as in 4 bits (d) and 2 bits (e), noting that even with only 2 bits of information, we can still register a large portion of the space.

On the top row of fig. 36 are presented the changes on the metric output space, using 4 bits histogram binning and changing the $q$ entropic index of the Tsallis additive metric. The most notable difference is the absence of periodicity, except the natural one as rotations of $180^{\circ}$ and $-180^{\circ}$ are the same. Using histogram binning also seems to diminish the effect of the entropic index on the metric output space. Making it more robust to changes, as we can see, images (g-j) are very similar. Only image (f), using $q=0.7$, have a different space structure. 
Figure 35 - 3D isosurfaces using degrees rotation with no histogram binning.

(a) Tsallis Nonaddi(b) Tsallis Nonaddi(c) Tsallis Nonaddi(d) Tsallis Nonaddi(e) Tsallis Nonadditive $\mathrm{Q}=0.7$ tive $\mathrm{Q}=0.95$ tive $\mathrm{Q}=1$ tive $\mathrm{Q}=1.05$ tive $\mathrm{Q}=1.5$

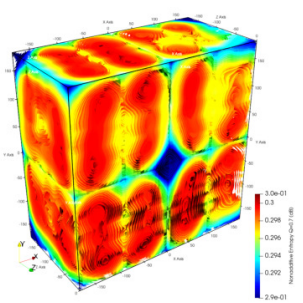

(f) Tsallis Additive(g) Tsallis Additive( $\mathrm{Q}=0.7$

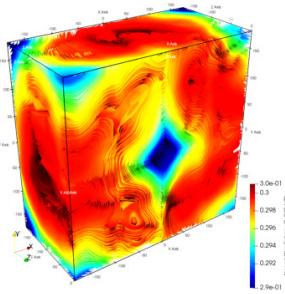

$\mathrm{Q}=0.95$

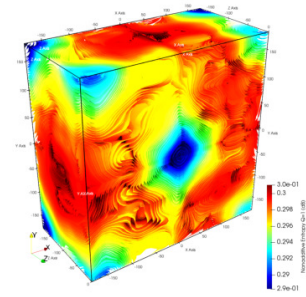

(h)

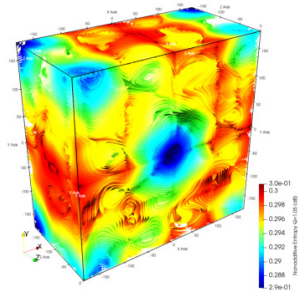

(i) Tsallis Additive $(\mathrm{j})$

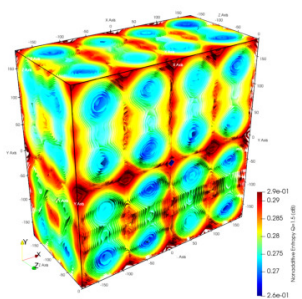

(j) Tsallis Additive $\mathrm{Q}=1.05$

$\mathrm{Q}=1.5$
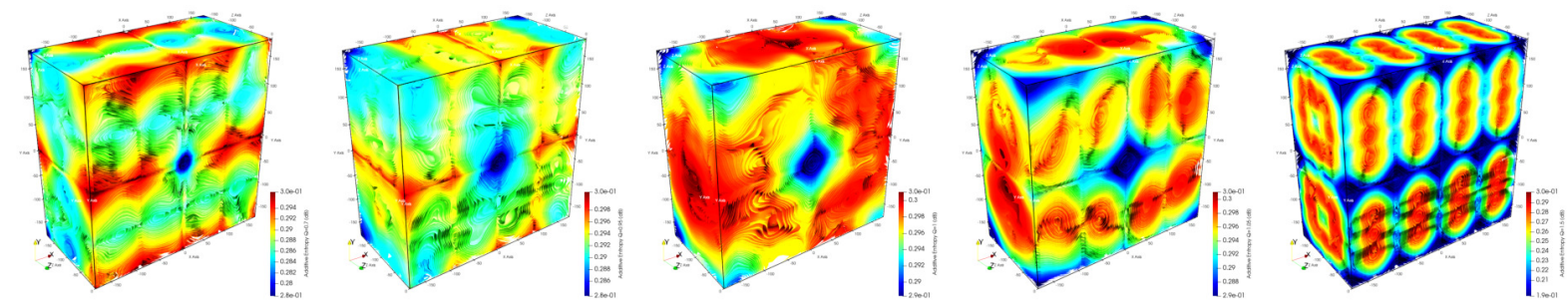

Source: author.

Figure $36-3 \mathrm{D}$ isosurfaces using degrees rotation with histogram binning.
(a) Shannon 12 bit
(b) Shannon 8 bits
(c) Shannon 6 bits
(d) Shannon 4 bits
(e) Shannon 2 bits

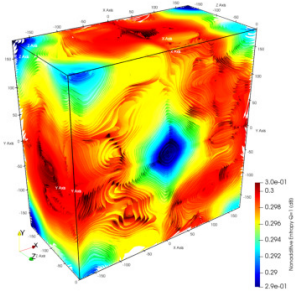

(f) Tsallis Additive (g) Tsallis Adtitio $\mathrm{Q}=0.74$ bits

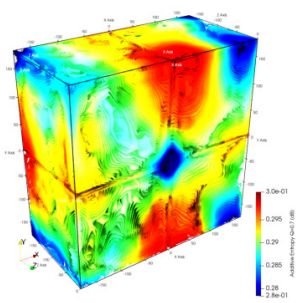

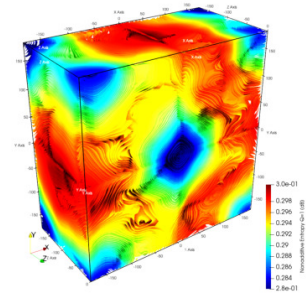

$\mathrm{Q}=0.954$ bits

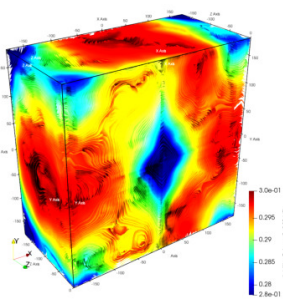

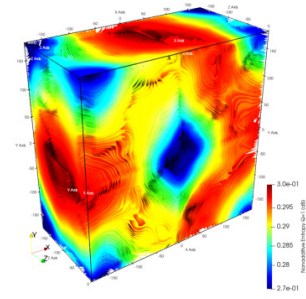

(h) Tsallis Additive $(i)$

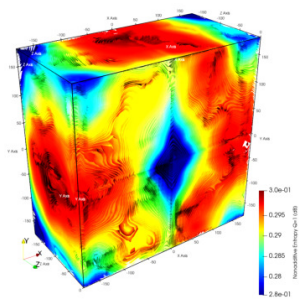

(i) Tsallis Additive $(\mathbf{j})$

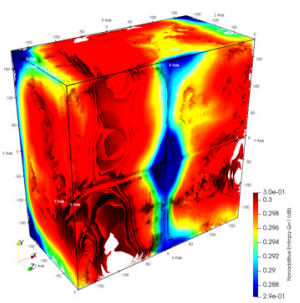

$\mathrm{Q}=1.054 \mathrm{bits}$

$\mathrm{Q}=1.34$ bits

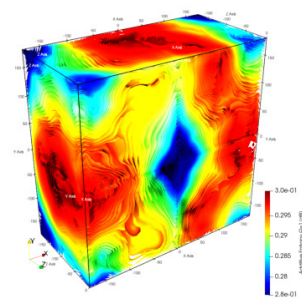

Source: author.
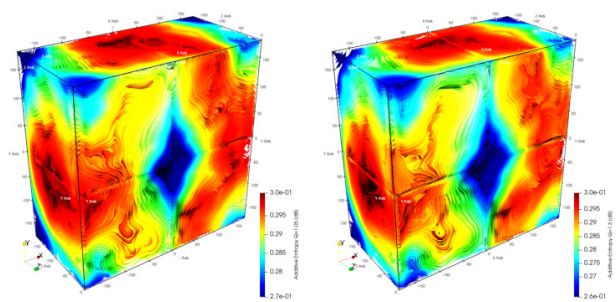


\subsubsection{Scaling}

Fig. 37 shows the metrics response to scaling transforms, with ITK Mattes (a) and Shannon (b) outperforming Tsallis by a large margin. ITK Mattes has smooth surfaces with good symmetry, while Shannon has less symmetry but still has a good and wide capture range for registration. Tsallis in all versions tested, additive and non-additive, and with $q<1$ or $q>1$, performed poorly in the scaling experiment, with narrow capture range and very interesting properties as the isosurfaces became almost horizontal or vertical in some quadrants (images c-d). Using histogram binning provides better performance, as seen in image (e) with 10 bits and $q=0.9$ in the Tsallis additive metric. Another property worth noting in the experiments is that additive (d) and non-additive (c) are almost inverted versions of each other.

\subsubsection{Skewness}

Fig. 38 shows the metrics response to skewness transforms, with all metrics performing well, only with slight differences on the isosurfaces smoothness, with ITK Mattes (a) being more turbulent while Shannon (b) and Tsallis non-additive (c) having isosurfaces with a spherical-like shape. The Tsallis additive metric (d) presents a slightly turbulent space, yet capable of capturing a wide registration range, like all other metrics. 
Figure $37-3 \mathrm{D}$ isosurfaces using scaling transformations.

(a) ITK Mattes

(b) Shannon

(c) Tsallis Nonaddi(d) Tsallis Additive(e) Tsallis Additive
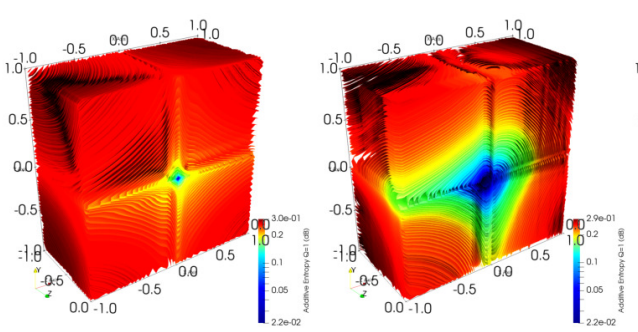

tive $\mathrm{Q}=2$ $\mathrm{Q}=2$ $\mathrm{Q}=0.9$ 10bits
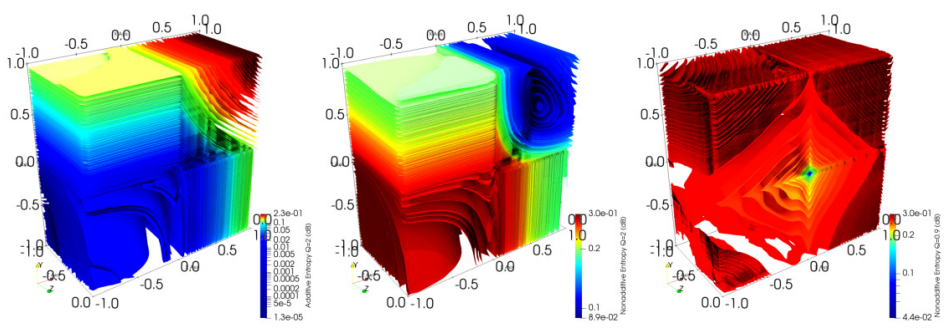

Source: author.

Figure $38-3$ D isosurfaces using skewness transformations.
(a) ITK Mattes
(b) Shannon
(c) Tsallis Nonadditive
$\mathrm{Q}=2$
(d) Tsallis Additive $\mathrm{Q}=2$
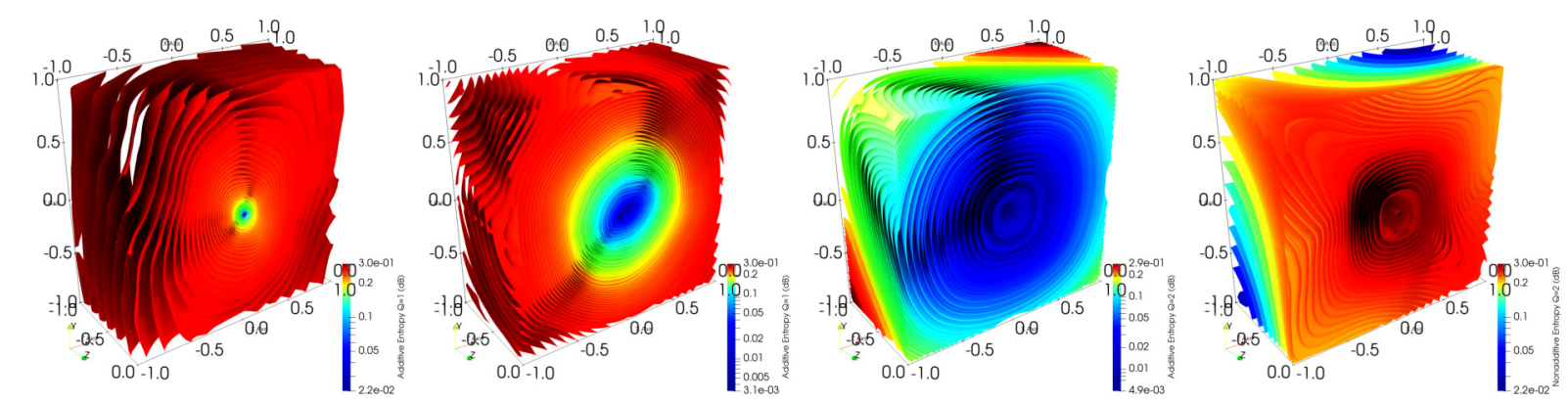

Source: author. 


\subsection{Registration capacity}

Fig. 39 shows the line plots for the registration capacity of the Tsallis metrics, evaluated as described in sec. 3.3.3. The dashed horizontal blue line is the Mattes' registration capacity, plotted to serve as a baseline. The left graphs are with all the metrics analyzed, and the right graphs are limited only to metrics that outperform the Mattes metric.

For the translation transformations, shown in fig. 39(a-b), Tsallis outperform Mattes on all levels of histogram binning, and in general, the additive versions (solid lines) outperform their non-additive counterparts (dashed lines). Except for the 1-bit histogram binning, we have very stable metrics in the Shannon region $(q \rightarrow 1)$. Also, the metrics performed better as more information was passed to them by the histogram binning, with a higher bit binning outperforming lower ones. The details in fig. 39b show, clearly, that the $q>1$ region performs better than $q<1$ for those metrics.

For the rotation transformations shown in fig. 40(a-b), the most challenging problem tested, we have different behavior from translation, as metrics having a high number of bins performed worse than metrics with fewer bins. The only metrics that outperform Mattes are using histogram binning with $1-4$ bits, with the best overall being the 4 bits version with non-additive Tsallis and $q=1.3$ (fig. 40b).

For the scaling transformations, shown in fig. 41(a-b), the Mattes level is high at 99\% capacity. However, almost all metrics tested can outperform it with good $q$ settings, with only the 1-bit binning performing poorly. The 2 bits have a poor Shannon capacity but improve as we go in the $q>1$ region. The difficulty of this transformation is that as we go further on the $q>1$ region, the metric's capacity start to decay, so we have a good capacity region around the $1.01 \leq q \leq 1.09$ region, with the best $q$ depending on the histogram binning used.

For the skewness transformations, shown in fig. 42(a-b), the Mattes level is the highest, with $100 \%$ capacity, and visually all metrics reach this level in the Shannon range, becoming stable in the $1 \leq q \leq 1.1$ range. Since Shannon and Mattes have a total capacity, it is impossible to outperform it, only to tie their capacity.

The same data used on these line plot figures are also used to generate fig. 43, table 2, and table 3. Some results for Rényi entropy are also included and mirrors Tsallis entropy in some ways. However, in general, we can have a good registration capacity using Tsallis alone.

Fig. 43 visualized the data in a heatmap manner, in a full colorbar scaling in (a) and a range-limited colorbar in (b), to better visualize the best $q$ values and the small changes around the best capacity level. In addition, the rotation metrics are detached in fig. $43 \mathrm{~b}$ since they do not reach a full, $100 \%$ registration capacity and needed a different colorbar scaling to visualize their best levels. 
Table 2 show the raw data from the naive algorithm for the $q \leq 1$ region, whereas table 3 shows for the $q \geq 1$ region. 
Figure 39 - Tsallis' translation registration capacity as functions of the entropic index $q$

(a) Translation

\section{Metrics registration percentage as function of $q$ entropic index}

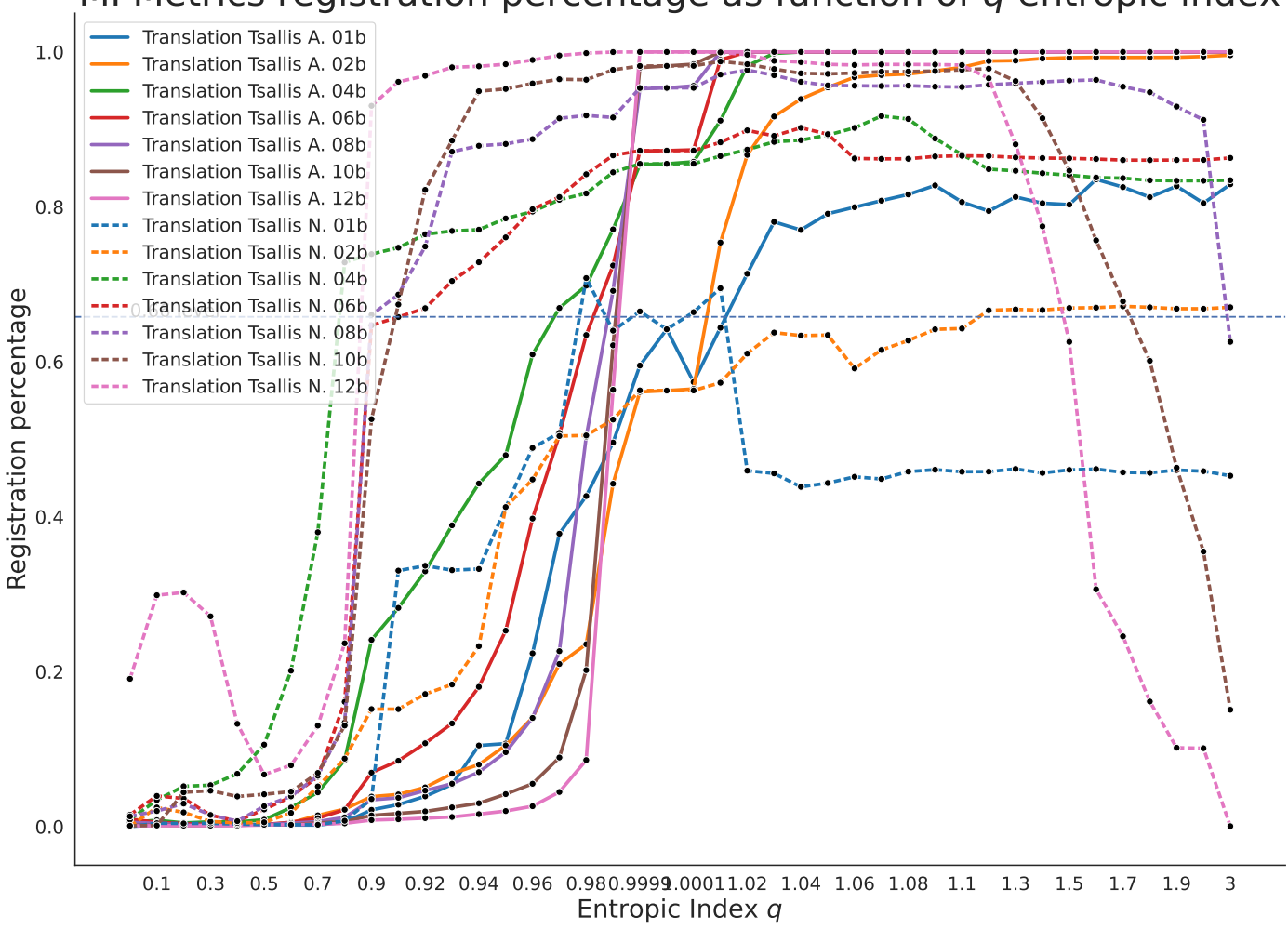

(b) Translation limited

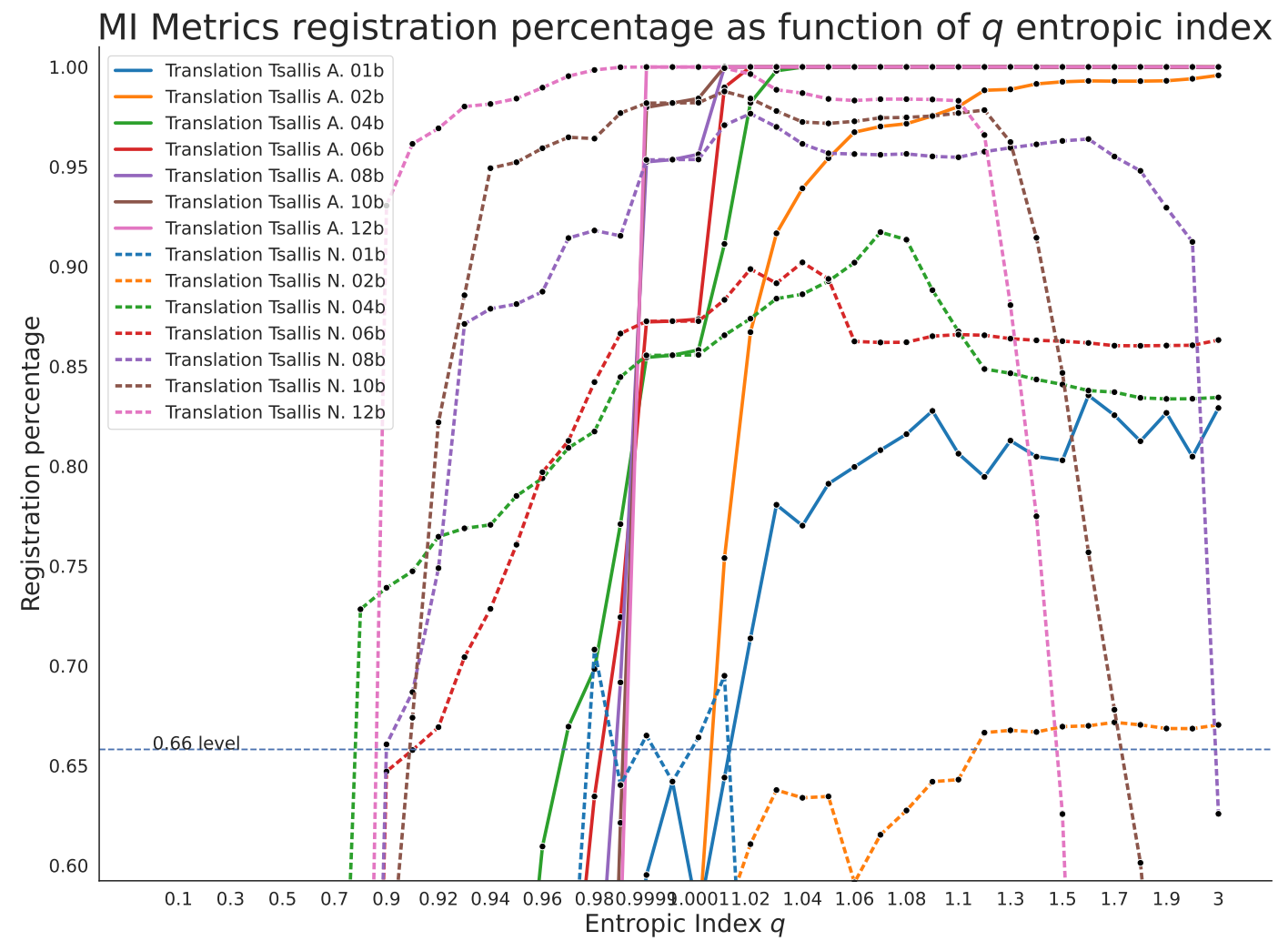

Tsallis' metrics registration capacity as functions of the entropic index $q$ : (left) full plot (right) limited to the metrics that outperform ITK Mattes metric for that transformation. Source: author. 
Figure 40 - Tsallis' rotation registration capacity as functions of the entropic index $q$

(a) Rotation

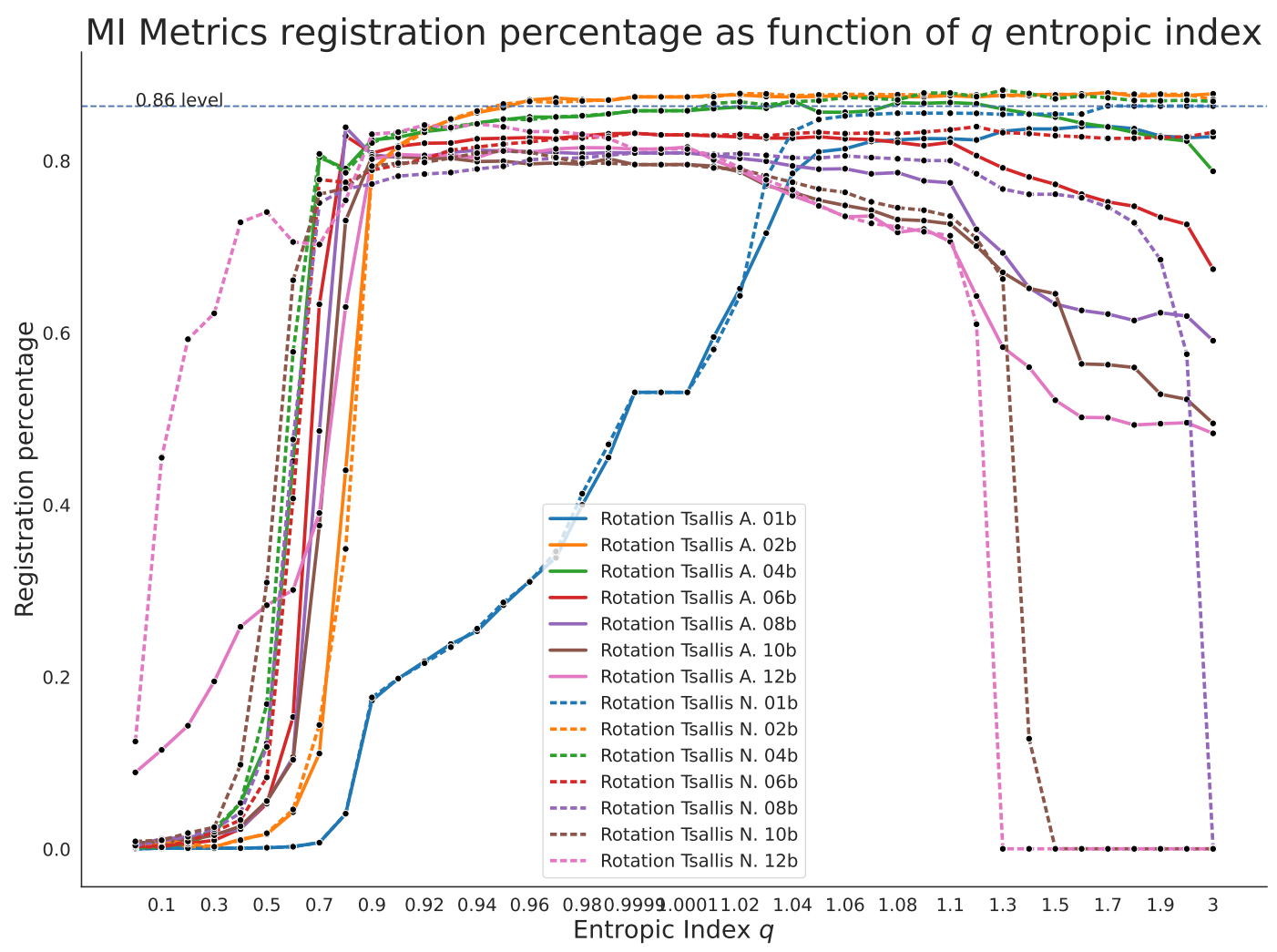

(b) Rotation limited

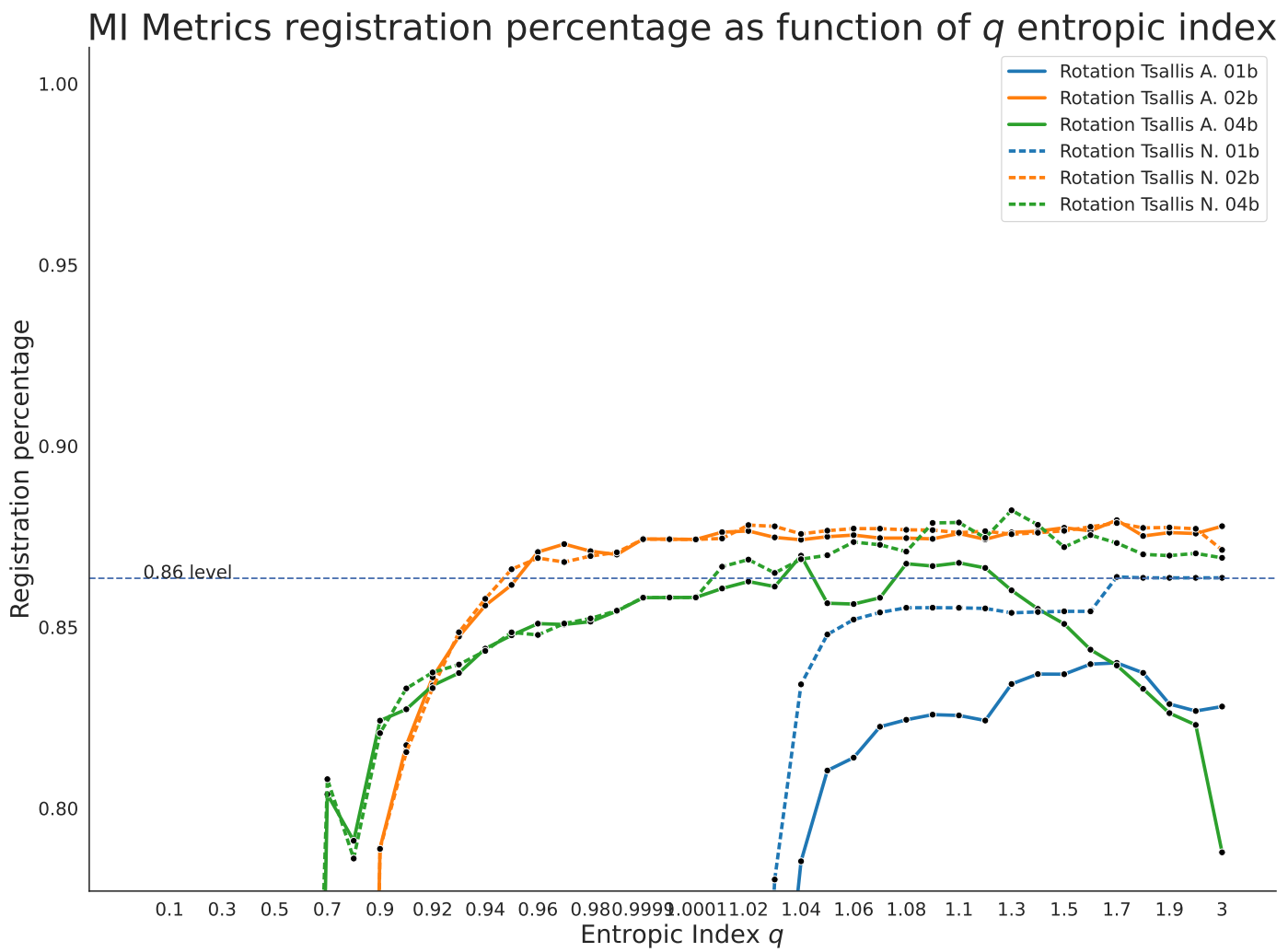

Tsallis' metrics registration capacity as functions of the entropic index $q$ : (left) full plot (right) limited to the metrics that outperform ITK Mattes metric for that transformation. Source: author. 
Figure 41 - Tsallis' scaling registration capacity as functions of the entropic index $q$

(a) Scaling

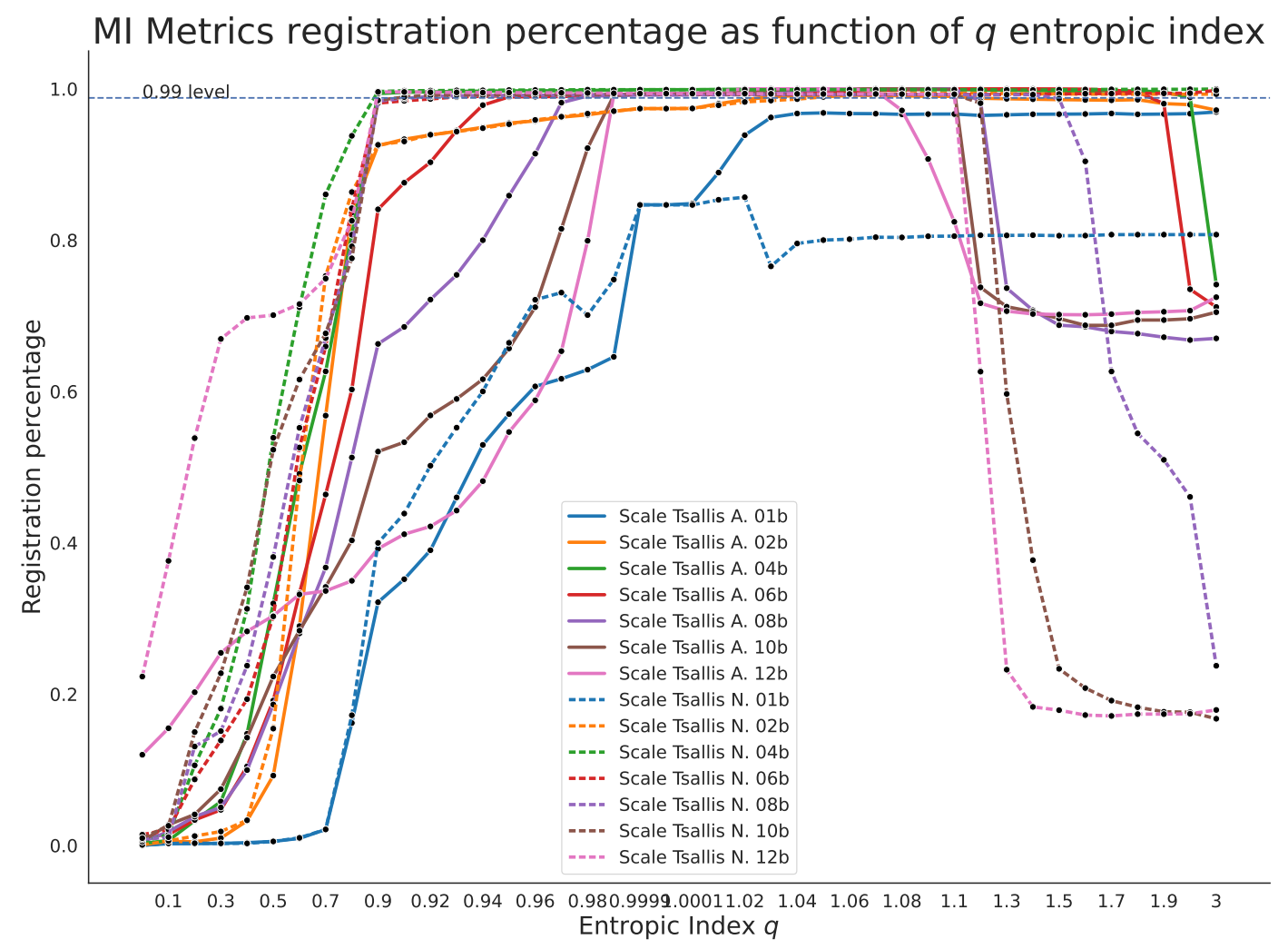

(b) Scaling limited

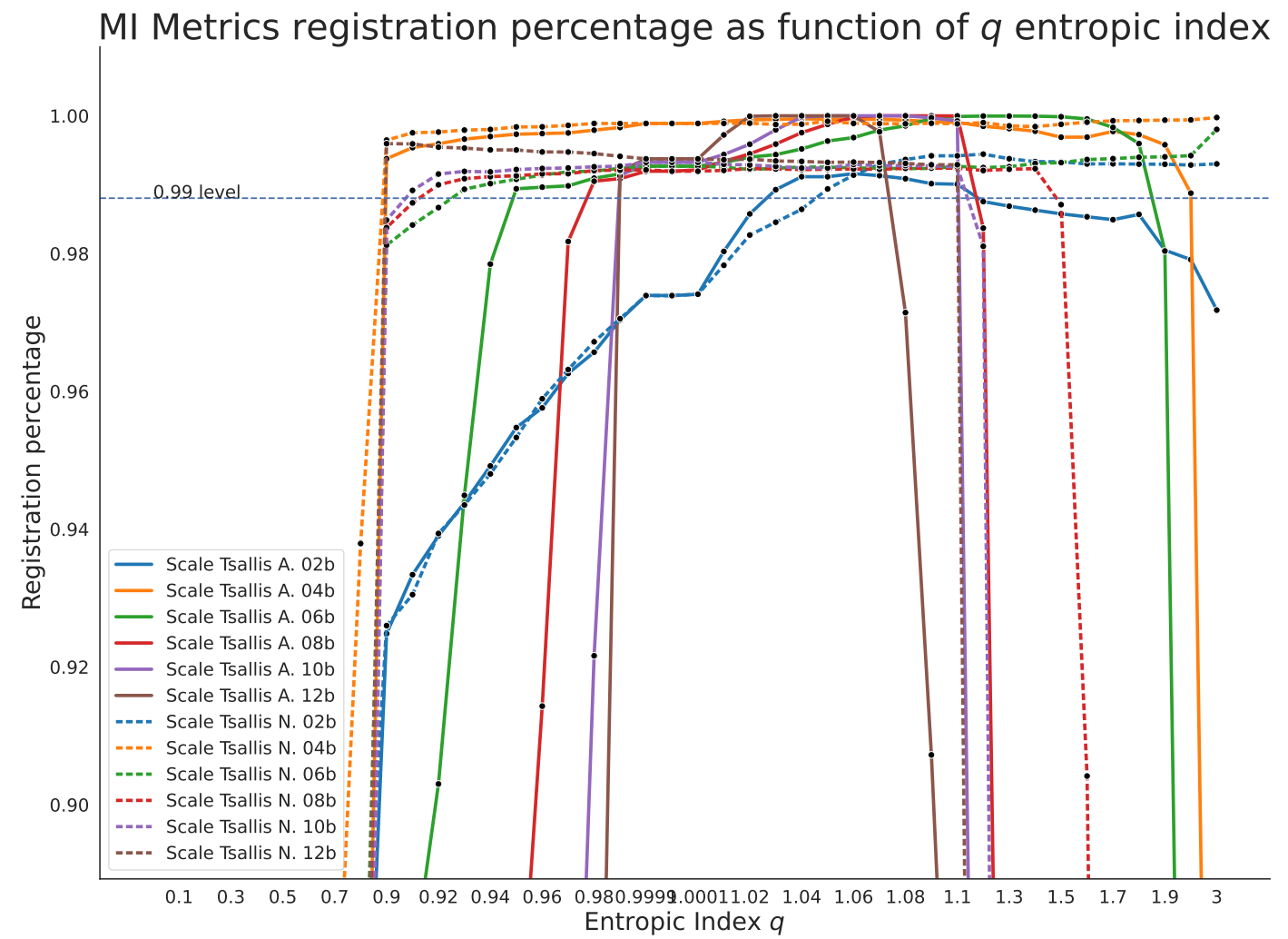

Tsallis' metrics registration capacity as functions of the entropic index $q$ : (left) full plot (right) limited to the metrics that outperform ITK Mattes metric for that transformation. Source: author. 
Figure 42 - Tsallis' skewness registration capacity as functions of the entropic index $q$

(a) Skewness

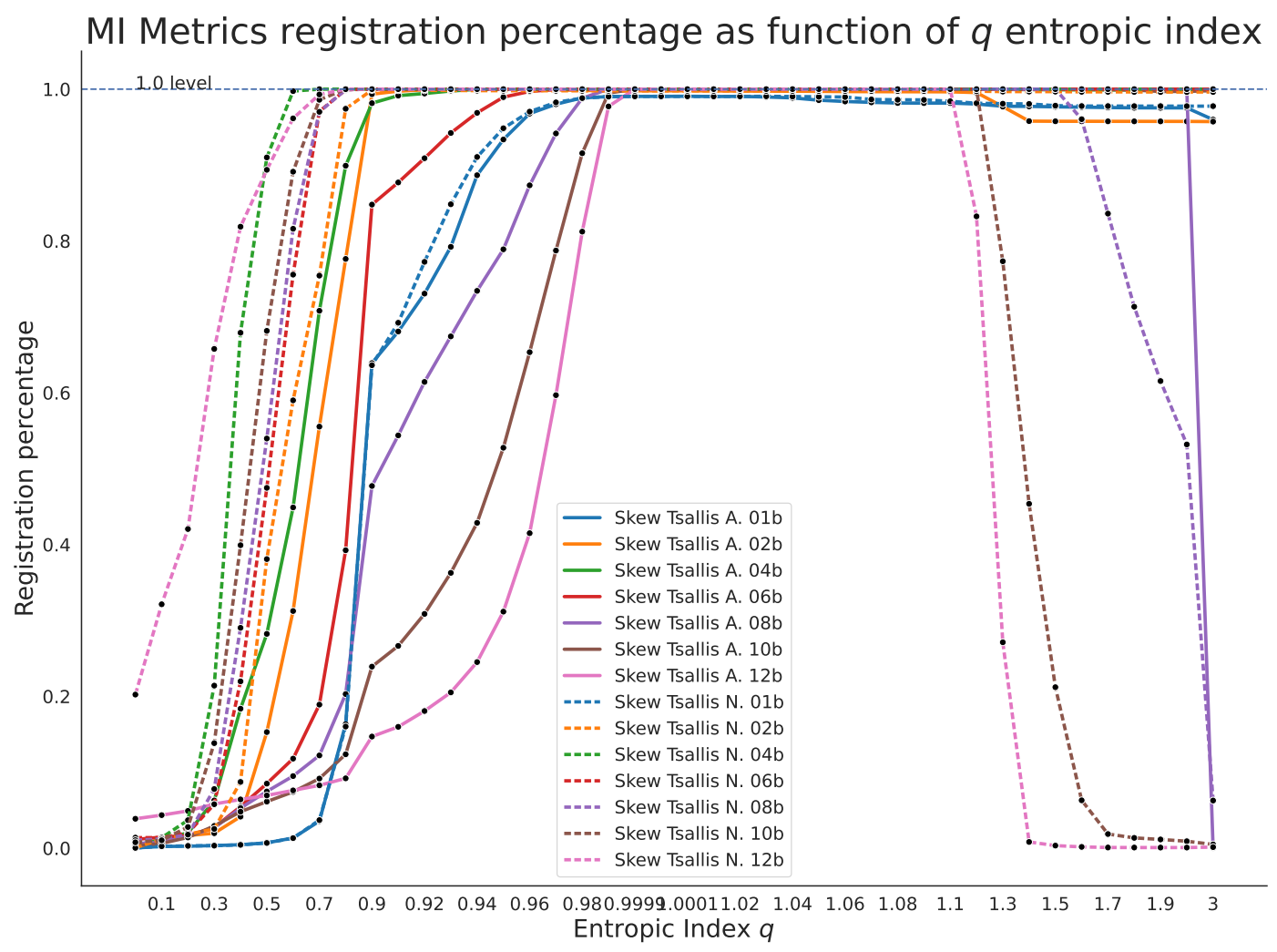

(b) Skewness limited

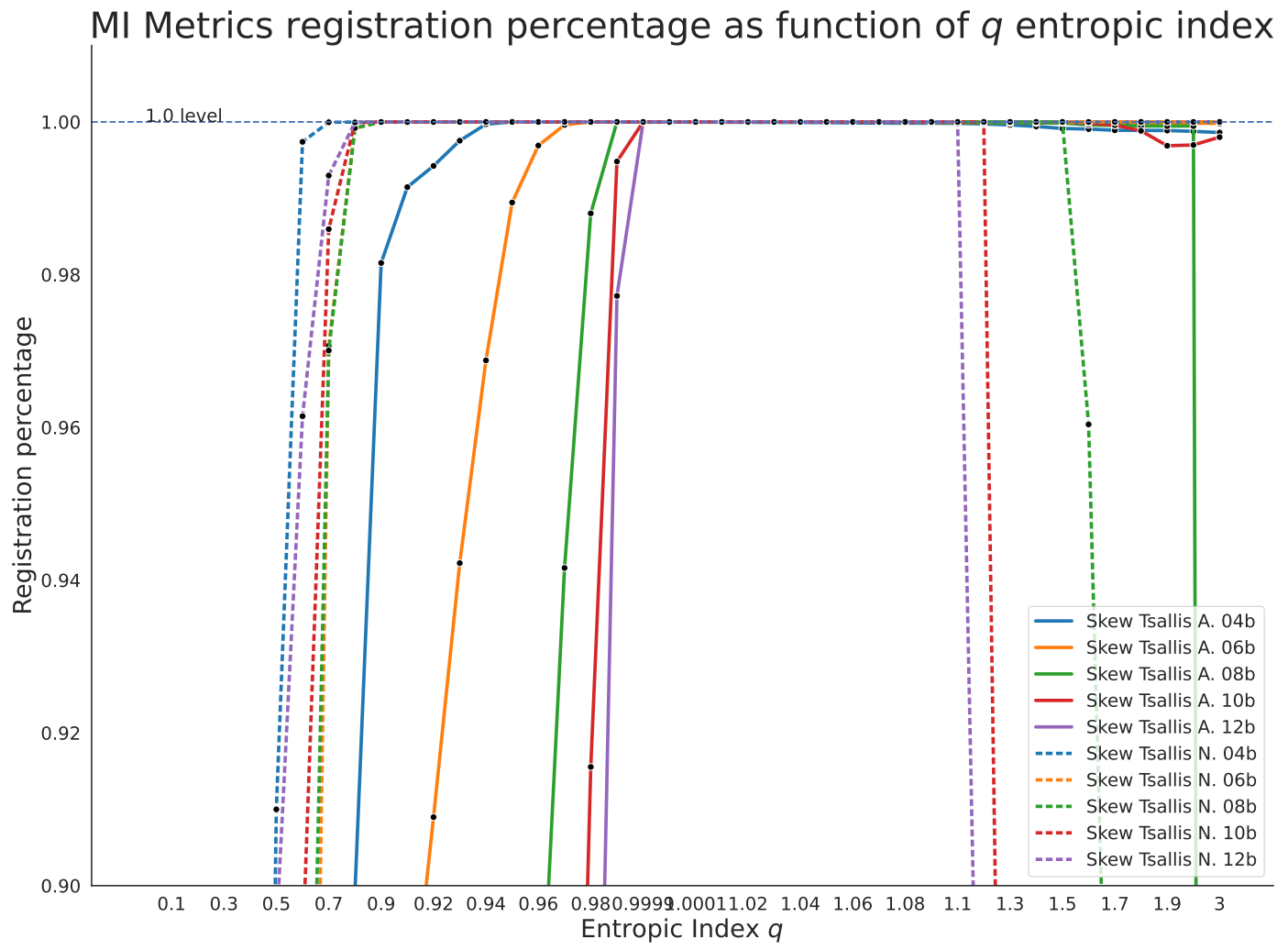

Tsallis' metrics registration capacity as functions of the entropic index $q$ : (left) full plot (right) limited to the metrics that outperform ITK Mattes metric for that transformation. Source: author. 
Figure 43 - Metrics heatmaps

(a) Full colorbar

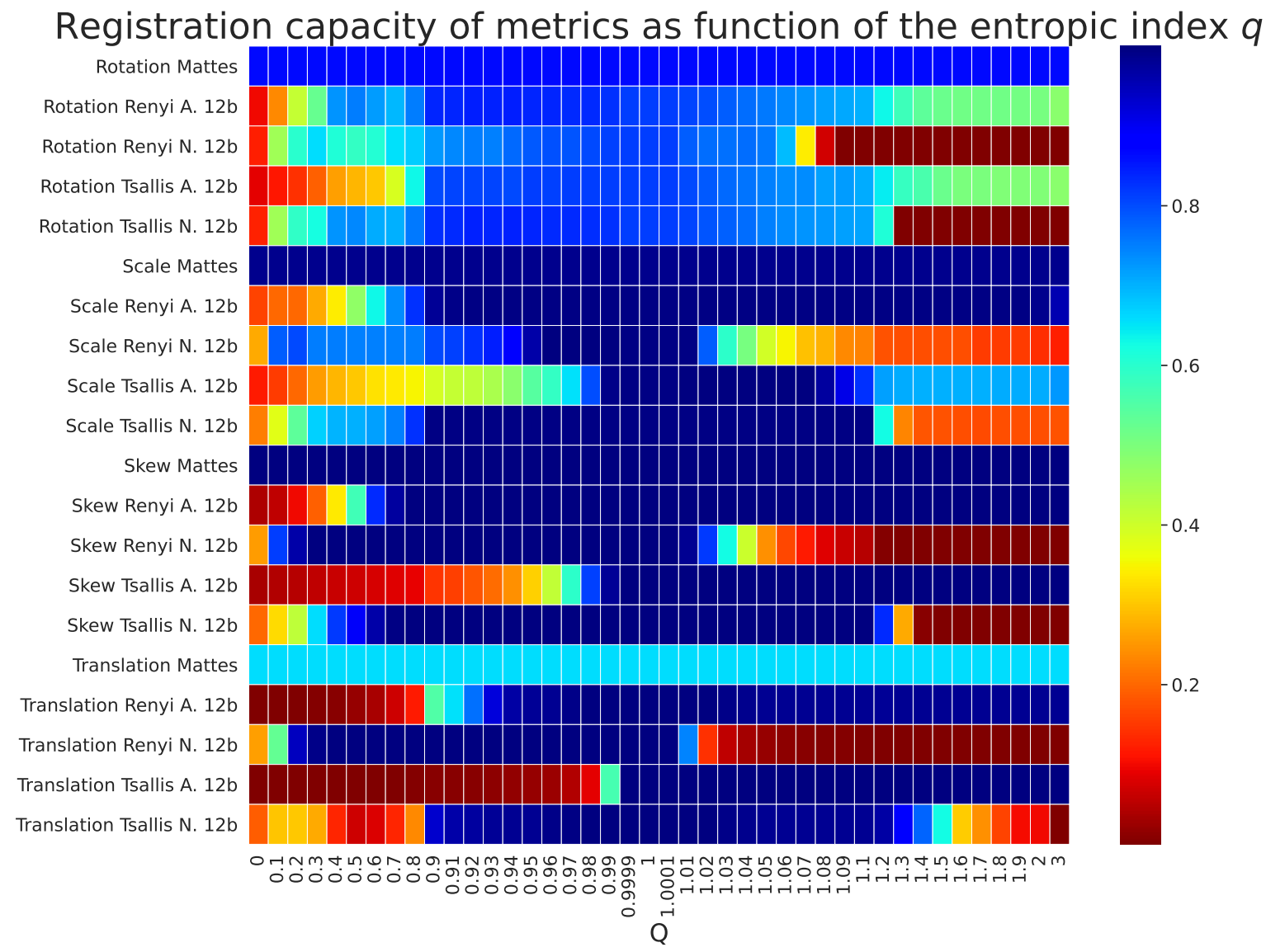

(b) Limited colorbar

Registration capacity of metrics as function of the entropic index $q$

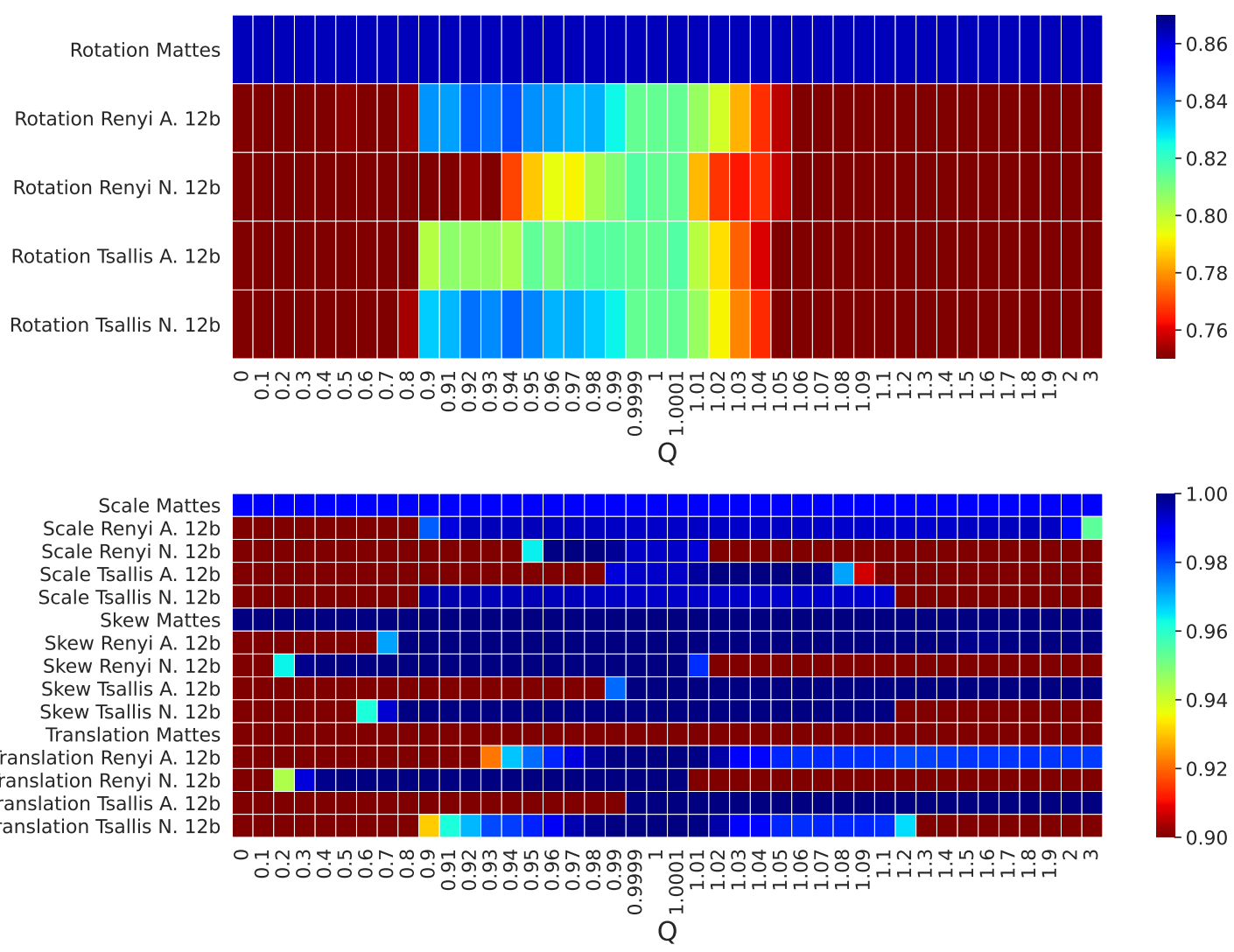

Source: author. 


\begin{tabular}{|c|c|c|c|c|c|c|c|c|c|c|c|c|c|c|c|c|c|c|c|c|}
\hline \multirow{3}{*}{$q$} & \multicolumn{5}{|c|}{ Rotation } & \multicolumn{5}{|c|}{ Scaling } & \multicolumn{5}{|c|}{ Skewness } & \multicolumn{5}{|c|}{ Translation } \\
\hline & \multirow{2}{*}{ Mattes } & \multicolumn{2}{|c|}{ Renyi } & \multicolumn{2}{|c|}{ Tsallis } & \multirow{2}{*}{ Mattes } & \multicolumn{2}{|c|}{ Renyi } & \multicolumn{2}{|c|}{ Tsallis } & \multirow{2}{*}{ Mattes } & \multicolumn{2}{|c|}{ Renyi } & \multicolumn{2}{|c|}{ Tsallis } & \multirow{2}{*}{ Mattes } & \multicolumn{2}{|c|}{ Renyi } & \multicolumn{2}{|c|}{ Tsallis } \\
\hline & & A. & $\mathrm{N}$. & A. & N. & & A. & N. & A. & N. & & A. & N. & A. & N. & & A. & N. & A. & N. \\
\hline 0 & 0.863 & 0.101 & 0.122 & 0.089 & 0.125 & 0.988 & 0.164 & 0.273 & 0.12 & 0.223 & 1.0 & 0.042 & 0.255 & 0.039 & 0.202 & 0.658 & 0.002 & 0.259 & 0.001 & 0.191 \\
\hline 0.1 & 0.863 & 0.236 & 0.455 & 0.115 & 0.455 & 0.988 & 0.201 & 0.785 & 0.155 & 0.376 & 1.0 & 0.056 & 0.813 & 0.043 & 0.321 & 0.658 & 0.002 & 0.53 & 0.001 & 0.299 \\
\hline 0.2 & 0.863 & 0.41 & 0.599 & 0.143 & 0.593 & 0.988 & 0.2 & 0.802 & 0.202 & 0.538 & 1.0 & 0.101 & 0.963 & 0.049 & 0.42 & 0.658 & 0.003 & 0.944 & 0.001 & 0.302 \\
\hline 0.3 & 0.863 & 0.523 & 0.658 & 0.195 & 0.623 & 0.988 & 0.271 & 0.75 & 0.255 & 0.669 & 1.0 & 0.194 & 0.999 & 0.058 & 0.658 & 0.658 & 0.008 & 0.992 & 0.001 & 0.271 \\
\hline 0.4 & 0.863 & 0.729 & 0.612 & 0.258 & 0.728 & 0.988 & 0.343 & 0.753 & 0.283 & 0.697 & 1.0 & 0.338 & 1.0 & 0.064 & 0.819 & 0.658 & 0.011 & 0.999 & 0.001 & 0.133 \\
\hline 0.5 & 0.863 & 0.752 & 0.588 & 0.283 & 0.74 & 0.988 & 0.473 & 0.748 & 0.304 & 0.701 & 1.0 & 0.573 & 1.0 & 0.069 & 0.894 & 0.658 & 0.022 & 1.0 & 0.002 & 0.067 \\
\hline 0.6 & 0.863 & 0.721 & 0.606 & 0.301 & 0.706 & 0.988 & 0.632 & 0.746 & 0.332 & 0.715 & 1.0 & 0.832 & 1.0 & 0.076 & 0.961 & 0.658 & 0.037 & 1.0 & 0.003 & 0.079 \\
\hline 0.7 & 0.863 & 0.695 & 0.654 & 0.391 & 0.703 & 0.988 & 0.737 & 0.747 & 0.336 & 0.749 & 1.0 & 0.971 & 1.0 & 0.083 & 0.993 & 0.658 & 0.068 & 1.0 & 0.003 & 0.13 \\
\hline 0.8 & 0.863 & 0.753 & 0.672 & 0.63 & 0.754 & 0.988 & 0.828 & 0.751 & 0.35 & 0.826 & 1.0 & 1.0 & 1.0 & 0.092 & 1.0 & 0.658 & 0.119 & 1.0 & 0.004 & 0.237 \\
\hline 0.9 & 0.863 & 0.837 & 0.725 & 0.802 & 0.831 & 0.988 & 0.978 & 0.801 & 0.392 & 0.996 & 1.0 & 1.0 & 1.0 & 0.147 & 1.0 & 0.658 & 0.552 & 1.0 & 0.008 & 0.93 \\
\hline 0.91 & 0.863 & 0.836 & 0.742 & 0.807 & 0.833 & 0.988 & 0.992 & 0.81 & 0.411 & 0.996 & 1.0 & 1.0 & 1.0 & 0.16 & 1.0 & 0.658 & 0.656 & 1.0 & 0.01 & 0.961 \\
\hline 0.92 & 0.863 & 0.845 & 0.752 & 0.806 & 0.842 & 0.988 & 0.994 & 0.825 & 0.422 & 0.995 & 1.0 & 1.0 & 1.0 & 0.181 & 1.0 & 0.658 & 0.766 & 1.0 & 0.011 & 0.969 \\
\hline 0.93 & 0.863 & 0.842 & 0.749 & 0.806 & 0.838 & 0.988 & 0.995 & 0.845 & 0.443 & 0.995 & 1.0 & 1.0 & 1.0 & 0.205 & 1.0 & 0.658 & 0.922 & 1.0 & 0.013 & 0.98 \\
\hline 0.94 & 0.863 & 0.846 & 0.77 & 0.804 & 0.843 & 0.988 & 0.994 & 0.876 & 0.482 & 0.995 & 1.0 & 1.0 & 1.0 & 0.245 & 1.0 & 0.658 & 0.968 & 1.0 & 0.016 & 0.981 \\
\hline 0.95 & 0.863 & 0.838 & 0.786 & 0.814 & 0.839 & 0.988 & 0.995 & 0.964 & 0.546 & 0.995 & 1.0 & 1.0 & 1.0 & 0.312 & 1.0 & 0.658 & 0.977 & 1.0 & 0.02 & 0.984 \\
\hline 0.96 & 0.863 & 0.836 & 0.794 & 0.809 & 0.834 & 0.988 & 0.994 & 0.999 & 0.588 & 0.995 & 1.0 & 1.0 & 1.0 & 0.415 & 1.0 & 0.658 & 0.984 & 1.0 & 0.026 & 0.99 \\
\hline 0.97 & 0.863 & 0.833 & 0.792 & 0.814 & 0.834 & 0.988 & 0.994 & 1.0 & 0.653 & 0.995 & 1.0 & 1.0 & 1.0 & 0.597 & 1.0 & 0.658 & 0.992 & 1.0 & 0.045 & 0.995 \\
\hline 0.98 & 0.863 & 0.834 & 0.804 & 0.815 & 0.831 & 0.988 & 0.994 & 1.0 & 0.799 & 0.995 & 1.0 & 1.0 & 1.0 & 0.812 & 1.0 & 0.658 & 0.998 & 1.0 & 0.086 & 0.998 \\
\hline 0.99 & 0.863 & 0.826 & 0.809 & 0.815 & 0.826 & 0.988 & 0.994 & 0.998 & 0.992 & 0.994 & 1.0 & 1.0 & 1.0 & 0.977 & 1.0 & 0.658 & 1.0 & 1.0 & 0.564 & 1.0 \\
\hline 0.9999 & 0.863 & 0.813 & 0.816 & 0.814 & 0.813 & 0.988 & 0.994 & 0.994 & 0.994 & 0.994 & 1.0 & 1.0 & 1.0 & 1.0 & 1.0 & 0.658 & 1.0 & 1.0 & 1.0 & 1.0 \\
\hline 1 & 0.863 & 0.814 & 0.814 & 0.814 & 0.814 & 0.988 & 0.994 & 0.994 & 0.994 & 0.994 & 1.0 & 1.0 & 1.0 & 1.0 & 1.0 & 0.658 & 1.0 & 1.0 & 1.0 & 1.0 \\
\hline
\end{tabular}

Table 2 - Metrics registration percentage as function of the entropic index $q$, limited in the range $q \leq 1$. (A. means additive, N. means non-additive) 


\begin{tabular}{|c|c|c|c|c|c|c|c|c|c|c|c|c|c|c|c|c|c|c|c|c|}
\hline \multirow{3}{*}{$q$} & \multicolumn{5}{|c|}{ Rotation } & \multicolumn{5}{|c|}{ Scaling } & \multicolumn{5}{|c|}{ Skewness } & \multicolumn{5}{|c|}{ Translation } \\
\hline & \multirow{2}{*}{ Mattes } & \multicolumn{2}{|c|}{ Renyi } & \multicolumn{2}{|c|}{ Tsallis } & \multirow{2}{*}{ Mattes } & \multicolumn{2}{|c|}{ Renyi } & \multicolumn{2}{|c|}{ Tsallis } & \multirow{2}{*}{ Mattes } & \multicolumn{2}{|c|}{ Renyi } & \multicolumn{2}{|c|}{ Tsallis } & \multirow{2}{*}{ Mattes } & \multicolumn{2}{|c|}{ Renyi } & \multicolumn{2}{|c|}{ Tsallis } \\
\hline & & A. & $\mathrm{N}$. & A. & $\mathrm{N}$. & & A. & $\mathrm{N}$. & A. & $\mathrm{N}$. & & A. & $\mathrm{N}$. & A. & $\mathrm{N}$. & & A. & $\mathrm{N}$. & A. & $\mathrm{N}$. \\
\hline 1 & 0.863 & 0.814 & 0.814 & 0.814 & 0.814 & 0.988 & 0.994 & 0.994 & 0.994 & 0.994 & 1.0 & 1.0 & 1.0 & 1.0 & 1.0 & 0.658 & 1.0 & 1.0 & 1.0 & 1.0 \\
\hline 1.0001 & 0.863 & 0.814 & 0.813 & 0.816 & 0.814 & 0.988 & 0.994 & 0.994 & 0.994 & 0.994 & 1.0 & 1.0 & 1.0 & 1.0 & 1.0 & 0.658 & 1.0 & 1.0 & 1.0 & 1.0 \\
\hline 1.01 & 0.863 & 0.806 & 0.784 & 0.801 & 0.806 & 0.988 & 0.994 & 0.992 & 0.997 & 0.994 & 1.0 & 1.0 & 0.983 & 1.0 & 1.0 & 0.658 & 0.999 & 0.742 & 1.0 & 0.999 \\
\hline 1.02 & 0.863 & 0.798 & 0.768 & 0.789 & 0.792 & 0.988 & 0.994 & 0.784 & 1.0 & 0.994 & 1.0 & 1.0 & 0.82 & 1.0 & 1.0 & 0.658 & 0.996 & 0.144 & 1.0 & 0.996 \\
\hline 1.03 & 0.863 & 0.783 & 0.764 & 0.773 & 0.778 & 0.988 & 0.994 & 0.597 & 1.0 & 0.993 & 1.0 & 1.0 & 0.627 & 1.0 & 1.0 & 0.658 & 0.989 & 0.055 & 1.0 & 0.989 \\
\hline 1.04 & 0.863 & 0.767 & 0.767 & 0.76 & 0.766 & 0.988 & 0.994 & 0.508 & 1.0 & 0.993 & 1.0 & 1.0 & 0.403 & 1.0 & 1.0 & 0.658 & 0.987 & 0.032 & 1.0 & 0.987 \\
\hline 1.05 & 0.863 & 0.756 & 0.757 & 0.748 & 0.748 & 0.988 & 0.994 & 0.398 & 1.0 & 0.993 & 1.0 & 1.0 & 0.246 & 1.0 & 1.0 & 0.658 & 0.984 & 0.021 & 1.0 & 0.984 \\
\hline 1.06 & 0.863 & 0.741 & 0.69 & 0.735 & 0.735 & 0.988 & 0.994 & 0.352 & 1.0 & 0.993 & 1.0 & 1.0 & 0.166 & 1.0 & 1.0 & 0.658 & 0.983 & 0.014 & 1.0 & 0.983 \\
\hline 1.07 & 0.863 & 0.73 & 0.343 & 0.736 & 0.727 & 0.988 & 0.994 & 0.295 & 0.998 & 0.993 & 1.0 & 1.0 & 0.117 & 1.0 & 1.0 & 0.658 & 0.983 & 0.009 & 1.0 & 0.984 \\
\hline 1.08 & 0.863 & 0.718 & 0.073 & 0.717 & 0.723 & 0.988 & 0.993 & 0.277 & 0.971 & 0.993 & 1.0 & 1.0 & 0.086 & 1.0 & 1.0 & 0.658 & 0.983 & 0.007 & 1.0 & 0.984 \\
\hline 1.09 & 0.863 & 0.708 & 0.001 & 0.721 & 0.718 & 0.988 & 0.993 & 0.237 & 0.907 & 0.993 & 1.0 & 1.0 & 0.064 & 1.0 & 1.0 & 0.658 & 0.983 & 0.006 & 1.0 & 0.984 \\
\hline 1.1 & 0.863 & 0.702 & 0.0 & 0.706 & 0.713 & 0.988 & 0.993 & 0.23 & 0.824 & 0.993 & 1.0 & 1.0 & 0.049 & 1.0 & 1.0 & 0.658 & 0.982 & 0.004 & 1.0 & 0.983 \\
\hline 1.2 & 0.863 & 0.629 & 0.0 & 0.643 & 0.61 & 0.988 & 0.993 & 0.178 & 0.717 & 0.626 & 1.0 & 1.0 & 0.006 & 1.0 & 0.832 & 0.658 & 0.98 & 0.001 & 1.0 & 0.966 \\
\hline 1.3 & 0.863 & 0.575 & 0.0 & 0.583 & 0.0 & 0.988 & 0.993 & 0.173 & 0.706 & 0.232 & 1.0 & 1.0 & 0.001 & 1.0 & 0.271 & 0.658 & 0.981 & 0.001 & 1.0 & 0.881 \\
\hline 1.4 & 0.863 & 0.537 & 0.0 & 0.56 & 0.0 & 0.988 & 0.993 & 0.172 & 0.702 & 0.183 & 1.0 & 1.0 & 0.0 & 1.0 & 0.008 & 0.658 & 0.982 & 0.001 & 1.0 & 0.775 \\
\hline 1.5 & 0.863 & 0.522 & 0.0 & 0.522 & 0.0 & 0.988 & 0.994 & 0.172 & 0.702 & 0.179 & 1.0 & 0.999 & 0.0 & 1.0 & 0.003 & 0.658 & 0.982 & 0.001 & 1.0 & 0.626 \\
\hline 1.6 & 0.863 & 0.513 & 0.0 & 0.502 & 0.0 & 0.988 & 0.994 & 0.172 & 0.701 & 0.172 & 1.0 & 0.999 & 0.0 & 1.0 & 0.002 & 0.658 & 0.982 & 0.001 & 1.0 & 0.306 \\
\hline 1.7 & 0.863 & 0.513 & 0.0 & 0.501 & 0.0 & 0.988 & 0.994 & 0.156 & 0.702 & 0.171 & 1.0 & 1.0 & 0.0 & 1.0 & 0.001 & 0.658 & 0.982 & 0.001 & 1.0 & 0.246 \\
\hline 1.8 & 0.863 & 0.514 & 0.0 & 0.493 & 0.0 & 0.988 & 0.994 & 0.156 & 0.704 & 0.174 & 1.0 & 1.0 & 0.0 & 1.0 & 0.001 & 0.658 & 0.983 & 0.0 & 1.0 & 0.162 \\
\hline 1.9 & 0.863 & 0.512 & 0.0 & 0.494 & 0.0 & 0.988 & 0.994 & 0.156 & 0.705 & 0.174 & 1.0 & 1.0 & 0.0 & 1.0 & 0.001 & 0.658 & 0.982 & 0.0 & 1.0 & 0.102 \\
\hline 2 & 0.863 & 0.507 & 0.0 & 0.496 & 0.0 & 0.988 & 0.987 & 0.14 & 0.707 & 0.174 & 1.0 & 1.0 & 0.0 & 1.0 & 0.001 & 0.658 & 0.982 & 0.0 & 1.0 & 0.101 \\
\hline 3 & 0.863 & 0.483 & 0.0 & 0.483 & 0.0 & 0.988 & 0.954 & 0.124 & 0.725 & 0.179 & 1.0 & 1.0 & 0.0 & 1.0 & 0.001 & 0.658 & 0.982 & 0.0 & 1.0 & 0.001 \\
\hline
\end{tabular}

Table 3 - Metrics registration percentage as function of the entropic index $q$, limited in the range $q \geq 1$. (A. mean additive, N. means non-additive) 


\subsection{Monte Carlo Results}

In this section, we will use scatter plots to visualize the Monte Carlo results, e.g., fig. 44, where each gray diamond shape represents a single registration result in the Monte Carlo experiment, with each Monte Carlo containing 1,000 or more results. The blue diamond shape and blue lines represent the statistical data, with the diamond being the mean value and the blue range representing the standard deviation of the results.

For the ITK Mattes metric, shown in fig. 44, for all categories of experiments listed in table 1, we have a mean near the $50 \mathrm{~mm}$ mark. Also, there are several outliers in the figure, as in the T2 experiments "Mattes HCP T2", and "Mattes HCP Cubic T2", where there is a denoted local minimum near the $75 \mathrm{~mm}$ mark.

Using the NAMIC/UNC MIDAS dataset, from sec. 3.2, an experiment with only single randomized T1 images $^{1}$ is shown in fig. 45, with exciting results. First, the ITK Mattes have lower final results than Shannon and Tsallis metrics. However, this is only valid for a fraction of the results. For the other part, the ITK Mattes registers to a wrong distance of more than $150 \mathrm{~mm}$ from the center. Both Mattes and Shannon present a significant number of outliers in the results. In contrast, Tsallis presents outliers but is limited to a low number, mostly less than five outliers in all the results. Another interesting result from this experiment is that the Tsallis metrics results degrade as we go into $q \geq 1.6$. This degradation may be connected to the smaller resolution found on those images compared to the HCP dataset.

Fig. 46 shows the results for fully randomized Monte Carlo experiments in the "T1×T2" experiments with fully randomized T1 fixed images and T2 moving images, with mostly different subjects ${ }^{2}$, and in the experiments without any T1 or T2 mention are the "T1×T1" fully randomized experiments. In all experiments, we can see Tsallis outperforming the Shannon metric. Tsallis presents few outliers or no outliers at all, while Shannon presents several outliers in the results. ITK Mattes presents several outliers in the fully randomized $\mathrm{T} 1 \times \mathrm{T} 2$, the most challenging scenario. Tsallis can successfully register, in these experiments, $\mathrm{T} 1 \times \mathrm{T} 1$ images within a $2 \mathrm{~mm}$ distance and $\mathrm{T} 1 \times \mathrm{T} 2$ within a $5 \mathrm{~mm}$ distance.

Table 4 shows the success rate of the similarity metrics depending on the acceptable final distance, i.e., the threshold for a successful or failed registration. Mattes have a poor performance in the randomized T2 scenario, even with an acceptable distance of $5 \mathrm{~mm}$. We obtained $5.05 \%$ success for Mattes, $56.50 \%$ for Shannon, and $99.75 \%$ for Tsallis, with $q=1.3$, and within the $5 \mathrm{~mm}$ distance threshold.

1 In this experiment we randomly draw a subject from the dataset and register the $\mathrm{T} 1$ image of the subject with itself, using the same images as fixed and moving images.

2 Since we randomly draw a subject for each fixed or moving images, there is a slight chance the subjects are the same. 
Table 4 - Monte Carlo results, means and deviations, in $\mathrm{mm}$. Acceptable distance percentage is the percentage of registrations that ended within this distance from the gold standard.

\begin{tabular}{|c|c|c|c|c|c|c|}
\hline \multirow[b]{2}{*}{ Method } & \multirow[b]{2}{*}{ Scenario } & \multirow[b]{2}{*}{ Mean } & \multirow[b]{2}{*}{ Deviation } & \multicolumn{3}{|c|}{ Acceptable distance (\%) } \\
\hline & & & & $1 \mathrm{~mm}$ & $3 \mathrm{~mm}$ & $5 \mathrm{~mm}$ \\
\hline Mattes & $\mathrm{T} 1$ & 48.08 & 72.24 & 64.70 & 65.80 & 66.00 \\
\hline Shannon Nearest & $\mathrm{T} 1$ & 0.68 & 5.38 & 97.04 & 99.61 & 99.62 \\
\hline Tsallis Nearest (1.7) & $\mathrm{T} 1$ & 0.48 & 3.31 & 99.33 & 99.97 & 99.97 \\
\hline Tsallis FastLanczos (1.2) & $\mathrm{T} 1$ & 0.39 & 0.15 & 98.90 & 100.00 & 100.00 \\
\hline Mattes & $\mathrm{T} 2$ & 55.14 & 72.01 & 58.15 & 58.25 & 58.25 \\
\hline Shannon Nearest & $\mathrm{T} 2$ & 1.48 & 8.69 & 97.20 & 99.00 & 99.10 \\
\hline Tsallis Nearest (1.3) & $\mathrm{T} 2$ & 0.45 & 0.10 & 99.90 & 100.00 & 100.00 \\
\hline Shannon Nearest & Randomized T1 & 1.62 & 10.88 & 94.09 & 98.21 & 98.26 \\
\hline Tsallis Nearest (1.3) & Randomized T1 & 0.63 & 6.04 & 98.87 & 99.91 & 99.91 \\
\hline Mattes & Randomized T2 & 45.25 & 56.94 & 0.10 & 1.10 & 5.05 \\
\hline Shannon Nearest & Randomized T2 & 7.46 & 16.81 & 2.10 & 37.15 & 56.50 \\
\hline Tsallis Nearest (1.3) & Randomized T2 & 1.51 & 0.71 & 20.85 & 95.80 & 99.75 \\
\hline
\end{tabular}

Source: author.

Fig. 47 shows results from the experiments using a parallel coordinates plot where the starting distances of each random sample starts on the left of the graphs, and the corresponding end distance, i.e., the final registration distances, ends on the right of the graphs. ITK Mattes presents several registrations that end in worse coordinates than starts, i.e., the registered image is worse than the initial image. These failures are shown by lines starting near the lower-left corner and ending near the top right corner in fig. 47a. Shannon presents some wrong registration, similar to Mattes, but to a lesser degree of magnitude, while Tsallis, on its best $q$ entropic index, present no wrong registration, with all registrations ending on the lower right corner. 
Figure 44 - Monte Carlo registration essays for Mattes
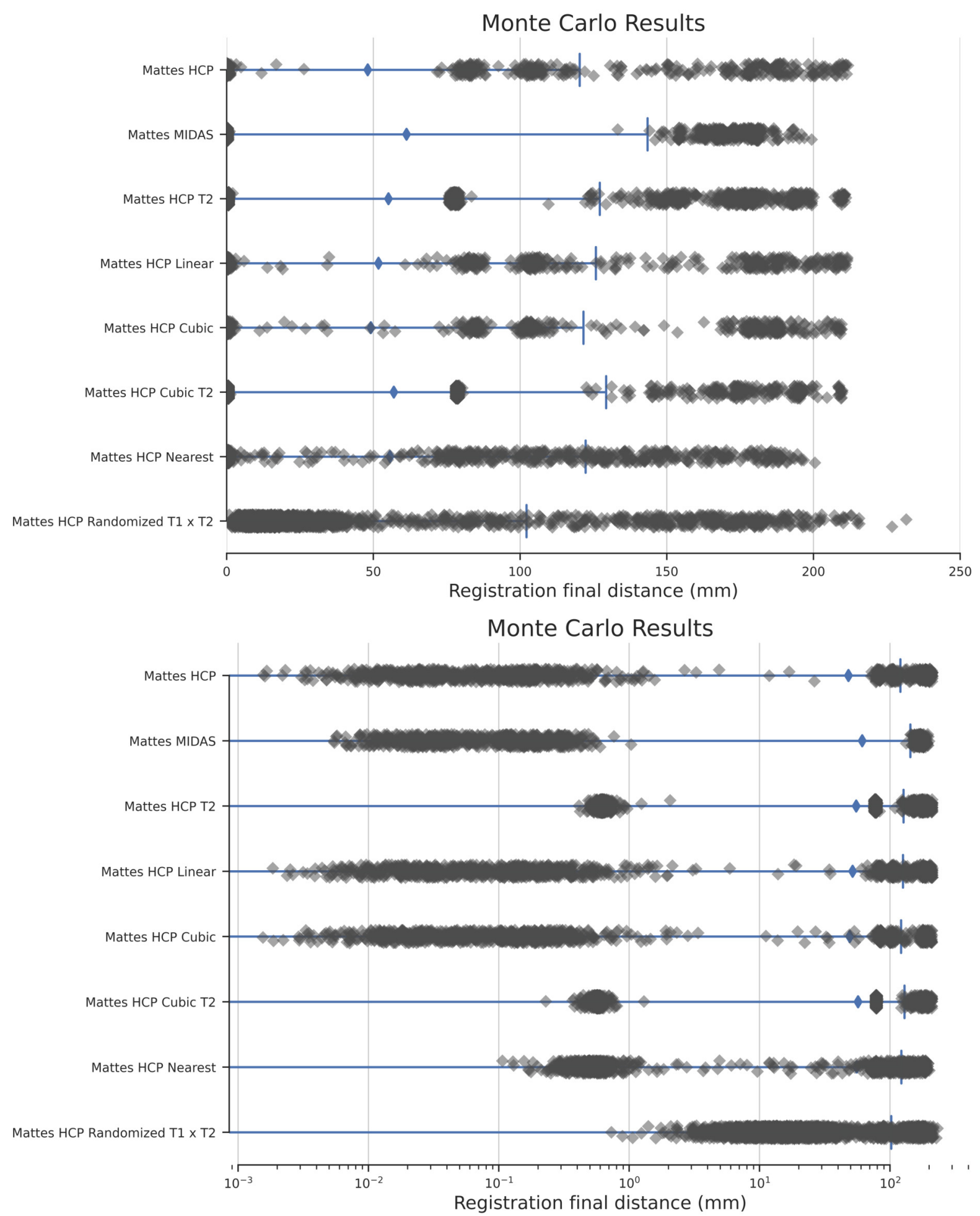

Monte Carlo registration essays for multiple metrics and scenarios. Each gray diamond represents a single registration result, the blue line is the standard deviation range $(68 \%)$ and the blue diamond is the mean distance of the final registration parameters, close to the left is better in this graph. Source: author. 
Figure 45 - Monte Carlo registration essays for UNC MIDAS
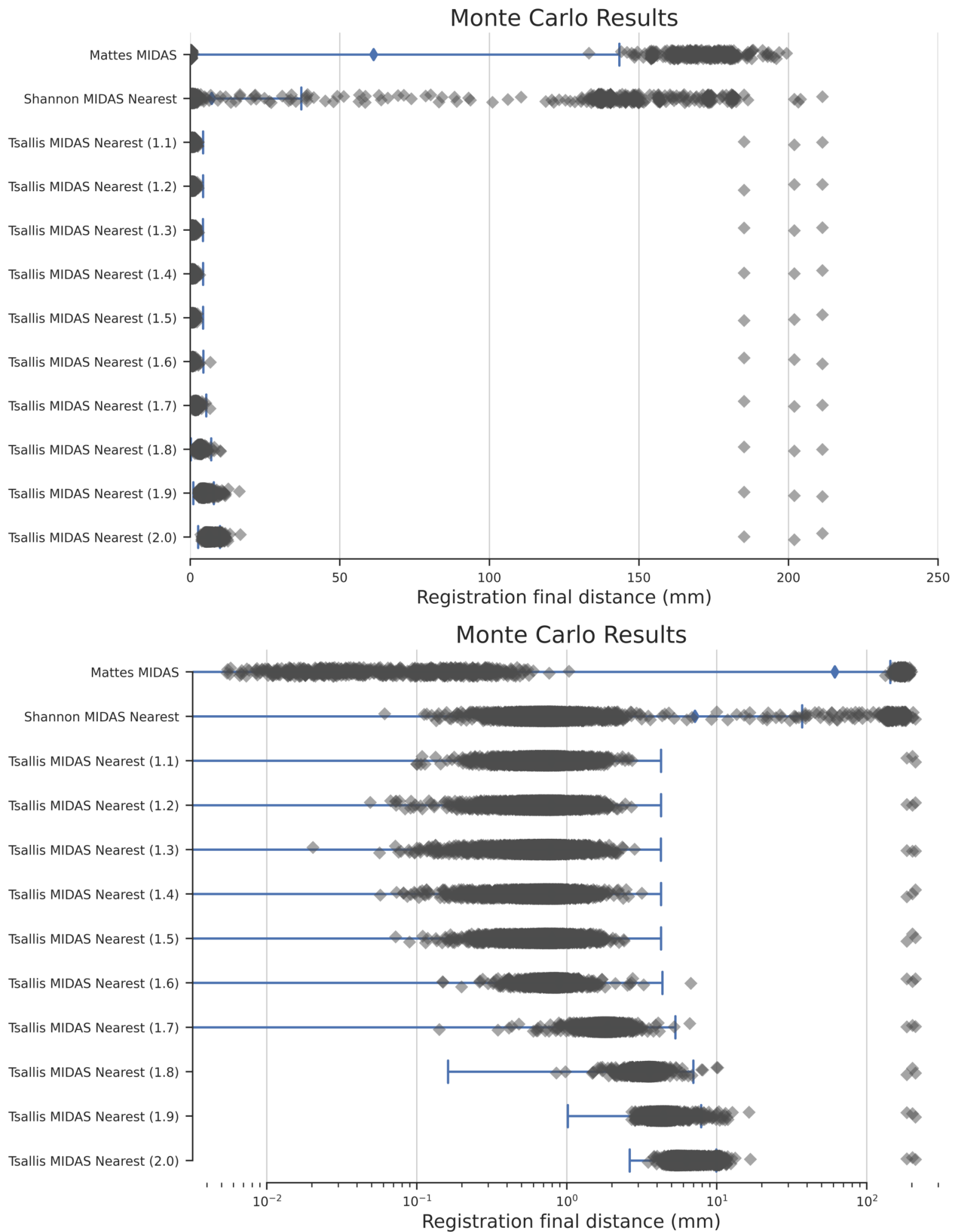

Monte Carlo registration essays for multiple metrics and scenarios. Each gray diamond represents a single registration result, the blue line is the standard deviation range $(68 \%)$ and the blue diamond is the mean distance of the final registration parameters, close to the left is better in this graph. Source: author. 
Figure 46 - Monte Carlo registration essays for Randomized test
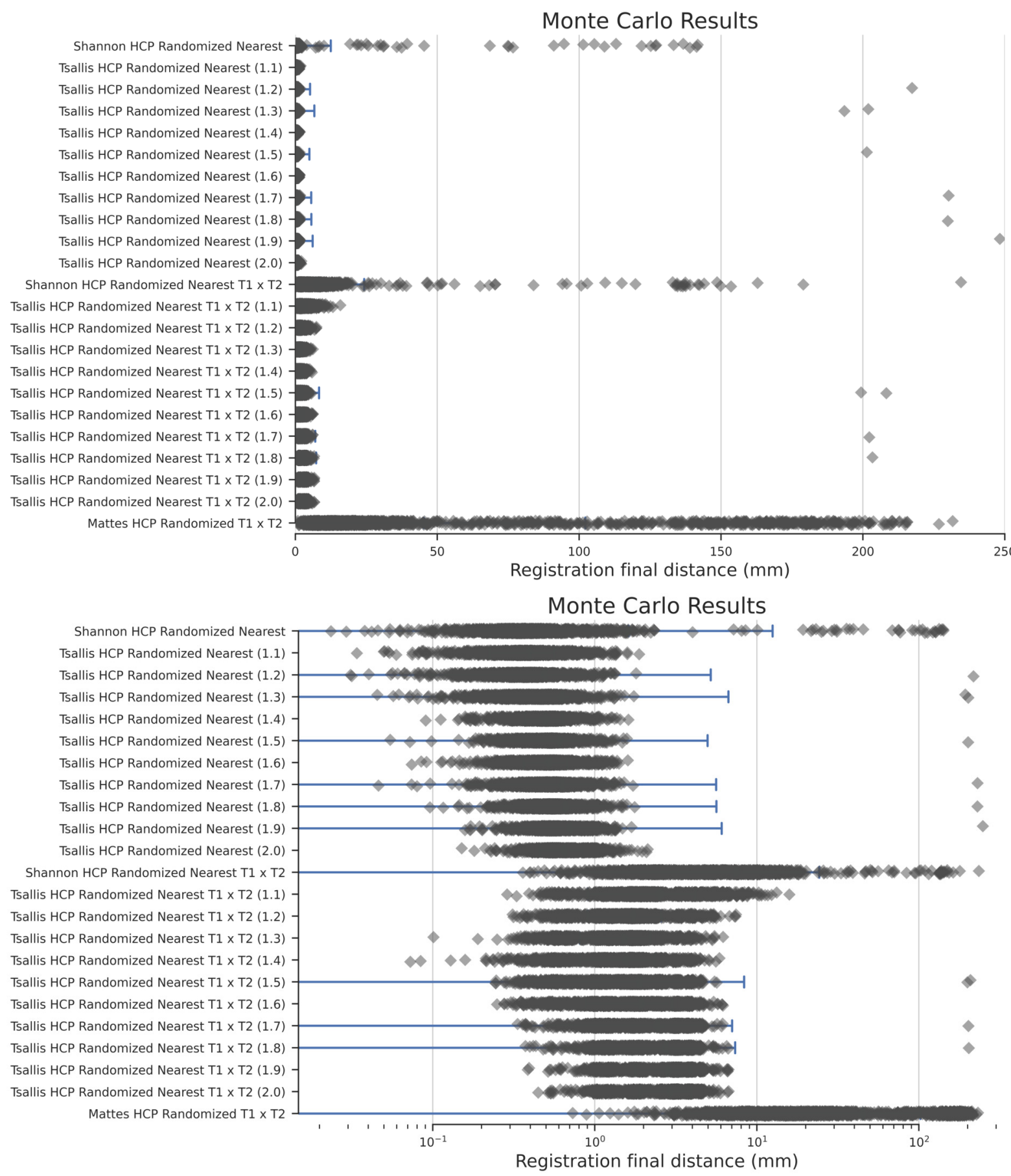

Monte Carlo registration essays for multiple metrics and scenarios. Each gray diamond represents a single registration result, the blue line is the standard deviation range (68\%) and the blue diamond is the mean distance of the final registration parameters, close to the left is better in this graph. Source: author. 
Figure 47 - Monte Carlo registration essays as parallel coordinates
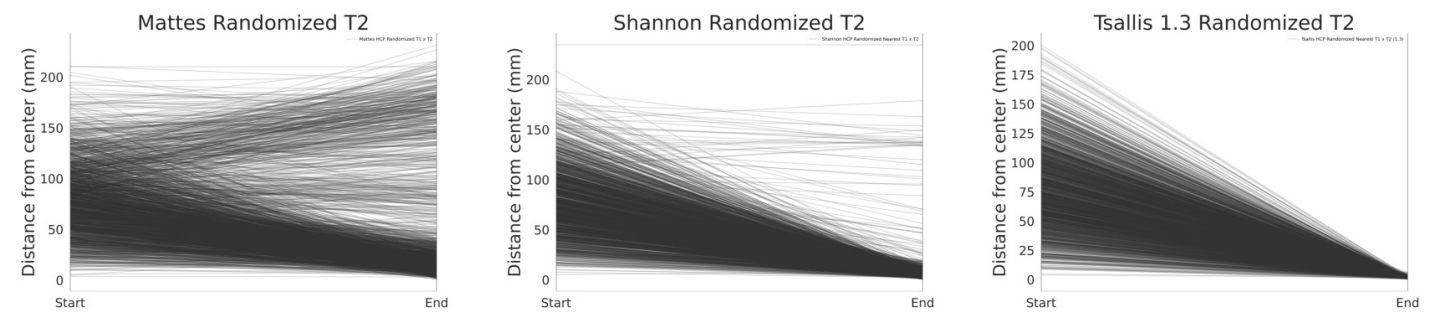

Monte Carlo registration essays as parallel coordinates, each line represents a registration essay, start is the starting distance from the gold standard and end is the final distance achieved, an ideal registration will come from anywhere on the left and end on the right bottom where the gold standard is on this graph. Source: author. 


\section{Conclusion}

\subsection{Discussion}

The proposed hypothesis is:

\section{"The use of generalized entropies on mutual information metrics can improve affine registration of brain images."}

There is an argument for the roundness of isosurfaces leading to better gradient paths that flows more directly to the center. However, there is subjectiveness in determining how an isosurface is better than another, by only using a visualization technique. Therefore, we consider the isosurfaces images as weak evidence for the hypothesis and not enough, by itself, to validate and support the hypothesis.

The registration capacity measurement, as provided by the naive algorithm defined in sec. 3.3.3, provides an objective analysis that allows a quantitative comparison between metrics. Therefore, the evidence collected by those experiments can be summarized as follows:

Translation presents a full registration capacity just using Shannon entropy without any histogram binning. However, when histogram binning is used, Shannon registration capacity degrades. At the same time, Tsallis can be tuned to achieve a full registration capacity by using the Tsallis additive metrics, or very near it, in the cases of 2 bits binning. Using 1 bit binning seems to break translation registration space, but we can still achieve higher levels than Mattes and Shannon with Tsallis. Moreover, Tsallis registration remains stable for changes in the $q>1$ range, allowing us to use a wide range of entropic indexes.

Rotation is very sensible to histogram binning and seems to demand it to achieve good registration capacity. Unfortunately, no metric tested could achieve a full or near full registration capacity. However, Tsallis metrics achieved some gain over the Mattes and Shannon metrics, using the Tsallis non-additive metrics and histogram binning with 2 and 4 bits.

Scaling presents a near full registration capacity using Mattes and Shannon entropy. This transformation also benefits from histogram binning with some superiority using 4 bits binning. Tsallis can improve the Shannon level in the $q>1$ range. However, the tuning of the entropic index is very demanding, with the best results appearing on the $1 \leq q \leq 1.1$ range with Tsallis additive metrics. The exact entropic indexes 
where we have the maximum registration capacity depend on the histogram binning, showing a slight instability where small changes in the $q$ value can significantly degrade the registration capacity. There is some stability over the $q>1$ range for non-additive Tsallis metrics with binning bits $\leq 6$, but the overall capacity is less than the fine-tuned additive Tsallis metrics.

Skewness presents the best overall results and full capacity for all metrics tested. So, for example, the Shannon level, $q \rightarrow 1$, can be used in the Tsallis metrics, or we can choose some $q$ value very near it. While Tsallis non-additive metrics present more stability in the $q<1$ range, Tsallis additive metrics are more stable in the $q>1$ range. Full capacity metric on this transformation is quickly achieved with various binning settings in the range $1 \leq q \leq 1.1$.

The Monte Carlo experiment, sec. 3.4 and sec. 4.5 provides more robust evidence for registration capacity. The major argument for the weakness of the registration capacity simulation is on the few subjects analyzed by it, mostly due to the time-consuming task of generating the full metric output image for each subject set. Monte Carlo evidence's are as follows:

$\mathbf{T} 1 \times \mathbf{T} 1$, same subject shows some challenges on the ITK Mattes metric, with only $64.7 \%$ registration within the $1 \mathrm{~mm}$ distance, with Shannon achieving $97.0 \%$ and Tsallis outperforming both with $99.3 \%$ capacity. If we allow some error on the registration, e.g., using a "coarse" registration stage for a later "fine" registration, we have in the $5 \mathrm{~mm}$ distance Mattes with $66.00 \%$, Shannon with $99.62 \%$ and Tsallis with $99.97 \%$ registration capacity.

T1 $\times \mathbf{T} 2$, same subject present more challenges on both ITK Mattes and Shannon metrics, with Mattes having $58.25 \%$ capacity with $5 \mathrm{~mm}$ acceptable distance, Shannon having $97.20 \%$ for $1 \mathrm{~mm}$ distance, and $99.10 \%$ for $5 \mathrm{~mm}$ distance. Tsallis achieved $99.90 \%$ registration within $1 \mathrm{~mm}$ and $100.0 \%$ within $3 \mathrm{~mm}$.

$\mathbf{T} 1 \times \mathbf{T} 1$, different subjects presents Shannon with $94.09 \%$ within $1 \mathrm{~mm}$ while Tsallis has $98.87 \%$. For the $5 \mathrm{~mm}$ distance, Shannon has $98.26 \%$ while Tsallis has $99.91 \%$ registration capacity. Mattes was not tested in this scenario.

T1 $\times \mathbf{T} 2$, different subjects is the most challenging scenario, resulting in very poor Mattes results, with only $5.05 \%$ capacity within $5 \mathrm{~mm}$ distance, while Shannon has $56.50 \%$ and Tsallis has $99.75 \%$ capacity. For the $1 \mathrm{~mm}$ distance, Mattes has $0.1 \%$, Shannon has $2.10 \%$, and Tsallis has $20.85 \%$ registration capacity.

Tsallis could outperform both Shannon and ITK Mattes metrics for the translation transformation in all Monte Carlo scenarios. This corroborates the results on sec. 4.4, 
which can be compared to the "T1×T1, same subject" scenario, where Mattes had $65.8 \%$ capacity and both Shannon and Tsallis had 100\% capacity, while in the Monte Carlo, we have Mattes with 65,80\% within the $3 \mathrm{~mm}$ distance, Shannon with $99.61 \%$ and Tsallis with $99.97 \%$, showing how useful the naive algorithm is to predict the registration capacity.

One of the strongest arguments for the MI use in image registration is its robustness in inter-subjects and inter-modality registrations. In our experiments, the Shannon MI metric showed robustness for inter-subject registration, as the "T1 $1 \times \mathrm{T} 1$, different subject" result was high, with $94-98 \%$ registration and inter-modality registration, as the "T1 $\times \mathrm{T} 2$, same subject" was also high, with $97-99 \%$ registration. However, as we checked with both inter-subject and inter-modality registration, the classical Shannon MI suffers and degrades, as the "T $1 \times \mathrm{T} 2$, different subject" result was low, with $2-56 \%$ registration. We have the highest gain in this challenging scenario using Tsallis metrics, with results between $20-99 \%$ registration.

The argument on Tsallis' robustness for both inter-subject and inter-modality registration from the Monte Carlo experiments is very clear and strong. Also, note that in all other scenarios, we also gain by using Tsallis metrics.

Seems to be no disadvantages to using Tsallis' entropy, as in the worst case, it can be used with $q \rightarrow 1$, resulting in Shannon's entropy with the same code. In our case, we tested with $q=0.9999$ and $q=1.0001$ with very near results to the Shannon results. Only in the 1-bit histogram binning, we note a noticeable difference between the two entropies. The computational complexity of both equations is pretty similar since Shannon uses the $p(i) \times \log p(i)$ kernel, and Tsallis uses the $p(i)^{q}$ kernel, with the kernel being the part inside the summation and the most impactful one on the computational time.

To compute the $p(i)^{q}$ kernel, we use the pow function of the programming language. This function is normally converted as:

$$
\begin{aligned}
a^{b} & =e^{b \times \log a} \\
\operatorname{pow}(\mathrm{a}, \mathrm{b}) & =\exp \left(\mathrm{b}^{*} \log (\mathrm{a})\right)
\end{aligned}
$$

This means that, in the kernel, Tsallis has almost double the operations, it has an exp, a log and multiplication, while Shannon has the log and a multiplication.

However, in our experiments, the most computing-demanding part of the registration process is the geometric transformation, primarily due to the image sizes causing too many cache losses. After all the transformation and histogram generation, we have small histogram vectors used in the Shannon or Tsallis equations. The extra computing time needed for Tsallis calculations is negligible compared to those extra steps like transformation and interpolation. Also, there is a resource of using Tsallis with $q=2$, where its 
kernel becomes $p(i)^{2}=p(i) * p(i)$, using a single multiplication to calculate it. However, this gain will be negligible in contrast with the other stages of the registration process.

To achieve the best possible registration capacity, we will need to use Tsallis in a "meta-metric" style. Different entropic indexes $q$, and additivity settings, with additive or non-additive Tsallis, depending on what parameter is optimized, are needed to register successfully. Also, to improve rotation registration, we will need to use histogram binning in this "meta-metric" function.

\subsection{Meta-metric selection}

We try to synthesize all data collected in sec. 4.3 in the fig. 48 , where we can see the registration capacity of all metrics tried, with all different histogram binning used, in the scaling of $0-100 \%$. In translation, scaling, and skewness, we can achieve the maximum in all binning settings. However, in rotation, we need to stay in the $2-4$ histogram bins with either Renyi additive or Tsallis non-additive.

To better understand this registration, the capacity space is plotted in fig. 49 with the colorbar limited between the maximum value possible for each transformation and $97 \%$ of this maximum value. The final and condensed analysis of this data is, for each transformation:

Translation registration is possible for the entire histogram binning only by using Tsallis additive, with the 2 bits binning being marginally worse than the others. Shannon outperforms ITK Mattes in all binning except for 2 bits binning.

Rotation is best achieved using 4 bits histogram binning, with a slightly worse result using 2 bits, both using the additive versions of Tsallis and Renyi MI. Shannon only reaches ITK Mattes performance when using $2-4$ bits in binning.

Scaling registration is possible for the entire histogram binning only by using Tsallis additive, with fine-tuning of the $q$ entropic index depending on the binning used. Shannon outperforms ITK Mattes in all binning except for 2 bits binning.

Skewness registration is possible for the entire histogram binning using Mattes, Renyi, or Tsallis. Shannon reaches ITK Mattes performance in all binning with exception to 2 bits binning.

The best options for the meta-metric settings are defined in table 5 . The 4 bits histogram binning can be used in all transformations, remaining only the need to change the additivity depending on the parameter's dimension. A single entropic index of $q=1.3$ can be used with success over all the transformations. However, it is best to fine-tune these settings for different needs than brain image registration. 
Table $\mathbf{5}$ - Proposed meta-metric settings for affine registration.

\begin{tabular}{lllll}
\hline Transformation type & Metric family & Additivity & Histogram Binning & Entropic Index $q$ \\
\hline Translation & Tsallis & Additive & 4 & $1.1 \leq q \leq 2.0$ \\
Rotation & Tsallis & Nonadditive & 4 & 1.3 \\
Scaling & Tsallis & Nonadditive & 4 & $1.1 \leq q \leq 2.0$ \\
Skewness & Tsallis & Nonadditive & 4 & $1.1 \leq q \leq 2.0$ \\
\hline
\end{tabular}

Source: author.

Figure 48 - Metrics registration capacity with different histogram binning settings

Registration capacity of metrics using Translation

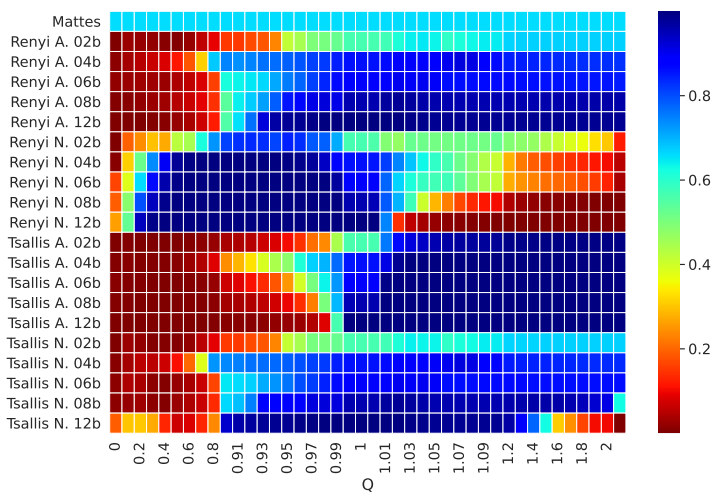

Registration capacity of metrics using Scale

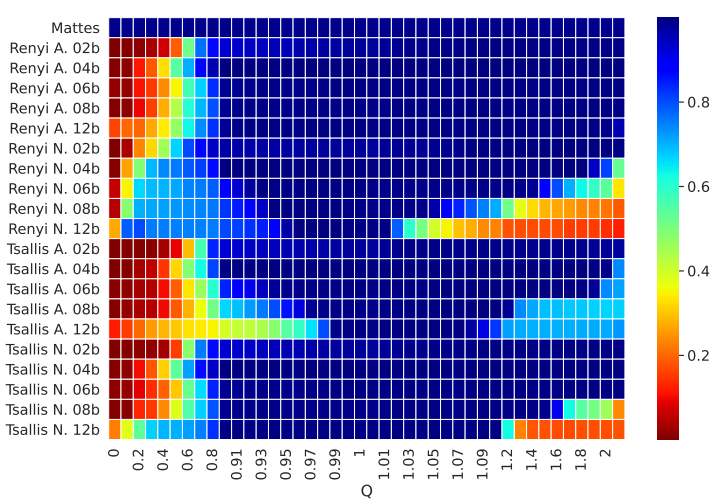

Registration capacity of metrics using Rotation

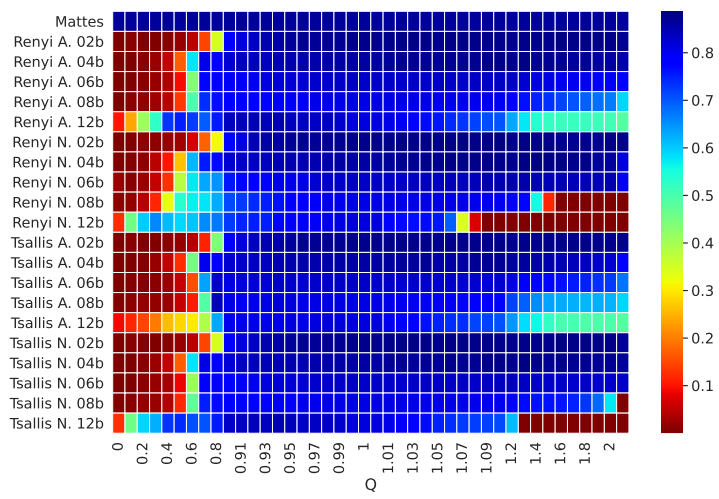

Registration capacity of metrics using Skew

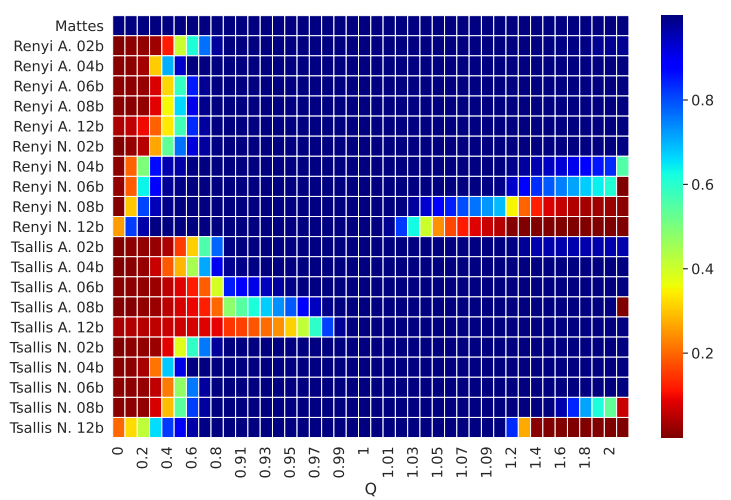

Source: author. 
Figure 49 - Metrics registration capacity with different histogram binning settings and colorbar limited

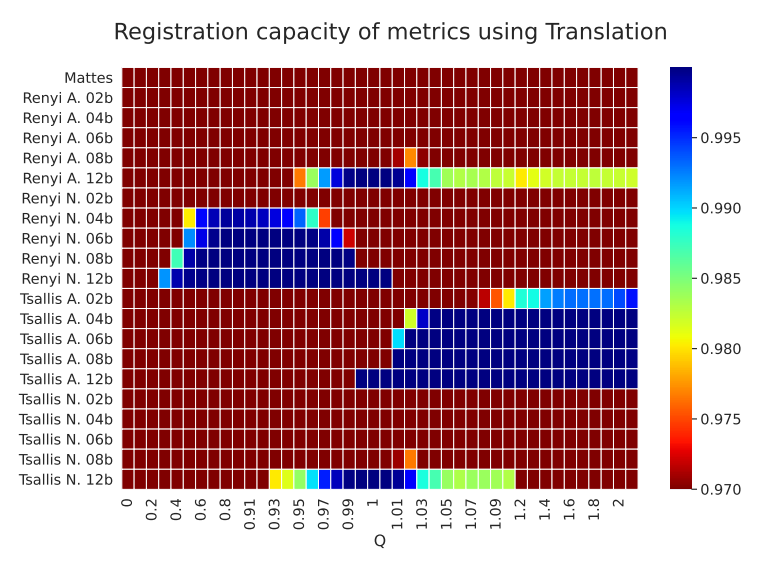

Registration capacity of metrics using Scale

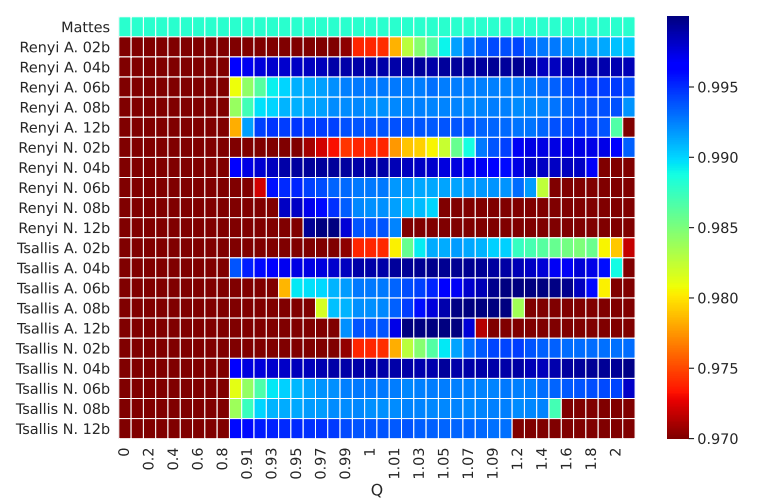

Registration capacity of metrics using Rotation

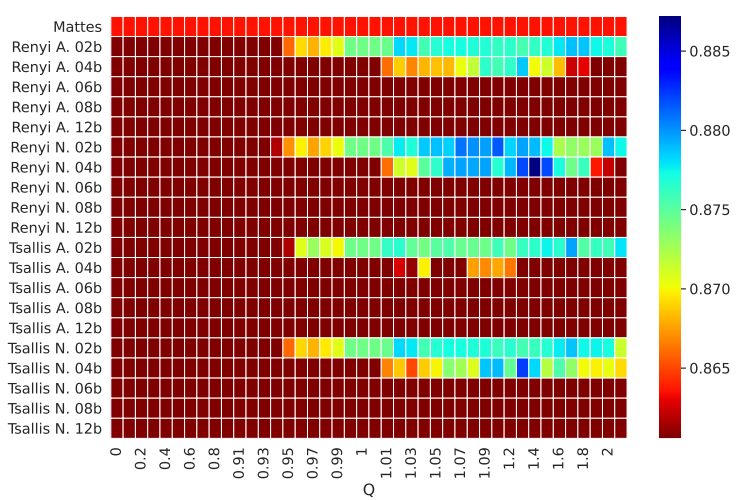

Registration capacity of metrics using Skew

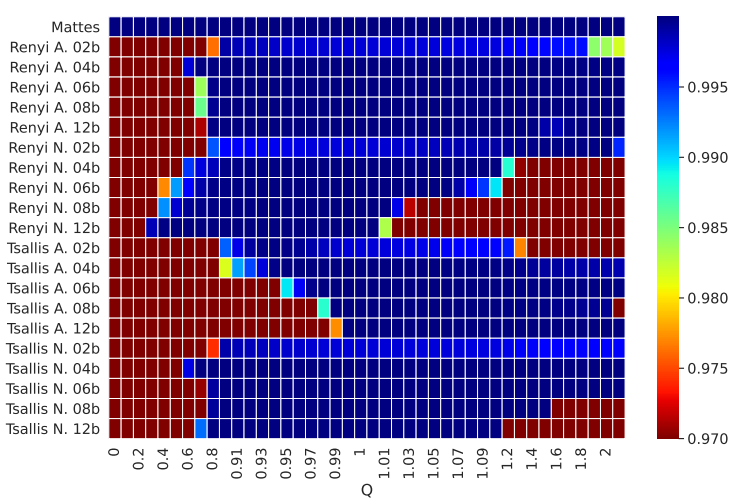

Metrics registration capacity with different histogram binning settings with colorbar limited to $97-100 \%$ of the best possible capacity. Source: author.

\subsubsection{Affine registration example}

Although our focus is an empirical and objective analysis of GMI, there is a need and interest in actual registration results. An example of affine registration using the meta-metric can be seen in figure 50, where Tsallis provides a different registration result than ITK Mattes and Shannon. This difference can be helpful or not, depending on the medical application and later registration stages, e.g., a deformable spline registration.

\subsection{Conclusion}

About the proposed hypothesis:

"The use of generalized entropies on mutual information metrics can improve affine registration of brain images."

The evidence collected in the prior sections strongly supports our hypothesis as valid, allowing the conclusion that Tsallis' entropy can improve brain images registration, especially the improvement is more significant in the inter-subject and inter-modality 
Figure 50 - Example of affine registration using the meta-metric

(a) HCP \#172635

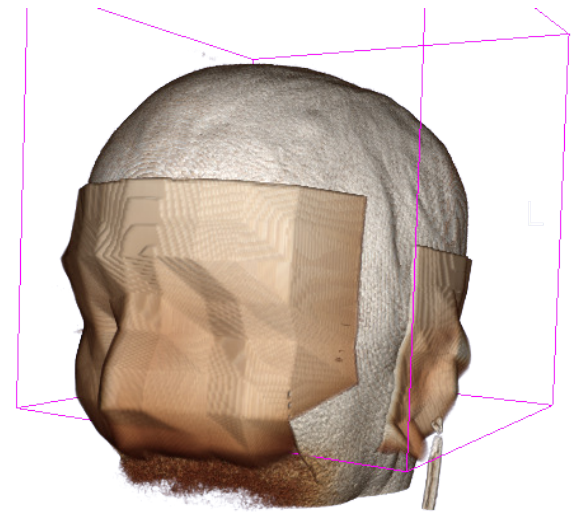

(c) ITK Mattes

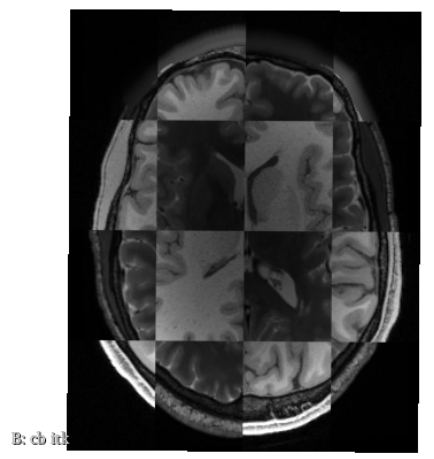

(b) HCP \#901038

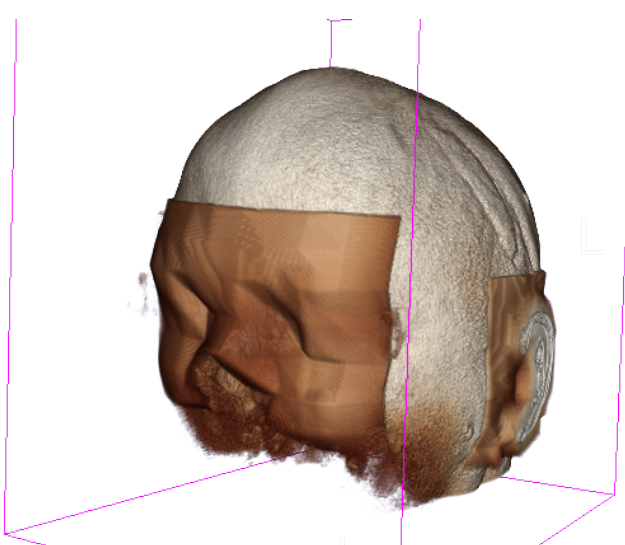

(e) Tsallis (d) Shannon

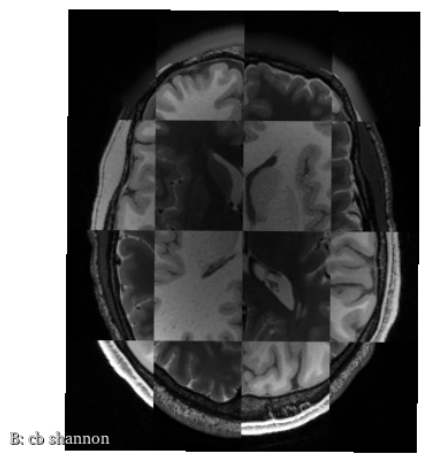

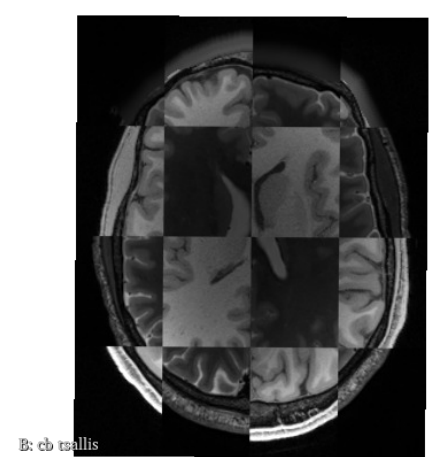

Affine registration between HCP subject 172635 T1 MRI and 901038 T2 MRI, using ITK Mattes, Shannon and Tsallis. 3D renders of subjects (top) and checkboard axial views from moving and registered images (bottom). Source: author.

scenarios.

\subsubsection{Future work}

There is still room for improvement on the rotation transformation since the metrics analyzed in this study only achieve $88.2 \%$ for the Tsallis non-additive with $q=1.3$ and $88.7 \%$ for the Renyi non-additive with $q=1.4$, both with 4 bits histogram binning. Further studies on this transformation can focus on how the rotation matrix is built, as different parameters can be used, e.g., degrees, versors, and quaternions.

As proposed in this study, the creation of a "meta-metric" demands changes to other structures in the image registration process, such as the optimizer block, to handle different metrics for each dimension in the parameter space.

This study relies on the premise that we can separate the parameter space, by transformation type, with no interdependence between those dimensions. This approach is used by other registration software that starts with low dimensionality, e.g., only doing translation registration, and grows the parameters space incorporating other transfor- 
mations in new stages, e.g., a second stage with translation and rotation and the third stage with translation, rotation, and scaling. Further study can be made on how this premise holds in reality and what visualization techniques can be used to analyze more than three dimensions at a time, e.g., maybe using techniques like principal component analysis (PCA) to reduce a 6-dimensional space with translation and rotation to a virtual 3-dimensional space that can be analyzed with the techniques here presented, e.g., using isosurfaces to understand this PCA space.

This study focuses on an automated approach for registration, demanding metrics that present a wide capture range. Another study can be made to understand how to improve the registration scheme on a 2-stages approach, with this "meta-metric" as a coarse registration stage and another metric serving as a fine registration stage. This fine stage metric does not demand a wide capture range. It should focus on final registration accuracy in order to fine register the image initially registered within this metric capture range, e.g., a coarse translation to register the image within $5 \mathrm{~mm}$ of the gold standard and a final translation to fine-tune this registration as near as possible to the gold standard. 


\section{Bibliography}

ALEMANY, n.; ZANETTE, n. Fractal random walks from a variational formalism for Tsallis entropies. Physical Review. E, Statistical Physics, Plasmas, Fluids, and Related Interdisciplinary Topics, v. 49, n. 2, p. R956-R958, fev. 1994. ISSN 1063-651X. Cited on page 64.

BERGSTRöM, M. et al. Head Fixation Device for Reproducible Position Alignment in Transmission CT and Positron Emission Tomography. Journal of Computer Assisted Tomography, v. 5, n. 1, p. 136-141, fev. 1981. ISSN 0363-8715. Disponível em: <https://journals.lww.com/jcat/Abstract/1981/02000/Head_Fixation_Device_for_ Reproducible_Position.30.aspx>. Cited 2 times on pages 43 and 44 .

BIBLIOTHECAE novissimae observationum ac recensionum sectio V. Sumptu Novi Bibliopoli, 1718. Google-Books-ID: SmUTAAAAQAAJ. Disponível em: $<$ http://digitale.bibliothek.uni-halle.de/vd18/content/pageview/4921254>. Cited on page 28.

BILINEAR interpolation. 2021. Page Version ID: 1014129604. Disponível em: < https: //en.wikipedia.org/w/index.php?title=Bilinear_interpolation\&oldid $=1014129604>$. Cited on page 52 .

BORLAND, L.; PLASTINO, A. R.; TSALLIS, C. Information gain within nonextensive thermostatistics. Journal of Mathematical Physics, v. 39, n. 12, p. 6490-6501, 1998. Disponível em: <https://doi.org/10.1063/1.532660>. Cited on page 65.

BROWN, L. G. A survey of image registration techniques. ACM Comput. Surv., Association for Computing Machinery, New York, NY, USA, v. 24, n. 4, p. 325-376, dez. 1992. ISSN 0360-0300. Disponível em: <https://doi.org/10.1145/146370.146374>. Cited on page 41.

CAMERA obscura. 2021. Page Version ID: 1010773140. Disponível em: < https: //en.wikipedia.org/w/index.php?title=Camera_obscura\&oldid=1010773140>. Cited on page 28.

CHEAH, T. C.; SHANMUGAM, S. A.; MANN, K. A. L. Medical image registration by maximizing mutual information based on combination of intensity and gradient information. In: 2012 International Conference on Biomedical Engineering (ICoBE). [S.l.]: IEEE, 2012. p. 368-372. Cited on page 58.

CHUANG, K.-S.; VAlENTINO, D. J.; HUANG, H. K. Measurement of fractal dimension using 3-D technique. In: Medical Imaging V: Image Processing. International Society for Optics and Photonics, 1991. v. 1445, p. 341-347. Disponível em: <https://www.spiedigitallibrary.org/conference-proceedings-of-spie/1445/0000/ Measurement-of-fractal-dimension-using-3-D-technique/10.1117/12.45231.short>. Cited on page 70 .

COLLIGNON, A. et al. Automated multi-modality image registration based on information theory. In: Bizais, 1995. Cited on page 57. 
COLLIGNON, A. et al. 3D Multi-Modality Medical Image Registration Using Feature Space Clustering. In: AYACHE, N. (Ed.). Computer Vision, Virtual Reality and Robotics in Medicine. Berlin, Heidelberg: Springer, 1995. (Lecture Notes in Computer Science), p. 195-204. ISBN 9783540491972. Cited 2 times on pages 56 and 75.

CORMACK, A. M. Representation of a Function by Its Line Integrals, with Some Radiological Applications. Journal of Applied Physics, American Institute of Physics, v. 34, n. 9, p. 2722-2727, set. 1963. ISSN 0021-8979. Disponível em: $<$ https://aip.scitation.org/doi/10.1063/1.1729798>. Cited on page 28.

EULER, L. Euler's rotation theorem. Disponível em: < http://en.wikipedia.org/wiki/ Euler's_rotation_theorem>. Cited on page 47.

EULER, L. Generalae formulas. Novi Commentarii academiae scientiarum Petropolitanae, 1776. Disponível em: <http://www.17centurymaths.com/contents/euler/e478tr.pdf>. Cited on page 47.

FREE, S. L. et al. Three-Dimensional Fractal Analysis of the White Matter Surface from Magnetic Resonance Images of the Human Brain. Cerebral Cortex, v. 6, n. 6, p. 830-836, nov. 1996. ISSN 1047-3211. Disponível em: <https://doi.org/10.1093/cercor/6.6.830>. Cited on page 70.

FURUICHI, S. Information theoretical properties of Tsallis entropies. Journal of Mathematical Physics, American Institute of Physics, v. 47, n. 2, p. 023302, fev. 2006. ISSN 0022-2488. Disponível em: < https://aip-scitation-org.ez67.periodicos.capes.gov.br/ doi/full/10.1063/1.2165744>. Cited on page 65 .

GEOMETRIC transformation. 2021. Page Version ID: 1001079671. Disponível em: < https: //en.wikipedia.org/w/index.php?title=Geometric_transformation\&oldid $=1001079671>$. Cited on page 45 .

GLASSER, M. F. et al. The minimal preprocessing pipelines for the Human Connectome Project. NeuroImage, Academic Press, v. 80, p. 105-124, oct 2013. ISSN 1053-8119.

Disponível em: < https://www.sciencedirect.com/science/article/pii/S1053811913005053>. Cited on page 75 .

HOFMAN, M. A. The fractal geometry of convoluted brains. Journal Fur Hirnforschung, v. 32, n. 1, p. 103-111, 1991. ISSN 0021-8359. Cited on page 70.

HOULT, D. I.; BHAKAR, B. NMR signal reception: Virtual photons and coherent spontaneous emission. Concepts in Magnetic Resonance, v. 9, n. 5, p. 277-297, 1997. ISSN 1099-0534. Disponível em: <https://onlinelibrary.wiley.com/doi/abs/10.1002/\%28SICI\% 291099-0534\%281997\%299\%3A5\%3C277\%3A\%3AAID-CMR1\%3E3.0.CO\%3B2-W>. Cited on page 28.

HOUNSFIELD, G. N. Computerized transverse axial scanning (tomography): Part 1. Description of system. The British Journal of Radiology, The British Institute of Radiology, jan. 2014. Disponível em: < https://www.birpublications.org/doi/abs/10.1259/ 0007-1285-46-552-1016>. Cited on page 28.

IMAGE registration. 2021. Page Version ID: 1014114384. Disponível em: < https: //en.wikipedia.org/w/index.php?title $=$ Image_registration\&oldid $=1014114384>$. Cited on page 40. 
JAMES, I. Claude Elwood Shannon 30 April 1916 - 24 February 2001. Biographical Memoirs of Fellows of the Royal Society, Royal Society, v. 55, p. 257-265, dez. 2009. Disponível em: <https://royalsocietypublishing.org/doi/10.1098/rsbm.2009.0015>. Cited on page 34 .

JERISON, H. J. Evolution of the Brain and Intelligence. Elsevier, 1973. ISBN 9780123852502. Disponível em: <https://linkinghub.elsevier.com/retrieve/pii/ B9780123852502X50019>. Cited on page 70.

KISELEV, V. G.; HAHN, K. R.; AUER, D. P. Is the brain cortex a fractal? NeuroImage, v. 20, n. 3, p. 1765-1774, nov. 2003. ISSN 1053-8119. Disponível em: $<$ https://www.sciencedirect.com/science/article/pii/S105381190300380X > . Cited on page 70 .

KWAN, R. K. S.; EVANS, A. C.; PIKE, G. B. An extensible MRI simulator for post-processing evaluation. In: HöHNE, K. H.; KIKINIS, R. (Ed.). Visualization in Biomedical Computing. Berlin, Heidelberg: Springer, 1996. (Lecture Notes in Computer Science), p. 135-140. ISBN 9783540707394. Cited on page 73.

KWAN, R.-S.; EVANS, A.; PIKE, G. MRI simulation-based evaluation of image-processing and classification methods. IEEE Transactions on Medical Imaging, v. 18, n. 11, p. 1085-1097, nov. 1999. ISSN 1558-254X. Cited on page 73.

LAUTERBUR, P. C. Image Formation by Induced Local Interactions: Examples Employing Nuclear Magnetic Resonance. Nature, Nature Publishing Group, v. 242, n. 5394, p. 190-191, mar. 1973. ISSN 1476-4687. Disponível em: <https://www.nature.com/articles/242190a0>. Cited on page 28.

LOECKX, D. et al. Nonrigid Image Registration Using Conditional Mutual Information. IEEE Transactions on Medical Imaging, v. 29, n. 1, p. 19-29, jan. 2010. ISSN 1558-254X. Cited on page 57.

LYRA, M. L.; TSALLIS, C. Nonextensivity and Multifractality in Low-Dimensional Dissipative Systems. Physical Review Letters, American Physical Society, v. 80, n. 1, p. 53-56, jan. 1998. Disponível em: < https://link.aps.org/doi/10.1103/PhysRevLett.80.53>. Cited on page 66.

MAES, F. et al. Multimodality image registration by maximization of mutual information. IEEE Transactions on Medical Imaging, v. 16, n. 2, p. 187-198, April 1997. ISSN 0278-0062. Cited on page 57.

MAGUIRE, G. Q. et al. Correlation Methods for Tomographic Images Using Two and Three Dimensional Techniques. In: BACHARACH, S. L. (Ed.). Dordrecht: Springer Netherlands, 1986. p. 266-279. ISBN 9789400942615. Disponível em: <https://doi.org/10.1007/978-94-009-4261-5_20>. Cited on page 43.

MAJUMDAR, S.; PRASAD, R. R. The fractal dimension of cerebral surfaces using magnetic resonance images. Computers in Physics, American Institute of Physics, v. 2, n. 6, p. 69-73, nov. 1988. ISSN 0894-1866. Disponível em: $<$ https://aip.scitation.org/doi/abs/10.1063/1.168314>. Cited on page 70. 
MANDELBROT, B. How Long Is the Coast of Britain? Statistical Self-Similarity and Fractional Dimension. Science, American Association for the Advancement of Science, v. 156, n. 3775, p. 636-638, maio 1967. ISSN 0036-8075, 1095-9203. Disponível em: $<$ https://science.sciencemag.org/content/156/3775/636>. Cited on page 59.

MANSFIELD, P.; GRANNELL, P. K. "Diffraction" and microscopy in solids and liquids by NMR. Physical Review B, American Physical Society, v. 12, n. 9, p. 3618-3634, nov. 1975. Disponível em: <https://link.aps.org/doi/10.1103/PhysRevB.12.3618>. Cited on page 28 .

MARZI, C. et al. Toward a more reliable characterization of fractal properties of the cerebral cortex of healthy subjects during the lifespan. Scientific Reports, Nature Publishing Group, v. 10, n. 1, p. 16957, out. 2020. ISSN 2045-2322. Disponível em: <https://www.nature.com/articles/s41598-020-73961-w>. Cited 2 times on pages 71 and 72 .

MEDICAL imaging — Wikipedia, The Free Encyclopedia. 2017. Disponível em: $<$ https://en.wikipedia.org/w/index.php?title=Medical_imaging\&oldid $=779457360>$. Acesso em: 27 de maio de 2017. Cited on page 30.

NICéPHORE Niépce. 2021. Page Version ID: 1009043532. Disponível em: $<$ https://en.wikipedia.org/w/index.php?title=Nic\%C3\%A9phore_Ni\%C3\%A9pce\& oldid $=1009043532>$. Cited on page 28 .

OVERVIEW | ParaView. Disponível em: < https://www.paraview.org/overview/>. Cited on page 76 .

PAPOULIS, A. Probability, Random Variables, and Stochastic Processes. McGraw-Hill, 1991. (Communications and signal processing). ISBN 9780070484771. Disponível em: $<$ https://books.google.com.br/books?id=4IwQAQAAIAAJ $>$. Cited 2 times on pages 38 and 39 .

PELIZZARI, C. A. et al. Accurate Three-Dimensional Registration of CT, PET, and/or MR Images of the Brain. Journal of Computer Assisted Tomography, v. 13, n. 1, p. 20-26, fev. 1989. ISSN 0363-8715. Disponível em: <https://journals.lww.com/jcat/Abstract/ 1989/01000/Accurate_Three_Dimensional_Registration_of_CT,.4.aspx >. Cited 2 times on pages 43 and 44 .

PLUIM, J.; MAINTZ, J.; VIERGEVER, M. Image registration by maximization of combined mutual information and gradient information. IEEE Transactions on Medical Imaging, v. 19, n. 8, p. 809-814, ago. 2000. ISSN 1558-254X. Cited on page 57.

PRADHAN, S.; PATRA, D. Enhanced mutual information based medical image registration. IET Image Processing, IET Digital Library, v. 10, n. 5, p. 418-427, maio 2016. ISSN 1751-9667. Disponível em: <https://digital-library.theiet.org/content/journals/10. 1049/iet-ipr.2015.0346>. Cited on page 58.

QUATERNIONS and spatial rotation. 2021. Page Version ID: 1012996432. Disponível em: <https://en.wikipedia.org/w/index.php?title=Quaternions_and_spatial_rotation\& oldid=1012996432>. Cited on page 49. 
RUECKERT, D. et al. Non-rigid registration using higher-order mutual information. In: Medical Imaging 2000: Image Processing. International Society for Optics and Photonics, 2000. v. 3979, p. 438-447. Disponível em: $<$ https://www.spiedigitallibrary.org/ conference-proceedings-of-spie/3979/0000/ Non-rigid-registration-using-higher-order-mutual-information/10.1117/12.804801. short $>$. Cited on page 57.

RUSSAKOFF, D. B. et al. Image Similarity Using Mutual Information of Regions. In: PAJDLA, T.; MATAS, J. (Ed.). Computer Vision - ECCV 2004. Berlin, Heidelberg: Springer, 2004. (Lecture Notes in Computer Science), p. 596-607. ISBN 9783540246725. Cited on page 57.

RéNYI, A. On Measures of Entropy and Information. Proceedings of the Fourth Berkeley Symposium on Mathematical Statistics and Probability, Volume 1: Contributions to the Theory of Statistics, University of California Press, Berkeley, Calif., p. 547-561, jan. 1961. Disponível em: <https://projecteuclid. org/ebooks / berkeley-symposium-on-mathematical-statistics-and-probability / Proceedings-of-the-Fourth-Berkeley-Symposium-on-Mathematical-Statistics-and/ chapter/On-Measures-of-Entropy-and-Information/bsmsp/1200512181>. Cited on page 66.

SHANNON, C. E.; WEAVER, W. The Mathematical Theory of Communication. University of Illinois Press, 1963. (Illini books, pt. 11). Google-Books-ID: dk0n_eGcqsUC. ISBN 9780252725487. Disponível em: < https://books.google.com.br/books?id=dk0n \ _eGcqsUC $>$. Cited 4 times on pages 34, 35, 36, and 38 .

SPARAVIGNA, A. C. Mutual Information and Nonadditive Entropies: The Case of Tsallis Entropy. Rochester, NY, 2015. Disponível em: <https://papers.ssrn.com/abstract= $3177703>$. Cited on page 65.

STEINHAUS, H. Length, shape and area. Colloquium Mathematicum, Instytut Matematyczny Polskiej Akademii Nauk, v. 3, p. 1-13, 1954. ISSN 0010-1354, 1730-6302. Disponível em: <https://www.impan.pl/en/publishing-house/journals-and-series/ colloquium-mathematicum/all/3/1/93999/length-shape-and-area $>$. Cited on page 60 .

STUDHOLME, C.; HILL, D. L. G.; HAWKES, D. J. An overlap invariant entropy measure of 3D medical image alignment. Pattern Recognition, v. 32, n. 1, p. 71-86, jan. 1999. ISSN 0031-3203. Disponível em: < http://www.sciencedirect.com/science/article/pii/ S0031320398000910>. Cited on page 57 .

TSALLIS, C. Possible generalization of Boltzmann-Gibbs statistics. Journal of Statistical Physics, Kluwer Academic Publishers-Plenum Publishers, v. 52, n. 1-2, p. 479-487, jul 1988. ISSN 0022-4715. Disponível em: <http://link.springer.com/10.1007/BF01016429>. Cited on page 63.

TSALLIS, C. Nonextensive physics: a possible connection between generalized statistical mechanics and quantum groups. Physics Letters A, v. 195, n. 5, p. 329-334, dez. 1994. ISSN 0375-9601. Disponível em: <http://www.sciencedirect.com/science/article/pii/ 037596019490037X $>$. Cited on page 64.

TSALLIS, C. What are the numbers that experiments provide? Quimica Nova, Quimica Nova, v. 17, n. 6, p. 468-471, 1994. ISSN 0100-4042. Disponível em: < http: 
//quimicanova.sbq.org.br/imagebank/pdf/Vol17No6_468_v17_n6_\%283\%29.pdf>. Cited on page 64.

TSALLIS, C. Generalized entropy-based criterion for consistent testing. Physical Review E, American Physical Society, v. 58, n. 2, p. 1442-1445, ago. 1998. Disponível em: $<$ https://link.aps.org/doi/10.1103/PhysRevE.58.1442>. Cited on page 64.

TSALLIS, C. Nonextensive statistics: theoretical, experimental and computational evidences and connections. Brazilian Journal of Physics, Sociedade Brasileira de F\&iacute;sica, v. 29, n. 1, p. 1-35, mar. 1999. ISSN 0103-9733. Disponível em: < http: //www.scielo.br/scielo.php?script=sci_abstract\&pid=S0103-97331999000100002\&lng $=$ en\&nrm $=$ iso\&tlng $=\mathrm{en}>$. Cited 2 times on pages 63 and 64 .

TSALLIS, C. Introduction to Nonextensive Statistical Mechanics: Approaching a Complex World. New York: Springer-Verlag, 2009. ISBN 9780387853581. Disponível em: $<$ https://www.springer.com/us/book/9780387853581>. Cited on page 68.

Van Essen, D. C. et al. Informatics and Data Mining Tools and Strategies for the Human Connectome Project. Frontiers in Neuroinformatics, v. 5, n. June, p. 1-12, 2011. ISSN 1662-5196. Cited on page 75.

VIOLA, P.; WELLS, W. M. Alignment by Maximization of Mutual Information. Int. J. Comput. Vision, Springer Science and Business Media LLC, v. 24, n. 2, p. 137-154, 1997. ISSN 1573-1405. Disponível em: <https://doi.org/10.1023\%2Fa\%3A1007958904918>. Cited 3 times on pages 19, 42, and 57 .

WAHNER, H. W. et al. Comparison of Dual-Energy X-Ray Absorptiometry and Dual Photon Absorptiometry for Bone Mineral Measurements of the Lumbar Spine. Mayo Clinic Proceedings, v. 63, n. 11, p. 1075-1084, nov. 1988. ISSN 0025-6196. Disponível em: $<$ https://www.sciencedirect.com/science/article/pii/S0025619612655025>. Cited on page 28 .

WELLS, W. M. et al. Multi-modal volume registration by maximization of mutual information. Medical Image Analysis, v. 1, n. 1, p. 35-51, mar. 1996. ISSN 1361-8415. Disponível em: <http://www.sciencedirect.com/science/article/pii/S1361841501800049>. Cited on page 57.

WOODS, R. P.; CHERRY, S. R.; MAZZIOTTA, J. C. Rapid Automated Algorithm for Aligning and Reslicing PET Images. Journal of Computer Assisted Tomography, v. 16, n. 4, p. 620-633, jul. 1992. ISSN 0363-8715. Disponível em: < https://journals.lww.com/jcat/ Abstract/1992/07000/Rapid_Automated_Algorithm_for_Aligning_and.24.aspx > . Cited on page 44.

YAMANO, T. Information theory based on nonadditive information content. Phys. Rev. E, American Physical Society, v. 63, p. 046105, Mar 2001. Disponível em: $<$ https://link.aps.org/doi/10.1103/PhysRevE.63.046105>. Cited on page 65.

ZHUANG, X. et al. A Nonrigid Registration Framework Using Spatially Encoded Mutual Information and Free-Form Deformations. IEEE Transactions on Medical Imaging, v. 30, n. 10, p. 1819-1828, out. 2011. ISSN 1558-254X. Cited on page 58. 
ZONNEVELD, F. W. Spectacular rediscovery of the original prints of radiographs Roentgen sent to Lorentz in 1896. Insights into Imaging, v. 11, n. 1, p. 46, mar. 2020. ISSN 1869-4101. Disponível em: <https://doi.org/10.1186/s13244-020-00846-x>. Cited on page 29 . 



\section{APPENDIX A - Full data collected}

A.1 Full data collected from 3D images and 3D transformations

This section contains all the information collected on sec. 4.3, for multiple settings of histogram binning. 


\begin{tabular}{|c|c|c|c|c|c|c|c|c|c|c|c|c|c|c|c|c|c|c|c|c|}
\hline \multirow{3}{*}{$q$} & \multicolumn{5}{|c|}{ Rotation } & \multicolumn{5}{|c|}{ Scaling } & \multicolumn{5}{|c|}{ Skewness } & \multicolumn{5}{|c|}{ Translation } \\
\hline & \multirow{2}{*}{ Mattes } & \multicolumn{2}{|c|}{ Renyi } & \multicolumn{2}{|c|}{ Tsallis } & \multirow{2}{*}{ Mattes } & \multicolumn{2}{|c|}{ Renyi } & \multicolumn{2}{|c|}{ Tsallis } & \multirow{2}{*}{ Mattes } & \multicolumn{2}{|c|}{ Renyi } & \multicolumn{2}{|c|}{ Tsallis } & \multirow{2}{*}{ Mattes } & \multicolumn{2}{|c|}{ Renyi } & \multicolumn{2}{|c|}{ Tsallis } \\
\hline & & A. & N. & A. & $\mathrm{N}$. & & A. & $\mathrm{N}$. & A. & $\mathrm{N}$. & & A. & N. & A. & N. & & A. & $\mathrm{N}$. & A. & $\mathrm{N}$. \\
\hline 0 & 0.863 & 0.101 & 0.122 & 0.089 & 0.125 & 0.988 & 0.164 & 0.273 & 0.12 & 0.223 & 1.0 & 0.042 & 0.255 & 0.039 & 0.202 & 0.658 & 0.002 & 0.259 & 0.001 & 0.191 \\
\hline 0.1 & 0.863 & 0.236 & 0.455 & 0.115 & 0.455 & 0.988 & 0.201 & 0.785 & 0.155 & 0.376 & 1.0 & 0.056 & 0.813 & 0.043 & 0.321 & 0.658 & 0.002 & 0.53 & 0.001 & 0.299 \\
\hline 0.2 & 0.863 & 0.41 & 0.599 & 0.143 & 0.593 & 0.988 & 0.2 & 0.802 & 0.202 & 0.538 & 1.0 & 0.101 & 0.963 & 0.049 & 0.42 & 0.658 & 0.003 & 0.944 & 0.001 & 0.302 \\
\hline 0.3 & 0.863 & 0.523 & 0.658 & 0.195 & 0.623 & 0.988 & 0.271 & 0.75 & 0.255 & 0.669 & 1.0 & 0.194 & 0.999 & 0.058 & 0.658 & 0.658 & 0.008 & 0.992 & 0.001 & 0.271 \\
\hline 0.4 & 0.863 & 0.729 & 0.612 & 0.258 & 0.728 & 0.988 & 0.343 & 0.753 & 0.283 & 0.697 & 1.0 & 0.338 & 1.0 & 0.064 & 0.819 & 0.658 & 0.011 & 0.999 & 0.001 & 0.133 \\
\hline 0.5 & 0.863 & 0.752 & 0.588 & 0.283 & 0.74 & 0.988 & 0.473 & 0.748 & 0.304 & 0.701 & 1.0 & 0.573 & 1.0 & 0.069 & 0.894 & 0.658 & 0.022 & 1.0 & 0.002 & 0.067 \\
\hline 0.6 & 0.863 & 0.721 & 0.606 & 0.301 & 0.706 & 0.988 & 0.632 & 0.746 & 0.332 & 0.715 & 1.0 & 0.832 & 1.0 & 0.076 & 0.961 & 0.658 & 0.037 & 1.0 & 0.003 & 0.079 \\
\hline 0.7 & 0.863 & 0.695 & 0.654 & 0.391 & 0.703 & 0.988 & 0.737 & 0.747 & 0.336 & 0.749 & 1.0 & 0.971 & 1.0 & 0.083 & 0.993 & 0.658 & 0.068 & 1.0 & 0.003 & 0.13 \\
\hline 0.8 & 0.863 & 0.753 & 0.672 & 0.63 & 0.754 & 0.988 & 0.828 & 0.751 & 0.35 & 0.826 & 1.0 & 1.0 & 1.0 & 0.092 & 1.0 & 0.658 & 0.119 & 1.0 & 0.004 & 0.237 \\
\hline 0.9 & 0.863 & 0.837 & 0.725 & 0.802 & 0.831 & 0.988 & 0.978 & 0.801 & 0.392 & 0.996 & 1.0 & 1.0 & 1.0 & 0.147 & 1.0 & 0.658 & 0.552 & 1.0 & 0.008 & 0.93 \\
\hline 0.91 & 0.863 & 0.836 & 0.742 & 0.807 & 0.833 & 0.988 & 0.992 & 0.81 & 0.411 & 0.996 & 1.0 & 1.0 & 1.0 & 0.16 & 1.0 & 0.658 & 0.656 & 1.0 & 0.01 & 0.961 \\
\hline 0.92 & 0.863 & 0.845 & 0.752 & 0.806 & 0.842 & 0.988 & 0.994 & 0.825 & 0.422 & 0.995 & 1.0 & 1.0 & 1.0 & 0.181 & 1.0 & 0.658 & 0.766 & 1.0 & 0.011 & 0.969 \\
\hline 0.93 & 0.863 & 0.842 & 0.749 & 0.806 & 0.838 & 0.988 & 0.995 & 0.845 & 0.443 & 0.995 & 1.0 & 1.0 & 1.0 & 0.205 & 1.0 & 0.658 & 0.922 & 1.0 & 0.013 & 0.98 \\
\hline 0.94 & 0.863 & 0.846 & 0.77 & 0.804 & 0.843 & 0.988 & 0.994 & 0.876 & 0.482 & 0.995 & 1.0 & 1.0 & 1.0 & 0.245 & 1.0 & 0.658 & 0.968 & 1.0 & 0.016 & 0.981 \\
\hline 0.95 & 0.863 & 0.838 & 0.786 & 0.814 & 0.839 & 0.988 & 0.995 & 0.964 & 0.546 & 0.995 & 1.0 & 1.0 & 1.0 & 0.312 & 1.0 & 0.658 & 0.977 & 1.0 & 0.02 & 0.984 \\
\hline 0.96 & 0.863 & 0.836 & 0.794 & 0.809 & 0.834 & 0.988 & 0.994 & 0.999 & 0.588 & 0.995 & 1.0 & 1.0 & 1.0 & 0.415 & 1.0 & 0.658 & 0.984 & 1.0 & 0.026 & 0.99 \\
\hline 0.97 & 0.863 & 0.833 & 0.792 & 0.814 & 0.834 & 0.988 & 0.994 & 1.0 & 0.653 & 0.995 & 1.0 & 1.0 & 1.0 & 0.597 & 1.0 & 0.658 & 0.992 & 1.0 & 0.045 & 0.995 \\
\hline 0.98 & 0.863 & 0.834 & 0.804 & 0.815 & 0.831 & 0.988 & 0.994 & 1.0 & 0.799 & 0.995 & 1.0 & 1.0 & 1.0 & 0.812 & 1.0 & 0.658 & 0.998 & 1.0 & 0.086 & 0.998 \\
\hline 0.99 & 0.863 & 0.826 & 0.809 & 0.815 & 0.826 & 0.988 & 0.994 & 0.998 & 0.992 & 0.994 & 1.0 & 1.0 & 1.0 & 0.977 & 1.0 & 0.658 & 1.0 & 1.0 & 0.564 & 1.0 \\
\hline 0.9999 & 0.863 & 0.813 & 0.816 & 0.814 & 0.813 & 0.988 & 0.994 & 0.994 & 0.994 & 0.994 & 1.0 & 1.0 & 1.0 & 1.0 & 1.0 & 0.658 & 1.0 & 1.0 & 1.0 & 1.0 \\
\hline 1 & 0.863 & 0.814 & 0.814 & 0.814 & 0.814 & 0.988 & 0.994 & 0.994 & 0.994 & 0.994 & 1.0 & 1.0 & 1.0 & 1.0 & 1.0 & 0.658 & 1.0 & 1.0 & 1.0 & 1.0 \\
\hline
\end{tabular}

Table 6 - Metrics registration percentage as function of the entropic index $q$, limited in the range $q \leq 1$, with 12 bits histogram binning. (A. means additive, N. means non-additive) 


\begin{tabular}{|c|c|c|c|c|c|c|c|c|c|c|c|c|c|c|c|c|c|c|c|c|}
\hline \multirow{3}{*}{$q$} & \multicolumn{5}{|c|}{ Rotation } & \multicolumn{5}{|c|}{ Scaling } & \multicolumn{5}{|c|}{ Skewness } & \multicolumn{5}{|c|}{ Translation } \\
\hline & \multirow{2}{*}{ Mattes } & \multicolumn{2}{|c|}{ Renyi } & \multicolumn{2}{|c|}{ Tsallis } & \multirow{2}{*}{ Mattes } & \multicolumn{2}{|c|}{ Renyi } & \multicolumn{2}{|c|}{ Tsallis } & \multirow{2}{*}{ Mattes } & \multicolumn{2}{|c|}{ Renyi } & \multicolumn{2}{|c|}{ Tsallis } & \multirow{2}{*}{ Mattes } & \multicolumn{2}{|c|}{ Renyi } & \multicolumn{2}{|c|}{ Tsallis } \\
\hline & & A. & $\mathrm{N}$. & A. & N. & & A. & N. & A. & $\mathrm{N}$. & & A. & $\mathrm{N}$. & A. & $\mathrm{N}$. & & A. & $\mathrm{N}$. & A. & N. \\
\hline 1 & 0.863 & 0.814 & 0.814 & 0.814 & 0.814 & 0.988 & 0.994 & 0.994 & 0.994 & 0.994 & 1.0 & 1.0 & 1.0 & 1.0 & 1.0 & 0.658 & 1.0 & 1.0 & 1.0 & 1.0 \\
\hline 1.0001 & 0.863 & 0.814 & 0.813 & 0.816 & 0.814 & 0.988 & 0.994 & 0.994 & 0.994 & 0.994 & 1.0 & 1.0 & 1.0 & 1.0 & 1.0 & 0.658 & 1.0 & 1.0 & 1.0 & 1.0 \\
\hline 1.01 & 0.863 & 0.806 & 0.784 & 0.801 & 0.806 & 0.988 & 0.994 & 0.992 & 0.997 & 0.994 & 1.0 & 1.0 & 0.983 & 1.0 & 1.0 & 0.658 & 0.999 & 0.742 & 1.0 & 0.999 \\
\hline 1.02 & 0.863 & 0.798 & 0.768 & 0.789 & 0.792 & 0.988 & 0.994 & 0.784 & 1.0 & 0.994 & 1.0 & 1.0 & 0.82 & 1.0 & 1.0 & 0.658 & 0.996 & 0.144 & 1.0 & 0.996 \\
\hline 1.03 & 0.863 & 0.783 & 0.764 & 0.773 & 0.778 & 0.988 & 0.994 & 0.597 & 1.0 & 0.993 & 1.0 & 1.0 & 0.627 & 1.0 & 1.0 & 0.658 & 0.989 & 0.055 & 1.0 & 0.989 \\
\hline 1.04 & 0.863 & 0.767 & 0.767 & 0.76 & 0.766 & 0.988 & 0.994 & 0.508 & 1.0 & 0.993 & 1.0 & 1.0 & 0.403 & 1.0 & 1.0 & 0.658 & 0.987 & 0.032 & 1.0 & 0.987 \\
\hline 1.05 & 0.863 & 0.756 & 0.757 & 0.748 & 0.748 & 0.988 & 0.994 & 0.398 & 1.0 & 0.993 & 1.0 & 1.0 & 0.246 & 1.0 & 1.0 & 0.658 & 0.984 & 0.021 & 1.0 & 0.984 \\
\hline 1.06 & 0.863 & 0.741 & 0.69 & 0.735 & 0.735 & 0.988 & 0.994 & 0.352 & 1.0 & 0.993 & 1.0 & 1.0 & 0.166 & 1.0 & 1.0 & 0.658 & 0.983 & 0.014 & 1.0 & 0.983 \\
\hline 1.07 & 0.863 & 0.73 & 0.343 & 0.736 & 0.727 & 0.988 & 0.994 & 0.295 & 0.998 & 0.993 & 1.0 & 1.0 & 0.117 & 1.0 & 1.0 & 0.658 & 0.983 & 0.009 & 1.0 & 0.984 \\
\hline 1.08 & 0.863 & 0.718 & 0.073 & 0.717 & 0.723 & 0.988 & 0.993 & 0.277 & 0.971 & 0.993 & 1.0 & 1.0 & 0.086 & 1.0 & 1.0 & 0.658 & 0.983 & 0.007 & 1.0 & 0.984 \\
\hline 1.09 & 0.863 & 0.708 & 0.001 & 0.721 & 0.718 & 0.988 & 0.993 & 0.237 & 0.907 & 0.993 & 1.0 & 1.0 & 0.064 & 1.0 & 1.0 & 0.658 & 0.983 & 0.006 & 1.0 & 0.984 \\
\hline 1.1 & 0.863 & 0.702 & 0.0 & 0.706 & 0.713 & 0.988 & 0.993 & 0.23 & 0.824 & 0.993 & 1.0 & 1.0 & 0.049 & 1.0 & 1.0 & 0.658 & 0.982 & 0.004 & 1.0 & 0.983 \\
\hline 1.2 & 0.863 & 0.629 & 0.0 & 0.643 & 0.61 & 0.988 & 0.993 & 0.178 & 0.717 & 0.626 & 1.0 & 1.0 & 0.006 & 1.0 & 0.832 & 0.658 & 0.98 & 0.001 & 1.0 & 0.966 \\
\hline 1.3 & 0.863 & 0.575 & 0.0 & 0.583 & 0.0 & 0.988 & 0.993 & 0.173 & 0.706 & 0.232 & 1.0 & 1.0 & 0.001 & 1.0 & 0.271 & 0.658 & 0.981 & 0.001 & 1.0 & 0.881 \\
\hline 1.4 & 0.863 & 0.537 & 0.0 & 0.56 & 0.0 & 0.988 & 0.993 & 0.172 & 0.702 & 0.183 & 1.0 & 1.0 & 0.0 & 1.0 & 0.008 & 0.658 & 0.982 & 0.001 & 1.0 & 0.775 \\
\hline 1.5 & 0.863 & 0.522 & 0.0 & 0.522 & 0.0 & 0.988 & 0.994 & 0.172 & 0.702 & 0.179 & 1.0 & 0.999 & 0.0 & 1.0 & 0.003 & 0.658 & 0.982 & 0.001 & 1.0 & 0.626 \\
\hline 1.6 & 0.863 & 0.513 & 0.0 & 0.502 & 0.0 & 0.988 & 0.994 & 0.172 & 0.701 & 0.172 & 1.0 & 0.999 & 0.0 & 1.0 & 0.002 & 0.658 & 0.982 & 0.001 & 1.0 & 0.306 \\
\hline 1.7 & 0.863 & 0.513 & 0.0 & 0.501 & 0.0 & 0.988 & 0.994 & 0.156 & 0.702 & 0.171 & 1.0 & 1.0 & 0.0 & 1.0 & 0.001 & 0.658 & 0.982 & 0.001 & 1.0 & 0.246 \\
\hline 1.8 & 0.863 & 0.514 & 0.0 & 0.493 & 0.0 & 0.988 & 0.994 & 0.156 & 0.704 & 0.174 & 1.0 & 1.0 & 0.0 & 1.0 & 0.001 & 0.658 & 0.983 & 0.0 & 1.0 & 0.162 \\
\hline 1.9 & 0.863 & 0.512 & 0.0 & 0.494 & 0.0 & 0.988 & 0.994 & 0.156 & 0.705 & 0.174 & 1.0 & 1.0 & 0.0 & 1.0 & 0.001 & 0.658 & 0.982 & 0.0 & 1.0 & 0.102 \\
\hline 2 & 0.863 & 0.507 & 0.0 & 0.496 & 0.0 & 0.988 & 0.987 & 0.14 & 0.707 & 0.174 & 1.0 & 1.0 & 0.0 & 1.0 & 0.001 & 0.658 & 0.982 & 0.0 & 1.0 & 0.101 \\
\hline 3 & 0.863 & 0.483 & 0.0 & 0.483 & 0.0 & 0.988 & 0.954 & 0.124 & 0.725 & 0.179 & 1.0 & 1.0 & 0.0 & 1.0 & 0.001 & 0.658 & 0.982 & 0.0 & 1.0 & 0.001 \\
\hline
\end{tabular}

Table 7 - Metrics registration percentage as function of the entropic index $q$, limited in the range $q \geq 1$, with 12 bits histogram binning. (A. means additive, N. means non-additive) 


\begin{tabular}{|c|c|c|c|c|c|c|c|c|c|c|c|c|c|c|c|c|c|c|c|c|}
\hline \multirow{3}{*}{$q$} & \multicolumn{5}{|c|}{ Rotation } & \multicolumn{5}{|c|}{ Scaling } & \multicolumn{5}{|c|}{ Skewness } & \multicolumn{5}{|c|}{ Translation } \\
\hline & \multirow{2}{*}{ Mattes } & \multicolumn{2}{|c|}{ Renyi } & \multicolumn{2}{|c|}{ Tsallis } & \multirow{2}{*}{ Mattes } & \multicolumn{2}{|c|}{ Renyi } & \multicolumn{2}{|c|}{ Tsallis } & \multirow{2}{*}{ Mattes } & \multicolumn{2}{|c|}{ Renyi } & \multicolumn{2}{|c|}{ Tsallis } & \multirow{2}{*}{ Mattes } & \multicolumn{2}{|c|}{ Renyi } & \multicolumn{2}{|c|}{ Tsallis } \\
\hline & & A. & N. & A. & N. & & A. & $\mathrm{N}$. & A. & N. & & A. & N. & A. & N. & & A. & N. & A. & N. \\
\hline 0 & 0.863 & 0.007 & 0.017 & 0.007 & 0.009 & 0.988 & 0.012 & 0.184 & 0.006 & 0.01 & 1.0 & 0.006 & 0.012 & 0.003 & 0.008 & 0.658 & 0.001 & 0.215 & 0.002 & 0.001 \\
\hline 0.1 & 0.863 & 0.009 & 0.026 & 0.008 & 0.01 & 0.988 & 0.013 & 0.684 & 0.027 & 0.026 & 1.0 & 0.008 & 0.331 & 0.006 & 0.01 & 0.658 & 0.001 & 0.54 & 0.002 & 0.001 \\
\hline 0.2 & 0.863 & 0.018 & 0.092 & 0.011 & 0.019 & 0.988 & 0.052 & 0.736 & 0.041 & 0.15 & 1.0 & 0.016 & 0.872 & 0.014 & 0.028 & 0.658 & 0.002 & 0.893 & 0.001 & 0.044 \\
\hline 0.3 & 0.863 & 0.025 & 0.321 & 0.016 & 0.025 & 0.988 & 0.16 & 0.72 & 0.074 & 0.228 & 1.0 & 0.11 & 0.973 & 0.029 & 0.138 & 0.658 & 0.003 & 0.982 & 0.001 & 0.046 \\
\hline 0.4 & 0.863 & 0.097 & 0.556 & 0.027 & 0.098 & 0.988 & 0.315 & 0.726 & 0.142 & 0.341 & 1.0 & 0.365 & 0.999 & 0.048 & 0.399 & 0.658 & 0.035 & 0.998 & 0.002 & 0.039 \\
\hline 0.5 & 0.863 & 0.32 & 0.598 & 0.056 & 0.31 & 0.988 & 0.526 & 0.733 & 0.223 & 0.523 & 1.0 & 0.706 & 1.0 & 0.061 & 0.682 & 0.658 & 0.048 & 1.0 & 0.003 & 0.042 \\
\hline 0.6 & 0.863 & 0.638 & 0.61 & 0.104 & 0.661 & 0.988 & 0.636 & 0.737 & 0.284 & 0.616 & 1.0 & 0.932 & 1.0 & 0.074 & 0.891 & 0.658 & 0.059 & 1.0 & 0.003 & 0.045 \\
\hline 0.7 & 0.863 & 0.755 & 0.638 & 0.376 & 0.761 & 0.988 & 0.709 & 0.744 & 0.342 & 0.677 & 1.0 & 0.993 & 1.0 & 0.092 & 0.986 & 0.658 & 0.09 & 1.0 & 0.005 & 0.069 \\
\hline 0.8 & 0.863 & 0.764 & 0.674 & 0.731 & 0.768 & 0.988 & 0.791 & 0.752 & 0.403 & 0.776 & 1.0 & 1.0 & 1.0 & 0.124 & 1.0 & 0.658 & 0.142 & 1.0 & 0.006 & 0.13 \\
\hline 0.9 & 0.863 & 0.797 & 0.736 & 0.805 & 0.794 & 0.988 & 0.982 & 0.806 & 0.521 & 0.985 & 1.0 & 1.0 & 1.0 & 0.239 & 1.0 & 0.658 & 0.405 & 1.0 & 0.015 & 0.526 \\
\hline 0.91 & 0.863 & 0.797 & 0.746 & 0.805 & 0.797 & 0.988 & 0.988 & 0.816 & 0.533 & 0.989 & 1.0 & 1.0 & 1.0 & 0.266 & 1.0 & 0.658 & 0.512 & 1.0 & 0.017 & 0.674 \\
\hline 0.92 & 0.863 & 0.799 & 0.752 & 0.803 & 0.798 & 0.988 & 0.99 & 0.834 & 0.568 & 0.992 & 1.0 & 1.0 & 1.0 & 0.309 & 1.0 & 0.658 & 0.636 & 1.0 & 0.02 & 0.822 \\
\hline 0.93 & 0.863 & 0.812 & 0.756 & 0.803 & 0.803 & 0.988 & 0.991 & 0.864 & 0.59 & 0.992 & 1.0 & 1.0 & 1.0 & 0.363 & 1.0 & 0.658 & 0.736 & 1.0 & 0.025 & 0.886 \\
\hline 0.94 & 0.863 & 0.813 & 0.754 & 0.799 & 0.809 & 0.988 & 0.992 & 0.919 & 0.616 & 0.992 & 1.0 & 1.0 & 1.0 & 0.429 & 1.0 & 0.658 & 0.847 & 1.0 & 0.03 & 0.949 \\
\hline 0.95 & 0.863 & 0.809 & 0.762 & 0.8 & 0.811 & 0.988 & 0.992 & 0.993 & 0.657 & 0.992 & 1.0 & 1.0 & 1.0 & 0.527 & 1.0 & 0.658 & 0.93 & 1.0 & 0.042 & 0.952 \\
\hline 0.96 & 0.863 & 0.806 & 0.776 & 0.796 & 0.81 & 0.988 & 0.992 & 0.999 & 0.711 & 0.992 & 1.0 & 1.0 & 1.0 & 0.653 & 1.0 & 0.658 & 0.937 & 1.0 & 0.055 & 0.959 \\
\hline 0.97 & 0.863 & 0.803 & 0.783 & 0.798 & 0.804 & 0.988 & 0.992 & 0.998 & 0.815 & 0.992 & 1.0 & 1.0 & 1.0 & 0.787 & 1.0 & 0.658 & 0.939 & 1.0 & 0.089 & 0.965 \\
\hline 0.98 & 0.863 & 0.8 & 0.796 & 0.796 & 0.797 & 0.988 & 0.992 & 0.996 & 0.922 & 0.993 & 1.0 & 1.0 & 1.0 & 0.916 & 1.0 & 0.658 & 0.962 & 1.0 & 0.202 & 0.964 \\
\hline 0.99 & 0.863 & 0.8 & 0.797 & 0.802 & 0.797 & 0.988 & 0.993 & 0.994 & 0.991 & 0.993 & 1.0 & 1.0 & 1.0 & 0.995 & 1.0 & 0.658 & 0.975 & 1.0 & 0.621 & 0.977 \\
\hline 0.9999 & 0.863 & 0.796 & 0.796 & 0.796 & 0.796 & 0.988 & 0.993 & 0.993 & 0.993 & 0.993 & 1.0 & 1.0 & 1.0 & 1.0 & 1.0 & 0.658 & 0.982 & 0.984 & 0.98 & 0.982 \\
\hline 1 & 0.863 & 0.796 & 0.796 & 0.796 & 0.796 & 0.988 & 0.993 & 0.993 & 0.993 & 0.993 & 1.0 & 1.0 & 1.0 & 1.0 & 1.0 & 0.658 & 0.982 & 0.982 & 0.982 & 0.982 \\
\hline
\end{tabular}

Table 8 - Metrics registration percentage as function of the entropic index $q$, limited in the range $q \leq 1$, with 10 bits histogram binning. (A. means additive, N. means non-additive) 


\begin{tabular}{|c|c|c|c|c|c|c|c|c|c|c|c|c|c|c|c|c|c|c|c|c|}
\hline \multirow{3}{*}{$q$} & \multicolumn{5}{|c|}{ Rotation } & \multicolumn{5}{|c|}{ Scaling } & \multicolumn{5}{|c|}{ Skewness } & \multicolumn{5}{|c|}{ Translation } \\
\hline & \multirow{2}{*}{ Mattes } & \multicolumn{2}{|c|}{ Renyi } & \multicolumn{2}{|c|}{ Tsallis } & \multirow{2}{*}{ Mattes } & \multicolumn{2}{|c|}{ Renyi } & \multicolumn{2}{|c|}{ Tsallis } & \multirow{2}{*}{ Mattes } & \multicolumn{2}{|c|}{ Renyi } & \multicolumn{2}{|c|}{ Tsallis } & \multirow{2}{*}{ Mattes } & \multicolumn{2}{|c|}{ Renyi } & \multicolumn{2}{|c|}{ Tsallis } \\
\hline & & A. & N. & A. & N. & & A. & $\mathrm{N}$. & A. & N. & & A. & N. & A. & N. & & A. & $\mathrm{N}$. & A. & $\mathrm{N}$. \\
\hline 1 & 0.863 & 0.796 & 0.796 & 0.796 & 0.796 & 0.988 & 0.993 & 0.993 & 0.993 & 0.993 & 1.0 & 1.0 & 1.0 & 1.0 & 1.0 & 0.658 & 0.982 & 0.982 & 0.982 & 0.982 \\
\hline 1.0001 & 0.863 & 0.796 & 0.796 & 0.796 & 0.796 & 0.988 & 0.993 & 0.993 & 0.993 & 0.993 & 1.0 & 1.0 & 1.0 & 1.0 & 1.0 & 0.658 & 0.982 & 0.98 & 0.984 & 0.982 \\
\hline 1.01 & 0.863 & 0.793 & 0.789 & 0.794 & 0.792 & 0.988 & 0.993 & 0.992 & 0.994 & 0.993 & 1.0 & 1.0 & 0.997 & 1.0 & 1.0 & 0.658 & 0.988 & 0.748 & 1.0 & 0.988 \\
\hline 1.02 & 0.863 & 0.792 & 0.777 & 0.787 & 0.79 & 0.988 & 0.993 & 0.949 & 0.996 & 0.993 & 1.0 & 1.0 & 0.936 & 1.0 & 1.0 & 0.658 & 0.984 & 0.447 & 1.0 & 0.984 \\
\hline 1.03 & 0.863 & 0.782 & 0.768 & 0.771 & 0.783 & 0.988 & 0.993 & 0.814 & 0.998 & 0.993 & 1.0 & 1.0 & 0.834 & 1.0 & 1.0 & 0.658 & 0.978 & 0.164 & 1.0 & 0.978 \\
\hline 1.04 & 0.863 & 0.775 & 0.769 & 0.763 & 0.775 & 0.988 & 0.993 & 0.686 & 1.0 & 0.992 & 1.0 & 1.0 & 0.725 & 1.0 & 1.0 & 0.658 & 0.972 & 0.102 & 1.0 & 0.972 \\
\hline 1.05 & 0.863 & 0.771 & 0.768 & 0.754 & 0.767 & 0.988 & 0.992 & 0.6 & 1.0 & 0.992 & 1.0 & 1.0 & 0.611 & 1.0 & 1.0 & 0.658 & 0.972 & 0.066 & 1.0 & 0.972 \\
\hline 1.06 & 0.863 & 0.764 & 0.772 & 0.748 & 0.763 & 0.988 & 0.993 & 0.557 & 1.0 & 0.993 & 1.0 & 1.0 & 0.491 & 1.0 & 1.0 & 0.658 & 0.972 & 0.046 & 1.0 & 0.973 \\
\hline 1.07 & 0.863 & 0.755 & 0.773 & 0.743 & 0.752 & 0.988 & 0.993 & 0.516 & 1.0 & 0.993 & 1.0 & 1.0 & 0.388 & 1.0 & 1.0 & 0.658 & 0.974 & 0.034 & 1.0 & 0.974 \\
\hline 1.08 & 0.863 & 0.754 & 0.768 & 0.732 & 0.745 & 0.988 & 0.993 & 0.451 & 1.0 & 0.993 & 1.0 & 1.0 & 0.307 & 1.0 & 1.0 & 0.658 & 0.974 & 0.026 & 1.0 & 0.975 \\
\hline 1.09 & 0.863 & 0.743 & 0.762 & 0.73 & 0.743 & 0.988 & 0.993 & 0.412 & 1.0 & 0.993 & 1.0 & 1.0 & 0.251 & 1.0 & 1.0 & 0.658 & 0.974 & 0.022 & 1.0 & 0.975 \\
\hline 1.1 & 0.863 & 0.74 & 0.752 & 0.727 & 0.736 & 0.988 & 0.993 & 0.365 & 0.999 & 0.993 & 1.0 & 1.0 & 0.203 & 1.0 & 1.0 & 0.658 & 0.976 & 0.02 & 1.0 & 0.977 \\
\hline 1.2 & 0.863 & 0.698 & 0.001 & 0.701 & 0.71 & 0.988 & 0.993 & 0.233 & 0.738 & 0.981 & 1.0 & 1.0 & 0.052 & 1.0 & 1.0 & 0.658 & 0.976 & 0.005 & 1.0 & 0.978 \\
\hline 1.3 & 0.863 & 0.647 & 0.0 & 0.67 & 0.663 & 0.988 & 0.993 & 0.195 & 0.712 & 0.597 & 1.0 & 1.0 & 0.021 & 1.0 & 0.773 & 0.658 & 0.978 & 0.002 & 1.0 & 0.962 \\
\hline 1.4 & 0.863 & 0.6 & 0.0 & 0.652 & 0.128 & 0.988 & 0.993 & 0.187 & 0.705 & 0.377 & 1.0 & 1.0 & 0.011 & 1.0 & 0.454 & 0.658 & 0.98 & 0.002 & 1.0 & 0.914 \\
\hline 1.5 & 0.863 & 0.569 & 0.0 & 0.645 & 0.0 & 0.988 & 0.994 & 0.172 & 0.696 & 0.233 & 1.0 & 1.0 & 0.007 & 1.0 & 0.212 & 0.658 & 0.981 & 0.001 & 1.0 & 0.847 \\
\hline 1.6 & 0.863 & 0.542 & 0.0 & 0.564 & 0.0 & 0.988 & 0.994 & 0.172 & 0.687 & 0.208 & 1.0 & 1.0 & 0.005 & 1.0 & 0.063 & 0.658 & 0.982 & 0.001 & 1.0 & 0.757 \\
\hline 1.7 & 0.863 & 0.529 & 0.0 & 0.563 & 0.0 & 0.988 & 0.994 & 0.171 & 0.687 & 0.191 & 1.0 & 1.0 & 0.004 & 1.0 & 0.019 & 0.658 & 0.982 & 0.001 & 1.0 & 0.678 \\
\hline 1.8 & 0.863 & 0.524 & 0.0 & 0.56 & 0.0 & 0.988 & 0.994 & 0.171 & 0.694 & 0.183 & 1.0 & 1.0 & 0.003 & 0.999 & 0.013 & 0.658 & 0.982 & 0.001 & 1.0 & 0.601 \\
\hline 1.9 & 0.863 & 0.52 & 0.0 & 0.529 & 0.0 & 0.988 & 0.994 & 0.171 & 0.694 & 0.177 & 1.0 & 0.999 & 0.003 & 0.997 & 0.011 & 0.658 & 0.982 & 0.001 & 1.0 & 0.463 \\
\hline 2 & 0.863 & 0.509 & 0.0 & 0.523 & 0.0 & 0.988 & 0.994 & 0.171 & 0.696 & 0.176 & 1.0 & 0.997 & 0.003 & 0.997 & 0.009 & 0.658 & 0.982 & 0.001 & 1.0 & 0.355 \\
\hline 3 & 0.863 & 0.476 & 0.0 & 0.495 & 0.0 & 0.988 & 0.983 & 0.154 & 0.705 & 0.167 & 1.0 & 0.982 & 0.003 & 0.998 & 0.005 & 0.658 & 0.98 & 0.0 & 1.0 & 0.151 \\
\hline
\end{tabular}

Table 9 - Metrics registration percentage as function of the entropic index $q$, limited in the range $q \geq 1$, with 10 bits histogram binning. (A. means additive, N. means non-additive) 


\begin{tabular}{|c|c|c|c|c|c|c|c|c|c|c|c|c|c|c|c|c|c|c|c|c|}
\hline \multirow{3}{*}{$q$} & \multicolumn{5}{|c|}{ Rotation } & \multicolumn{5}{|c|}{ Scaling } & \multicolumn{5}{|c|}{ Skewness } & \multicolumn{5}{|c|}{ Translation } \\
\hline & \multirow{2}{*}{ Mattes } & \multicolumn{2}{|c|}{ Renyi } & \multicolumn{2}{|c|}{ Tsallis } & \multirow{2}{*}{ Mattes } & \multicolumn{2}{|c|}{ Renyi } & \multicolumn{2}{|c|}{ Tsallis } & \multirow{2}{*}{ Mattes } & \multicolumn{2}{|c|}{ Renyi } & \multicolumn{2}{|c|}{ Tsallis } & \multirow{2}{*}{ Mattes } & \multicolumn{2}{|c|}{ Renyi } & \multicolumn{2}{|c|}{ Tsallis } \\
\hline & & A. & N. & A. & $\mathrm{N}$. & & A. & $\mathrm{N}$. & A. & $\mathrm{N}$. & & A. & $\mathrm{N}$. & A. & $\mathrm{N}$. & & A. & $\mathrm{N}$. & A. & $\mathrm{N}$. \\
\hline 0 & 0.863 & 0.003 & 0.011 & 0.005 & 0.004 & 0.988 & 0.005 & 0.05 & 0.005 & 0.008 & 1.0 & 0.009 & 0.006 & 0.006 & 0.01 & 0.658 & 0.005 & 0.191 & 0.006 & 0.013 \\
\hline 0.1 & 0.863 & 0.011 & 0.016 & 0.012 & 0.009 & 0.988 & 0.014 & 0.488 & 0.018 & 0.011 & 1.0 & 0.011 & 0.304 & 0.007 & 0.012 & 0.658 & 0.009 & 0.481 & 0.004 & 0.02 \\
\hline 0.2 & 0.863 & 0.014 & 0.042 & 0.014 & 0.014 & 0.988 & 0.096 & 0.701 & 0.038 & 0.131 & 1.0 & 0.018 & 0.808 & 0.016 & 0.018 & 0.658 & 0.017 & 0.796 & 0.002 & 0.03 \\
\hline 0.3 & 0.863 & 0.025 & 0.117 & 0.017 & 0.023 & 0.988 & 0.159 & 0.708 & 0.05 & 0.151 & 1.0 & 0.087 & 0.946 & 0.027 & 0.078 & 0.658 & 0.026 & 0.944 & 0.002 & 0.015 \\
\hline 0.4 & 0.863 & 0.046 & 0.327 & 0.023 & 0.042 & 0.988 & 0.275 & 0.717 & 0.1 & 0.238 & 1.0 & 0.353 & 0.992 & 0.053 & 0.29 & 0.658 & 0.028 & 0.987 & 0.002 & 0.007 \\
\hline 0.5 & 0.863 & 0.135 & 0.53 & 0.055 & 0.119 & 0.988 & 0.436 & 0.726 & 0.186 & 0.381 & 1.0 & 0.664 & 0.998 & 0.075 & 0.54 & 0.658 & 0.048 & 0.999 & 0.003 & 0.026 \\
\hline 0.6 & 0.863 & 0.495 & 0.565 & 0.107 & 0.476 & 0.988 & 0.605 & 0.735 & 0.28 & 0.552 & 1.0 & 0.903 & 0.999 & 0.095 & 0.816 & 0.658 & 0.063 & 1.0 & 0.005 & 0.039 \\
\hline 0.7 & 0.863 & 0.736 & 0.578 & 0.486 & 0.751 & 0.988 & 0.692 & 0.745 & 0.367 & 0.671 & 1.0 & 0.986 & 1.0 & 0.122 & 0.97 & 0.658 & 0.087 & 1.0 & 0.007 & 0.066 \\
\hline 0.8 & 0.863 & 0.763 & 0.627 & 0.839 & 0.767 & 0.988 & 0.795 & 0.757 & 0.513 & 0.792 & 1.0 & 0.999 & 1.0 & 0.203 & 0.999 & 0.658 & 0.142 & 1.0 & 0.012 & 0.134 \\
\hline 0.9 & 0.863 & 0.774 & 0.713 & 0.807 & 0.773 & 0.988 & 0.984 & 0.822 & 0.663 & 0.984 & 1.0 & 1.0 & 1.0 & 0.477 & 1.0 & 0.658 & 0.539 & 1.0 & 0.035 & 0.661 \\
\hline 0.91 & 0.863 & 0.783 & 0.727 & 0.804 & 0.782 & 0.988 & 0.987 & 0.851 & 0.685 & 0.987 & 1.0 & 1.0 & 1.0 & 0.544 & 1.0 & 0.658 & 0.637 & 1.0 & 0.037 & 0.687 \\
\hline 0.92 & 0.863 & 0.783 & 0.74 & 0.805 & 0.785 & 0.988 & 0.99 & 0.874 & 0.721 & 0.99 & 1.0 & 1.0 & 1.0 & 0.614 & 1.0 & 0.658 & 0.675 & 1.0 & 0.047 & 0.749 \\
\hline 0.93 & 0.863 & 0.787 & 0.751 & 0.81 & 0.786 & 0.988 & 0.99 & 0.936 & 0.754 & 0.991 & 1.0 & 1.0 & 1.0 & 0.674 & 1.0 & 0.658 & 0.716 & 1.0 & 0.055 & 0.871 \\
\hline 0.94 & 0.863 & 0.792 & 0.762 & 0.812 & 0.79 & 0.988 & 0.991 & 0.998 & 0.8 & 0.991 & 1.0 & 1.0 & 1.0 & 0.734 & 1.0 & 0.658 & 0.785 & 1.0 & 0.07 & 0.879 \\
\hline 0.95 & 0.863 & 0.797 & 0.774 & 0.811 & 0.794 & 0.988 & 0.991 & 0.998 & 0.859 & 0.991 & 1.0 & 1.0 & 1.0 & 0.789 & 1.0 & 0.658 & 0.854 & 1.0 & 0.096 & 0.881 \\
\hline 0.96 & 0.863 & 0.8 & 0.785 & 0.811 & 0.801 & 0.988 & 0.991 & 0.997 & 0.914 & 0.992 & 1.0 & 1.0 & 1.0 & 0.873 & 1.0 & 0.658 & 0.868 & 1.0 & 0.14 & 0.887 \\
\hline 0.97 & 0.863 & 0.805 & 0.794 & 0.81 & 0.803 & 0.988 & 0.991 & 0.996 & 0.982 & 0.992 & 1.0 & 1.0 & 1.0 & 0.942 & 1.0 & 0.658 & 0.909 & 1.0 & 0.227 & 0.914 \\
\hline 0.98 & 0.863 & 0.804 & 0.801 & 0.808 & 0.803 & 0.988 & 0.992 & 0.995 & 0.991 & 0.992 & 1.0 & 1.0 & 1.0 & 0.988 & 1.0 & 0.658 & 0.918 & 1.0 & 0.503 & 0.918 \\
\hline 0.99 & 0.863 & 0.806 & 0.803 & 0.81 & 0.806 & 0.988 & 0.992 & 0.993 & 0.991 & 0.992 & 1.0 & 1.0 & 1.0 & 1.0 & 1.0 & 0.658 & 0.91 & 1.0 & 0.692 & 0.915 \\
\hline 0.9999 & 0.863 & 0.809 & 0.809 & 0.809 & 0.809 & 0.988 & 0.992 & 0.992 & 0.992 & 0.992 & 1.0 & 1.0 & 1.0 & 1.0 & 1.0 & 0.658 & 0.953 & 0.955 & 0.952 & 0.953 \\
\hline 1 & 0.863 & 0.809 & 0.809 & 0.809 & 0.809 & 0.988 & 0.992 & 0.992 & 0.992 & 0.992 & 1.0 & 1.0 & 1.0 & 1.0 & 1.0 & 0.658 & 0.954 & 0.954 & 0.954 & 0.954 \\
\hline
\end{tabular}

Table 10 - Metrics registration percentage as function of the entropic index $q$, limited in the range $q \leq 1$, with 8 bits histogram binning. (A. means additive, N. means non-additive) 


\begin{tabular}{|c|c|c|c|c|c|c|c|c|c|c|c|c|c|c|c|c|c|c|c|c|}
\hline \multirow{3}{*}{$q$} & \multicolumn{5}{|c|}{ Rotation } & \multicolumn{5}{|c|}{ Scaling } & \multicolumn{5}{|c|}{ Skewness } & \multicolumn{5}{|c|}{ Translation } \\
\hline & \multirow{2}{*}{ Mattes } & \multicolumn{2}{|c|}{ Renyi } & \multicolumn{2}{|c|}{ Tsallis } & \multirow{2}{*}{ Mattes } & \multicolumn{2}{|c|}{ Renyi } & \multicolumn{2}{|c|}{ Tsallis } & \multirow{2}{*}{ Mattes } & \multicolumn{2}{|c|}{ Renyi } & \multicolumn{2}{|c|}{ Tsallis } & \multirow{2}{*}{ Mattes } & \multicolumn{2}{|c|}{ Renyi } & \multicolumn{2}{|c|}{ Tsallis } \\
\hline & & A. & N. & A. & N. & & A. & N. & A. & N. & & A. & N. & A. & N. & & A. & N. & A. & N. \\
\hline 1 & 0.863 & 0.809 & 0.809 & 0.809 & 0.809 & 0.988 & 0.992 & 0.992 & 0.992 & 0.992 & 1.0 & 1.0 & 1.0 & 1.0 & 1.0 & 0.658 & 0.954 & 0.954 & 0.954 & 0.954 \\
\hline 1.0001 & 0.863 & 0.809 & 0.809 & 0.809 & 0.809 & 0.988 & 0.992 & 0.992 & 0.992 & 0.992 & 1.0 & 1.0 & 1.0 & 1.0 & 1.0 & 0.658 & 0.954 & 0.952 & 0.956 & 0.954 \\
\hline 1.01 & 0.863 & 0.809 & 0.809 & 0.806 & 0.808 & 0.988 & 0.992 & 0.991 & 0.993 & 0.992 & 1.0 & 1.0 & 1.0 & 1.0 & 1.0 & 0.658 & 0.971 & 0.786 & 1.0 & 0.971 \\
\hline 1.02 & 0.863 & 0.81 & 0.808 & 0.803 & 0.808 & 0.988 & 0.992 & 0.991 & 0.994 & 0.992 & 1.0 & 1.0 & 0.997 & 1.0 & 1.0 & 0.658 & 0.977 & 0.697 & 1.0 & 0.977 \\
\hline 1.03 & 0.863 & 0.806 & 0.809 & 0.8 & 0.806 & 0.988 & 0.992 & 0.991 & 0.996 & 0.992 & 1.0 & 1.0 & 0.972 & 1.0 & 1.0 & 0.658 & 0.97 & 0.576 & 1.0 & 0.97 \\
\hline 1.04 & 0.863 & 0.807 & 0.807 & 0.794 & 0.804 & 0.988 & 0.992 & 0.99 & 0.998 & 0.992 & 1.0 & 1.0 & 0.929 & 1.0 & 1.0 & 0.658 & 0.962 & 0.405 & 1.0 & 0.961 \\
\hline 1.05 & 0.863 & 0.802 & 0.807 & 0.79 & 0.803 & 0.988 & 0.992 & 0.962 & 0.999 & 0.992 & 1.0 & 1.0 & 0.888 & 1.0 & 1.0 & 0.658 & 0.957 & 0.285 & 1.0 & 0.957 \\
\hline 1.06 & 0.863 & 0.802 & 0.807 & 0.791 & 0.806 & 0.988 & 0.992 & 0.894 & 1.0 & 0.992 & 1.0 & 1.0 & 0.846 & 1.0 & 1.0 & 0.658 & 0.956 & 0.226 & 1.0 & 0.956 \\
\hline 1.07 & 0.863 & 0.803 & 0.804 & 0.785 & 0.804 & 0.988 & 0.992 & 0.84 & 1.0 & 0.992 & 1.0 & 1.0 & 0.8 & 1.0 & 1.0 & 0.658 & 0.956 & 0.166 & 1.0 & 0.956 \\
\hline 1.08 & 0.863 & 0.802 & 0.803 & 0.786 & 0.802 & 0.988 & 0.992 & 0.792 & 1.0 & 0.992 & 1.0 & 1.0 & 0.761 & 1.0 & 1.0 & 0.658 & 0.955 & 0.137 & 1.0 & 0.956 \\
\hline 1.09 & 0.863 & 0.802 & 0.801 & 0.777 & 0.8 & 0.988 & 0.993 & 0.748 & 1.0 & 0.992 & 1.0 & 1.0 & 0.726 & 1.0 & 1.0 & 0.658 & 0.954 & 0.116 & 1.0 & 0.955 \\
\hline 1.1 & 0.863 & 0.798 & 0.8 & 0.774 & 0.8 & 0.988 & 0.992 & 0.706 & 1.0 & 0.992 & 1.0 & 1.0 & 0.69 & 1.0 & 1.0 & 0.658 & 0.954 & 0.102 & 1.0 & 0.955 \\
\hline 1.2 & 0.863 & 0.782 & 0.796 & 0.72 & 0.785 & 0.988 & 0.992 & 0.519 & 0.984 & 0.992 & 1.0 & 1.0 & 0.351 & 1.0 & 1.0 & 0.658 & 0.955 & 0.045 & 1.0 & 0.958 \\
\hline 1.3 & 0.863 & 0.762 & 0.772 & 0.693 & 0.767 & 0.988 & 0.992 & 0.372 & 0.737 & 0.992 & 1.0 & 1.0 & 0.195 & 1.0 & 1.0 & 0.658 & 0.955 & 0.027 & 1.0 & 0.959 \\
\hline 1.4 & 0.863 & 0.748 & 0.557 & 0.652 & 0.761 & 0.988 & 0.992 & 0.322 & 0.707 & 0.992 & 1.0 & 1.0 & 0.122 & 1.0 & 1.0 & 0.658 & 0.955 & 0.017 & 1.0 & 0.961 \\
\hline 1.5 & 0.863 & 0.735 & 0.115 & 0.634 & 0.761 & 0.988 & 0.993 & 0.284 & 0.688 & 0.987 & 1.0 & 1.0 & 0.081 & 1.0 & 1.0 & 0.658 & 0.958 & 0.011 & 1.0 & 0.963 \\
\hline 1.6 & 0.863 & 0.716 & 0.002 & 0.626 & 0.757 & 0.988 & 0.993 & 0.269 & 0.686 & 0.904 & 1.0 & 1.0 & 0.059 & 1.0 & 0.96 & 0.658 & 0.959 & 0.008 & 1.0 & 0.964 \\
\hline 1.7 & 0.863 & 0.703 & 0.001 & 0.622 & 0.746 & 0.988 & 0.994 & 0.258 & 0.679 & 0.626 & 1.0 & 1.0 & 0.045 & 1.0 & 0.836 & 0.658 & 0.96 & 0.006 & 1.0 & 0.955 \\
\hline 1.8 & 0.863 & 0.691 & 0.001 & 0.614 & 0.728 & 0.988 & 0.994 & 0.236 & 0.676 & 0.545 & 1.0 & 1.0 & 0.035 & 0.999 & 0.713 & 0.658 & 0.961 & 0.004 & 1.0 & 0.948 \\
\hline 1.9 & 0.863 & 0.677 & 0.001 & 0.623 & 0.685 & 0.988 & 0.994 & 0.227 & 0.672 & 0.51 & 1.0 & 1.0 & 0.029 & 0.999 & 0.615 & 0.658 & 0.961 & 0.004 & 1.0 & 0.929 \\
\hline 2 & 0.863 & 0.666 & 0.001 & 0.619 & 0.575 & 0.988 & 0.994 & 0.218 & 0.668 & 0.461 & 1.0 & 1.0 & 0.025 & 0.999 & 0.532 & 0.658 & 0.962 & 0.004 & 1.0 & 0.912 \\
\hline 3 & 0.863 & 0.591 & 0.001 & 0.591 & 0.001 & 0.988 & 0.992 & 0.186 & 0.67 & 0.237 & 1.0 & 1.0 & 0.013 & 0.001 & 0.063 & 0.658 & 0.963 & 0.0 & 1.0 & 0.626 \\
\hline
\end{tabular}

Table 11 - Metrics registration percentage as function of the entropic index $q$, limited in the range $q \geq 1$, with 8 bits histogram binning. (A. means additive, N. means non-additive) 


\begin{tabular}{|c|c|c|c|c|c|c|c|c|c|c|c|c|c|c|c|c|c|c|c|c|}
\hline \multirow{3}{*}{$q$} & \multicolumn{5}{|c|}{ Rotation } & \multicolumn{5}{|c|}{ Scaling } & \multicolumn{5}{|c|}{ Skewness } & \multicolumn{5}{|c|}{ Translation } \\
\hline & \multirow{2}{*}{ Mattes } & \multicolumn{2}{|c|}{ Renyi } & \multicolumn{2}{|c|}{ Tsallis } & \multirow{2}{*}{ Mattes } & \multicolumn{2}{|c|}{ Renyi } & \multicolumn{2}{|c|}{ Tsallis } & \multirow{2}{*}{ Mattes } & \multicolumn{2}{|c|}{ Renyi } & \multicolumn{2}{|c|}{ Tsallis } & \multirow{2}{*}{ Mattes } & \multicolumn{2}{|c|}{ Renyi } & \multicolumn{2}{|c|}{ Tsallis } \\
\hline & & A. & $\mathrm{N}$. & A. & $\mathrm{N}$. & & A. & $\mathrm{N}$. & A. & $\mathrm{N}$. & & A. & $\mathrm{N}$. & A. & $\mathrm{N}$. & & A. & N. & A. & $\mathrm{N}$. \\
\hline 0 & 0.863 & 0.001 & 0.023 & 0.003 & 0.003 & 0.988 & 0.019 & 0.062 & 0.011 & 0.014 & 1.0 & 0.012 & 0.017 & 0.011 & 0.014 & 0.658 & 0.017 & 0.162 & 0.01 & 0.015 \\
\hline 0.1 & 0.863 & 0.002 & 0.01 & 0.004 & 0.002 & 0.988 & 0.019 & 0.349 & 0.014 & 0.021 & 1.0 & 0.015 & 0.188 & 0.014 & 0.014 & 0.658 & 0.024 & 0.382 & 0.006 & 0.04 \\
\hline 0.2 & 0.863 & 0.009 & 0.021 & 0.006 & 0.009 & 0.988 & 0.108 & 0.664 & 0.034 & 0.087 & 1.0 & 0.02 & 0.634 & 0.016 & 0.02 & 0.658 & 0.03 & 0.659 & 0.004 & 0.036 \\
\hline 0.3 & 0.863 & 0.019 & 0.055 & 0.01 & 0.02 & 0.988 & 0.151 & 0.691 & 0.047 & 0.139 & 1.0 & 0.079 & 0.872 & 0.027 & 0.058 & 0.658 & 0.028 & 0.864 & 0.003 & 0.015 \\
\hline 0.4 & 0.863 & 0.038 & 0.127 & 0.024 & 0.034 & 0.988 & 0.236 & 0.698 & 0.104 & 0.193 & 1.0 & 0.319 & 0.977 & 0.055 & 0.22 & 0.658 & 0.024 & 0.958 & 0.002 & 0.007 \\
\hline 0.5 & 0.863 & 0.091 & 0.381 & 0.053 & 0.083 & 0.988 & 0.352 & 0.71 & 0.191 & 0.303 & 1.0 & 0.587 & 0.992 & 0.085 & 0.475 & 0.658 & 0.042 & 0.992 & 0.003 & 0.022 \\
\hline 0.6 & 0.863 & 0.438 & 0.573 & 0.153 & 0.407 & 0.988 & 0.568 & 0.745 & 0.333 & 0.526 & 1.0 & 0.849 & 0.996 & 0.118 & 0.756 & 0.658 & 0.056 & 0.997 & 0.006 & 0.038 \\
\hline 0.7 & 0.863 & 0.772 & 0.631 & 0.633 & 0.778 & 0.988 & 0.669 & 0.754 & 0.464 & 0.66 & 1.0 & 0.984 & 0.999 & 0.189 & 0.971 & 0.658 & 0.079 & 1.0 & 0.011 & 0.064 \\
\hline 0.8 & 0.863 & 0.774 & 0.646 & 0.829 & 0.775 & 0.988 & 0.832 & 0.783 & 0.603 & 0.842 & 1.0 & 1.0 & 1.0 & 0.392 & 0.999 & 0.658 & 0.157 & 1.0 & 0.022 & 0.161 \\
\hline 0.9 & 0.863 & 0.791 & 0.756 & 0.809 & 0.789 & 0.988 & 0.981 & 0.881 & 0.841 & 0.981 & 1.0 & 1.0 & 1.0 & 0.848 & 1.0 & 0.658 & 0.618 & 1.0 & 0.07 & 0.647 \\
\hline 0.91 & 0.863 & 0.796 & 0.772 & 0.817 & 0.795 & 0.988 & 0.984 & 0.929 & 0.876 & 0.984 & 1.0 & 1.0 & 1.0 & 0.877 & 1.0 & 0.658 & 0.633 & 1.0 & 0.085 & 0.658 \\
\hline 0.92 & 0.863 & 0.803 & 0.783 & 0.82 & 0.802 & 0.988 & 0.986 & 0.972 & 0.903 & 0.987 & 1.0 & 1.0 & 1.0 & 0.909 & 1.0 & 0.658 & 0.647 & 1.0 & 0.108 & 0.669 \\
\hline 0.93 & 0.863 & 0.812 & 0.787 & 0.821 & 0.812 & 0.988 & 0.989 & 0.996 & 0.945 & 0.989 & 1.0 & 1.0 & 1.0 & 0.942 & 1.0 & 0.658 & 0.661 & 1.0 & 0.133 & 0.704 \\
\hline 0.94 & 0.863 & 0.815 & 0.8 & 0.826 & 0.816 & 0.988 & 0.99 & 0.995 & 0.978 & 0.99 & 1.0 & 1.0 & 1.0 & 0.969 & 1.0 & 0.658 & 0.708 & 1.0 & 0.18 & 0.729 \\
\hline 0.95 & 0.863 & 0.82 & 0.803 & 0.826 & 0.819 & 0.988 & 0.991 & 0.995 & 0.989 & 0.991 & 1.0 & 1.0 & 1.0 & 0.989 & 1.0 & 0.658 & 0.753 & 1.0 & 0.253 & 0.761 \\
\hline 0.96 & 0.863 & 0.822 & 0.81 & 0.827 & 0.822 & 0.988 & 0.991 & 0.994 & 0.99 & 0.991 & 1.0 & 1.0 & 1.0 & 0.997 & 1.0 & 0.658 & 0.776 & 1.0 & 0.398 & 0.797 \\
\hline 0.97 & 0.863 & 0.826 & 0.824 & 0.826 & 0.825 & 0.988 & 0.991 & 0.994 & 0.99 & 0.992 & 1.0 & 1.0 & 1.0 & 1.0 & 1.0 & 0.658 & 0.81 & 0.999 & 0.506 & 0.813 \\
\hline 0.98 & 0.863 & 0.827 & 0.825 & 0.829 & 0.826 & 0.988 & 0.992 & 0.993 & 0.991 & 0.992 & 1.0 & 1.0 & 1.0 & 1.0 & 1.0 & 0.658 & 0.84 & 0.996 & 0.635 & 0.842 \\
\hline 0.99 & 0.863 & 0.828 & 0.826 & 0.831 & 0.828 & 0.988 & 0.992 & 0.993 & 0.992 & 0.992 & 1.0 & 1.0 & 1.0 & 1.0 & 1.0 & 0.658 & 0.866 & 0.972 & 0.724 & 0.866 \\
\hline 0.9999 & 0.863 & 0.832 & 0.832 & 0.832 & 0.832 & 0.988 & 0.993 & 0.993 & 0.993 & 0.993 & 1.0 & 1.0 & 1.0 & 1.0 & 1.0 & 0.658 & 0.873 & 0.873 & 0.872 & 0.873 \\
\hline 1 & 0.863 & 0.83 & 0.83 & 0.83 & 0.83 & 0.988 & 0.993 & 0.993 & 0.993 & 0.993 & 1.0 & 1.0 & 1.0 & 1.0 & 1.0 & 0.658 & 0.873 & 0.873 & 0.873 & 0.873 \\
\hline
\end{tabular}

Table 12 - Metrics registration percentage as function of the entropic index $q$, limited in the range $q \leq 1$, with 6 bits histogram binning. (A. means additive, N. means non-additive) 


\begin{tabular}{|c|c|c|c|c|c|c|c|c|c|c|c|c|c|c|c|c|c|c|c|c|}
\hline \multirow{3}{*}{$q$} & \multicolumn{5}{|c|}{ Rotation } & \multicolumn{5}{|c|}{ Scaling } & \multicolumn{5}{|c|}{ Skewness } & \multicolumn{5}{|c|}{ Translation } \\
\hline & \multirow{2}{*}{ Mattes } & \multicolumn{2}{|c|}{ Renyi } & \multicolumn{2}{|c|}{ Tsallis } & \multirow{2}{*}{ Mattes } & \multicolumn{2}{|c|}{ Renyi } & \multicolumn{2}{|c|}{ Tsallis } & \multirow{2}{*}{ Mattes } & \multicolumn{2}{|c|}{ Renyi } & \multicolumn{2}{|c|}{ Tsallis } & \multirow{2}{*}{ Mattes } & \multicolumn{2}{|c|}{ Renyi } & \multicolumn{2}{|c|}{ Tsallis } \\
\hline & & A. & N. & A. & N. & & A. & N. & A. & $\mathrm{N}$. & & A. & N. & A. & $\mathrm{N}$. & & A. & $\mathrm{N}$. & A. & $\mathrm{N}$. \\
\hline 1 & 0.863 & 0.83 & 0.83 & 0.83 & 0.83 & 0.988 & 0.993 & 0.993 & 0.993 & 0.993 & 1.0 & 1.0 & 1.0 & 1.0 & 1.0 & 0.658 & 0.873 & 0.873 & 0.873 & 0.873 \\
\hline 1.0001 & 0.863 & 0.83 & 0.83 & 0.83 & 0.83 & 0.988 & 0.993 & 0.993 & 0.993 & 0.993 & 1.0 & 1.0 & 1.0 & 1.0 & 1.0 & 0.658 & 0.873 & 0.872 & 0.874 & 0.873 \\
\hline 1.01 & 0.863 & 0.83 & 0.83 & 0.829 & 0.83 & 0.988 & 0.993 & 0.992 & 0.993 & 0.993 & 1.0 & 1.0 & 1.0 & 1.0 & 1.0 & 0.658 & 0.886 & 0.775 & 0.99 & 0.883 \\
\hline 1.02 & 0.863 & 0.831 & 0.831 & 0.828 & 0.831 & 0.988 & 0.993 & 0.992 & 0.994 & 0.992 & 1.0 & 1.0 & 1.0 & 1.0 & 1.0 & 0.658 & 0.9 & 0.667 & 1.0 & 0.899 \\
\hline 1.03 & 0.863 & 0.829 & 0.835 & 0.826 & 0.829 & 0.988 & 0.993 & 0.991 & 0.994 & 0.992 & 1.0 & 1.0 & 1.0 & 1.0 & 1.0 & 0.658 & 0.892 & 0.616 & 1.0 & 0.892 \\
\hline 1.04 & 0.863 & 0.829 & 0.837 & 0.826 & 0.832 & 0.988 & 0.993 & 0.991 & 0.995 & 0.993 & 1.0 & 1.0 & 1.0 & 1.0 & 1.0 & 0.658 & 0.901 & 0.585 & 1.0 & 0.902 \\
\hline 1.05 & 0.863 & 0.832 & 0.837 & 0.828 & 0.833 & 0.988 & 0.993 & 0.991 & 0.996 & 0.993 & 1.0 & 1.0 & 1.0 & 1.0 & 1.0 & 0.658 & 0.894 & 0.574 & 1.0 & 0.894 \\
\hline 1.06 & 0.863 & 0.831 & 0.839 & 0.826 & 0.832 & 0.988 & 0.993 & 0.991 & 0.997 & 0.992 & 1.0 & 1.0 & 1.0 & 1.0 & 1.0 & 0.658 & 0.862 & 0.578 & 1.0 & 0.862 \\
\hline 1.07 & 0.863 & 0.831 & 0.839 & 0.825 & 0.832 & 0.988 & 0.993 & 0.992 & 0.998 & 0.992 & 1.0 & 1.0 & 0.999 & 1.0 & 1.0 & 0.658 & 0.861 & 0.537 & 1.0 & 0.862 \\
\hline 1.08 & 0.863 & 0.832 & 0.842 & 0.821 & 0.832 & 0.988 & 0.992 & 0.992 & 0.999 & 0.992 & 1.0 & 1.0 & 0.997 & 1.0 & 1.0 & 0.658 & 0.862 & 0.496 & 1.0 & 0.862 \\
\hline 1.09 & 0.863 & 0.832 & 0.846 & 0.818 & 0.833 & 0.988 & 0.992 & 0.992 & 1.0 & 0.992 & 1.0 & 1.0 & 0.995 & 1.0 & 1.0 & 0.658 & 0.864 & 0.449 & 1.0 & 0.865 \\
\hline 1.1 & 0.863 & 0.834 & 0.847 & 0.822 & 0.836 & 0.988 & 0.992 & 0.992 & 1.0 & 0.993 & 1.0 & 1.0 & 0.989 & 1.0 & 1.0 & 0.658 & 0.865 & 0.414 & 1.0 & 0.866 \\
\hline 1.2 & 0.863 & 0.829 & 0.849 & 0.806 & 0.84 & 0.988 & 0.993 & 0.993 & 1.0 & 0.992 & 1.0 & 1.0 & 0.93 & 1.0 & 1.0 & 0.658 & 0.864 & 0.277 & 1.0 & 0.866 \\
\hline 1.3 & 0.863 & 0.822 & 0.851 & 0.792 & 0.833 & 0.988 & 0.993 & 0.992 & 1.0 & 0.993 & 1.0 & 1.0 & 0.875 & 1.0 & 1.0 & 0.658 & 0.862 & 0.247 & 1.0 & 0.864 \\
\hline 1.4 & 0.863 & 0.814 & 0.846 & 0.781 & 0.832 & 0.988 & 0.993 & 0.983 & 1.0 & 0.993 & 1.0 & 1.0 & 0.829 & 1.0 & 1.0 & 0.658 & 0.86 & 0.229 & 1.0 & 0.863 \\
\hline 1.5 & 0.863 & 0.809 & 0.845 & 0.773 & 0.829 & 0.988 & 0.993 & 0.881 & 1.0 & 0.993 & 1.0 & 1.0 & 0.786 & 1.0 & 1.0 & 0.658 & 0.856 & 0.21 & 1.0 & 0.863 \\
\hline 1.6 & 0.863 & 0.803 & 0.845 & 0.761 & 0.828 & 0.988 & 0.994 & 0.798 & 1.0 & 0.994 & 1.0 & 1.0 & 0.749 & 1.0 & 1.0 & 0.658 & 0.856 & 0.203 & 1.0 & 0.862 \\
\hline 1.7 & 0.863 & 0.8 & 0.844 & 0.752 & 0.826 & 0.988 & 0.994 & 0.701 & 0.998 & 0.994 & 1.0 & 1.0 & 0.714 & 1.0 & 1.0 & 0.658 & 0.855 & 0.191 & 1.0 & 0.86 \\
\hline 1.8 & 0.863 & 0.796 & 0.841 & 0.747 & 0.826 & 0.988 & 0.995 & 0.631 & 0.996 & 0.994 & 1.0 & 1.0 & 0.673 & 1.0 & 1.0 & 0.658 & 0.855 & 0.174 & 1.0 & 0.86 \\
\hline 1.9 & 0.863 & 0.794 & 0.845 & 0.734 & 0.827 & 0.988 & 0.994 & 0.59 & 0.98 & 0.994 & 1.0 & 1.0 & 0.639 & 1.0 & 1.0 & 0.658 & 0.854 & 0.153 & 1.0 & 0.86 \\
\hline 2 & 0.863 & 0.793 & 0.847 & 0.726 & 0.828 & 0.988 & 0.994 & 0.542 & 0.735 & 0.994 & 1.0 & 1.0 & 0.607 & 1.0 & 1.0 & 0.658 & 0.853 & 0.126 & 1.0 & 0.861 \\
\hline 3 & 0.863 & 0.784 & 0.801 & 0.674 & 0.833 & 0.988 & 0.994 & 0.334 & 0.712 & 0.998 & 1.0 & 1.0 & 0.0 & 1.0 & 1.0 & 0.658 & 0.851 & 0.036 & 1.0 & 0.863 \\
\hline
\end{tabular}

Table 13 - Metrics registration percentage as function of the entropic index $q$, limited in the range $q \geq 1$, with 6 bits histogram binning. (A. means additive, N. means non-additive) 


\begin{tabular}{|c|c|c|c|c|c|c|c|c|c|c|c|c|c|c|c|c|c|c|c|c|}
\hline \multirow{3}{*}{$q$} & \multicolumn{5}{|c|}{ Rotation } & \multicolumn{5}{|c|}{ Scaling } & \multicolumn{5}{|c|}{ Skewness } & \multicolumn{5}{|c|}{ Translation } \\
\hline & \multirow{2}{*}{ Mattes } & \multicolumn{2}{|c|}{ Renyi } & \multicolumn{2}{|c|}{ Tsallis } & \multirow{2}{*}{ Mattes } & \multicolumn{2}{|c|}{ Renyi } & \multicolumn{2}{|c|}{ Tsallis } & \multirow{2}{*}{ Mattes } & \multicolumn{2}{|c|}{ Renyi } & \multicolumn{2}{|c|}{ Tsallis } & \multirow{2}{*}{ Mattes } & \multicolumn{2}{|c|}{ Renyi } & \multicolumn{2}{|c|}{ Tsallis } \\
\hline & & A. & N. & A. & N. & & A. & N. & A. & N. & & A. & N. & A. & N. & & A. & N. & A. & N. \\
\hline 0 & 0.863 & 0.002 & 0.002 & 0.001 & 0.002 & 0.988 & 0.006 & 0.008 & 0.003 & 0.005 & 1.0 & 0.009 & 0.013 & 0.004 & 0.009 & 0.658 & 0.015 & 0.009 & 0.005 & 0.009 \\
\hline 0.1 & 0.863 & 0.002 & 0.005 & 0.002 & 0.002 & 0.988 & 0.013 & 0.262 & 0.006 & 0.014 & 1.0 & 0.014 & 0.198 & 0.011 & 0.014 & 0.658 & 0.034 & 0.312 & 0.008 & 0.034 \\
\hline 0.2 & 0.863 & 0.008 & 0.011 & 0.007 & 0.008 & 0.988 & 0.116 & 0.49 & 0.033 & 0.106 & 1.0 & 0.035 & 0.496 & 0.02 & 0.037 & 0.658 & 0.049 & 0.521 & 0.005 & 0.052 \\
\hline 0.3 & 0.863 & 0.023 & 0.047 & 0.019 & 0.023 & 0.988 & 0.185 & 0.692 & 0.058 & 0.181 & 1.0 & 0.308 & 0.864 & 0.063 & 0.214 & 0.658 & 0.063 & 0.736 & 0.006 & 0.054 \\
\hline 0.4 & 0.863 & 0.053 & 0.104 & 0.054 & 0.053 & 0.988 & 0.327 & 0.739 & 0.148 & 0.313 & 1.0 & 0.702 & 0.954 & 0.184 & 0.679 & 0.658 & 0.08 & 0.896 & 0.006 & 0.068 \\
\hline 0.5 & 0.863 & 0.168 & 0.261 & 0.123 & 0.169 & 0.988 & 0.546 & 0.756 & 0.32 & 0.539 & 1.0 & 0.93 & 0.961 & 0.282 & 0.91 & 0.658 & 0.114 & 0.98 & 0.009 & 0.106 \\
\hline 0.6 & 0.863 & 0.578 & 0.616 & 0.451 & 0.578 & 0.988 & 0.702 & 0.788 & 0.491 & 0.711 & 1.0 & 0.998 & 0.995 & 0.449 & 0.997 & 0.658 & 0.182 & 0.996 & 0.025 & 0.201 \\
\hline 0.7 & 0.863 & 0.808 & 0.754 & 0.804 & 0.808 & 0.988 & 0.868 & 0.815 & 0.627 & 0.86 & 1.0 & 1.0 & 0.998 & 0.708 & 1.0 & 0.658 & 0.311 & 0.998 & 0.044 & 0.38 \\
\hline 0.8 & 0.863 & 0.787 & 0.769 & 0.791 & 0.786 & 0.988 & 0.956 & 0.866 & 0.807 & 0.938 & 1.0 & 1.0 & 0.998 & 0.899 & 1.0 & 0.658 & 0.677 & 0.999 & 0.086 & 0.728 \\
\hline 0.9 & 0.863 & 0.823 & 0.822 & 0.824 & 0.821 & 0.988 & 0.996 & 0.997 & 0.994 & 0.996 & 1.0 & 1.0 & 1.0 & 0.982 & 1.0 & 0.658 & 0.743 & 0.999 & 0.241 & 0.739 \\
\hline 0.91 & 0.863 & 0.833 & 0.826 & 0.827 & 0.833 & 0.988 & 0.997 & 0.997 & 0.995 & 0.998 & 1.0 & 1.0 & 1.0 & 0.991 & 1.0 & 0.658 & 0.732 & 0.999 & 0.282 & 0.747 \\
\hline 0.92 & 0.863 & 0.838 & 0.832 & 0.834 & 0.837 & 0.988 & 0.998 & 0.998 & 0.996 & 0.998 & 1.0 & 1.0 & 1.0 & 0.994 & 1.0 & 0.658 & 0.745 & 0.998 & 0.33 & 0.765 \\
\hline 0.93 & 0.863 & 0.837 & 0.836 & 0.837 & 0.84 & 0.988 & 0.998 & 0.998 & 0.997 & 0.998 & 1.0 & 1.0 & 1.0 & 0.998 & 1.0 & 0.658 & 0.764 & 0.997 & 0.389 & 0.769 \\
\hline 0.94 & 0.863 & 0.843 & 0.845 & 0.844 & 0.843 & 0.988 & 0.998 & 0.999 & 0.997 & 0.998 & 1.0 & 1.0 & 1.0 & 1.0 & 1.0 & 0.658 & 0.767 & 0.996 & 0.443 & 0.771 \\
\hline 0.95 & 0.863 & 0.848 & 0.846 & 0.848 & 0.849 & 0.988 & 0.998 & 0.999 & 0.997 & 0.998 & 1.0 & 1.0 & 1.0 & 1.0 & 1.0 & 0.658 & 0.78 & 0.993 & 0.479 & 0.785 \\
\hline 0.96 & 0.863 & 0.847 & 0.847 & 0.851 & 0.848 & 0.988 & 0.998 & 0.999 & 0.997 & 0.998 & 1.0 & 1.0 & 1.0 & 1.0 & 1.0 & 0.658 & 0.792 & 0.988 & 0.609 & 0.794 \\
\hline 0.97 & 0.863 & 0.85 & 0.848 & 0.851 & 0.851 & 0.988 & 0.998 & 0.999 & 0.998 & 0.999 & 1.0 & 1.0 & 1.0 & 1.0 & 1.0 & 0.658 & 0.809 & 0.975 & 0.669 & 0.809 \\
\hline 0.98 & 0.863 & 0.852 & 0.851 & 0.852 & 0.852 & 0.988 & 0.999 & 0.999 & 0.998 & 0.999 & 1.0 & 1.0 & 1.0 & 1.0 & 1.0 & 0.658 & 0.817 & 0.939 & 0.698 & 0.817 \\
\hline 0.99 & 0.863 & 0.855 & 0.856 & 0.854 & 0.855 & 0.988 & 0.999 & 0.999 & 0.998 & 0.999 & 1.0 & 1.0 & 1.0 & 1.0 & 1.0 & 0.658 & 0.844 & 0.887 & 0.771 & 0.845 \\
\hline 0.9999 & 0.863 & 0.858 & 0.858 & 0.858 & 0.858 & 0.988 & 0.999 & 0.999 & 0.999 & 0.999 & 1.0 & 1.0 & 1.0 & 1.0 & 1.0 & 0.658 & 0.855 & 0.856 & 0.854 & 0.855 \\
\hline 1 & 0.863 & 0.858 & 0.858 & 0.858 & 0.858 & 0.988 & 0.999 & 0.999 & 0.999 & 0.999 & 1.0 & 1.0 & 1.0 & 1.0 & 1.0 & 0.658 & 0.856 & 0.856 & 0.856 & 0.856 \\
\hline
\end{tabular}

Table 14 - Metrics registration percentage as function of the entropic index $q$, limited in the range $q \leq 1$, with 4 bits histogram binning. (A. means additive, N. means non-additive) 


\begin{tabular}{|c|c|c|c|c|c|c|c|c|c|c|c|c|c|c|c|c|c|c|c|c|}
\hline \multirow{3}{*}{$q$} & \multicolumn{5}{|c|}{ Rotation } & \multicolumn{5}{|c|}{ Scaling } & \multicolumn{5}{|c|}{ Skewness } & \multicolumn{5}{|c|}{ Translation } \\
\hline & \multirow{2}{*}{ Mattes } & \multicolumn{2}{|c|}{ Renyi } & \multicolumn{2}{|c|}{ Tsallis } & \multirow{2}{*}{ Mattes } & \multicolumn{2}{|c|}{ Renyi } & \multicolumn{2}{|c|}{ Tsallis } & \multirow{2}{*}{ Mattes } & \multicolumn{2}{|c|}{ Renyi } & \multicolumn{2}{|c|}{ Tsallis } & \multirow{2}{*}{ Mattes } & \multicolumn{2}{|c|}{ Renyi } & \multicolumn{2}{|c|}{ Tsallis } \\
\hline & & A. & $\mathrm{N}$. & A. & N. & & A. & N. & A. & N. & & A. & N. & A. & $\mathrm{N}$. & & A. & $\mathrm{N}$. & A. & N. \\
\hline 1 & 0.863 & 0.858 & 0.858 & 0.858 & 0.858 & 0.988 & 0.999 & 0.999 & 0.999 & 0.999 & 1.0 & 1.0 & 1.0 & 1.0 & 1.0 & 0.658 & 0.856 & 0.856 & 0.856 & 0.856 \\
\hline 1.0001 & 0.863 & 0.858 & 0.858 & 0.858 & 0.858 & 0.988 & 0.999 & 0.999 & 0.999 & 0.999 & 1.0 & 1.0 & 1.0 & 1.0 & 1.0 & 0.658 & 0.856 & 0.855 & 0.858 & 0.856 \\
\hline 1.01 & 0.863 & 0.866 & 0.866 & 0.861 & 0.867 & 0.988 & 0.999 & 0.999 & 0.999 & 0.999 & 1.0 & 1.0 & 1.0 & 1.0 & 1.0 & 0.658 & 0.866 & 0.814 & 0.911 & 0.866 \\
\hline 1.02 & 0.863 & 0.869 & 0.871 & 0.863 & 0.869 & 0.988 & 0.999 & 0.998 & 0.999 & 0.999 & 1.0 & 1.0 & 1.0 & 1.0 & 1.0 & 0.658 & 0.874 & 0.745 & 0.982 & 0.874 \\
\hline 1.03 & 0.863 & 0.867 & 0.871 & 0.861 & 0.865 & 0.988 & 0.999 & 0.998 & 0.999 & 0.999 & 1.0 & 1.0 & 1.0 & 1.0 & 1.0 & 0.658 & 0.883 & 0.681 & 0.998 & 0.884 \\
\hline 1.04 & 0.863 & 0.868 & 0.875 & 0.87 & 0.869 & 0.988 & 0.999 & 0.998 & 1.0 & 0.999 & 1.0 & 1.0 & 1.0 & 1.0 & 1.0 & 0.658 & 0.886 & 0.63 & 1.0 & 0.886 \\
\hline 1.05 & 0.863 & 0.868 & 0.876 & 0.857 & 0.87 & 0.988 & 0.999 & 0.997 & 1.0 & 0.999 & 1.0 & 1.0 & 1.0 & 1.0 & 1.0 & 0.658 & 0.89 & 0.591 & 1.0 & 0.893 \\
\hline 1.06 & 0.863 & 0.868 & 0.879 & 0.856 & 0.873 & 0.988 & 0.999 & 0.998 & 1.0 & 0.999 & 1.0 & 1.0 & 1.0 & 1.0 & 1.0 & 0.658 & 0.9 & 0.564 & 1.0 & 0.902 \\
\hline 1.07 & 0.863 & 0.87 & 0.88 & 0.858 & 0.873 & 0.988 & 0.999 & 0.997 & 0.999 & 0.999 & 1.0 & 1.0 & 1.0 & 1.0 & 1.0 & 0.658 & 0.917 & 0.521 & 1.0 & 0.917 \\
\hline 1.08 & 0.863 & 0.872 & 0.879 & 0.868 & 0.871 & 0.988 & 0.999 & 0.997 & 0.999 & 0.999 & 1.0 & 1.0 & 1.0 & 1.0 & 1.0 & 0.658 & 0.913 & 0.487 & 1.0 & 0.913 \\
\hline 1.09 & 0.863 & 0.876 & 0.879 & 0.867 & 0.879 & 0.988 & 0.999 & 0.997 & 0.999 & 0.999 & 1.0 & 1.0 & 1.0 & 1.0 & 1.0 & 0.658 & 0.888 & 0.435 & 1.0 & 0.888 \\
\hline 1.1 & 0.863 & 0.876 & 0.877 & 0.868 & 0.879 & 0.988 & 0.999 & 0.996 & 0.999 & 0.999 & 1.0 & 1.0 & 1.0 & 1.0 & 1.0 & 0.658 & 0.867 & 0.409 & 1.0 & 0.867 \\
\hline 1.2 & 0.863 & 0.876 & 0.879 & 0.866 & 0.875 & 0.988 & 0.999 & 0.997 & 0.998 & 0.999 & 1.0 & 1.0 & 0.988 & 1.0 & 1.0 & 0.658 & 0.847 & 0.281 & 1.0 & 0.849 \\
\hline 1.3 & 0.863 & 0.879 & 0.882 & 0.86 & 0.882 & 0.988 & 0.999 & 0.998 & 0.998 & 0.999 & 1.0 & 1.0 & 0.971 & 1.0 & 1.0 & 0.658 & 0.844 & 0.222 & 1.0 & 0.847 \\
\hline 1.4 & 0.863 & 0.87 & 0.887 & 0.855 & 0.878 & 0.988 & 0.998 & 0.998 & 0.998 & 0.998 & 1.0 & 1.0 & 0.954 & 0.999 & 1.0 & 0.658 & 0.841 & 0.19 & 1.0 & 0.843 \\
\hline 1.5 & 0.863 & 0.871 & 0.882 & 0.851 & 0.872 & 0.988 & 0.998 & 0.998 & 0.997 & 0.999 & 1.0 & 1.0 & 0.941 & 0.999 & 1.0 & 0.658 & 0.837 & 0.175 & 1.0 & 0.841 \\
\hline 1.6 & 0.863 & 0.868 & 0.877 & 0.844 & 0.875 & 0.988 & 0.999 & 0.998 & 0.997 & 0.999 & 1.0 & 1.0 & 0.924 & 0.999 & 1.0 & 0.658 & 0.833 & 0.159 & 1.0 & 0.838 \\
\hline 1.7 & 0.863 & 0.862 & 0.874 & 0.839 & 0.873 & 0.988 & 0.999 & 0.998 & 0.998 & 0.999 & 1.0 & 1.0 & 0.904 & 0.999 & 1.0 & 0.658 & 0.831 & 0.144 & 1.0 & 0.837 \\
\hline 1.8 & 0.863 & 0.863 & 0.876 & 0.833 & 0.87 & 0.988 & 0.999 & 0.997 & 0.997 & 0.999 & 1.0 & 1.0 & 0.88 & 0.999 & 1.0 & 0.658 & 0.83 & 0.13 & 1.0 & 0.834 \\
\hline 1.9 & 0.863 & 0.859 & 0.864 & 0.826 & 0.87 & 0.988 & 0.999 & 0.938 & 0.996 & 0.999 & 1.0 & 1.0 & 0.853 & 0.999 & 1.0 & 0.658 & 0.829 & 0.115 & 1.0 & 0.834 \\
\hline 2 & 0.863 & 0.857 & 0.862 & 0.823 & 0.87 & 0.988 & 0.999 & 0.812 & 0.989 & 0.999 & 1.0 & 1.0 & 0.834 & 0.999 & 1.0 & 0.658 & 0.828 & 0.103 & 1.0 & 0.834 \\
\hline 3 & 0.863 & 0.853 & 0.821 & 0.788 & 0.869 & 0.988 & 0.999 & 0.531 & 0.741 & 1.0 & 1.0 & 1.0 & 0.552 & 0.999 & 1.0 & 0.658 & 0.826 & 0.052 & 1.0 & 0.834 \\
\hline
\end{tabular}

Table 15 - Metrics registration percentage as function of the entropic index $q$, limited in the range $q \geq 1$, with 4 bits histogram binning. (A. means additive, $\mathrm{N}$. means non-additive) 


\begin{tabular}{|c|c|c|c|c|c|c|c|c|c|c|c|c|c|c|c|c|c|c|c|c|}
\hline \multirow{3}{*}{$q$} & \multicolumn{5}{|c|}{ Rotation } & \multicolumn{5}{|c|}{ Scaling } & \multicolumn{5}{|c|}{ Skewness } & \multicolumn{5}{|c|}{ Translation } \\
\hline & \multirow{2}{*}{ Mattes } & \multicolumn{2}{|c|}{ Renyi } & \multicolumn{2}{|c|}{ Tsallis } & \multirow{2}{*}{ Mattes } & \multicolumn{2}{|c|}{ Renyi } & \multicolumn{2}{|c|}{ Tsallis } & \multirow{2}{*}{ Mattes } & \multicolumn{2}{|c|}{ Renyi } & \multicolumn{2}{|c|}{ Tsallis } & \multirow{2}{*}{ Mattes } & \multicolumn{2}{|c|}{ Renyi } & \multicolumn{2}{|c|}{ Tsallis } \\
\hline & & A. & N. & A. & N. & & A. & N. & A. & N. & & A. & $\mathrm{N}$. & A. & N. & & A. & $\mathrm{N}$. & A. & N. \\
\hline 0 & 0.863 & 0.001 & 0.001 & 0.001 & 0.001 & 0.988 & 0.001 & 0.001 & 0.001 & 0.001 & 1.0 & 0.0 & 0.0 & 0.0 & 0.0 & 0.658 & 0.001 & 0.001 & 0.001 & 0.001 \\
\hline 0.1 & 0.863 & 0.01 & 0.007 & 0.008 & 0.01 & 0.988 & 0.006 & 0.036 & 0.005 & 0.006 & 1.0 & 0.014 & 0.023 & 0.012 & 0.012 & 0.658 & 0.025 & 0.192 & 0.005 & 0.025 \\
\hline 0.2 & 0.863 & 0.003 & 0.013 & 0.003 & 0.006 & 0.988 & 0.013 & 0.234 & 0.005 & 0.012 & 1.0 & 0.019 & 0.047 & 0.017 & 0.018 & 0.658 & 0.022 & 0.24 & 0.004 & 0.018 \\
\hline 0.3 & 0.863 & 0.002 & 0.012 & 0.002 & 0.003 & 0.988 & 0.039 & 0.319 & 0.01 & 0.018 & 1.0 & 0.029 & 0.266 & 0.02 & 0.025 & 0.658 & 0.019 & 0.296 & 0.003 & 0.007 \\
\hline 0.4 & 0.863 & 0.01 & 0.02 & 0.011 & 0.01 & 0.988 & 0.074 & 0.456 & 0.032 & 0.033 & 1.0 & 0.119 & 0.534 & 0.041 & 0.087 & 0.658 & 0.009 & 0.273 & 0.003 & 0.005 \\
\hline 0.5 & 0.863 & 0.018 & 0.026 & 0.017 & 0.018 & 0.988 & 0.191 & 0.665 & 0.092 & 0.154 & 1.0 & 0.409 & 0.762 & 0.153 & 0.381 & 0.658 & 0.005 & 0.426 & 0.003 & 0.006 \\
\hline 0.6 & 0.863 & 0.046 & 0.068 & 0.043 & 0.046 & 0.988 & 0.516 & 0.803 & 0.29 & 0.482 & 1.0 & 0.613 & 0.916 & 0.312 & 0.59 & 0.658 & 0.02 & 0.446 & 0.005 & 0.018 \\
\hline 0.7 & 0.863 & 0.145 & 0.172 & 0.111 & 0.144 & 0.988 & 0.764 & 0.868 & 0.568 & 0.753 & 1.0 & 0.764 & 0.969 & 0.555 & 0.754 & 0.658 & 0.058 & 0.619 & 0.015 & 0.052 \\
\hline 0.8 & 0.863 & 0.349 & 0.316 & 0.44 & 0.349 & 0.988 & 0.865 & 0.941 & 0.837 & 0.864 & 1.0 & 0.976 & 0.994 & 0.776 & 0.974 & 0.658 & 0.09 & 0.729 & 0.023 & 0.088 \\
\hline 0.9 & 0.863 & 0.789 & 0.77 & 0.789 & 0.789 & 0.988 & 0.926 & 0.962 & 0.925 & 0.926 & 1.0 & 0.999 & 0.996 & 0.993 & 0.999 & 0.658 & 0.152 & 0.81 & 0.039 & 0.152 \\
\hline 0.91 & 0.863 & 0.816 & 0.81 & 0.817 & 0.815 & 0.988 & 0.931 & 0.962 & 0.933 & 0.93 & 1.0 & 0.999 & 0.997 & 0.997 & 0.999 & 0.658 & 0.152 & 0.827 & 0.042 & 0.152 \\
\hline 0.92 & 0.863 & 0.833 & 0.842 & 0.836 & 0.833 & 0.988 & 0.939 & 0.963 & 0.939 & 0.939 & 1.0 & 0.998 & 0.997 & 0.999 & 0.998 & 0.658 & 0.171 & 0.835 & 0.051 & 0.171 \\
\hline 0.93 & 0.863 & 0.849 & 0.855 & 0.847 & 0.849 & 0.988 & 0.944 & 0.967 & 0.944 & 0.944 & 1.0 & 0.998 & 0.997 & 1.0 & 0.998 & 0.658 & 0.183 & 0.83 & 0.068 & 0.183 \\
\hline 0.94 & 0.863 & 0.858 & 0.862 & 0.856 & 0.858 & 0.988 & 0.948 & 0.968 & 0.949 & 0.948 & 1.0 & 0.998 & 0.997 & 1.0 & 0.998 & 0.658 & 0.231 & 0.808 & 0.08 & 0.233 \\
\hline 0.95 & 0.863 & 0.866 & 0.867 & 0.862 & 0.866 & 0.988 & 0.953 & 0.97 & 0.955 & 0.953 & 1.0 & 0.998 & 0.997 & 1.0 & 0.998 & 0.658 & 0.416 & 0.841 & 0.105 & 0.413 \\
\hline 0.96 & 0.863 & 0.869 & 0.87 & 0.871 & 0.869 & 0.988 & 0.959 & 0.971 & 0.958 & 0.959 & 1.0 & 0.998 & 0.997 & 0.999 & 0.998 & 0.658 & 0.448 & 0.831 & 0.141 & 0.448 \\
\hline 0.97 & 0.863 & 0.868 & 0.868 & 0.873 & 0.868 & 0.988 & 0.963 & 0.972 & 0.963 & 0.963 & 1.0 & 0.998 & 0.998 & 0.999 & 0.998 & 0.658 & 0.499 & 0.786 & 0.21 & 0.504 \\
\hline 0.98 & 0.863 & 0.87 & 0.869 & 0.871 & 0.87 & 0.988 & 0.967 & 0.973 & 0.966 & 0.967 & 1.0 & 0.998 & 0.998 & 0.998 & 0.998 & 0.658 & 0.505 & 0.78 & 0.235 & 0.505 \\
\hline 0.99 & 0.863 & 0.871 & 0.87 & 0.87 & 0.871 & 0.988 & 0.97 & 0.974 & 0.97 & 0.971 & 1.0 & 0.998 & 0.998 & 0.998 & 0.998 & 0.658 & 0.525 & 0.705 & 0.443 & 0.525 \\
\hline 0.9999 & 0.863 & 0.874 & 0.874 & 0.874 & 0.874 & 0.988 & 0.974 & 0.974 & 0.974 & 0.974 & 1.0 & 0.998 & 0.998 & 0.998 & 0.998 & 0.658 & 0.563 & 0.564 & 0.561 & 0.563 \\
\hline 1 & 0.863 & 0.874 & 0.874 & 0.874 & 0.874 & 0.988 & 0.974 & 0.974 & 0.974 & 0.974 & 1.0 & 0.998 & 0.998 & 0.998 & 0.998 & 0.658 & 0.563 & 0.563 & 0.563 & 0.563 \\
\hline
\end{tabular}

Table 16 - Metrics registration percentage as function of the entropic index $q$, limited in the range $q \leq 1$, with 2 bits histogram binning. (A. means additive, N. means non-additive) 


\begin{tabular}{|c|c|c|c|c|c|c|c|c|c|c|c|c|c|c|c|c|c|c|c|c|}
\hline \multirow{3}{*}{$q$} & \multicolumn{5}{|c|}{ Rotation } & \multicolumn{5}{|c|}{ Scaling } & \multicolumn{5}{|c|}{ Skewness } & \multicolumn{5}{|c|}{ Translation } \\
\hline & \multirow{2}{*}{ Mattes } & \multicolumn{2}{|c|}{ Renyi } & \multicolumn{2}{|c|}{ Tsallis } & \multirow{2}{*}{ Mattes } & \multicolumn{2}{|c|}{ Renyi } & \multicolumn{2}{|c|}{ Tsallis } & \multirow{2}{*}{ Mattes } & \multicolumn{2}{|c|}{ Renyi } & \multicolumn{2}{|c|}{ Tsallis } & \multirow{2}{*}{ Mattes } & \multicolumn{2}{|c|}{ Renyi } & \multicolumn{2}{|c|}{ Tsallis } \\
\hline & & A. & N. & A. & N. & & A. & N. & A. & N. & & A. & N. & A. & N. & & A. & N. & A. & N. \\
\hline 1 & 0.863 & 0.874 & 0.874 & 0.874 & 0.874 & 0.988 & 0.974 & 0.974 & 0.974 & 0.974 & 1.0 & 0.998 & 0.998 & 0.998 & 0.998 & 0.658 & 0.563 & 0.563 & 0.563 & 0.563 \\
\hline 1.0001 & 0.863 & 0.874 & 0.874 & 0.874 & 0.874 & 0.988 & 0.974 & 0.974 & 0.974 & 0.974 & 1.0 & 0.998 & 0.998 & 0.998 & 0.998 & 0.658 & 0.563 & 0.557 & 0.565 & 0.563 \\
\hline 1.01 & 0.863 & 0.874 & 0.875 & 0.876 & 0.874 & 0.988 & 0.978 & 0.978 & 0.98 & 0.978 & 1.0 & 0.998 & 0.998 & 0.998 & 0.998 & 0.658 & 0.573 & 0.492 & 0.754 & 0.573 \\
\hline 1.02 & 0.863 & 0.878 & 0.878 & 0.877 & 0.878 & 0.988 & 0.983 & 0.979 & 0.986 & 0.983 & 1.0 & 0.998 & 0.998 & 0.997 & 0.998 & 0.658 & 0.611 & 0.473 & 0.867 & 0.611 \\
\hline 1.03 & 0.863 & 0.878 & 0.877 & 0.875 & 0.878 & 0.988 & 0.985 & 0.979 & 0.989 & 0.985 & 1.0 & 0.998 & 0.998 & 0.997 & 0.998 & 0.658 & 0.638 & 0.524 & 0.917 & 0.638 \\
\hline 1.04 & 0.863 & 0.876 & 0.879 & 0.874 & 0.876 & 0.988 & 0.986 & 0.98 & 0.991 & 0.986 & 1.0 & 0.998 & 0.998 & 0.997 & 0.998 & 0.658 & 0.645 & 0.518 & 0.939 & 0.634 \\
\hline 1.05 & 0.863 & 0.877 & 0.879 & 0.875 & 0.877 & 0.988 & 0.989 & 0.982 & 0.991 & 0.989 & 1.0 & 0.998 & 0.998 & 0.997 & 0.998 & 0.658 & 0.635 & 0.511 & 0.954 & 0.635 \\
\hline 1.06 & 0.863 & 0.877 & 0.878 & 0.875 & 0.877 & 0.988 & 0.992 & 0.986 & 0.992 & 0.991 & 1.0 & 0.998 & 0.998 & 0.997 & 0.998 & 0.658 & 0.592 & 0.51 & 0.967 & 0.591 \\
\hline 1.07 & 0.863 & 0.877 & 0.881 & 0.875 & 0.877 & 0.988 & 0.993 & 0.989 & 0.991 & 0.993 & 1.0 & 0.998 & 0.999 & 0.997 & 0.998 & 0.658 & 0.616 & 0.512 & 0.97 & 0.615 \\
\hline 1.08 & 0.863 & 0.877 & 0.88 & 0.875 & 0.877 & 0.988 & 0.994 & 0.993 & 0.991 & 0.994 & 1.0 & 0.998 & 0.999 & 0.997 & 0.998 & 0.658 & 0.629 & 0.516 & 0.972 & 0.627 \\
\hline 1.09 & 0.863 & 0.877 & 0.88 & 0.874 & 0.877 & 0.988 & 0.994 & 0.994 & 0.99 & 0.994 & 1.0 & 0.997 & 0.999 & 0.997 & 0.998 & 0.658 & 0.641 & 0.511 & 0.975 & 0.642 \\
\hline 1.1 & 0.863 & 0.876 & 0.882 & 0.876 & 0.876 & 0.988 & 0.994 & 0.994 & 0.99 & 0.994 & 1.0 & 0.997 & 0.999 & 0.996 & 0.998 & 0.658 & 0.643 & 0.525 & 0.98 & 0.643 \\
\hline 1.2 & 0.863 & 0.876 & 0.878 & 0.874 & 0.876 & 0.988 & 0.994 & 0.997 & 0.988 & 0.994 & 1.0 & 0.997 & 0.999 & 0.995 & 0.997 & 0.658 & 0.665 & 0.49 & 0.988 & 0.666 \\
\hline 1.3 & 0.863 & 0.876 & 0.88 & 0.876 & 0.876 & 0.988 & 0.993 & 0.997 & 0.987 & 0.994 & 1.0 & 0.997 & 1.0 & 0.977 & 0.997 & 0.658 & 0.669 & 0.479 & 0.989 & 0.668 \\
\hline 1.4 & 0.863 & 0.876 & 0.879 & 0.876 & 0.876 & 0.988 & 0.993 & 0.997 & 0.986 & 0.993 & 1.0 & 0.996 & 1.0 & 0.958 & 0.997 & 0.658 & 0.671 & 0.47 & 0.991 & 0.667 \\
\hline 1.5 & 0.863 & 0.877 & 0.877 & 0.877 & 0.877 & 0.988 & 0.993 & 0.997 & 0.986 & 0.993 & 1.0 & 0.996 & 1.0 & 0.958 & 0.997 & 0.658 & 0.67 & 0.441 & 0.993 & 0.669 \\
\hline 1.6 & 0.863 & 0.878 & 0.872 & 0.877 & 0.878 & 0.988 & 0.992 & 0.997 & 0.985 & 0.993 & 1.0 & 0.996 & 1.0 & 0.958 & 0.996 & 0.658 & 0.671 & 0.41 & 0.993 & 0.67 \\
\hline 1.7 & 0.863 & 0.879 & 0.873 & 0.88 & 0.879 & 0.988 & 0.991 & 0.997 & 0.985 & 0.993 & 1.0 & 0.996 & 1.0 & 0.958 & 0.996 & 0.658 & 0.67 & 0.386 & 0.993 & 0.672 \\
\hline 1.8 & 0.863 & 0.879 & 0.873 & 0.875 & 0.877 & 0.988 & 0.991 & 0.997 & 0.986 & 0.993 & 1.0 & 0.996 & 1.0 & 0.958 & 0.996 & 0.658 & 0.67 & 0.366 & 0.993 & 0.67 \\
\hline 1.9 & 0.863 & 0.877 & 0.873 & 0.876 & 0.878 & 0.988 & 0.991 & 0.997 & 0.98 & 0.993 & 1.0 & 0.984 & 1.0 & 0.957 & 0.996 & 0.658 & 0.668 & 0.326 & 0.993 & 0.669 \\
\hline 2 & 0.863 & 0.877 & 0.879 & 0.876 & 0.877 & 0.988 & 0.991 & 0.997 & 0.979 & 0.993 & 1.0 & 0.984 & 1.0 & 0.957 & 0.996 & 0.658 & 0.669 & 0.301 & 0.994 & 0.669 \\
\hline 3 & 0.863 & 0.876 & 0.877 & 0.878 & 0.871 & 0.988 & 0.99 & 0.993 & 0.972 & 0.993 & 1.0 & 0.982 & 0.995 & 0.957 & 0.996 & 0.658 & 0.669 & 0.12 & 0.996 & 0.67 \\
\hline
\end{tabular}

Table 17 - Metrics registration percentage as function of the entropic index $q$, limited in the range $q \geq 1$, with 2 bits histogram binning. (A. means additive, N. means non-additive) 


\begin{tabular}{|c|c|c|c|c|c|c|c|c|c|c|c|c|c|c|c|c|c|c|c|c|}
\hline \multirow{3}{*}{$q$} & \multicolumn{5}{|c|}{ Rotation } & \multicolumn{5}{|c|}{ Scaling } & \multicolumn{5}{|c|}{ Skewness } & \multicolumn{5}{|c|}{ Translation } \\
\hline & \multirow{2}{*}{ Mattes } & \multicolumn{2}{|c|}{ Renyi } & \multicolumn{2}{|c|}{ Tsallis } & \multirow{2}{*}{ Mattes } & \multicolumn{2}{|c|}{ Renyi } & \multicolumn{2}{|c|}{ Tsallis } & \multirow{2}{*}{ Mattes } & \multicolumn{2}{|c|}{ Renyi } & \multicolumn{2}{|c|}{ Tsallis } & \multirow{2}{*}{ Mattes } & \multicolumn{2}{|c|}{ Renyi } & \multicolumn{2}{|c|}{ Tsallis } \\
\hline & & A. & $\mathrm{N}$. & A. & $\mathrm{N}$. & & A. & $\mathrm{N}$. & A. & $\mathrm{N}$. & & A. & $\mathrm{N}$. & A. & $\mathrm{N}$. & & A. & N. & A. & $\mathrm{N}$. \\
\hline 0 & 0.863 & 0.0 & 0.0 & 0.0 & 0.0 & 0.988 & 0.0 & 0.0 & 0.0 & 0.0 & 1.0 & 0.0 & 0.0 & 0.0 & 0.0 & 0.658 & 0.0 & 0.0 & 0.0 & 0.0 \\
\hline 0.1 & 0.863 & 0.001 & 0.002 & 0.001 & 0.001 & 0.988 & 0.002 & 0.06 & 0.002 & 0.003 & 1.0 & 0.003 & 0.008 & 0.002 & 0.002 & 0.658 & 0.006 & 0.043 & 0.002 & 0.004 \\
\hline 0.2 & 0.863 & 0.001 & 0.002 & 0.001 & 0.001 & 0.988 & 0.003 & 0.009 & 0.002 & 0.003 & 1.0 & 0.003 & 0.009 & 0.002 & 0.003 & 0.658 & 0.004 & 0.027 & 0.002 & 0.004 \\
\hline 0.3 & 0.863 & 0.001 & 0.001 & 0.001 & 0.001 & 0.988 & 0.003 & 0.005 & 0.003 & 0.003 & 1.0 & 0.003 & 0.006 & 0.003 & 0.003 & 0.658 & 0.004 & 0.013 & 0.002 & 0.004 \\
\hline 0.4 & 0.863 & 0.001 & 0.001 & 0.001 & 0.001 & 0.988 & 0.003 & 0.003 & 0.004 & 0.003 & 1.0 & 0.004 & 0.005 & 0.004 & 0.004 & 0.658 & 0.003 & 0.004 & 0.002 & 0.003 \\
\hline 0.5 & 0.863 & 0.002 & 0.002 & 0.001 & 0.002 & 0.988 & 0.005 & 0.005 & 0.005 & 0.005 & 1.0 & 0.007 & 0.007 & 0.007 & 0.007 & 0.658 & 0.003 & 0.003 & 0.002 & 0.003 \\
\hline 0.6 & 0.863 & 0.003 & 0.003 & 0.003 & 0.003 & 0.988 & 0.01 & 0.009 & 0.009 & 0.01 & 1.0 & 0.013 & 0.013 & 0.013 & 0.013 & 0.658 & 0.002 & 0.005 & 0.002 & 0.002 \\
\hline 0.7 & 0.863 & 0.007 & 0.008 & 0.007 & 0.007 & 0.988 & 0.022 & 0.022 & 0.021 & 0.021 & 1.0 & 0.037 & 0.039 & 0.036 & 0.037 & 0.658 & 0.002 & 0.004 & 0.002 & 0.002 \\
\hline 0.8 & 0.863 & 0.041 & 0.041 & 0.041 & 0.041 & 0.988 & 0.172 & 0.179 & 0.162 & 0.172 & 1.0 & 0.16 & 0.153 & 0.163 & 0.16 & 0.658 & 0.008 & 0.034 & 0.006 & 0.008 \\
\hline 0.9 & 0.863 & 0.176 & 0.179 & 0.173 & 0.176 & 0.988 & 0.4 & 0.502 & 0.321 & 0.4 & 1.0 & 0.636 & 0.678 & 0.639 & 0.636 & 0.658 & 0.036 & 0.414 & 0.022 & 0.036 \\
\hline 0.91 & 0.863 & 0.198 & 0.2 & 0.198 & 0.198 & 0.988 & 0.439 & 0.569 & 0.352 & 0.439 & 1.0 & 0.692 & 0.767 & 0.681 & 0.692 & 0.658 & 0.337 & 0.417 & 0.028 & 0.33 \\
\hline 0.92 & 0.863 & 0.216 & 0.22 & 0.218 & 0.216 & 0.988 & 0.502 & 0.614 & 0.39 & 0.502 & 1.0 & 0.772 & 0.847 & 0.731 & 0.772 & 0.658 & 0.338 & 0.441 & 0.039 & 0.337 \\
\hline 0.93 & 0.863 & 0.235 & 0.239 & 0.238 & 0.235 & 0.988 & 0.552 & 0.737 & 0.46 & 0.552 & 1.0 & 0.848 & 0.906 & 0.792 & 0.848 & 0.658 & 0.332 & 0.473 & 0.054 & 0.331 \\
\hline 0.94 & 0.863 & 0.256 & 0.263 & 0.253 & 0.256 & 0.988 & 0.6 & 0.769 & 0.529 & 0.6 & 1.0 & 0.911 & 0.943 & 0.887 & 0.911 & 0.658 & 0.33 & 0.467 & 0.105 & 0.333 \\
\hline 0.95 & 0.863 & 0.287 & 0.293 & 0.284 & 0.287 & 0.988 & 0.664 & 0.87 & 0.57 & 0.664 & 1.0 & 0.948 & 0.967 & 0.934 & 0.948 & 0.658 & 0.411 & 0.489 & 0.107 & 0.412 \\
\hline 0.96 & 0.863 & 0.311 & 0.331 & 0.312 & 0.311 & 0.988 & 0.721 & 0.877 & 0.607 & 0.721 & 1.0 & 0.97 & 0.983 & 0.968 & 0.97 & 0.658 & 0.488 & 0.554 & 0.224 & 0.489 \\
\hline 0.97 & 0.863 & 0.346 & 0.359 & 0.339 & 0.346 & 0.988 & 0.731 & 0.885 & 0.617 & 0.731 & 1.0 & 0.983 & 0.988 & 0.98 & 0.983 & 0.658 & 0.506 & 0.559 & 0.378 & 0.508 \\
\hline 0.98 & 0.863 & 0.413 & 0.442 & 0.4 & 0.413 & 0.988 & 0.701 & 0.877 & 0.629 & 0.701 & 1.0 & 0.988 & 0.989 & 0.989 & 0.988 & 0.658 & 0.606 & 0.582 & 0.427 & 0.708 \\
\hline 0.99 & 0.863 & 0.47 & 0.466 & 0.455 & 0.47 & 0.988 & 0.748 & 0.822 & 0.646 & 0.748 & 1.0 & 0.99 & 0.99 & 0.99 & 0.99 & 0.658 & 0.627 & 0.641 & 0.496 & 0.64 \\
\hline 0.9999 & 0.863 & 0.531 & 0.531 & 0.531 & 0.531 & 0.988 & 0.847 & 0.855 & 0.847 & 0.847 & 1.0 & 0.99 & 0.99 & 0.99 & 0.99 & 0.658 & 0.661 & 0.663 & 0.595 & 0.665 \\
\hline 1 & 0.863 & 0.531 & 0.531 & 0.531 & 0.531 & 0.988 & 0.847 & 0.847 & 0.847 & 0.847 & 1.0 & 0.99 & 0.99 & 0.99 & 0.99 & 0.658 & 0.643 & 0.643 & 0.642 & 0.642 \\
\hline
\end{tabular}

Table 18 - Metrics registration percentage as function of the entropic index $q$, limited in the range $q \leq 1$, with 1 bit histogram binning. (A. means additive, N. means non-additive) 


\begin{tabular}{|c|c|c|c|c|c|c|c|c|c|c|c|c|c|c|c|c|c|c|c|c|}
\hline \multirow{3}{*}{$q$} & \multicolumn{5}{|c|}{ Rotation } & \multicolumn{5}{|c|}{ Scaling } & \multicolumn{5}{|c|}{ Skewness } & \multicolumn{5}{|c|}{ Translation } \\
\hline & \multirow{2}{*}{ Mattes } & \multicolumn{2}{|c|}{ Renyi } & \multicolumn{2}{|c|}{ Tsallis } & \multirow{2}{*}{ Mattes } & \multicolumn{2}{|c|}{ Renyi } & \multicolumn{2}{|c|}{ Tsallis } & \multirow{2}{*}{ Mattes } & \multicolumn{2}{|c|}{ Renyi } & \multicolumn{2}{|c|}{ Tsallis } & \multirow{2}{*}{ Mattes } & \multicolumn{2}{|c|}{ Renyi } & \multicolumn{2}{|c|}{ Tsallis } \\
\hline & & A. & N. & A. & $\mathrm{N}$. & & A. & $\mathrm{N}$. & A. & $\mathrm{N}$. & & A. & $\mathrm{N}$. & A. & $\mathrm{N}$. & & A. & $\mathrm{N}$. & A. & $\mathrm{N}$. \\
\hline 1 & 0.863 & 0.531 & 0.531 & 0.531 & 0.531 & 0.988 & 0.847 & 0.847 & 0.847 & 0.847 & 1.0 & 0.99 & 0.99 & 0.99 & 0.99 & 0.658 & 0.643 & 0.643 & 0.642 & 0.642 \\
\hline 1.0001 & 0.863 & 0.531 & 0.531 & 0.531 & 0.531 & 0.988 & 0.847 & 0.847 & 0.848 & 0.847 & 1.0 & 0.99 & 0.99 & 0.991 & 0.99 & 0.658 & 0.664 & 0.667 & 0.574 & 0.664 \\
\hline 1.01 & 0.863 & 0.581 & 0.579 & 0.595 & 0.581 & 0.988 & 0.853 & 0.744 & 0.889 & 0.853 & 1.0 & 0.99 & 0.991 & 0.99 & 0.99 & 0.658 & 0.697 & 0.409 & 0.644 & 0.695 \\
\hline 1.02 & 0.863 & 0.643 & 0.813 & 0.651 & 0.643 & 0.988 & 0.857 & 0.731 & 0.939 & 0.857 & 1.0 & 0.99 & 0.99 & 0.99 & 0.99 & 0.658 & 0.456 & 0.403 & 0.714 & 0.459 \\
\hline 1.03 & 0.863 & 0.781 & 0.841 & 0.716 & 0.78 & 0.988 & 0.765 & 0.773 & 0.962 & 0.765 & 1.0 & 0.991 & 0.99 & 0.99 & 0.991 & 0.658 & 0.449 & 0.405 & 0.781 & 0.456 \\
\hline 1.04 & 0.863 & 0.834 & 0.848 & 0.785 & 0.834 & 0.988 & 0.796 & 0.779 & 0.967 & 0.796 & 1.0 & 0.99 & 0.99 & 0.988 & 0.99 & 0.658 & 0.438 & 0.42 & 0.77 & 0.439 \\
\hline 1.05 & 0.863 & 0.848 & 0.85 & 0.81 & 0.848 & 0.988 & 0.8 & 0.786 & 0.968 & 0.8 & 1.0 & 0.99 & 0.99 & 0.985 & 0.99 & 0.658 & 0.443 & 0.417 & 0.791 & 0.444 \\
\hline 1.06 & 0.863 & 0.852 & 0.859 & 0.814 & 0.852 & 0.988 & 0.801 & 0.787 & 0.967 & 0.801 & 1.0 & 0.99 & 0.99 & 0.984 & 0.99 & 0.658 & 0.449 & 0.424 & 0.8 & 0.452 \\
\hline 1.07 & 0.863 & 0.854 & 0.862 & 0.822 & 0.854 & 0.988 & 0.804 & 0.784 & 0.967 & 0.804 & 1.0 & 0.986 & 0.99 & 0.983 & 0.986 & 0.658 & 0.447 & 0.421 & 0.808 & 0.449 \\
\hline 1.08 & 0.863 & 0.855 & 0.862 & 0.824 & 0.855 & 0.988 & 0.804 & 0.784 & 0.966 & 0.804 & 1.0 & 0.986 & 0.99 & 0.982 & 0.986 & 0.658 & 0.456 & 0.429 & 0.816 & 0.458 \\
\hline 1.09 & 0.863 & 0.856 & 0.86 & 0.826 & 0.855 & 0.988 & 0.805 & 0.785 & 0.967 & 0.805 & 1.0 & 0.986 & 0.99 & 0.982 & 0.986 & 0.658 & 0.451 & 0.424 & 0.828 & 0.461 \\
\hline 1.1 & 0.863 & 0.855 & 0.861 & 0.826 & 0.855 & 0.988 & 0.805 & 0.785 & 0.967 & 0.805 & 1.0 & 0.984 & 0.99 & 0.982 & 0.984 & 0.658 & 0.459 & 0.433 & 0.806 & 0.458 \\
\hline 1.2 & 0.863 & 0.855 & 0.862 & 0.824 & 0.855 & 0.988 & 0.807 & 0.788 & 0.965 & 0.806 & 1.0 & 0.981 & 0.987 & 0.98 & 0.981 & 0.658 & 0.462 & 0.435 & 0.795 & 0.458 \\
\hline 1.3 & 0.863 & 0.854 & 0.862 & 0.834 & 0.854 & 0.988 & 0.806 & 0.787 & 0.966 & 0.806 & 1.0 & 0.981 & 0.985 & 0.977 & 0.981 & 0.658 & 0.46 & 0.429 & 0.813 & 0.462 \\
\hline 1.4 & 0.863 & 0.854 & 0.862 & 0.837 & 0.854 & 0.988 & 0.807 & 0.788 & 0.966 & 0.807 & 1.0 & 0.98 & 0.984 & 0.977 & 0.981 & 0.658 & 0.457 & 0.427 & 0.805 & 0.457 \\
\hline 1.5 & 0.863 & 0.854 & 0.864 & 0.837 & 0.854 & 0.988 & 0.806 & 0.786 & 0.967 & 0.806 & 1.0 & 0.978 & 0.984 & 0.977 & 0.978 & 0.658 & 0.454 & 0.421 & 0.803 & 0.46 \\
\hline 1.6 & 0.863 & 0.854 & 0.865 & 0.84 & 0.854 & 0.988 & 0.806 & 0.785 & 0.967 & 0.806 & 1.0 & 0.978 & 0.984 & 0.976 & 0.978 & 0.658 & 0.454 & 0.423 & 0.835 & 0.462 \\
\hline 1.7 & 0.863 & 0.864 & 0.866 & 0.84 & 0.864 & 0.988 & 0.807 & 0.789 & 0.967 & 0.807 & 1.0 & 0.978 & 0.984 & 0.976 & 0.978 & 0.658 & 0.455 & 0.423 & 0.825 & 0.457 \\
\hline 1.8 & 0.863 & 0.864 & 0.866 & 0.837 & 0.864 & 0.988 & 0.807 & 0.789 & 0.966 & 0.807 & 1.0 & 0.978 & 0.984 & 0.975 & 0.978 & 0.658 & 0.458 & 0.417 & 0.812 & 0.457 \\
\hline 1.9 & 0.863 & 0.864 & 0.867 & 0.829 & 0.864 & 0.988 & 0.807 & 0.786 & 0.967 & 0.807 & 1.0 & 0.978 & 0.984 & 0.975 & 0.978 & 0.658 & 0.457 & 0.416 & 0.827 & 0.46 \\
\hline 2 & 0.863 & 0.864 & 0.867 & 0.827 & 0.864 & 0.988 & 0.807 & 0.787 & 0.967 & 0.807 & 1.0 & 0.978 & 0.984 & 0.975 & 0.978 & 0.658 & 0.455 & 0.407 & 0.805 & 0.459 \\
\hline 3 & 0.863 & 0.864 & 0.868 & 0.828 & 0.864 & 0.988 & 0.807 & 0.771 & 0.969 & 0.807 & 1.0 & 0.978 & 0.987 & 0.96 & 0.978 & 0.658 & 0.46 & 0.392 & 0.829 & 0.453 \\
\hline
\end{tabular}

Table 19 - Metrics registration percentage as function of the entropic index $q$, limited in the range $q \leq 1$, with 1 bit histogram binning. (A. means additive, N. means non-additive) 



\section{APPENDIX B - Monte Carlo}

\section{B.1 Monte Carlo Results}

\section{B.1.1 Same image - High Quality}

Those trials were made using the same image for the fixed and moving images. This gives a benchmark on how the metric tracks geometric transformation on the image.

Those tests used the image 172635_3T_T1w_MPR1.nii with 10.000 trials on each entropic parameter for Shannon and Tsallis and 10.000 trials for Mattes.

For a full log of the trials please check the files: paper_mattes_fixed_T1xT1_MT8_10k.log, paper_translation_10000.

Table 20 shows the parsed results, with Mattes having a poor performance compared to Shannon and Tsallis. Figure 51 shows the success rate as a function of the loss threshold, i.e., the registering trial's final position.

Figure 51 - Monte Carlo results for translation with same image as fixed and moving, using the HCP 172635_3T_T1w_MPR1.nii

Success Rate per Distance Threshold in Translation with Nearest Neighbor (HCP T1 1 T1)

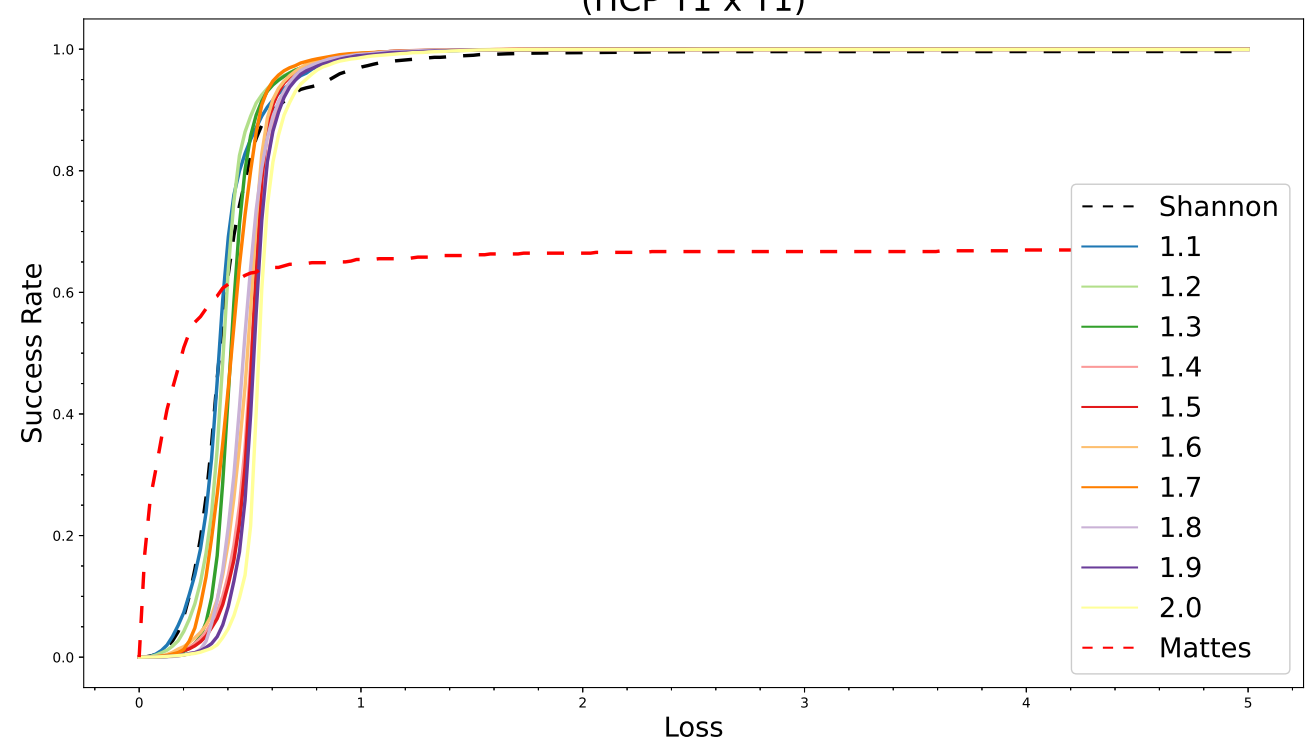

Source: author. 
Table 20 - Monte Carlo results for translation with same image as fixed and moving, using the HCP 172635_3T_T1w_MPR1.nii

\begin{tabular}{lccccrrr}
\hline Metric & Q & Binning & $<1.0$ & $<3.0$ & $<5.0$ & Mean & Stddev \\
\hline Mattes & 1.0 & Yes & $65.40 \%$ & $66.71 \%$ & $67.10 \%$ & 46.90 & 72.25 \\
Shannon & 1.0 & No & $97.05 \%$ & $99.61 \%$ & $99.62 \%$ & 0.68 & 5.38 \\
Tsallis & 1.1 & No & $98.83 \%$ & $99.97 \%$ & $99.97 \%$ & 0.44 & 3.32 \\
Tsallis & 1.2 & No & $98.97 \%$ & $99.97 \%$ & $99.97 \%$ & 0.45 & 3.31 \\
Tsallis & 1.3 & No & $99.29 \%$ & $99.97 \%$ & $99.97 \%$ & 0.49 & 3.31 \\
Tsallis & 1.4 & No & $98.87 \%$ & $99.97 \%$ & $99.97 \%$ & 0.57 & 3.31 \\
Tsallis & 1.5 & No & $99.19 \%$ & $99.97 \%$ & $99.97 \%$ & 0.56 & 3.31 \\
Tsallis & 1.6 & No & $99.28 \%$ & $99.97 \%$ & $99.97 \%$ & 0.54 & 3.31 \\
Tsallis & 1.7 & No & $99.33 \%$ & $99.97 \%$ & $99.97 \%$ & 0.48 & 3.31 \\
Tsallis & 1.8 & No & $99.15 \%$ & $99.97 \%$ & $99.97 \%$ & 0.54 & 3.31 \\
Tsallis & 1.9 & No & $98.93 \%$ & $99.97 \%$ & $99.97 \%$ & 0.58 & 3.31 \\
Tsallis & 2.0 & No & $98.61 \%$ & $99.97 \%$ & $99.97 \%$ & 0.61 & 3.31 \\
\hline
\end{tabular}

Source: author.

\section{B.1.2 Same image - Low Quality}

Those trials were made using the same image for the fixed and moving images. This gives a benchmark on how the metric tracks geometric transformation on the image.

Those tests used the image N2_T1.nrrd with 10.000 trials on each entropic parameter for Shannon and Tsallis and 1.000 trials for Mattes using the High Quality only for comparison.

For a full log of the trials please check the files: paper_mattes_fixed_N2_T1xT1.log, montecarlo_ITK_translation_fixed.log.

Table 21 shows the parsed results, with Mattes having a poor performance compared to Shannon and Tsallis. Figure 52 shows the success rate as a function of the loss threshold, i.e., the final position in the registering trial.

\section{B.1.3 Same patient - T1 x T2 - High Resolution}

Those trials were made using the same patient but with $\mathrm{T} 1$ and $\mathrm{T} 2$ as fixed and moving images, respectively. This gives a benchmark on how the metric tracks geometric transformation on the image in intermodality.

Those tests used the image 172635_3T_T1w_MPR1.nii and 172635_3T_T2w_SPC1.nii with 1.000 trials on each entropic parameter for Shannon and Tsallis and 2.000 trials for Mattes using the High Quality only for comparison.

For a full log of the trials please check the files: paper_mattes_fixed_T1xT2_MT8.log, paper_tsallis_hcp_t1_t2_translation.log 
Table 21 - Monte Carlo results for translation with same image as fixed and moving, using the NAMIC N2_T1.nrrd image

\begin{tabular}{lccccrrr}
\hline Metric & Q & Binning & $<1.0$ & $<3.0$ & $<5.0$ & Mean & Stddev \\
\hline Mattes & 1.0 & Yes & $63.99 \%$ & $64.09 \%$ & $64.09 \%$ & 61.49 & 82.23 \\
Shannon & 1.0 & No & $89.53 \%$ & $95.21 \%$ & $95.29 \%$ & 7.16 & 29.93 \\
Tsallis & 1.1 & No & $95.16 \%$ & $99.97 \%$ & $99.97 \%$ & 0.79 & 3.45 \\
Tsallis & 1.2 & No & $94.39 \%$ & $99.97 \%$ & $99.97 \%$ & 0.79 & 3.45 \\
Tsallis & 1.3 & No & $95.62 \%$ & $99.97 \%$ & $99.97 \%$ & 0.78 & 3.45 \\
Tsallis & 1.4 & No & $95.77 \%$ & $99.96 \%$ & $99.97 \%$ & 0.80 & 3.45 \\
Tsallis & 1.5 & No & $95.94 \%$ & $99.97 \%$ & $99.97 \%$ & 0.80 & 3.45 \\
Tsallis & 1.6 & No & $97.38 \%$ & $99.95 \%$ & $99.96 \%$ & 0.89 & 3.45 \\
Tsallis & 1.7 & No & $0.26 \%$ & $99.84 \%$ & $99.95 \%$ & 1.87 & 3.43 \\
Tsallis & 1.8 & No & $0.02 \%$ & $3.85 \%$ & $99.82 \%$ & 3.57 & 3.41 \\
Tsallis & 1.9 & No & $0.00 \%$ & $0.17 \%$ & $96.86 \%$ & 4.44 & 3.42 \\
Tsallis & 2.0 & No & $0.00 \%$ & $0.00 \%$ & $3.69 \%$ & 6.28 & 3.65 \\
\hline
\end{tabular}

Source: author.

Table 22 - Monte Carlo results for translation with same image as fixed and moving, using the HCP images with T1 x T2

\begin{tabular}{lccccrrr}
\hline Metric & Q & Binning & $<1.0$ & $<3.0$ & $<5.0$ & Mean & Stddev \\
\hline Mattes & 1.0 & Yes & $58.13 \%$ & $58.23 \%$ & $58.23 \%$ & 55.17 & 72.02 \\
Shannon & 1.0 & No & $99.09 \%$ & $99.09 \%$ & $99.09 \%$ & 1.50 & 8.44 \\
Tsallis & 1.1 & No & $99.09 \%$ & $100.00 \%$ & $100.00 \%$ & 0.62 & 0.10 \\
Tsallis & 1.2 & No & $98.18 \%$ & $100.00 \%$ & $100.00 \%$ & 0.52 & 0.10 \\
Tsallis & 1.3 & No & $99.09 \%$ & $100.00 \%$ & $100.00 \%$ & 0.45 & 0.11 \\
Tsallis & 1.4 & No & $100.00 \%$ & $100.00 \%$ & $100.00 \%$ & 0.53 & 0.08 \\
Tsallis & 1.5 & No & $100.00 \%$ & $100.00 \%$ & $100.00 \%$ & 0.52 & 0.07 \\
Tsallis & 1.6 & No & $98.18 \%$ & $100.00 \%$ & $100.00 \%$ & 0.59 & 0.11 \\
Tsallis & 1.7 & No & $63.30 \%$ & $100.00 \%$ & $100.00 \%$ & 0.97 & 0.09 \\
Tsallis & 1.8 & No & $42.20 \%$ & $100.00 \%$ & $100.00 \%$ & 1.01 & 0.08 \\
Tsallis & 1.9 & No & $22.94 \%$ & $100.00 \%$ & $100.00 \%$ & 1.07 & 0.13 \\
Tsallis & 2.0 & No & $11.93 \%$ & $100.00 \%$ & $100.00 \%$ & 1.08 & 0.10 \\
\hline
\end{tabular}

Source: author.

Table 22 shows the parsed results, with Mattes having a poor performance compared to Shannon and Tsallis. Figure 53 shows the success rate as a function of the loss threshold, i.e., the final position in the registering trial.

\section{B.1.4 Monte Carlo Randomized}


Figure 52 - Monte Carlo results for translation with same image as fixed and moving, using the NAMIC N2_T1.nrrd

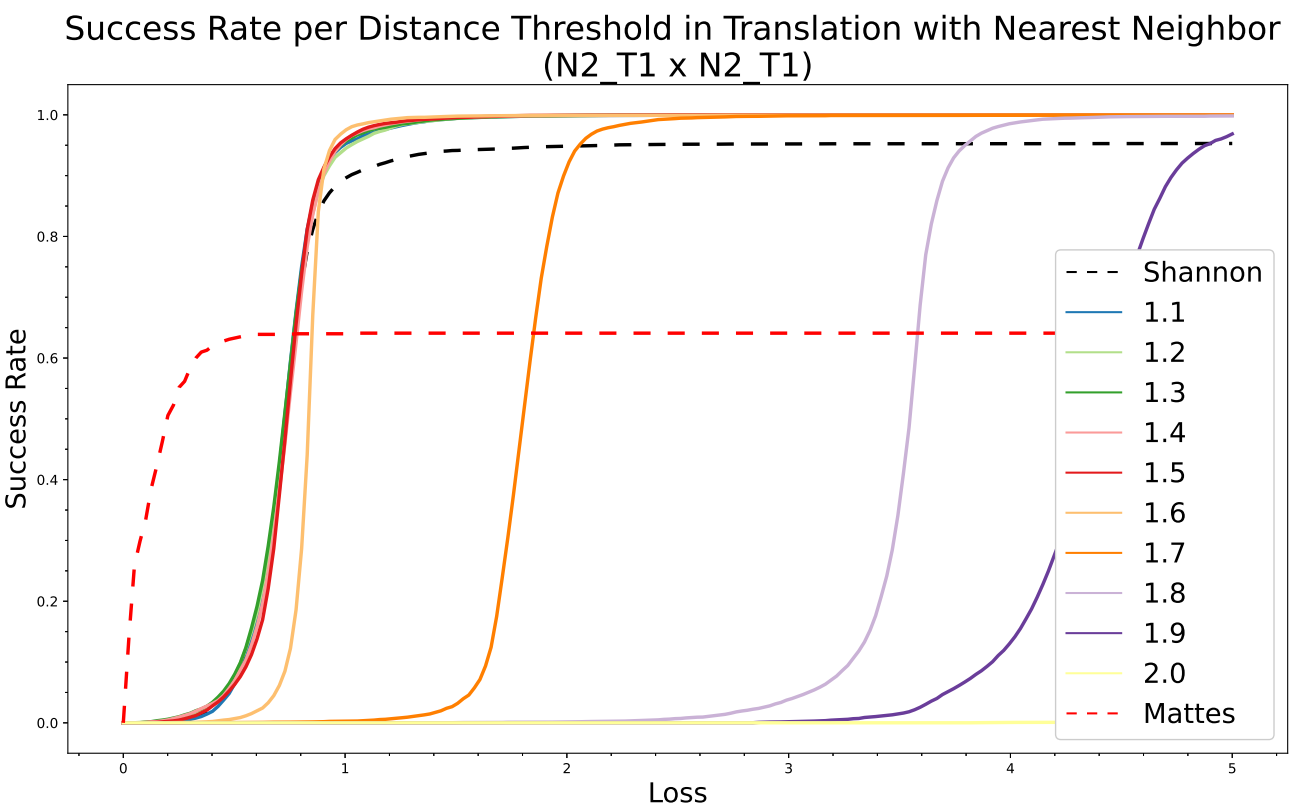

Source: author.

Figure 53 - Monte Carlo results for translation with same image as fixed and moving, using HCP T1 x T2

Success Rate per Distance Threshold in Translation with Nearest Neighbor (HCP T1 x T2)

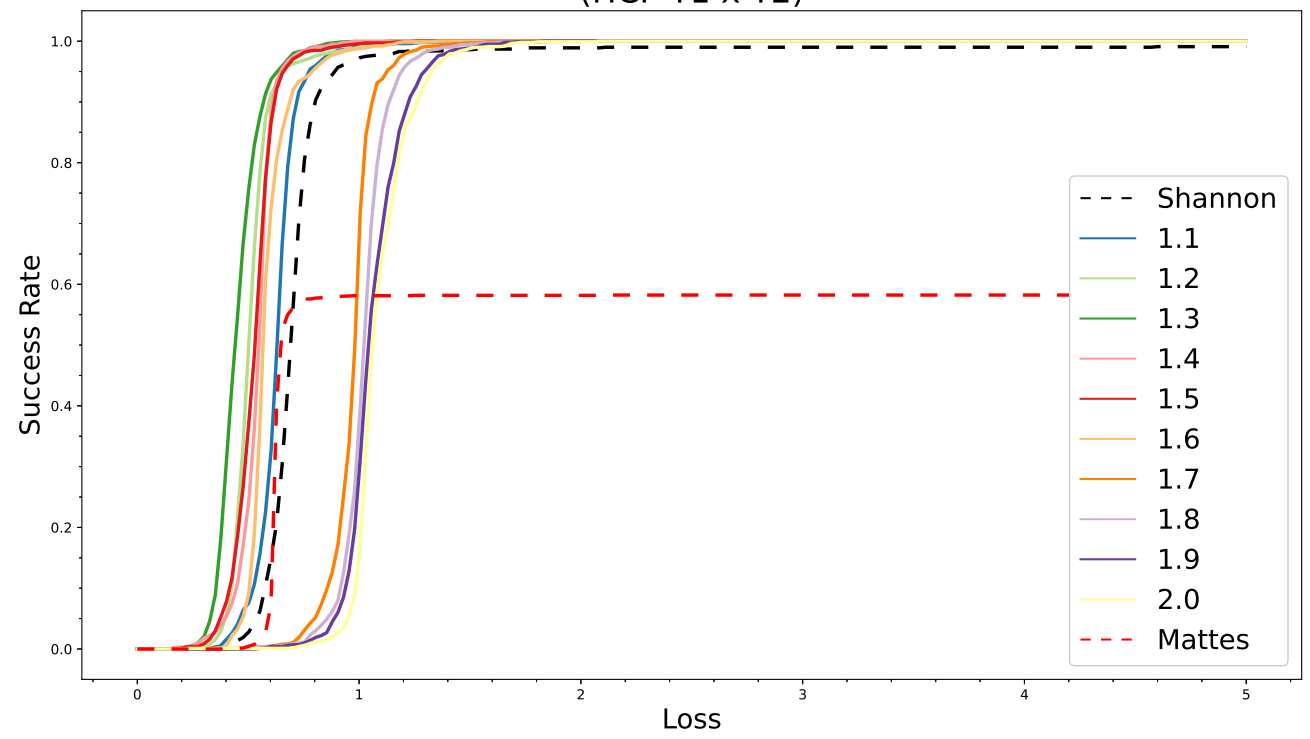

Source: author. 
Figure 54 - Monte Carlo results for translation with same image as fixed and moving, only Mattes

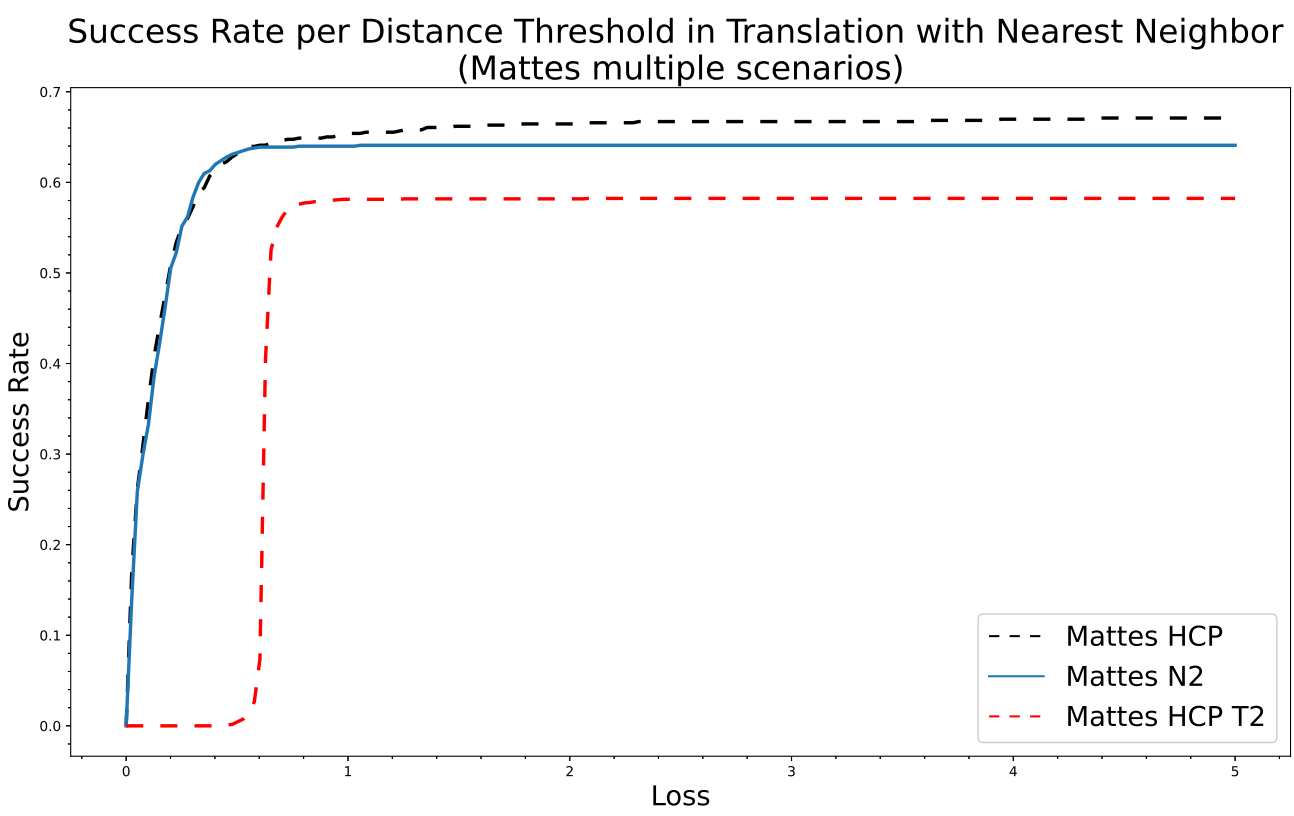

Source: author.

Figure 55 - Monte Carlo results for translation with same image as fixed and moving, only Shannon

Success Rate per Distance Threshold in Translation with Nearest Neighbor (Shannon multiple scenarios)

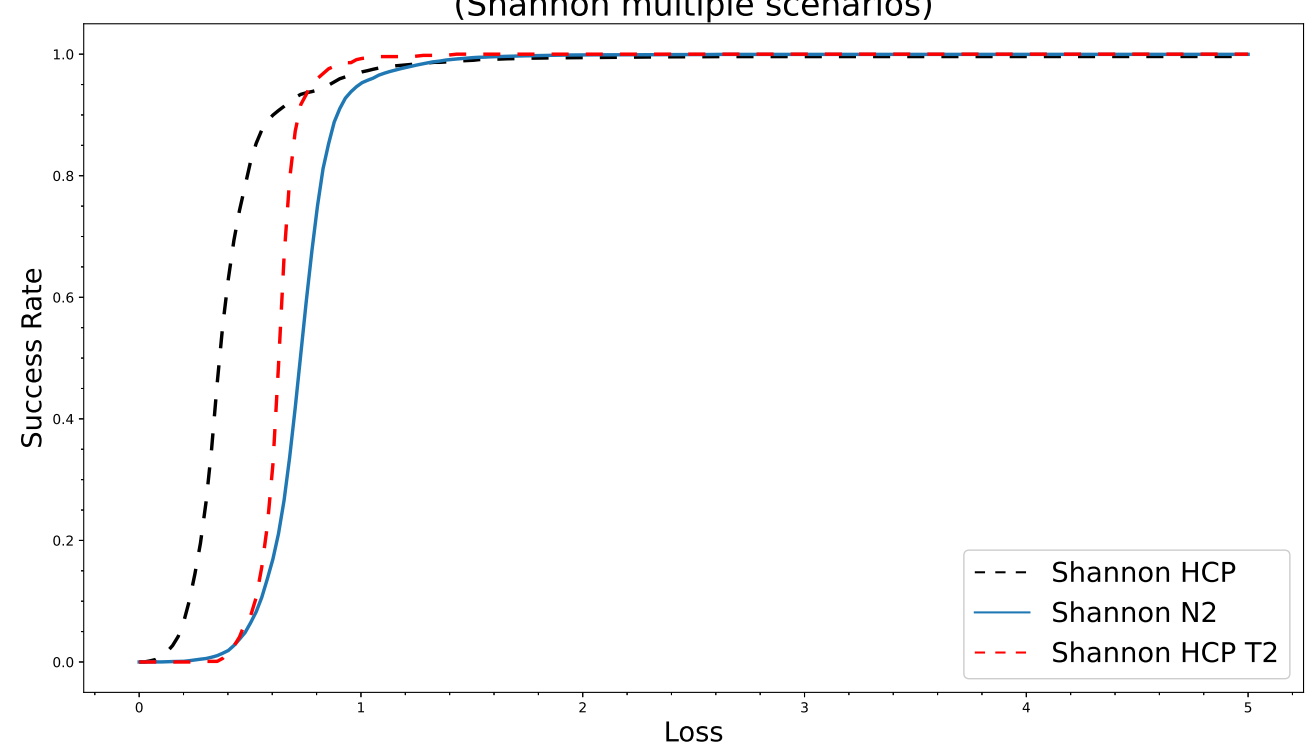

Source: author. 
Figure 56 - Monte Carlo results for translation with same image as fixed and moving, only Tsallis

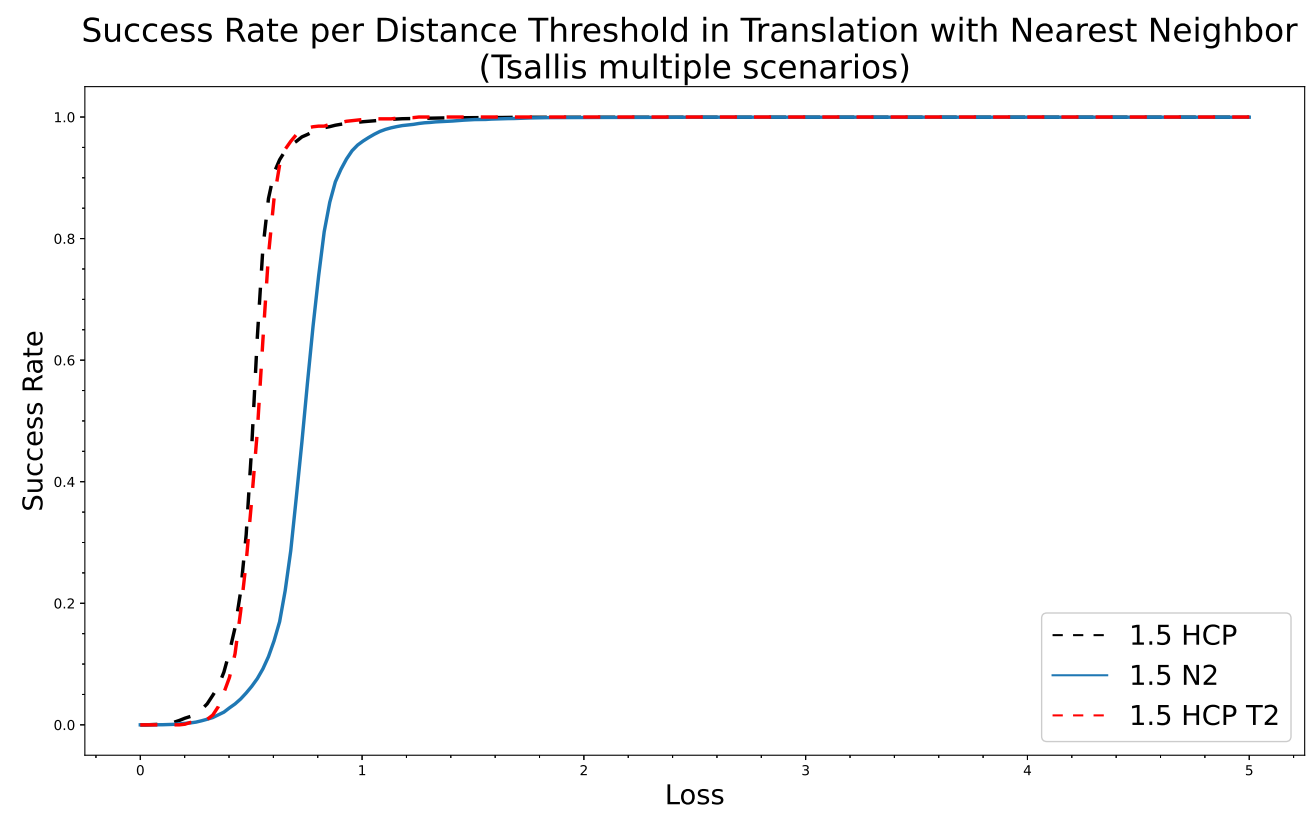

Source: author. 
Figure 57 - Monte Carlo results stripplot for translation with different methods on the lines and the column loss is the final distance for the registration trials, log scale on y-axis

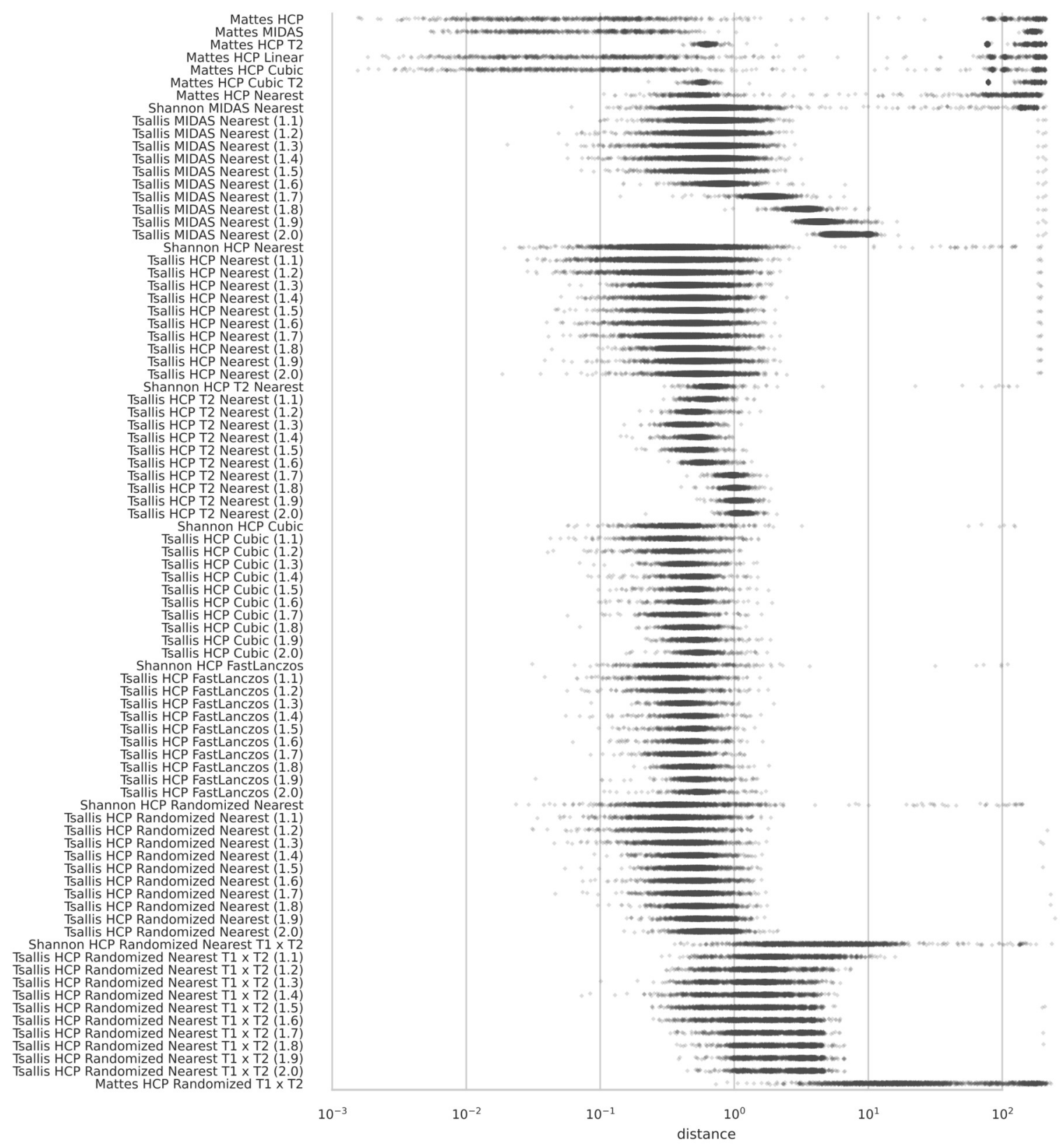

Source: author. 
Figure 58 - Monte Carlo results stripplot for translation with different methods on the lines and the column loss is the final distance for the registration trials, linear scale on y-axis
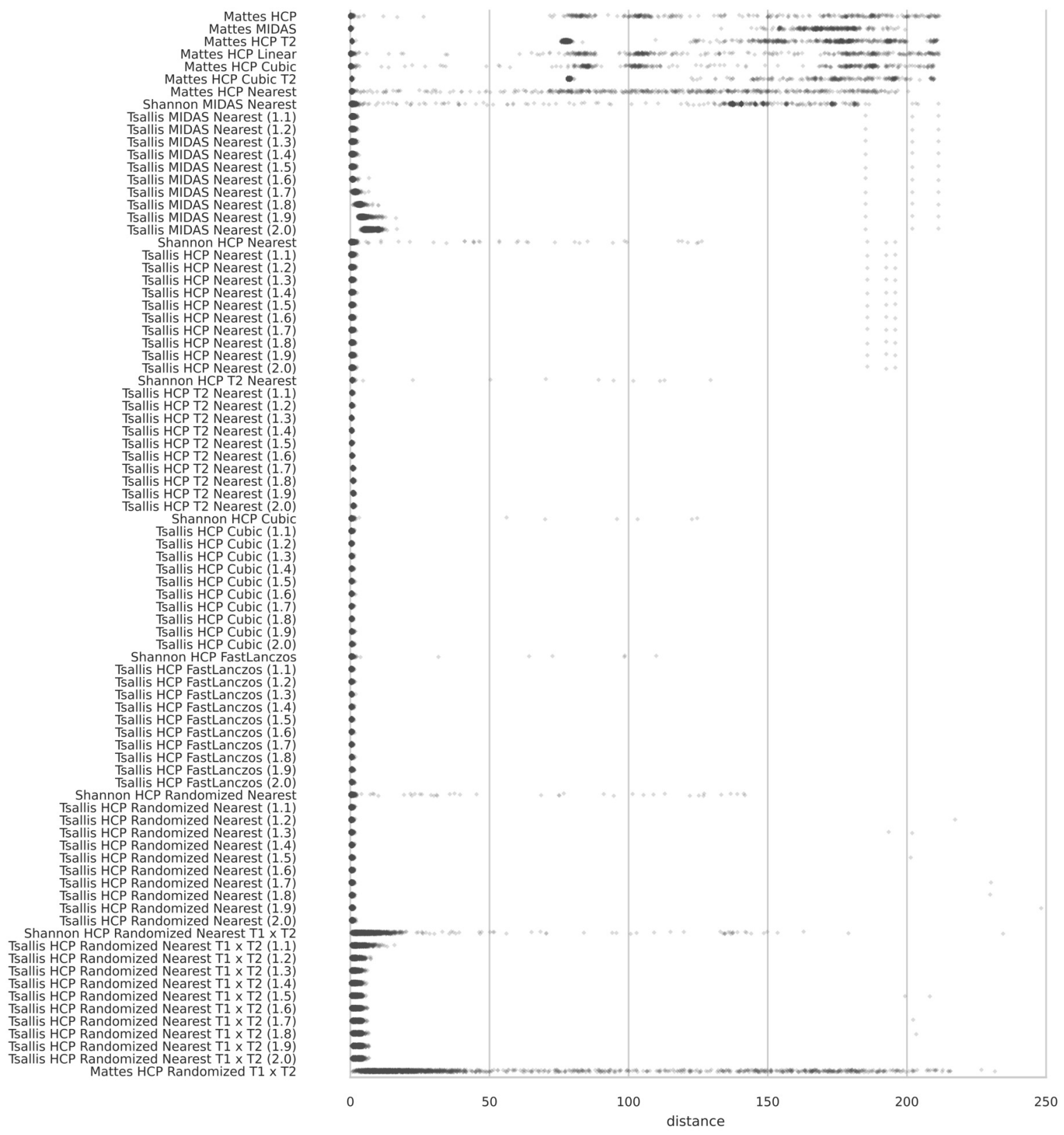

Source: author. 
Figure 59 - Monte Carlo results stripplot combined with statistical point plot for translation with different methods on the lines and the column loss is the final distance for the registration trials, log scale on y-axis

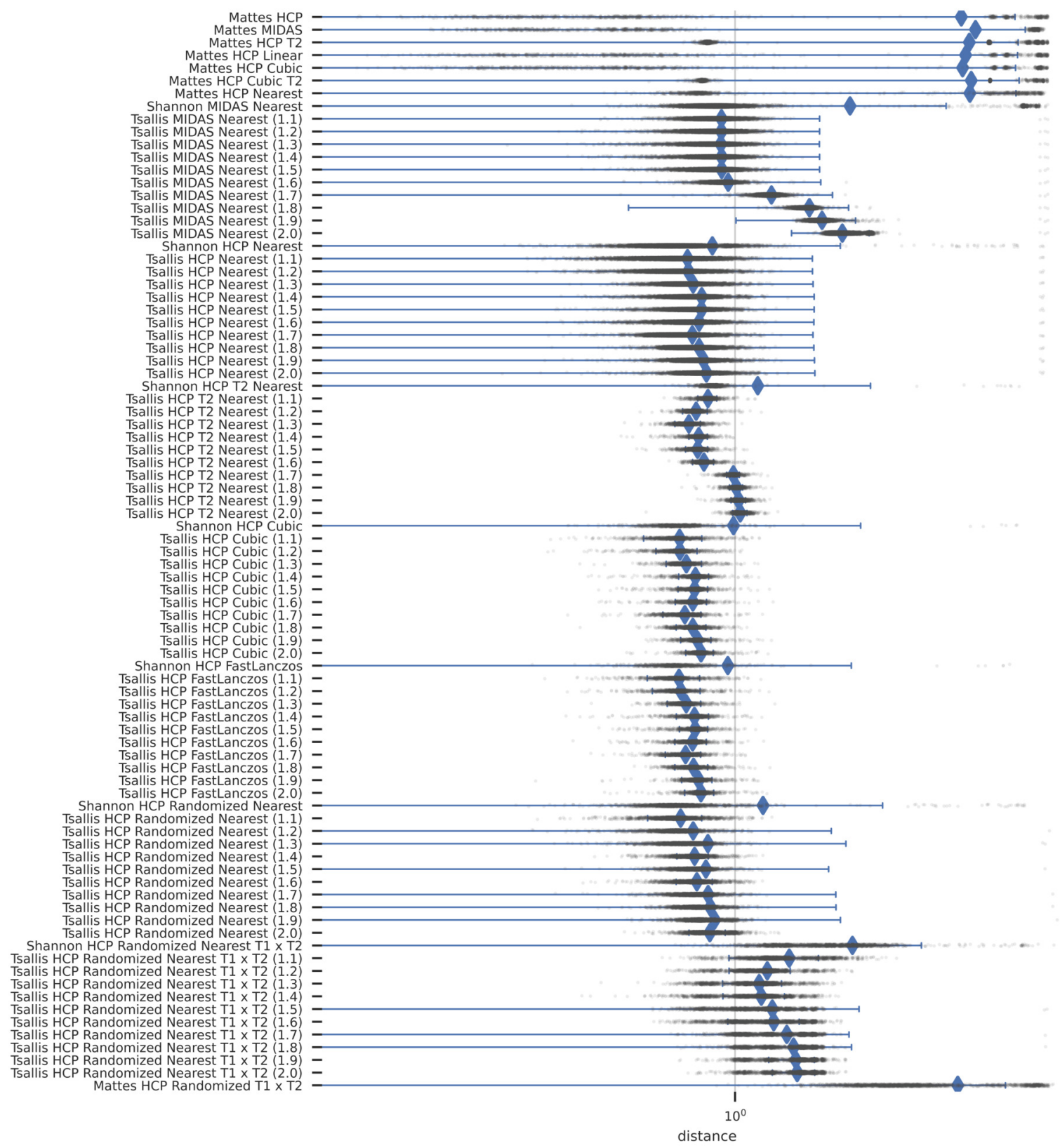

Source: author. 
Figure 60 - Monte Carlo results stripplot combined with statistical point plot for translation with different methods on the lines and the column loss is the final distance for the registration trials, linear scale on y-axis
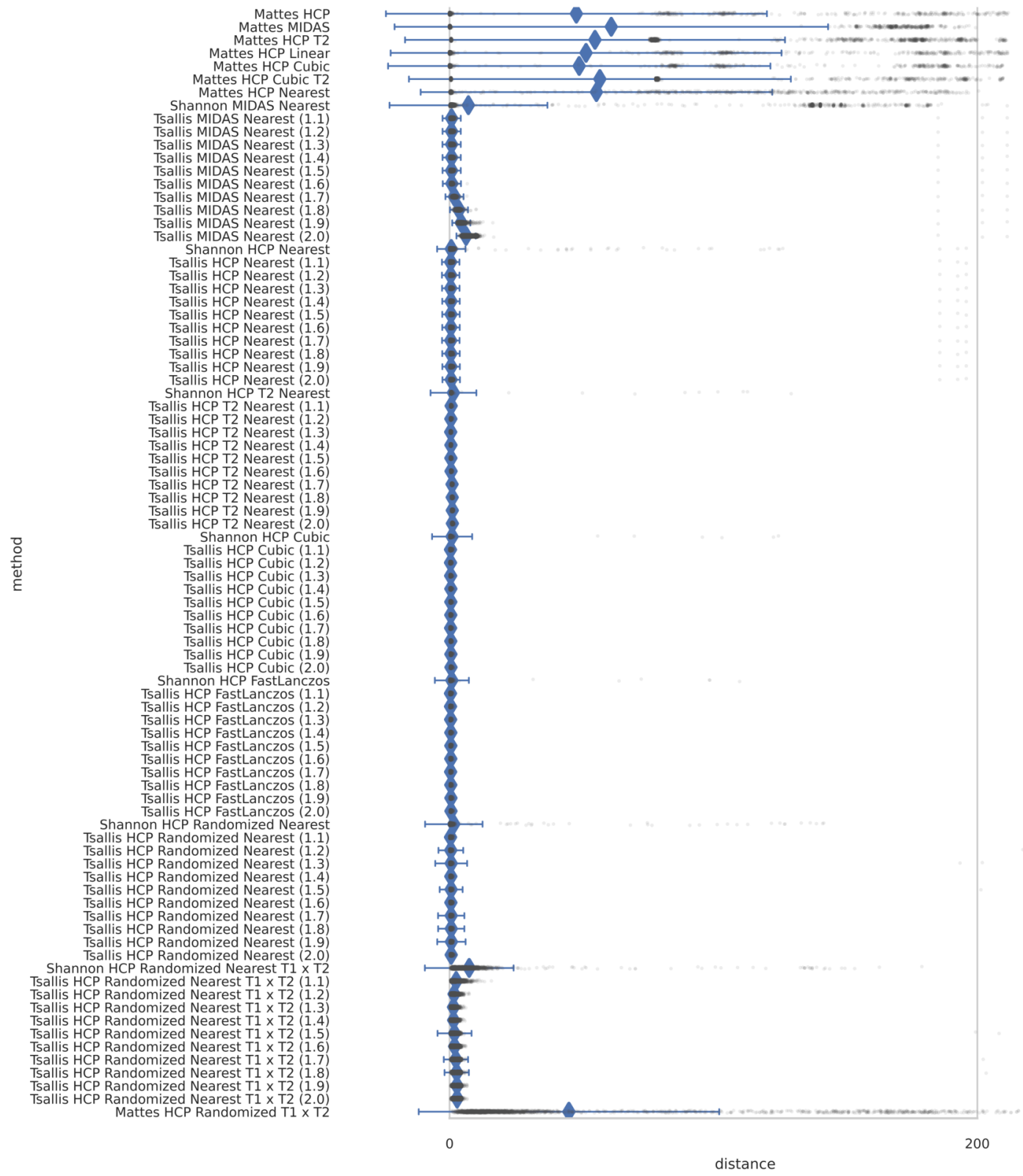

Source: author. 
Table 23 - All Monte Carlo results collected

\begin{tabular}{|c|c|c|c|c|c|}
\hline \multirow[b]{2}{*}{ Method } & \multirow[b]{2}{*}{ Mean } & \multirow[b]{2}{*}{ Deviation } & \multicolumn{3}{|c|}{ Acceptable distance } \\
\hline & & & $1 \mathrm{~mm}$ & $3 \mathrm{~mm}$ & $5 \mathrm{~mm}$ \\
\hline Mattes HCP & 48.0785 & 72.2392 & $64.7000 \%$ & $65.8000 \%$ & $66.0000 \%$ \\
\hline Mattes MIDAS & 61.3010 & 82.1740 & $64.1000 \%$ & $64.2000 \%$ & $64.2000 \%$ \\
\hline Mattes HCP T2 & 55.1406 & 72.0132 & $58.1500 \%$ & $58.2500 \%$ & $58.2500 \%$ \\
\hline Mattes HCP Linear & 51.7435 & 74.1020 & $61.8000 \%$ & $63.2000 \%$ & $63.4000 \%$ \\
\hline Mattes HCP Cubic & 49.1481 & 72.4739 & $63.3000 \%$ & $64.4000 \%$ & $64.5000 \%$ \\
\hline Mattes HCP Cubic T2 & 56.9713 & 72.3993 & $56.2000 \%$ & $56.3000 \%$ & $56.3000 \%$ \\
\hline Mattes HCP Nearest & 55.6754 & 66.6591 & $51.5000 \%$ & $53.3000 \%$ & $53.7000 \%$ \\
\hline Shannon MIDAS Nearest & 7.1605 & 29.9228 & $89.5200 \%$ & $95.2100 \%$ & $95.2900 \%$ \\
\hline Tsallis MIDAS Nearest (1.1) & 0.7935 & 3.4519 & $95.1600 \%$ & $99.9700 \%$ & $99.9700 \%$ \\
\hline Tsallis MIDAS Nearest (1.2) & 0.7922 & 3.4524 & $94.3900 \%$ & $99.9700 \%$ & $99.9700 \%$ \\
\hline Tsallis MIDAS Nearest (1.3) & 0.7847 & 3.4524 & $95.6200 \%$ & $99.9700 \%$ & $99.9700 \%$ \\
\hline Tsallis MIDAS Nearest (1.4) & 0.7965 & 3.4517 & $95.7700 \%$ & $99.9600 \%$ & $99.9700 \%$ \\
\hline Tsallis MIDAS Nearest (1.5) & 0.7964 & 3.4512 & $95.9400 \%$ & $99.9700 \%$ & $99.9700 \%$ \\
\hline Tsallis MIDAS Nearest (1.6) & 0.8901 & 3.4478 & $97.3800 \%$ & $99.9500 \%$ & $99.9600 \%$ \\
\hline Tsallis MIDAS Nearest (1.7) & 1.8666 & 3.4343 & $0.2600 \%$ & $99.8400 \%$ & $99.9500 \%$ \\
\hline Tsallis MIDAS Nearest (1.8) & 3.5719 & 3.4105 & $0.0200 \%$ & $3.8500 \%$ & $99.8200 \%$ \\
\hline Tsallis MIDAS Nearest (1.9) & 4.4371 & 3.4211 & $0.0000 \%$ & $0.1700 \%$ & $96.8600 \%$ \\
\hline Tsallis MIDAS Nearest (2.0) & 6.2781 & 3.6497 & $0.0000 \%$ & $0.0000 \%$ & $3.6900 \%$ \\
\hline Shannon HCP Nearest & 0.6790 & 5.3831 & $97.0400 \%$ & $99.6100 \%$ & $99.6200 \%$ \\
\hline Tsallis HCP Nearest (1.1) & 0.4438 & 3.3134 & $98.8300 \%$ & $99.9700 \%$ & $99.9700 \%$ \\
\hline
\end{tabular}




\begin{tabular}{llllll} 
Method & Mean & Deviation & $1 \mathrm{~mm}$ & $3 \mathrm{~mm}$ & $5 \mathrm{~mm}$ \\
\hline Shannon HCP Cubic & 0.9742 & 7.6044 & $97.0000 \%$ & $99.3000 \%$ & $99.4000 \%$ \\
Tsallis HCP Cubic (1.1) & 0.3876 & 0.1783 & $98.3000 \%$ & $100.0000 \%$ & $100.0000 \%$ \\
Tsallis HCP Cubic (1.2) & 0.3898 & 0.1318 & $99.7000 \%$ & $100.0000 \%$ & $100.0000 \%$ \\
Tsallis HCP Cubic (1.3) & 0.4353 & 0.1275 & $99.2000 \%$ & $100.0000 \%$ & $100.0000 \%$ \\
Tsallis HCP Cubic (1.4) & 0.5082 & 0.1274 & $99.1000 \%$ & $100.0000 \%$ & $100.0000 \%$ \\
Tsallis HCP Cubic (1.5) & 0.5013 & 0.1243 & $99.4000 \%$ & $100.0000 \%$ & $100.0000 \%$ \\
Tsallis HCP Cubic (1.6) & 0.4865 & 0.1269 & $99.4000 \%$ & $100.0000 \%$ & $100.0000 \%$ \\
Tsallis HCP Cubic (1.7) & 0.4238 & 0.1333 & $99.1000 \%$ & $100.0000 \%$ & $100.0000 \%$ \\
Tsallis HCP Cubic (1.8) & 0.4861 & 0.1212 & $99.6000 \%$ & $100.0000 \%$ & $100.0000 \%$ \\
Tsallis HCP Cubic (1.9) & 0.5274 & 0.1334 & $98.5000 \%$ & $100.0000 \%$ & $100.0000 \%$ \\
Tsallis HCP Cubic (2.0) & 0.5592 & 0.1290 & $98.5000 \%$ & $100.0000 \%$ & $100.0000 \%$ \\
Shannon HCP FastLanczos & 0.8844 & 6.4355 & $96.6000 \%$ & $99.3000 \%$ & $99.4000 \%$ \\
Tsallis HCP FastLanczos (1.1) & 0.3836 & 0.1614 & $98.8000 \%$ & $100.0000 \%$ & $100.0000 \%$ \\
Tsallis HCP FastLanczos (1.2) & 0.3962 & 0.1536 & $98.9000 \%$ & $100.0000 \%$ & $100.0000 \%$ \\
Tsallis HCP FastLanczos (1.3) & 0.4343 & 0.1208 & $99.6000 \%$ & $100.0000 \%$ & $100.0000 \%$ \\
Tsallis HCP FastLanczos (1.4) & 0.5000 & 0.1339 & $98.9000 \%$ & $100.0000 \%$ & $100.0000 \%$ \\
Tsallis HCP FastLanczos (1.5) & 0.5057 & 0.1194 & $99.4000 \%$ & $100.0000 \%$ & $100.0000 \%$ \\
Tsallis HCP FastLanczos (1.6) & 0.4844 & 0.1275 & $99.5000 \%$ & $100.0000 \%$ & $100.0000 \%$ \\
Tsallis HCP FastLanczos (1.7) & 0.4291 & 0.1258 & $99.3000 \%$ & $100.0000 \%$ & $100.0000 \%$ \\
Tsallis HCP FastLanczos (1.8) & 0.4919 & 0.1351 & $99.1000 \%$ & $100.0000 \%$ & $100.0000 \%$ \\
\hline
\end{tabular}




\begin{tabular}{llllll} 
Method & Mean & Deviation & $1 \mathrm{~mm}$ & $3 \mathrm{~mm}$ & $5 \mathrm{~mm}$ \\
\hline Tsallis HCP Randomized Nearest T1 x T2 (1.7) & 2.4258 & 4.6032 & $9.9000 \%$ & $61.4500 \%$ & $99.7000 \%$ \\
Tsallis HCP Randomized Nearest T1 x T2 (1.8) & 2.7242 & 4.6128 & $4.7500 \%$ & $49.9500 \%$ & $99.4000 \%$ \\
Tsallis HCP Randomized Nearest T1 x T2 (1.9) & 2.8284 & 1.0490 & $3.1000 \%$ & $42.9000 \%$ & $99.2500 \%$ \\
Tsallis HCP Randomized Nearest T1 x T2 (2.0) & 2.8983 & 1.0050 & $2.4500 \%$ & $40.8500 \%$ & $99.3000 \%$ \\
Mattes HCP Randomized T1 x T2 & 45.2538 & 56.9377 & $0.1000 \%$ & $1.1000 \%$ & $5.0500 \%$ \\
\hline
\end{tabular}

Source: author. 